\title{
Theoretical Studies of Allowed (E1) and Forbidden (E2 and M1) Transitions in La IV Supplement
}

\author{
B. KARAÇOBAN Usta ${ }^{a, *}$ AND S. EsER ${ }^{b}$ \\ ${ }^{a}$ Department of Fundamental Science in Engineering, Sakarya University of Applied Science, 54187, Sakarya, Turkey \\ ${ }^{b}$ Department of Physics, Sakarya University, 54187, Sakarya, Turkey
}

DOI: 10.12693/APhysPolA.137.1178.S1

TABLE I

New data (energies $E$, Landé $g$-factors, and lifetimes $\tau$ ) for valence excitation states in La IV.

\begin{tabular}{c|c|c|c|c|c|c}
\hline \hline \multirow{2}{*}{ Conf. } & \multicolumn{3}{|c|}{ Term } & \multirow{2}{*}{$\mathrm{J}\left[\mathrm{cm}^{-1}\right]$} & $g$-factor & $\tau[\mathrm{ns}]$ \\
\cline { 2 - 6 } & $j K$ & $L S$ & & HFR & HFR & HFR \\
\hline $5 p^{5} 6 f$ & $3 / 2[3 / 2]$ & ${ }^{3} D$ & 1 & 333647.94 & 0.504 & 0.304 \\
& $3 / 2[9 / 2]$ & ${ }^{3} G$ & 5 & 333926.39 & 1.200 & 0.306 \\
& $3 / 2[3 / 2]$ & ${ }^{3} D$ & 2 & 333953.73 & 1.110 & 0.329 \\
& $3 / 2[9 / 2]$ & ${ }^{1} G$ & 4 & 333997.47 & 1.026 & 0.311 \\
& $3 / 2[5 / 2]$ & ${ }^{3} D$ & 3 & 334439.33 & 1.189 & 0.311 \\
& $3 / 2[5 / 2]$ & ${ }^{1} D$ & 2 & 334707.81 & 0.850 & 0.376 \\
& $3 / 2[7 / 2]$ & ${ }^{1} F$ & 3 & 334807.01 & 0.955 & 0.309 \\
& $3 / 2[7 / 2]$ & ${ }^{3} F$ & 4 & 334912.85 & 1.186 & 0.322 \\
& $1 / 2[7 / 2]$ & ${ }^{3} G$ & 3 & 355019.15 & 0.883 & 0.360 \\
& $1 / 2[7 / 2]$ & ${ }^{3} G$ & 4 & 355824.58 & 1.140 & 0.386 \\
& $1 / 2[5 / 2]$ & ${ }^{3} F$ & 2 & 355876.54 & 0.879 & 0.356 \\
& $1 / 2[5 / 2]$ & ${ }^{3} F$ & 3 & 355894.90 & 1.189 & 0.372 \\
\hline $5 p^{5} 7 f$ & $3 / 2[3 / 2]$ & ${ }^{3} D$ & 1 & 354652.00 & 0.501 & 0.496 \\
& $3 / 2[3 / 2]$ & ${ }^{3} D$ & 2 & 354696.26 & 1.060 & 0.457 \\
& $3 / 2[5 / 2]$ & ${ }^{3} D$ & 3 & 354778.24 & 1.189 & 0.399 \\
& $3 / 2[9 / 2]$ & ${ }^{3} G$ & 5 & 354950.64 & 1.200 & 0.509 \\
& $3 / 2[9 / 2]$ & ${ }^{3} G$ & 4 & 354953.62 & 1.048 & 0.470 \\
& $3 / 2[7 / 2]$ & ${ }^{3} F$ & 4 & 355225.44 & 1.115 & 0.433 \\
& $3 / 2[5 / 2]$ & ${ }^{1} D$ & 2 & 355303.10 & 0.891 & 0.674 \\
& $3 / 2[7 / 2]$ & ${ }^{1} F$ & 3 & 355754.41 & 0.904 & 0.389 \\
& $1 / 2[7 / 2]$ & ${ }^{3} G$ & 3 & 376386.33 & 0.856 & 0.542 \\
& $1 / 2[7 / 2]$ & ${ }^{1} G$ & 4 & 376457.97 & 1.102 & 0.547 \\
& $1 / 2[5 / 2]$ & ${ }^{3} F$ & 3 & 376496.66 & 1.197 & 0.545 \\
& $1 / 2[5 / 2]$ & ${ }^{3} F$ & 2 & 376544.92 & 0.888 & 0.558 \\
\hline \multirow{6nnnnn}{*}{$3 / 2[3 / 2]$} & ${ }^{3} D$ & 1 & 367479.26 & 0.500 & 0.806 \\
& $3 / 2[3 / 2]$ & ${ }^{3} D$ & 2 & 367586.52 & 1.102 & 0.828 \\
& $3 / 2[9 / 2]$ & ${ }^{3} G$ & 5 & 367619.03 & 1.200 & 0.812 \\
& $3 / 2[9 / 2]$ & ${ }^{1} G$ & 4 & 367648.89 & 1.027 & 0.799 \\
& $3 / 2[5 / 2]$ & ${ }^{3} F$ & 3 & 367788.35 & 1.182 & 0.784 \\
& $3 / 2[5 / 2]$ & ${ }^{1} D$ & 2 & 367913.80 & 0.848 & 0.869 \\
& & & & &
\end{tabular}

*corresponding author; e-mail: bkaracoban@subu.edu.tr
TABLE I (cont.)

\begin{tabular}{|c|c|c|c|c|c|c|}
\hline \multirow{2}{*}{ Conf. } & \multicolumn{2}{|l|}{ Term } & \multirow{2}{*}{$\mathrm{J}$} & \multirow{2}{*}{$\begin{array}{c}E\left[\mathrm{~cm}^{-1}\right] \\
\mathrm{HFR} \\
\end{array}$} & \multirow{2}{*}{$\begin{array}{c}g \text {-factor } \\
\text { HFR }\end{array}$} & \multirow{2}{*}{$\begin{array}{c}\tau[\mathrm{ns}] \\
\mathrm{HFR}\end{array}$} \\
\hline & $j K$ & $L S$ & & & & \\
\hline \multirow[t]{6}{*}{$5 p^{5} 8 f$} & $3 / 2[7 / 2]$ & ${ }^{1} F$ & 3 & 367914.23 & 0.959 & 0.753 \\
\hline & $3 / 2[7 / 2]$ & ${ }^{3} F$ & 4 & 367956.03 & 1.188 & 0.772 \\
\hline & $1 / 2[7 / 2]$ & ${ }^{3} G$ & 3 & 388925.13 & 0.824 & 0.770 \\
\hline & $1 / 2[5 / 2]$ & ${ }^{3} D$ & 3 & 388973.88 & 1.203 & 0.786 \\
\hline & $1 / 2[7 / 2]$ & ${ }^{3} G$ & 4 & 388989.60 & 1.085 & 0.794 \\
\hline & $1 / 2[5 / 2]$ & ${ }^{3} F$ & 2 & 389052.10 & 0.885 & 0.812 \\
\hline \multirow[t]{12}{*}{$5 p^{5} 9 f$} & $3 / 2[3 / 2]$ & ${ }^{3} D$ & 1 & 375746.84 & 0.500 & 1.371 \\
\hline & $3 / 2[3 / 2]$ & ${ }^{3} D$ & 2 & 375780.68 & 1.079 & 1.216 \\
\hline & $3 / 2[5 / 2]$ & ${ }^{3} F$ & 3 & 375851.23 & 1.184 & 1.015 \\
\hline & $3 / 2[9 / 2]$ & ${ }^{3} G$ & 5 & 375857.26 & 1.200 & 1.316 \\
\hline & $3 / 2[9 / 2]$ & ${ }^{3} G$ & 4 & 375867.11 & 1.037 & 1.173 \\
\hline & $3 / 2[7 / 2]$ & ${ }^{1} F$ & 3 & 375956.45 & 0.930 & 0.893 \\
\hline & $3 / 2[7 / 2]$ & ${ }^{3} F$ & 4 & 376024.45 & 1.162 & 0.982 \\
\hline & $3 / 2[5 / 2]$ & ${ }^{1} D$ & 2 & 376042.81 & 0.862 & 0.971 \\
\hline & $1 / 2[7 / 2]$ & ${ }^{3} G$ & 3 & 397142.04 & 0.823 & 1.196 \\
\hline & $1 / 2[5 / 2]$ & ${ }^{3} D$ & 3 & 397171.26 & 1.204 & 1.205 \\
\hline & $1 / 2[7 / 2]$ & ${ }^{1} G$ & 4 & 397185.88 & 1.084 & 1.170 \\
\hline & $1 / 2[5 / 2]$ & ${ }^{3} F$ & 2 & 397228.72 & 0.886 & 1.023 \\
\hline \multirow[t]{12}{*}{$5 p^{5} 10 f$} & $3 / 2[3 / 2]$ & ${ }^{3} D$ & 1 & 381470.85 & 0.503 & 1.086 \\
\hline & $3 / 2[9 / 2]$ & ${ }^{3} G$ & 5 & 381511.42 & 1.200 & 1.283 \\
\hline & $3 / 2[9 / 2]$ & ${ }^{1} G$ & 4 & 381525.67 & 1.027 & 1.596 \\
\hline & $3 / 2[3 / 2]$ & ${ }^{3} D$ & 2 & 381526.45 & 1.093 & 1.225 \\
\hline & $3 / 2[5 / 2]$ & ${ }^{3} F$ & 3 & 381596.36 & 1.179 & 1.298 \\
\hline & $3 / 2[7 / 2]$ & ${ }^{1} F$ & 3 & 381650.61 & 0.960 & 1.436 \\
\hline & $3 / 2[5 / 2]$ & ${ }^{1} D$ & 2 & 381659.81 & 0.856 & 2.396 \\
\hline & $3 / 2[7 / 2]$ & ${ }^{3} F$ & 4 & 381670.45 & 1.189 & 1.520 \\
\hline & $1 / 2[7 / 2]$ & ${ }^{3} G$ & 3 & 402785.08 & 0.823 & 1.299 \\
\hline & $1 / 2[5 / 2]$ & ${ }^{3} D$ & 3 & 402804.63 & 1.204 & 1.243 \\
\hline & $1 / 2[7 / 2]$ & ${ }^{3} G$ & 4 & 402816.52 & 1.084 & 1.533 \\
\hline & $1 / 2[5 / 2]$ & ${ }^{3} F$ & 2 & 402847.18 & 0.887 & 1.612 \\
\hline \multirow[t]{6}{*}{$5 p^{5} 8 p$} & $3 / 2[1 / 2]$ & ${ }^{3} S$ & 1 & 340268.59 & 1.779 & 1.970 \\
\hline & $3 / 2[5 / 2]$ & ${ }^{1} D$ & 2 & 340572.87 & 1.134 & 2.206 \\
\hline & $3 / 2[5 / 2]$ & ${ }^{3} D$ & 3 & 341191.84 & 1.333 & 1.991 \\
\hline & $3 / 2[3 / 2]$ & ${ }^{1} P$ & 1 & 341472.31 & 1.071 & 2.434 \\
\hline & $3 / 2[3 / 2]$ & ${ }^{3} P$ & 2 & 341776.29 & 1.364 & 2.132 \\
\hline & $3 / 2[1 / 2]$ & ${ }^{1} S$ & 0 & 342829.00 & 0.000 & 3.584 \\
\hline
\end{tabular}


TABLE I (cont.)

TABLE I (cont.)

\begin{tabular}{|c|c|c|c|c|c|c|}
\hline \multirow{2}{*}{ Conf. } & \multicolumn{2}{|l|}{ Term } & \multirow{2}{*}{ J } & \multirow{2}{*}{$\begin{array}{c}E\left[\mathrm{~cm}^{-1}\right] \\
\mathrm{HFR}\end{array}$} & \multirow{2}{*}{$\frac{g \text {-factor }}{\text { HFR }}$} & \multirow{2}{*}{$\begin{array}{c}\tau[\mathrm{ns}] \\
\mathrm{HFR} \\
\end{array}$} \\
\hline & $j K$ & $L S$ & & & & \\
\hline \multirow[t]{4}{*}{$5 p^{5} 8 p$} & $1 / 2[3 / 2]$ & ${ }^{3} D$ & 1 & 361736.76 & 0.655 & 2.238 \\
\hline & $1 / 2[1 / 2]$ & ${ }^{3} P$ & 1 & 362627.96 & 1.504 & 2.081 \\
\hline & $1 / 2[3 / 2]$ & ${ }^{3} D$ & 2 & 362656.76 & 1.169 & 2.123 \\
\hline & $1 / 2[1 / 2]$ & ${ }^{3} P$ & 0 & 362821.14 & 0.000 & 3.111 \\
\hline \multirow{10}{*}{$5 p^{5} 9 p$} & $3 / 2[1 / 2]$ & ${ }^{3} S$ & 1 & 358676.75 & 1.777 & 2.899 \\
\hline & $3 / 2[5 / 2]$ & ${ }^{1} D$ & 2 & 358934.14 & 1.137 & 3.380 \\
\hline & $3 / 2[5 / 2]$ & ${ }^{3} D$ & 3 & 359325.20 & 1.333 & 3.148 \\
\hline & $3 / 2[3 / 2]$ & ${ }^{1} P$ & 1 & 359431.95 & 1.057 & 3.759 \\
\hline & $3 / 2[3 / 2]$ & ${ }^{3} \mathrm{P}$ & 2 & 359634.84 & 1.361 & 3.521 \\
\hline & $3 / 2[1 / 2]$ & ${ }^{1} S$ & 0 & 360128.63 & 0.000 & 4.781 \\
\hline & $1 / 2[3 / 2]$ & ${ }^{3} D$ & 1 & 380111.00 & 0.654 & 3.461 \\
\hline & $1 / 2[1 / 2]$ & ${ }^{3} P$ & 1 & 380632.84 & 1.500 & 3.282 \\
\hline & $1 / 2[3 / 2]$ & ${ }^{3} D$ & 2 & 380654.02 & 1.166 & 3.370 \\
\hline & $1 / 2[1 / 2]$ & ${ }^{3} P$ & 0 & 380721.84 & 0.000 & 4.715 \\
\hline \multirow[t]{10}{*}{$5 p^{5} 10 p$} & $3 / 2[1 / 2]$ & ${ }^{3} S$ & 1 & 370087.94 & 1.763 & 3.657 \\
\hline & $3 / 2[5 / 2]$ & ${ }^{1} D$ & 2 & 370191.87 & 1.135 & 4.849 \\
\hline & $3 / 2[5 / 2]$ & ${ }^{3} D$ & 3 & 370436.93 & 1.333 & 4.213 \\
\hline & $3 / 2[3 / 2]$ & ${ }^{1} P$ & 1 & 370532.41 & 1.076 & 5.404 \\
\hline & $3 / 2[3 / 2]$ & ${ }^{3} P$ & 2 & 370645.33 & 1.364 & 5.638 \\
\hline & $3 / 2[1 / 2]$ & ${ }^{1} S$ & 0 & 371081.12 & 0.000 & 9.602 \\
\hline & $1 / 2[3 / 2]$ & ${ }^{3} D$ & 1 & 391404.92 & 0.664 & 4.448 \\
\hline & $1 / 2[1 / 2]$ & ${ }^{3} P$ & 1 & 391729.70 & 1.497 & 4.282 \\
\hline & $1 / 2[3 / 2]$ & ${ }^{3} D$ & 2 & 391764.01 & 1.168 & 4.699 \\
\hline & $1 / 2[1 / 2]$ & ${ }^{3} \mathrm{P}$ & 0 & 391789.67 & 0.000 & 6.398 \\
\hline \multirow[t]{12}{*}{$5 p^{5} 6 h$} & $3 / 2[7 / 2]$ & ${ }^{3} G$ & 3 & 353844.42 & 0.750 & 2.093 \\
\hline & $3 / 2[7 / 2]$ & ${ }^{3} G$ & 4 & 353846.90 & 1.028 & 2.106 \\
\hline & $3 / 2[13 / 2]$ & ${ }^{1} I$ & 6 & 353908.88 & 1.011 & 2.200 \\
\hline & $3 / 2[13 / 2]$ & ${ }^{3} I$ & 7 & 353909.80 & 1.143 & 2.196 \\
\hline & $3 / 2[9 / 2]$ & ${ }^{3} H$ & 4 & 354072.15 & 0.890 & 2.254 \\
\hline & $3 / 2[9 / 2]$ & ${ }^{3} G$ & 5 & 354073.10 & 1.093 & 2.253 \\
\hline & $3 / 2[11 / 2]$ & ${ }^{1} H$ & 5 & 354133.97 & 0.963 & 2.274 \\
\hline & $3 / 2[11 / 2]$ & ${ }^{3} H$ & 6 & 354135.70 & 1.123 & 2.276 \\
\hline & $1 / 2[9 / 2]$ & ${ }^{1} G$ & 4 & 375227.66 & 0.926 & 2.375 \\
\hline & $1 / 2[9 / 2]$ & ${ }^{3} H$ & 5 & 375227.75 & 1.122 & 2.372 \\
\hline & $1 / 2[11 / 2]$ & ${ }^{3} I$ & 5 & 375235.63 & 0.890 & 2.297 \\
\hline & $1 / 2[11 / 2]$ & ${ }^{3} I$ & 6 & 375237.75 & 1.061 & 2.302 \\
\hline \multirow[t]{12}{*}{$5 p^{5} 7 h$} & $3 / 2[7 / 2]$ & ${ }^{3} G$ & 3 & 366868.82 & 0.749 & 3.357 \\
\hline & $3 / 2[7 / 2]$ & ${ }^{3} G$ & 4 & 366871.21 & 1.028 & 3.373 \\
\hline & $3 / 2[13 / 2]$ & ${ }^{1} I$ & 6 & 366906.01 & 1.011 & 3.402 \\
\hline & $3 / 2[13 / 2]$ & ${ }^{3} I$ & 7 & 366906.17 & 1.143 & 3.395 \\
\hline & $3 / 2[9 / 2]$ & ${ }^{3} H$ & 4 & 367008.56 & 0.890 & 3.481 \\
\hline & $3 / 2[9 / 2]$ & ${ }^{3} H$ & 5 & 367008.82 & 1.092 & 3.474 \\
\hline & $3 / 2[11 / 2]$ & ${ }^{1} H$ & 5 & 367047.78 & 0.964 & 3.508 \\
\hline & $3 / 2[11 / 2]$ & ${ }^{3} H$ & 6 & 367049.05 & 1.124 & 3.510 \\
\hline & $1 / 2[11 / 2]$ & ${ }^{3} I$ & 5 & 388216.03 & 0.884 & 3.430 \\
\hline & $1 / 2[11 / 2]$ & ${ }^{3} I$ & 6 & 388217.67 & 1.056 & 3.434 \\
\hline & $1 / 2[9 / 2]$ & ${ }^{3} G$ & 5 & 388219.99 & 1.127 & 3.433 \\
\hline & $1 / 2[9 / 2]$ & ${ }^{1} G$ & 4 & 388220.01 & 0.932 & 3.447 \\
\hline \multirow[t]{4}{*}{$5 p^{5} 8 h$} & $3 / 2[7 / 2]$ & ${ }^{3} G$ & 3 & 375330.80 & 0.749 & 4.904 \\
\hline & $3 / 2[7 / 2]$ & ${ }^{3} G$ & 4 & 375332.41 & 1.028 & 4.921 \\
\hline & $3 / 2[13 / 2]$ & ${ }^{3} I$ & 7 & 375355.27 & 1.143 & 4.985 \\
\hline & $3 / 2[13 / 2]$ & ${ }^{1} I$ & 6 & 375355.59 & 1.011 & 4.999 \\
\hline
\end{tabular}

\begin{tabular}{|c|c|c|c|c|c|c|}
\hline \multirow{2}{*}{ Conf. } & \multicolumn{2}{|l|}{ Term } & \multirow{2}{*}{$\mathrm{J}$} & \multirow{2}{*}{$\frac{E\left[\mathrm{~cm}^{-1}\right]}{\mathrm{HFR}}$} & \multirow{2}{*}{$\frac{g \text {-factor }}{\text { HFR }}$} & \multirow{2}{*}{$\begin{array}{c}\tau[\mathrm{ns}] \\
\mathrm{HFR}\end{array}$} \\
\hline & $j K$ & $L S$ & & & & \\
\hline \multirow[t]{8}{*}{$5 p^{5} 8 h$} & $3 / 2[9 / 2]$ & ${ }^{3} H$ & 4 & 375452.37 & 0.894 & 4.455 \\
\hline & $3 / 2[9 / 2]$ & ${ }^{3} H$ & 5 & 375452.87 & 1.096 & 4.441 \\
\hline & $3 / 2[11 / 2]$ & ${ }^{1} H$ & 5 & 375466.37 & 0.959 & 4.749 \\
\hline & $3 / 2[11 / 2]$ & ${ }^{3} H$ & 6 & 375467.05 & 1.119 & 4.749 \\
\hline & $1 / 2[11 / 2]$ & ${ }^{3} I$ & 5 & 396646.34 & 0.884 & 5.023 \\
\hline & $1 / 2[11 / 2]$ & ${ }^{3} I$ & 6 & 396647.43 & 1.056 & 5.033 \\
\hline & $1 / 2[9 / 2]$ & ${ }^{3} G$ & 5 & 396648.59 & 1.127 & 5.021 \\
\hline & $1 / 2[9 / 2]$ & ${ }^{1} G$ & 4 & 396649.05 & 0.932 & 5.047 \\
\hline \multirow[t]{12}{*}{$5 p^{5} 9 h$} & $3 / 2[7 / 2]$ & ${ }^{3} G$ & 3 & 381136.62 & 0.749 & 6.929 \\
\hline & $3 / 2[7 / 2]$ & ${ }^{3} G$ & 4 & 381138.21 & 1.028 & 7.005 \\
\hline & $3 / 2[13 / 2]$ & ${ }^{3} I$ & 7 & 381153.66 & 1.143 & 7.047 \\
\hline & $3 / 2[13 / 2]$ & ${ }^{1} I$ & 6 & 381153.80 & 1.011 & 7.062 \\
\hline & $3 / 2[9 / 2]$ & ${ }^{3} H$ & 5 & 381202.78 & 1.092 & 7.045 \\
\hline & $3 / 2[9 / 2]$ & ${ }^{3} H$ & 4 & 381202.89 & 0.889 & 7.062 \\
\hline & $3 / 2[11 / 2]$ & ${ }^{1} H$ & 5 & 381221.07 & 0.964 & 7.092 \\
\hline & $3 / 2[11 / 2]$ & ${ }^{3} H$ & 6 & 381221.76 & 1.124 & 7.096 \\
\hline & $1 / 2[11 / 2]$ & ${ }^{3} I$ & 5 & 402433.32 & 0.884 & 7.048 \\
\hline & $1 / 2[11 / 2]$ & ${ }^{3} I$ & 6 & 402434.27 & 1.056 & 7.058 \\
\hline & $1 / 2[9 / 2]$ & ${ }^{3} G$ & 5 & 402435.02 & 1.127 & 7.002 \\
\hline & $1 / 2[9 / 2]$ & ${ }^{1} G$ & 4 & 402435.39 & 0.933 & 7.054 \\
\hline \multirow[t]{12}{*}{$5 p^{5} 10 h$} & $3 / 2[7 / 2]$ & ${ }^{3} G$ & 3 & 385288.24 & 0.749 & 9.034 \\
\hline & $3 / 2[7 / 2]$ & ${ }^{3} G$ & 4 & 385289.67 & 1.028 & 9.298 \\
\hline & $3 / 2[13 / 2]$ & ${ }^{3} I$ & 7 & 385300.56 & 1.143 & 9.822 \\
\hline & $3 / 2[13 / 2]$ & ${ }^{1} I$ & 6 & 385300.59 & 1.011 & 9.792 \\
\hline & $3 / 2[9 / 2]$ & ${ }^{3} H$ & 5 & 385335.56 & 1.092 & 9.062 \\
\hline & $3 / 2[9 / 2]$ & ${ }^{3} H$ & 4 & 385335.62 & 0.889 & 9.213 \\
\hline & $3 / 2[11 / 2]$ & ${ }^{1} H$ & 5 & 385349.23 & 0.964 & 9.252 \\
\hline & $3 / 2[11 / 2]$ & ${ }^{3} H$ & 6 & 385349.91 & 1.124 & 9.246 \\
\hline & $1 / 2[11 / 2]$ & ${ }^{3} I$ & 5 & 406573.01 & 0.884 & 9.576 \\
\hline & $1 / 2[11 / 2]$ & ${ }^{3} I$ & 6 & 406573.91 & 1.056 & 9.560 \\
\hline & $1 / 2[9 / 2]$ & ${ }^{3} G$ & 5 & 406574.39 & 1.127 & 8.986 \\
\hline & $1 / 2[9 / 2]$ & ${ }^{1} G$ & 4 & 406574.62 & 0.933 & 9.198 \\
\hline \multirow[t]{11}{*}{$5 p^{5} 7 d$} & $3 / 2[1 / 2]^{\circ}$ & ${ }^{3} P^{o}$ & 0 & 329885.49 & 0.000 & 1.385 \\
\hline & $3 / 2[1 / 2]^{o}$ & ${ }^{3} P^{o}$ & 1 & 330299.02 & 1.318 & 1.295 \\
\hline & $3 / 2[7 / 2]^{o}$ & ${ }^{3} F^{o}$ & 4 & 330731.78 & 1.249 & 1.525 \\
\hline & $3 / 2[7 / 2]^{\circ}$ & ${ }^{3} F^{o}$ & 3 & 330757.34 & 1.051 & 1.318 \\
\hline & $3 / 2[3 / 2]^{o}$ & ${ }^{3} D^{o}$ & 2 & 330916.55 & 1.049 & 1.001 \\
\hline & $3 / 2[5 / 2]^{\circ}$ & ${ }^{1} D^{o}$ & 2 & 331403.85 & 0.960 & 1.435 \\
\hline & $3 / 2[5 / 2]^{o}$ & ${ }^{3} D^{o}$ & 3 & 331659.55 & 1.108 & 1.075 \\
\hline & $3 / 2[3 / 2]^{o}$ & ${ }^{1} P^{o}$ & 1 & 333682.84 & 0.862 & 0.207 \\
\hline & $1 / 2[5 / 2]^{o}$ & ${ }^{3} F^{o}$ & 2 & 351755.14 & 0.772 & 1.375 \\
\hline & $1 / 2[3 / 2]^{o}$ & ${ }^{3} P^{o}$ & 2 & 351992.45 & 1.278 & 1.646 \\
\hline & $1 / 2[5 / 2]^{o}$ & ${ }^{1} F^{o}$ & 3 & 352167.66 & 1.109 & 1.499 \\
\hline \multirow[t]{9}{*}{$5 p^{5} 8 d$} & $1 / 2[3 / 2]^{o}$ & ${ }^{3} P^{o}$ & 1 & 352584.56 & 1.104 & 1.006 \\
\hline & $3 / 2[1 / 2]^{\circ}$ & ${ }^{3} P^{o}$ & 0 & 352865.79 & 0.000 & 2.641 \\
\hline & $3 / 2[7 / 2]^{\circ}$ & ${ }^{3} F^{o}$ & 4 & 353344.07 & 1.247 & 2.627 \\
\hline & $3 / 2[3 / 2]^{o}$ & ${ }^{3} D^{o}$ & 1 & 353423.58 & 1.005 & 1.424 \\
\hline & $3 / 2[7 / 2]^{\circ}$ & ${ }^{1} F^{o}$ & 3 & 353482.01 & 1.032 & 1.588 \\
\hline & $3 / 2[3 / 2]^{o}$ & ${ }^{3} D^{o}$ & 2 & 353621.28 & 1.292 & 2.483 \\
\hline & $3 / 2[5 / 2]^{\circ}$ & ${ }^{1} D^{o}$ & 2 & 353758.98 & 0.984 & 2.616 \\
\hline & $3 / 2[5 / 2]^{o}$ & ${ }^{3} D^{o}$ & 3 & 353901.52 & 1.235 & 2.717 \\
\hline & $3 / 2[1 / 2]^{o}$ & ${ }^{1} P^{o}$ & 1 & 355437.45 & 0.896 & 0.194 \\
\hline
\end{tabular}


TABLE I (cont.)

TABLE I (cont.)

\begin{tabular}{|c|c|c|c|c|c|c|}
\hline \multirow{2}{*}{ Conf. } & \multicolumn{2}{|l|}{ Term } & \multirow[b]{2}{*}{$J$} & \multirow{2}{*}{\begin{tabular}{|c|}
$E\left[\mathrm{~cm}^{-1}\right]$ \\
$\mathrm{HFR}$
\end{tabular}} & \multirow{2}{*}{$\frac{g \text {-factor }}{\text { HFR }}$} & \multirow{2}{*}{$\begin{array}{c}\tau[\mathrm{ns}] \\
\mathrm{HFR}\end{array}$} \\
\hline & $j K$ & $L S$ & & & & \\
\hline \multirow[t]{3}{*}{$5 p^{5} 8 d$} & $1 / 2[5 / 2]^{o}$ & ${ }^{3} F^{o}$ & 2 & 374487.53 & 0.767 & 2.560 \\
\hline & $1 / 2[3 / 2]^{o}$ & ${ }^{3} P^{o}$ & 2 & 374627.64 & 1.285 & 2.852 \\
\hline & $1 / 2[5 / 2]^{o}$ & ${ }^{3} F^{o}$ & 3 & 374706.65 & 1.101 & 2.661 \\
\hline \multirow[t]{12}{*}{$5 p^{5} 9 d$} & $3 / 2[1 / 2]^{\circ}$ & ${ }^{3} P^{o}$ & 0 & 366421.43 & 0.000 & 4.209 \\
\hline & $3 / 2[1 / 2]^{o}$ & ${ }^{3} P^{o}$ & 1 & 366546.40 & 1.309 & 4.000 \\
\hline & $3 / 2[7 / 2]^{o}$ & ${ }^{3} F^{o}$ & 4 & 366721.82 & 1.217 & 3.234 \\
\hline & $3 / 2[3 / 2]^{o}$ & ${ }^{3} D^{o}$ & 2 & 366748.82 & 1.311 & 4.338 \\
\hline & $3 / 2[7 / 2]^{o}$ & ${ }^{1} F^{o}$ & 3 & 366767.02 & 1.000 & 2.762 \\
\hline & $3 / 2[5 / 2]^{o}$ & ${ }^{1} D^{o}$ & 2 & 366904.38 & 0.968 & 4.280 \\
\hline & $3 / 2[5 / 2]^{o}$ & ${ }^{3} D^{o}$ & 3 & 367016.42 & 1.230 & 4.361 \\
\hline & $3 / 2[3 / 2]^{o}$ & ${ }^{1} P^{o}$ & 1 & 367614.60 & 0.865 & 6.415 \\
\hline & $1 / 2[5 / 2]^{o}$ & ${ }^{3} F^{o}$ & 2 & 387917.56 & 0.765 & 4.279 \\
\hline & $1 / 2[3 / 2]^{o}$ & ${ }^{3} P^{o}$ & 2 & 388019.37 & 1.288 & 4.540 \\
\hline & $1 / 2[5 / 2]^{o}$ & ${ }^{3} F^{o}$ & 3 & 388084.69 & 1.109 & 4.480 \\
\hline & $1 / 2[3 / 2]^{o}$ & ${ }^{3} D^{o}$ & 1 & 388419.56 & 0.834 & 5.182 \\
\hline \multirow[t]{13}{*}{$5 p^{5} 10 d$} & $3 / 2[1 / 2]^{\circ}$ & ${ }^{3} P^{o}$ & 1 & 374974.88 & 1.150 & 1.452 \\
\hline & $3 / 2[1 / 2]^{o}$ & ${ }^{3} P^{o}$ & 0 & 375083.43 & 0.000 & 4.313 \\
\hline & $3 / 2[7 / 2]^{o}$ & ${ }^{3} F^{o}$ & 4 & 375283.35 & 1.226 & 4.203 \\
\hline & $3 / 2[3 / 2]^{o}$ & ${ }^{3} D^{o}$ & 1 & 375326.34 & 0.955 & 2.290 \\
\hline & $3 / 2[7 / 2]^{o}$ & ${ }^{1} F^{o}$ & 3 & 375344.52 & 1.026 & 4.387 \\
\hline & $3 / 2[3 / 2]^{o}$ & ${ }^{3} D^{o}$ & 2 & 375389.75 & 1.269 & 5.240 \\
\hline & $3 / 2[5 / 2]^{\circ}$ & ${ }^{1} D^{o}$ & 2 & 375440.65 & 1.005 & 6.309 \\
\hline & $3 / 2[5 / 2]^{\circ}$ & ${ }^{3} D^{o}$ & 3 & 375498.65 & 1.242 & 6.947 \\
\hline & $3 / 2[1 / 2]^{o}$ & ${ }^{1} P^{o}$ & 1 & 376262.11 & 0.897 & 0.453 \\
\hline & $1 / 2[5 / 2]^{o}$ & ${ }^{3} F^{o}$ & 2 & 396495.10 & 0.765 & 5.182 \\
\hline & $1 / 2[3 / 2]^{o}$ & ${ }^{3} P^{o}$ & 2 & 396567.08 & 1.289 & 5.102 \\
\hline & $1 / 2[5 / 2]^{o}$ & ${ }^{3} F^{o}$ & 3 & 396611.22 & 1.110 & 6.074 \\
\hline & $1 / 2[3 / 2]^{o}$ & ${ }^{3} D^{o}$ & 1 & 396839.43 & 0.832 & 1.982 \\
\hline \multirow[t]{4}{*}{$5 p^{5} 9 s$} & $3 / 2[3 / 2]^{\circ}$ & ${ }^{3} P^{o}$ & 2 & 354488.66 & 1.501 & 1.450 \\
\hline & $3 / 2[3 / 2]^{\circ}$ & ${ }^{1} P^{o}$ & 1 & 354725.83 & 1.193 & 0.404 \\
\hline & $1 / 2[1 / 2]^{o}$ & ${ }^{3} P^{o}$ & 0 & 375708.00 & 0.000 & 1.420 \\
\hline & $1 / 2[1 / 2]^{o}$ & ${ }^{3} P^{o}$ & 1 & 375798.39 & 1.331 & 0.895 \\
\hline \multirow[t]{4}{*}{$5 p^{5} 10 s$} & $3 / 2[3 / 2]^{\circ}$ & ${ }^{3} P^{o}$ & 2 & 367357.50 & 1.501 & 2.129 \\
\hline & $3 / 2[3 / 2]^{o}$ & ${ }^{1} P^{o}$ & 1 & 367457.19 & 1.159 & 0.763 \\
\hline & $1 / 2[1 / 2]^{o}$ & ${ }^{3} P^{o}$ & 0 & 388591.19 & 0.000 & 2.121 \\
\hline & $1 / 2[1 / 2]^{o}$ & ${ }^{3} P^{o}$ & 1 & 388644.81 & 1.332 & 1.397 \\
\hline \multirow[t]{12}{*}{$5 p^{5} 5 g$} & $3 / 2[5 / 2]^{\circ}$ & ${ }^{3} F^{o}$ & 2 & 330948.67 & 0.944 & 0.895 \\
\hline & $3 / 2[5 / 2]^{o}$ & ${ }^{3} F^{o}$ & 3 & 331020.88 & 1.062 & 0.744 \\
\hline & $3 / 2[11 / 2]^{o}$ & ${ }^{3} H^{o}$ & 6 & 331185.08 & 1.167 & 0.714 \\
\hline & $3 / 2[11 / 2]^{o}$ & ${ }^{1} H^{o}$ & 5 & 331220.66 & 1.016 & 0.711 \\
\hline & $3 / 2[7 / 2]^{o}$ & ${ }^{3} F^{o}$ & 4 & 331691.33 & 1.129 & 0.745 \\
\hline & $3 / 2[7 / 2]^{o}$ & ${ }^{1} F^{o}$ & 3 & 331829.17 & 1.007 & 0.967 \\
\hline & $3 / 2[9 / 2]^{o}$ & ${ }^{1} G^{o}$ & 4 & 331942.96 & 0.958 & 0.740 \\
\hline & $3 / 2[9 / 2]^{o}$ & ${ }^{3} G^{o}$ & 5 & 331957.18 & 1.148 & 0.755 \\
\hline & $1 / 2[7 / 2]^{o}$ & ${ }^{3} G^{o}$ & 4 & 352578.46 & 1.149 & 0.741 \\
\hline & $1 / 2[9 / 2]^{o}$ & ${ }^{3} H^{o}$ & 4 & 352635.86 & 0.869 & 0.706 \\
\hline & $1 / 2[7 / 2]^{o}$ & ${ }^{3} G^{o}$ & 3 & 352639.22 & 0.913 & 0.769 \\
\hline & $1 / 2[9 / 2]^{\circ}$ & ${ }^{3} H^{o}$ & 5 & 352667.91 & 1.075 & 0.726 \\
\hline \multirow[t]{4}{*}{$5 p^{5} 6 g$} & $3 / 2[5 / 2]^{\circ}$ & ${ }^{3} F^{o}$ & 2 & 353124.43 & 0.670 & 1.120 \\
\hline & $3 / 2[5 / 2]^{o}$ & ${ }^{3} F^{o}$ & 3 & 353198.33 & 1.050 & 1.423 \\
\hline & $3 / 2[11 / 2]^{o}$ & ${ }^{3} H^{o}$ & 6 & 353200.57 & 1.167 & 1.126 \\
\hline & $3 / 2[11 / 2]^{o}$ & ${ }^{1} H^{o}$ & 5 & 353230.71 & 1.015 & 1.131 \\
\hline
\end{tabular}

\begin{tabular}{|c|c|c|c|c|c|c|}
\hline \multirow{2}{*}{ Conf. } & \multicolumn{2}{|l|}{ Term } & \multirow{2}{*}{$J$} & \multirow{2}{*}{$\begin{array}{c}E\left[\mathrm{~cm}^{-1}\right] \\
\mathrm{HFR}\end{array}$} & \multirow{2}{*}{\begin{tabular}{|c|}
$g$-factor \\
HFR
\end{tabular}} & \multirow{2}{*}{\begin{tabular}{|l}
$\tau[\mathrm{ns}$ \\
$\mathrm{HFR}$
\end{tabular}} \\
\hline & $j K$ & $L S$ & & & & \\
\hline \multirow[t]{8}{*}{$5 p^{5} 6 g$} & $3 / 2[7 / 2]^{\circ}$ & ${ }^{3} F^{o}$ & 4 & 353634.98 & 1.130 & 1.111 \\
\hline & $3 / 2[7 / 2]^{\circ}$ & ${ }^{3} G^{o}$ & 3 & 353674.25 & 0.910 & 1.250 \\
\hline & $3 / 2[9 / 2]^{\circ}$ & ${ }^{1} G^{o}$ & 4 & 353710.38 & 0.955 & 1.123 \\
\hline & $3 / 2[9 / 2]^{\circ}$ & ${ }^{3} G^{o}$ & 5 & 353712.19 & 1.145 & 1.145 \\
\hline & $1 / 2[7 / 2]^{o}$ & ${ }^{3} G^{o}$ & 4 & 374557.82 & 1.152 & 1.164 \\
\hline & $1 / 2[9 / 2]^{o}$ & ${ }^{3} H^{o}$ & 4 & 374574.79 & 0.866 & 1.112 \\
\hline & $1 / 2[9 / 2]^{o}$ & ${ }^{3} H^{o}$ & 5 & 374594.06 & 1.072 & 1.147 \\
\hline & $1 / 2[7 / 2]^{\circ}$ & ${ }^{1} F^{o}$ & 3 & 374614.19 & 0.919 & 1.236 \\
\hline \multirow[t]{12}{*}{$5 p^{5} 7 g$} & $3 / 2[5 / 2]^{\circ}$ & ${ }^{3} F^{o}$ & 2 & 366371.44 & 0.668 & 1.638 \\
\hline & $3 / 2[5 / 2]^{\circ}$ & ${ }^{3} F^{o}$ & 3 & 366417.01 & 1.053 & 1.760 \\
\hline & $3 / 2[11 / 2]^{o}$ & ${ }^{3} H^{o}$ & 6 & 366461.35 & 1.167 & 1.682 \\
\hline & $3 / 2[11 / 2]^{o}$ & ${ }^{1} H^{o}$ & 5 & 366486.19 & 1.016 & 1.690 \\
\hline & $3 / 2[7 / 2]^{o}$ & ${ }^{3} F^{o}$ & 4 & 366609.87 & 1.157 & 2.010 \\
\hline & $3 / 2[7 / 2]^{o}$ & ${ }^{3} G^{o}$ & 3 & 366610.83 & 0.942 & 2.300 \\
\hline & $3 / 2[9 / 2]^{o}$ & ${ }^{1} G^{o}$ & 4 & 366734.73 & 0.961 & 1.691 \\
\hline & $3 / 2[9 / 2]^{\circ}$ & ${ }^{3} G^{o}$ & 5 & 366742.58 & 1.149 & 1.729 \\
\hline & $1 / 2[9 / 2]^{o}$ & ${ }^{3} H^{o}$ & 4 & 387819.24 & 0.861 & 1.615 \\
\hline & $1 / 2[7 / 2]^{o}$ & ${ }^{3} G^{o}$ & 4 & 387832.86 & 1.155 & 1.644 \\
\hline & $1 / 2[9 / 2]^{o}$ & ${ }^{3} H^{o}$ & 5 & 387835.34 & 1.068 & 1.664 \\
\hline & $1 / 2[7 / 2]^{o}$ & ${ }^{1} F^{o}$ & 3 & 387868.47 & 0.917 & 1.705 \\
\hline \multirow[t]{12}{*}{$5 p^{5} 8 g$} & $3 / 2[5 / 2]^{\circ}$ & ${ }^{3} F^{o}$ & 2 & 375033.15 & 0.671 & 2.399 \\
\hline & $3 / 2[11 / 2]^{o}$ & ${ }^{3} H^{o}$ & 6 & 375062.57 & 1.167 & 2.419 \\
\hline & $3 / 2[11 / 2]^{o}$ & ${ }^{1} H^{o}$ & 5 & 375079.58 & 1.016 & 2.427 \\
\hline & $3 / 2[5 / 2]^{o}$ & ${ }^{3} F^{o}$ & 3 & 375106.50 & 1.056 & 2.707 \\
\hline & $3 / 2[7 / 2]^{o}$ & ${ }^{3} G^{o}$ & 4 & 375233.30 & 1.148 & 2.453 \\
\hline & $3 / 2[7 / 2]^{\circ}$ & ${ }^{3} G^{o}$ & 3 & 375235.36 & 0.905 & 2.409 \\
\hline & $3 / 2[9 / 2]^{\circ}$ & ${ }^{1} G^{o}$ & 4 & 375277.09 & 0.958 & 2.277 \\
\hline & $3 / 2[9 / 2]^{\circ}$ & ${ }^{3} G^{o}$ & 5 & 375279.11 & 1.148 & 2.323 \\
\hline & $1 / 2[9 / 2]^{\circ}$ & ${ }^{3} H^{o}$ & 4 & 396377.58 & 0.860 & 2.298 \\
\hline & $1 / 2[7 / 2]^{o}$ & ${ }^{3} F^{o}$ & 4 & 396384.94 & 1.156 & 2.329 \\
\hline & $1 / 2[9 / 2]^{\circ}$ & ${ }^{3} H^{o}$ & 5 & 396389.02 & 1.068 & 2.366 \\
\hline & $1 / 2[7 / 2]^{o}$ & ${ }^{1} F^{o}$ & 3 & 396417.10 & 0.917 & 2.398 \\
\hline \multirow[t]{12}{*}{$5 p^{5} 9 g$} & $3 / 2[5 / 2]^{\circ}$ & ${ }^{3} F^{o}$ & 2 & 380905.30 & 0.666 & 3.337 \\
\hline & $3 / 2[5 / 2]^{\circ}$ & ${ }^{3} F^{o}$ & 3 & 380932.24 & 1.051 & 3.468 \\
\hline & $3 / 2[11 / 2]^{o}$ & ${ }^{3} H^{o}$ & 6 & 380943.72 & 1.167 & 3.398 \\
\hline & $3 / 2[11 / 2]^{o}$ & ${ }^{1} H^{o}$ & 5 & 380957.06 & 1.017 & 3.374 \\
\hline & $3 / 2[7 / 2]^{o}$ & ${ }^{3} G^{o}$ & 4 & 381030.27 & 1.122 & 3.284 \\
\hline & $3 / 2[7 / 2]^{\circ}$ & ${ }^{3} G^{o}$ & 3 & 381054.13 & 0.867 & 3.331 \\
\hline & $3 / 2[9 / 2]^{\circ}$ & ${ }^{1} G^{o}$ & 4 & 381074.20 & 0.962 & 3.212 \\
\hline & $3 / 2[9 / 2]^{o}$ & ${ }^{3} G^{o}$ & 5 & 381078.38 & 1.150 & 3.267 \\
\hline & $1 / 2[9 / 2]^{o}$ & ${ }^{3} H^{o}$ & 4 & 402241.93 & 0.860 & 3.185 \\
\hline & $1 / 2[7 / 2]^{o}$ & ${ }^{3} F^{o}$ & 4 & 402246.40 & 1.157 & 3.204 \\
\hline & $1 / 2[9 / 2]^{\circ}$ & ${ }^{3} H^{o}$ & 5 & 402251.07 & 1.067 & 3.255 \\
\hline & $1 / 2[7 / 2]^{\circ}$ & ${ }^{1} F^{o}$ & 3 & 402270.51 & 0.915 & 3.222 \\
\hline \multirow[t]{8}{*}{$5 p^{5} 10 g$} & $3 / 2[5 / 2]^{\circ}$ & ${ }^{3} F^{o}$ & 2 & 385119.72 & 0.666 & 3.206 \\
\hline & $3 / 2[5 / 2]^{\circ}$ & ${ }^{3} F^{o}$ & 3 & 385137.74 & 1.051 & 4.004 \\
\hline & $3 / 2[11 / 2]^{o}$ & ${ }^{3} H^{o}$ & 6 & 385148.28 & 1.167 & 3.847 \\
\hline & $3 / 2[11 / 2]^{o}$ & ${ }^{1} H^{o}$ & 5 & 385158.15 & 1.017 & 4.499 \\
\hline & $3 / 2[7 / 2]^{\circ}$ & ${ }^{3} G^{o}$ & 4 & 385206.11 & 1.122 & 3.655 \\
\hline & $3 / 2[7 / 2]^{o}$ & ${ }^{3} G^{o}$ & 3 & 385226.20 & 0.867 & 5.373 \\
\hline & $3 / 2[9 / 2]^{\circ}$ & ${ }^{1} G^{o}$ & 4 & 385240.78 & 0.962 & 3.944 \\
\hline & $3 / 2[9 / 2]^{\circ}$ & ${ }^{3} G^{o}$ & 5 & 385244.63 & 1.150 & 4.197 \\
\hline
\end{tabular}


TABLE I (cont.)

\begin{tabular}{|c|c|c|c|c|c|c|}
\hline \multirow{2}{*}{ Conf. } & \multicolumn{2}{|l|}{ Term } & \multirow[b]{2}{*}{$J$} & \multirow{2}{*}{$\begin{array}{c}E\left[\mathrm{~cm}^{-1}\right] \\
\mathrm{HFR}\end{array}$} & \multirow{2}{*}{$\begin{array}{c}g \text {-factor } \\
\text { HFR }\end{array}$} & \multirow{2}{*}{$\begin{array}{c}\tau[\mathrm{ns}] \\
\mathrm{HFR}\end{array}$} \\
\hline & $j K$ & $L S$ & & & & \\
\hline \multirow[t]{4}{*}{$5 p^{5} 10 g$} & $1 / 2[9 / 2]^{o}$ & ${ }^{3} H^{o}$ & 4 & 406433.78 & 0.859 & 3.697 \\
\hline & $1 / 2[7 / 2]^{o}$ & ${ }^{3} F^{o}$ & 4 & 406436.94 & 1.157 & 3.310 \\
\hline & $1 / 2[9 / 2]^{o}$ & ${ }^{3} H^{o}$ & 5 & 406440.91 & 1.067 & 4.182 \\
\hline & $1 / 2[7 / 2]^{o}$ & ${ }^{1} F^{o}$ & 3 & 406455.27 & 0.916 & 4.336 \\
\hline \multirow[t]{12}{*}{$5 p^{5} 7 i$} & $3 / 2[9 / 2]^{o}$ & ${ }^{3} H^{O}$ & 4 & 367024.64 & 0.800 & 5.142 \\
\hline & $3 / 2[9 / 2]^{\circ}$ & ${ }^{3} H^{o}$ & 5 & 367025.37 & 1.018 & 5.143 \\
\hline & $3 / 2[15 / 2]^{o}$ & ${ }^{1} K^{o}$ & 7 & 367043.04 & 1.008 & 5.164 \\
\hline & $3 / 2[15 / 2]^{o}$ & ${ }^{3} K^{o}$ & 8 & 367043.68 & 1.125 & 5.165 \\
\hline & $3 / 2[11 / 2]^{o}$ & ${ }^{3} I^{o}$ & 5 & 367107.63 & 0.904 & 5.226 \\
\hline & $3 / 2[11 / 2]^{o}$ & ${ }^{3} I^{o}$ & 6 & 367108.20 & 1.073 & 5.227 \\
\hline & $3 / 2[13 / 2]^{o}$ & ${ }^{1} I^{o}$ & 6 & 367127.16 & 0.967 & 5.245 \\
\hline & $3 / 2[13 / 2]^{o}$ & ${ }^{3} I^{o}$ & 7 & 367127.79 & 1.105 & 5.246 \\
\hline & $1 / 2[13 / 2]^{o}$ & ${ }^{3} K^{o}$ & 6 & 388331.41 & 0.901 & 5.197 \\
\hline & $1 / 2[11 / 2]^{o}$ & ${ }^{1} H^{o}$ & 5 & 388332.02 & 0.944 & 5.197 \\
\hline & $1 / 2[13 / 2]^{o}$ & ${ }^{3} K^{o}$ & 7 & 388332.06 & 1.048 & 5.198 \\
\hline & $1 / 2[11 / 2]^{o}$ & ${ }^{3} H^{o}$ & 6 & 388332.60 & 1.107 & 5.198 \\
\hline \multirow[t]{12}{*}{$5 p^{5} 8 i$} & $3 / 2[9 / 2]^{\circ}$ & ${ }^{3} H^{o}$ & 4 & 375441.55 & 0.800 & 7.566 \\
\hline & $3 / 2[9 / 2]^{o}$ & ${ }^{3} H^{o}$ & 5 & 375442.27 & 1.018 & 7.569 \\
\hline & $3 / 2[15 / 2]^{o}$ & ${ }^{1} K^{o}$ & 7 & 375453.73 & 1.008 & 7.612 \\
\hline & $3 / 2[15 / 2]^{o}$ & ${ }^{3} K^{o}$ & 8 & 375454.37 & 1.125 & 7.612 \\
\hline & $3 / 2[11 / 2]^{o}$ & ${ }^{3} I^{o}$ & 5 & 375497.11 & 0.904 & 7.706 \\
\hline & $3 / 2[11 / 2]^{o}$ & ${ }^{3} I^{o}$ & 6 & 375497.71 & 1.073 & 7.706 \\
\hline & $3 / 2[13 / 2]^{o}$ & ${ }^{1} I^{o}$ & 6 & 375510.26 & 0.967 & 7.734 \\
\hline & $3 / 2[13 / 2]^{o}$ & ${ }^{3} I^{o}$ & 7 & 375510.91 & 1.105 & 7.735 \\
\hline & $1 / 2[13 / 2]^{o}$ & ${ }^{3} K^{o}$ & 6 & 396730.81 & 0.901 & 7.659 \\
\hline & $1 / 2[11 / 2]^{o}$ & ${ }^{1} H^{o}$ & 5 & 396731.24 & 0.944 & 7.661 \\
\hline & $1 / 2[13 / 2]^{o}$ & ${ }^{3} K^{o}$ & 7 & 396731.49 & 1.048 & 7.661 \\
\hline & $1 / 2[11 / 2]^{o}$ & ${ }^{3} H^{o}$ & 6 & 396731.86 & 1.107 & 7.661 \\
\hline \multirow[t]{12}{*}{$5 p^{5} 9 i$} & $3 / 2[9 / 2]^{\circ}$ & ${ }^{3} H^{o}$ & 4 & 381216.19 & 0.800 & 10.70 \\
\hline & $3 / 2[9 / 2]^{o}$ & ${ }^{3} H^{o}$ & 5 & 381216.85 & 1.018 & 10.70 \\
\hline & $3 / 2[15 / 2]^{o}$ & ${ }^{1} K^{o}$ & 7 & 381224.75 & 1.008 & 10.74 \\
\hline & $3 / 2[15 / 2]^{o}$ & ${ }^{3} K^{o}$ & 8 & 381225.37 & 1.125 & 10.74 \\
\hline & $3 / 2[11 / 2]^{o}$ & ${ }^{3} I^{o}$ & 5 & 381255.28 & 0.904 & 10.84 \\
\hline & $3 / 2[11 / 2]^{o}$ & ${ }^{3} I^{o}$ & 6 & 381255.89 & 1.073 & 10.84 \\
\hline & $3 / 2[13 / 2]^{o}$ & ${ }^{1} I^{o}$ & 6 & 381264.60 & 0.967 & 10.87 \\
\hline & $3 / 2[13 / 2]^{o}$ & ${ }^{3} I^{o}$ & 7 & 381265.23 & 1.105 & 10.87 \\
\hline & $1 / 2\left[13 / 2^{\circ}\right]$ & ${ }^{3} K^{o}$ & 6 & 402495.03 & 0.901 & 10.79 \\
\hline & $1 / 2[11 / 2]^{o}$ & ${ }^{1} H^{o}$ & 5 & 402495.32 & 0.944 & 10.79 \\
\hline & $1 / 2[13 / 2]^{o}$ & ${ }^{3} K^{o}$ & 7 & 402495.69 & 1.048 & 10.79 \\
\hline & $1 / 2[11 / 2]^{o}$ & ${ }^{3} H^{o}$ & 6 & 402495.95 & 1.107 & 10.79 \\
\hline \multirow[t]{12}{*}{$5 p^{5} 10 i$} & $3 / 2[9 / 2]^{\circ}$ & ${ }^{3} H^{o}$ & 4 & 385345.16 & 0.800 & 14.70 \\
\hline & $3 / 2[9 / 2]^{\circ}$ & ${ }^{3} H^{o}$ & 5 & 385345.18 & 1.018 & 14.71 \\
\hline & $3 / 2[15 / 2]^{o}$ & ${ }^{3} K^{o}$ & 8 & 385351.32 & 1.125 & 14.73 \\
\hline & $3 / 2[15 / 2]^{o}$ & ${ }^{1} K^{o}$ & 7 & 385351.34 & 1.008 & 14.73 \\
\hline & $3 / 2[11 / 2]^{o}$ & ${ }^{3} I^{o}$ & 6 & 385373.55 & 1.073 & 14.64 \\
\hline & $3 / 2[11 / 2]^{o}$ & ${ }^{3} I^{o}$ & 5 & 385373.56 & 0.904 & 14.64 \\
\hline & $3 / 2[13 / 2]^{o}$ & ${ }^{1} I^{o}$ & 6 & 385380.42 & 0.967 & 14.63 \\
\hline & $3 / 2[13 / 2]^{o}$ & ${ }^{3} I^{o}$ & 7 & 385380.43 & 1.105 & 14.63 \\
\hline & $1 / 2[13 / 2]^{o}$ & ${ }^{3} K^{o}$ & 6 & 406617.24 & 0.901 & 14.69 \\
\hline & $1 / 2[13 / 2]^{o}$ & ${ }^{3} K^{o}$ & 7 & 406617.25 & 1.048 & 14.69 \\
\hline & $1 / 2[11 / 2]^{o}$ & ${ }^{3} H^{o}$ & 6 & 406617.45 & 1.107 & 14.65 \\
\hline & $1 / 2[11 / 2]^{o}$ & ${ }^{1} H^{o}$ & 5 & 406617.47 & 0.944 & 14.65 \\
\hline
\end{tabular}


Energies $E$, Landé $g$-factors, and lifetimes $\tau$ for valence excitation states in La IV.

TABLE II

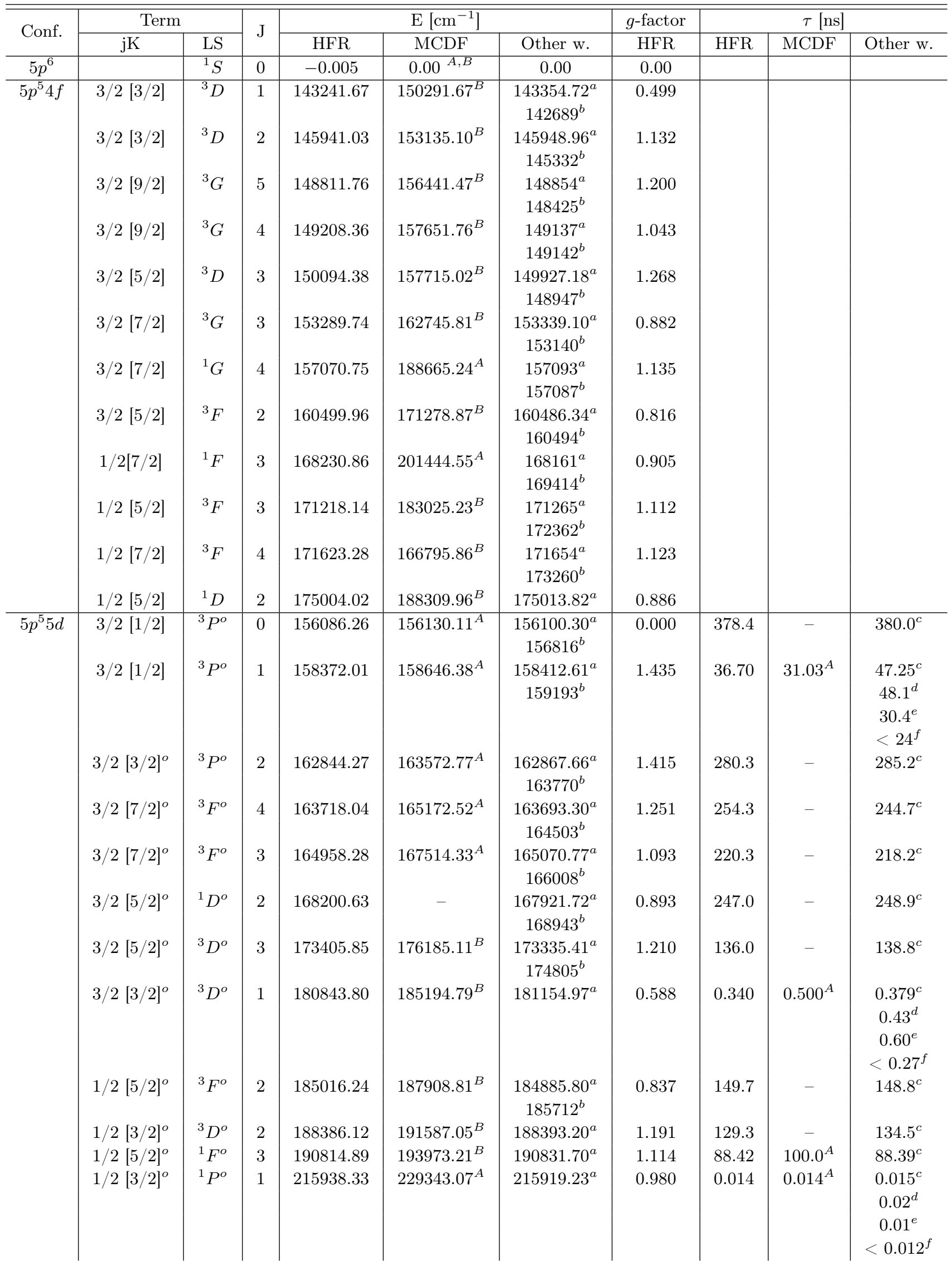


TABLE II (cont.)

\begin{tabular}{|c|c|c|c|c|c|c|c|c|c|c|}
\hline \multirow{2}{*}{ Conf. } & \multicolumn{2}{|c|}{ Term } & \multirow{2}{*}{$\mathrm{J}$} & \multicolumn{3}{|c|}{$\mathrm{E}\left[\mathrm{cm}^{-1}\right]$} & \multirow{2}{*}{$\frac{g \text {-factor }}{\text { HFR }}$} & \multicolumn{3}{|c|}{$\tau[\mathrm{ns}]$} \\
\hline & $\mathrm{jK}$ & $\mathrm{LS}$ & & HFR & MCDF & Other w. & & HFR & $\mathrm{MCDF}$ & Other w. \\
\hline \multirow[t]{10}{*}{$5 p^{5} 6 s$} & $3 / 2[3 / 2]^{\circ}$ & ${ }^{3} P^{o}$ & 2 & 198319.69 & $201077.89^{B}$ & $198303.15^{a}$ & 1.499 & 312.0 & - & $244.2^{c}$ \\
\hline & $3 / 2[3 / 2]^{o}$ & ${ }^{1} P^{o}$ & 1 & 200167.35 & $203665.23^{B}$ & $200183.26^{a}$ & 1.203 & 0.209 & $0.244^{B}$ & $0.218^{c}$ \\
\hline & & & & & & & & & & $0.27^{d}$ \\
\hline & & & & & & & & & & $0.21^{e}$ \\
\hline & & & & & & & & & & $0.26^{f}$ \\
\hline & $1 / 2[1 / 2]^{o}$ & ${ }^{3} P^{o}$ & 0 & 219313.38 & $218117.22^{A}$ & $219336.85^{a}$ & 0.000 & 194.3 & - & $185.2^{c}$ \\
\hline & $1 / 2[1 / 2]^{o}$ & ${ }^{3} P^{o}$ & 1 & 220530.57 & $219371.55^{A}$ & $220506.85^{a}$ & 1.296 & 0.130 & $0.156^{A}$ & $0.124^{c}$ \\
\hline & & & & & & & & & & $0.10^{d}$ \\
\hline & & & & & & & & & & $0.18^{e}$ \\
\hline & & & & & & & & & & $0.29^{f}$ \\
\hline \multirow[t]{20}{*}{$5 p^{5} 6 p$} & $3 / 2[1 / 2]$ & ${ }^{3} S$ & 1 & 231051.11 & $226405.88^{A}$ & $231008.71^{a}$ & 1.821 & 0.580 & $0.622^{A}$ & $0.572^{c}$ \\
\hline & & & & & & & & & & $0.626^{f}$ \\
\hline & $3 / 2[5 / 2]$ & ${ }^{3} D$ & 2 & 233281.75 & $229779.54^{A}$ & $233401.52^{a}$ & 1.148 & 0.659 & $0.742^{A}$ & $0.657^{c}$ \\
\hline & & & & & & & & & & $0.692^{f}$ \\
\hline & $3 / 2[5 / 2]$ & ${ }^{3} D$ & 3 & 236716.86 & $232553.75^{A}$ & $236790.42^{a}$ & 1.334 & 0.517 & $0.591^{A}$ & $0.524^{c}$ \\
\hline & & & & & & & & & & $0.539^{f}$ \\
\hline & $3 / 2[3 / 2]$ & ${ }^{1} P$ & 1 & 237750.24 & $234071.24^{A}$ & $237727.30^{a}$ & 1.071 & 0.616 & $0.743^{A}$ & $0.613^{c}$ \\
\hline & & & & & & & & & & $0.649^{f}$ \\
\hline & $3 / 2[3 / 2]$ & ${ }^{3} P$ & 2 & 239722.15 & $235848.52^{A}$ & $239654.29^{a}$ & 1.345 & 0.564 & $0.663^{A}$ & $0.575^{c}$ \\
\hline & & & & & & & & & & $0.552^{f}$ \\
\hline & $3 / 2[1 / 2]$ & ${ }^{3} P$ & 0 & 244361.33 & $241648.16^{A}$ & $244301.79^{a}$ & 0.000 & 0.636 & $0.779^{A}$ & $0.613^{c}$ \\
\hline & & & & & & & & & & $0.633^{f}$ \\
\hline & $1 / 2[3 / 2]$ & ${ }^{3} D$ & 1 & 254370.50 & $251333.06^{A}$ & $254251.34^{a}$ & 0.662 & 0.620 & $0.725^{A}$ & $0.633^{c}$ \\
\hline & & & & & & & & & & $0.672^{f}$ \\
\hline & $1 / 2[1 / 2]$ & ${ }^{3} P$ & 1 & 259461.42 & $255643.04^{A}$ & $259085.89^{a}$ & 1.448 & 0.525 & $0.338^{A}$ & $0.544^{c}$ \\
\hline & & & & & & & & & & $0.534^{f}$ \\
\hline & $1 / 2[3 / 2]$ & ${ }^{1} D$ & 2 & 259610.76 & - & $259224.33^{a}$ & 1.175 & 0.529 & - & $0.547^{c}$ \\
\hline & & & & & & & & & & $0.560^{f}$ \\
\hline & $1 / 2[1 / 2]$ & ${ }^{1} S$ & 0 & 260402.30 & $258703.90^{A}$ & $260449.83^{a}$ & 0.000 & 0.763 & $0.901^{A}$ & $0.724^{c}$ \\
\hline & & & & & & & & & & $0.789^{f}$ \\
\hline \multirow[t]{12}{*}{$5 p^{5} 6 d$} & $3 / 2[1 / 2]^{\circ}$ & ${ }^{3} P^{o}$ & 0 & 284212.11 & $278354.92^{A}$ & $284206.15^{a}$ & 0.000 & 0.603 & $0.643^{A}$ & $0.600^{c}$ \\
\hline & $3 / 2[1 / 2]^{o}$ & ${ }^{3} P^{o}$ & 1 & 284947.04 & $279556.04^{A}$ & $284981.97^{a}$ & 1.362 & 0.563 & $0.581^{A}$ & $0.507^{c}$ \\
\hline & $3 / 2[7 / 2]^{o}$ & ${ }^{3} F^{o}$ & 4 & 285815.05 & $281028.27^{A}$ & $285856.18^{a}$ & 1.251 & 0.756 & $0.693^{A}$ & $0.753^{c}$ \\
\hline & $3 / 2[7 / 2]^{o}$ & ${ }^{3} F^{o}$ & 3 & 286125.23 & $281741.70^{A}$ & $285951.00^{a}$ & 1.059 & 0.627 & $0.616^{A}$ & $0.629^{c}$ \\
\hline & $3 / 2[3 / 2]^{o}$ & ${ }^{3} P^{o}$ & 2 & 286142.87 & $281465.27^{A}$ & $286298.26^{a}$ & 1.341 & 0.715 & $0.665^{A}$ & $0.712^{c}$ \\
\hline & $3 / 2[5 / 2]^{\circ}$ & ${ }^{1} D^{o}$ & 2 & 287355.95 & $283523.87^{A}$ & $287402.66^{a}$ & 0.955 & 0.663 & $0.619^{A}$ & $0.661^{c}$ \\
\hline & $3 / 2[5 / 2]^{\circ}$ & ${ }^{3} D^{o}$ & 3 & 287833.87 & $284639.21^{A}$ & $287874.01^{a}$ & 1.239 & 0.786 & $0.650^{A}$ & $0.781^{c}$ \\
\hline & $3 / 2[3 / 2]^{o}$ & ${ }^{1} P^{o}$ & 1 & 290739.57 & $315638.54^{A}$ & $290596.17^{a}$ & 0.810 & 0.070 & $0.186^{A}$ & $0.035^{c}$ \\
\hline & $1 / 2[5 / 2]^{o}$ & ${ }^{3} F^{o}$ & 2 & 307238.64 & $303282.36^{A}$ & $307182.98^{a}$ & 0.773 & 0.635 & $0.621^{A}$ & $0.624^{c}$ \\
\hline & $1 / 2[3 / 2]^{o}$ & ${ }^{3} D^{o}$ & 2 & 307691.03 & $304226.90^{A}$ & $307398.42^{a}$ & 1.266 & 0.790 & $0.673^{A}$ & $0.766^{c}$ \\
\hline & $1 / 2[5 / 2]^{o}$ & ${ }^{1} F^{o}$ & 3 & 307972.21 & - & $308151.35^{a}$ & 1.119 & 0.794 & - & $0.775^{c}$ \\
\hline & $1 / 2[3 / 2]^{o}$ & ${ }^{3} D^{o}$ & 1 & 309874.94 & $293186.53^{A}$ & $310049.76^{a}$ & 0.832 & 0.104 & $0.055^{A}$ & $0.053^{c}$ \\
\hline \multirow[t]{7}{*}{$5 p^{5} 7 s$} & $3 / 2[3 / 2]^{o}$ & ${ }^{3} P^{o}$ & 2 & 292072.45 & $286514.39^{A}$ & $292080.31^{a}$ & 1.500 & 0.701 & $0.629^{A}$ & $0.737^{f}$ \\
\hline & $3 / 2[3 / 2]^{o}$ & ${ }^{1} P^{o}$ & 1 & 292785.70 & $285545.25^{A}$ & $292777.84^{a}$ & 1.176 & 0.296 & $0.186^{A}$ & $0.255^{c}$ \\
\hline & & & & & & & & & & $0.326^{f}$ \\
\hline & $1 / 2[1 / 2]^{o}$ & ${ }^{3} P^{o}$ & 0 & 313527.58 & $307977.61^{A}$ & $313512.37^{a}$ & 0.000 & 0.707 & $0.628^{A}$ & $0.672^{c}$ \\
\hline & & & & & & & & & & $0.729^{f}$ \\
\hline & $1 / 2[1 / 2]^{o}$ & ${ }^{3} P^{o}$ & 1 & 313901.71 & $307490.60^{A}$ & $313917.03^{a}$ & 1.322 & 0.357 & $0.240^{A}$ & $0.322^{c}$ \\
\hline & & & & & & & & & & $0.393^{f}$ \\
\hline \multirow[t]{3}{*}{$5 p^{5} 5 f$} & $3 / 2[3 / 2]$ & ${ }^{3} D$ & 1 & 293516.30 & - & $293509.80^{a}$ & 0.499 & 0.164 & - & $0.182^{c}$ \\
\hline & $3 / 2[3 / 2]$ & ${ }^{3} D$ & 2 & 294042.40 & - & $294027.80^{a}$ & 1.118 & 0.181 & - & $0.188^{c}$ \\
\hline & $3 / 2[9 / 2]$ & ${ }^{3} G$ & 5 & 294483.12 & - & $294517.20^{a}$ & 1.200 & 0.177 & - & $0.189^{c}$ \\
\hline
\end{tabular}


TABLE II (cont.)

\begin{tabular}{|c|c|c|c|c|c|c|c|c|c|c|}
\hline \multirow{2}{*}{ Conf. } & \multicolumn{2}{|c|}{ Term } & \multirow{2}{*}{$\mathrm{J}$} & \multicolumn{3}{|c|}{$\mathrm{E}\left[\mathrm{cm}^{-1}\right]$} & \multirow{2}{*}{$\frac{g \text {-factor }}{\text { HFR }}$} & \multicolumn{3}{|c|}{$\tau$ T [ns] } \\
\hline & $\mathrm{jK}$ & LS & & HFR & $\mathrm{MCDF}$ & Other w. & & HFR & MCDF & Other w. \\
\hline \multirow[t]{9}{*}{$5 p^{5} 5 f$} & $3 / 2[9 / 2]$ & ${ }^{1} G$ & 4 & 294562.71 & - & $294565.70^{a}$ & 1.026 & 0.178 & - & $0.181^{c}$ \\
\hline & $3 / 2[5 / 2]$ & ${ }^{3} D$ & 3 & 295448.71 & - & $295437.00^{a}$ & 1.202 & 0.181 & - & $0.187^{c}$ \\
\hline & $3 / 2[5 / 2]$ & ${ }^{1} D$ & 2 & 295889.18 & - & $295890.40^{a}$ & 0.856 & 0.213 & - & $0.182^{c}$ \\
\hline & $3 / 2[7 / 2]$ & ${ }^{1} F$ & 3 & 296338.54 & - & $296338.50^{a}$ & 0.947 & 0.182 & - & $0.179^{c}$ \\
\hline & $3 / 2[7 / 2]$ & ${ }^{3} F$ & 4 & 296555.82 & - & $296550.60^{a}$ & 1.181 & 0.193 & - & $0.187^{c}$ \\
\hline & $1 / 2[7 / 2]$ & ${ }^{3} G$ & 3 & 316122.71 & - & $316124.60^{a}$ & 0.836 & 0.172 & - & $0.177^{c}$ \\
\hline & $1 / 2[7 / 2]$ & ${ }^{3} G$ & 4 & 316434.58 & - & $316432.80^{a}$ & 1.094 & 0.186 & - & $0.186^{c}$ \\
\hline & $1 / 2[5 / 2]$ & ${ }^{3} F$ & 3 & 316472.01 & - & $316464.70^{a}$ & 1.185 & 0.183 & - & $0.187^{c}$ \\
\hline & $1 / 2[5 / 2]$ & ${ }^{3} F$ & 2 & 316666.96 & - & $316674.10^{a}$ & 0.861 & 0.194 & - & $0.176^{c}$ \\
\hline \multirow[t]{10}{*}{$5 p^{5} 7 p$} & $3 / 2[1 / 2]$ & ${ }^{3} S$ & 1 & 306533.49 & $303643.74^{A}$ & $306533.10^{a}$ & 1.807 & 1.096 & $1.129^{A}$ & $0.736^{c}$ \\
\hline & $3 / 2[5 / 2]$ & ${ }^{1} D$ & 2 & 307314.35 & - & $307314.30^{a}$ & 1.132 & 1.205 & - & $1.097^{c}$ \\
\hline & $3 / 2[5 / 2]$ & ${ }^{3} D$ & 3 & 308426.50 & $307012.82^{A}$ & $308427.20^{a}$ & 1.331 & 1.006 & $1.152^{A}$ & $0.820^{c}$ \\
\hline & $3 / 2[3 / 2]$ & ${ }^{1} P$ & 1 & 309041.77 & $308902.34^{A}$ & $309041.90^{a}$ & 1.059 & 1.275 & $1.356^{A}$ & $1.059^{c}$ \\
\hline & $3 / 2[3 / 2]$ & ${ }^{3} P$ & 2 & 309667.08 & $309874.57^{A}$ & $309666.90^{a}$ & 1.363 & 1.132 & $1.241^{A}$ & $1.129^{c}$ \\
\hline & $3 / 2[1 / 2]$ & ${ }^{1} S$ & 0 & 311556.41 & $336963.28^{A}$ & $311556.20^{a}$ & 0.000 & 1.527 & $0.671^{A}$ & $0.977^{c}$ \\
\hline & $1 / 2[3 / 2]$ & ${ }^{3} D$ & 1 & 328163.91 & $327925.73^{A}$ & $328164.10^{a}$ & 0.645 & 1.064 & $1.207^{A}$ & $0.933^{c}$ \\
\hline & $1 / 2[3 / 2]$ & ${ }^{3} D$ & 2 & 329916.91 & $329985.51^{A}$ & $329917.10^{a}$ & 1.169 & 1.003 & $1.238^{A}$ & $0.873^{c}$ \\
\hline & $1 / 2[1 / 2]$ & ${ }^{3} P$ & 1 & 329942.11 & $330155.80^{A}$ & $329941.70^{a}$ & 1.487 & 1.080 & $1.116^{A}$ & $1.005^{c}$ \\
\hline & $1 / 2[1 / 2]$ & ${ }^{3} P$ & 0 & 330318.67 & $317173.16^{A}$ & $330318.70^{a}$ & 0.000 & 1.393 & $0.967^{A}$ & $0.938^{c}$ \\
\hline \multirow[t]{4}{*}{$5 p^{5} 8 s$} & $3 / 2[3 / 2]^{\circ}$ & ${ }^{3} P^{o}$ & 2 & 333010.37 & $324225.63^{A}$ & $333008.70^{a}$ & 1.500 & 0.968 & $4.718^{A}$ & $0.908^{c}$ \\
\hline & $3 / 2[3 / 2]^{o}$ & ${ }^{1} P^{o}$ & 1 & 333298.71 & $324649.74^{A}$ & $333299.40^{a}$ & 1.157 & 0.270 & $0.944^{A}$ & $0.486^{c}$ \\
\hline & $1 / 2[1 / 2]^{o}$ & ${ }^{3} P^{o}$ & 0 & 354202.15 & $346299.02^{A}$ & $354205.30^{a}$ & 0.000 & 0.966 & $4.488^{A}$ & $0.905^{c}$ \\
\hline & $1 / 2[1 / 2]^{o}$ & ${ }^{3} P^{o}$ & 1 & 354289.79 & $346498.91^{A}$ & $354285.80^{a}$ & 1.304 & 0.893 & $2.218^{A}$ & $0.591^{c}$ \\
\hline
\end{tabular}

${ }^{a}$ Ref. [1], ${ }^{b}$ Ref. [2] — XIH(B), ${ }^{c}$ Ref. [3], ${ }^{d}$ Ref. [4] — Model B, ${ }^{e}$ Ref. [4] - MCDF, ${ }^{f}$ Ref. [5]. 
TABLE III

The wavelengths $\lambda$, logarithmic weighted oscillator strengths $\log (g f)$, and transition probabilities $A_{j i}$, for electric dipole (E1) transitions in La IV. Numbers in brackets represent powers of 10.

\begin{tabular}{|c|c|c|c|c|c|c|c|c|c|c|c|c|}
\hline \multicolumn{4}{|c|}{ Transitions } & \multicolumn{3}{|c|}{$\lambda[\mathrm{nm}]$} & \multicolumn{3}{|c|}{$\log (g f)$} & \multicolumn{3}{|c|}{$A_{j i}\left[\mathrm{~s}^{-1}\right]$} \\
\hline \multicolumn{2}{|c|}{ Upper levels } & \multicolumn{2}{|c|}{ Lower levels } & \multirow{2}{*}{ HFR } & \multirow{2}{*}{ MCDF } & \multirow{2}{*}{ Other w. } & \multirow{2}{*}{ HFR } & \multirow{2}{*}{ MCDF } & \multirow{2}{*}{ Other w. } & \multirow{2}{*}{ HFR } & \multirow{2}{*}{$\mathrm{MCDF}$} & \multirow{2}{*}{ Other w. } \\
\hline Conf. & Term & Conf. & Term & & & & & & & & & \\
\hline $5 p^{5} 6 s$ & ${ }^{1} P_{1}^{o}$ & $5 p^{6}$ & ${ }^{1} S_{0}$ & 49.958 & 50.348 & $\begin{array}{l}49.949^{a} \\
49.954^{b} \\
49.967^{e}\end{array}$ & -0.271 & -0.197 & $-0.288^{e}$ & $4.77(9)$ & $5.57(9)$ & $\begin{array}{l}3.79(9)^{a} \\
3.99(9)^{c} \\
4.65(9)^{d} \\
4.58(9)^{e}\end{array}$ \\
\hline $5 p^{5} 6 s$ & ${ }^{3} P_{1}^{o}$ & $5 p^{6}$ & ${ }^{1} S_{0}$ & 45.345 & 45.679 & $\begin{array}{l}45.356^{a} \\
45.350^{b} \\
45.335^{e}\end{array}$ & -0.148 & -0.223 & $-0.126^{e}$ & $7.70(9)$ & $6.37(9)$ & $\begin{array}{c}3.40(9)^{a} \\
1.05(10)^{c} \\
5.44(9)^{d} \\
8.09(9)^{e}\end{array}$ \\
\hline $5 p^{5} 5 d$ & ${ }^{1} P_{1}^{o}$ & $5 p^{6}$ & ${ }^{1} S_{0}$ & 46.310 & 43.668 & $\begin{array}{l}46.314^{a} \\
46.314^{b} \\
46.310^{e}\end{array}$ & 0.853 & 0.800 & $0.812^{e}$ & $7.40(10)$ & $7.36(10)$ & $\begin{array}{l}8.67(10)^{a} \\
5.04(10)^{c} \\
6.76(10)^{d} \\
6.72(10)^{e}\end{array}$ \\
\hline $5 p^{5} 5 d$ & ${ }^{3} D_{1}^{o}$ & $5 p^{6}$ & ${ }^{1} S_{0}$ & 55.296 & 53.784 & $\begin{array}{l}55.204^{a} \\
55.202^{b} \\
55.293^{e}\end{array}$ & -0.394 & -0.584 & $-0.442^{e}$ & $2.93(9)$ & $1.99(9)$ & $\begin{array}{l}3.65(9)^{a} \\
2.05(9)^{c} \\
1.64(9)^{d} \\
2.63(9)^{e}\end{array}$ \\
\hline $5 p^{5} 5 d$ & ${ }^{3} P_{1}^{o}$ & $5 p^{6}$ & ${ }^{1} S_{0}$ & 63.142 & 63.059 & $\begin{array}{l}63.122^{a} \\
63.126^{b} \\
63.163^{e}\end{array}$ & -2.362 & -2.239 & $-2.486^{e}$ & $2.43(7)$ & $3.22(7)$ & $\begin{array}{l}4.10(7)^{a} \\
1.69(7)^{c} \\
2.92(7)^{d} \\
1.82(7)^{e}\end{array}$ \\
\hline $5 p^{5} 6 p$ & ${ }^{3} S_{1}$ & $5 p^{5} 5 d$ & ${ }^{1} D_{2}^{o}$ & 159.108 & - & $\begin{array}{l}158.456^{a} \\
158.511^{b} \\
159.118^{e}\end{array}$ & -1.390 & - & $-1.511^{e}$ & $3.58(7)$ & - & $\begin{array}{l}4.06(7)^{a} \\
2.71(7)^{e}\end{array}$ \\
\hline $5 p^{5} 6 p$ & ${ }^{3} S_{1}$ & $5 p^{5} 5 d$ & ${ }^{3} P_{0}^{o}$ & 133.396 & 142.59 & $\begin{array}{l}133.525^{a} \\
133.496^{b} \\
133.322^{e}\end{array}$ & -0.614 & -0.599 & $-0.637^{e}$ & $3.04(8)$ & $2.74(8)$ & $\begin{array}{l}2.93(8)^{a} \\
2.89(8)^{e}\end{array}$ \\
\hline $5 p^{5} 6 p$ & ${ }^{3} S_{1}$ & $5 p^{5} 5 d$ & ${ }^{3} P_{1}^{o}$ & 137.591 & 147.81 & $\begin{array}{l}137.760^{a} \\
137.749^{b} \\
137.495^{e}\end{array}$ & -0.300 & -0.248 & $-0.306^{e}$ & $5.88(8)$ & $5.74(8)$ & $\begin{array}{l}5.87(8)^{a} \\
5.82(8)^{e}\end{array}$ \\
\hline $5 p^{5} 6 p$ & ${ }^{3} S_{1}$ & $5 p^{5} 5 d$ & ${ }^{3} P_{2}^{o}$ & 146.613 & 159.25 & $\begin{array}{l}146.656^{a} \\
146.754^{b} \\
146.463^{e}\end{array}$ & -0.314 & -0.224 & $-0.265^{e}$ & $5.02(8)$ & $5.23(8)$ & $\begin{array}{l}3.54(8)^{a} \\
5.63(8)^{e}\end{array}$ \\
\hline $5 p^{5} 6 p$ & ${ }^{3} S_{1}$ & $5 p^{5} 6 s$ & ${ }^{3} P_{2}^{o}$ & 305.518 & 338.43 & $\begin{array}{l}305.536^{a} \\
305.668^{b} \\
305.896^{e}\end{array}$ & 0.066 & 0.0005 & $0.051^{e}$ & $2.77(8)$ & $1.94(8)$ & $\begin{array}{l}3.13(8)^{a} \\
2.67(8)^{e}\end{array}$ \\
\hline $5 p^{5} 7 s$ & ${ }^{3} P_{2}^{o}$ & $5 p^{5} 6 p$ & ${ }^{3} S_{1}$ & 163.877 & 169.30 & $\begin{array}{l}163.776^{a} \\
163.742^{b} \\
162.914^{e}\end{array}$ & -0.257 & -0.288 & $-0.258^{e}$ & $2.75(8)$ & $2.39(8)$ & $\begin{array}{l}3.35(8)^{a} \\
2.78(8)^{e}\end{array}$ \\
\hline $5 p^{5} 6 p$ & ${ }^{3} D_{2}$ & $5 p^{5} 5 d$ & ${ }^{1} F_{3}^{o}$ & 235.478 & 282.02 & $\begin{array}{l}234.814^{a} \\
234.836^{b} \\
235.092^{e}\end{array}$ & -1.516 & -1.297 & $-1.479^{e}$ & $7.33(6)$ & $8.44(6)$ & $\begin{array}{l}3.95(6)^{a} \\
8.02(6)^{e}\end{array}$ \\
\hline $5 p^{5} 6 p$ & ${ }^{3} D_{2}$ & $5 p^{5} 5 d$ & ${ }^{3} D_{3}^{o}$ & 167.012 & 191.25 & $\begin{array}{l}166.575^{a} \\
166.484^{b} \\
166.530^{e}\end{array}$ & -1.126 & -0.898 & $-1.077^{e}$ & $3.58(7)$ & $4.60(7)$ & $\begin{array}{l}3.09(7)^{a} \\
4.03(7)^{e}\end{array}$ \\
\hline $5 p^{5} 6 p$ & ${ }^{3} D_{2}$ & $5 p^{5} 5 d$ & ${ }^{3} D_{2}^{o}$ & 222.739 & 264.22 & $\begin{array}{l}222.225^{a} \\
222.112^{b} \\
221.653^{e}\end{array}$ & -1.305 & -0.934 & $-1.109^{e}$ & $1.33(7)$ & $2.22(7)$ & $\begin{array}{l}2.02(6)^{a} \\
2.11(7)^{e}\end{array}$ \\
\hline $5 p^{5} 6 p$ & ${ }^{3} D_{2}$ & $5 p^{5} 5 d$ & ${ }^{3} F_{3}^{o}$ & 146.363 & 160.85 & $\begin{array}{l}146.302^{a} \\
146.347^{b} \\
146.204^{e}\end{array}$ & 0.119 & 0.151 & $0.113^{e}$ & $8.19(8)$ & $7.30(8)$ & $\begin{array}{l}7.61(8)^{a} \\
8.09(8)^{e}\end{array}$ \\
\hline
\end{tabular}


TABLE III (cont.)

\begin{tabular}{|c|c|c|c|c|c|c|c|c|c|c|c|c|}
\hline \multicolumn{4}{|c|}{ Transitions } & \multicolumn{3}{|c|}{$\lambda[\mathrm{nm}]$} & \multicolumn{3}{|c|}{$\log (g f)$} & \multicolumn{3}{|c|}{$A_{j i}\left[\mathrm{~s}^{-1}\right]$} \\
\hline \multicolumn{2}{|c|}{ Upper levels } & \multicolumn{2}{|c|}{ Lower levels } & \multirow{2}{*}{ HFR } & \multirow{2}{*}{ MCDF } & \multirow{2}{*}{ Other w. } & \multirow{2}{*}{ HFR } & \multirow{2}{*}{$\mathrm{MCDF}$} & \multirow{2}{*}{ Other w. } & \multirow{2}{*}{ HFR } & \multirow{2}{*}{$\mathrm{MCDF}$} & \multirow{2}{*}{ Other w. } \\
\hline Conf. & Term & Conf. & Term & & & & & & & & & \\
\hline $5 p^{5} 6 p$ & ${ }^{3} D_{2}$ & $5 p^{5} 5 d$ & ${ }^{1} D_{2}^{o}$ & 153.654 & - & $\begin{array}{c}152.682^{a} \\
152.719^{b} \\
153.405\end{array}$ & -0.461 & - & $-0.444^{e}$ & $1.95(8)$ & - & $\begin{array}{l}1.87(8)^{a} \\
2.04(8)^{e}\end{array}$ \\
\hline $5 p^{5} 6 p$ & ${ }^{3} D_{2}$ & $5 p^{5} 5 d$ & ${ }^{3} P_{1}^{o}$ & 133.494 & 140.77 & $\begin{array}{l}133.374^{a} \\
133.353^{b} \\
133.209^{e}\end{array}$ & -0.907 & -0.956 & $-0.908^{e}$ & $9.27(7)$ & $7.44(7)$ & $\begin{array}{l}4.55(7)^{a} \\
9.29(7)^{e}\end{array}$ \\
\hline $5 p^{5} 6 p$ & ${ }^{3} D_{2}$ & $5 p^{5} 6 s$ & ${ }^{3} P_{2}^{o}$ & 286.025 & 303.67 & $\begin{array}{l}284.768^{a} \\
284.830^{b} \\
285.460^{e}\end{array}$ & -0.033 & -0.129 & $-0.055^{e}$ & $1.51(8)$ & $1.07(8)$ & $\begin{array}{l}1.68(8)^{a} \\
1.44(8)^{e}\end{array}$ \\
\hline $5 p^{5} 7 s$ & ${ }^{3} P_{2}^{o}$ & $5 p^{5} 6 p$ & ${ }^{3} D_{2}$ & 170.095 & 179.59 & $\begin{array}{l}168.392^{a} \\
168.417^{b} \\
169.388^{e}\end{array}$ & -0.243 & -0.283 & $-0.226^{e}$ & $2.64(8)$ & $2.15(8)$ & $\begin{array}{l}5.04(8)^{a} \\
4.60(8)^{e}\end{array}$ \\
\hline $5 p^{5} 6 p$ & ${ }^{3} D_{3}$ & $5 p^{5} 5 d$ & ${ }^{3} D_{3}^{o}$ & 157.950 & 181.76 & $\begin{array}{l}157.789^{a} \\
157.592^{b} \\
157.916^{e}\end{array}$ & -0.697 & -0.629 & $-0.665^{e}$ & $7.67(7)$ & $6.77(7)$ & $\begin{array}{l}7.92(7)^{a} \\
8.27(7)^{e}\end{array}$ \\
\hline $5 p^{5} 6 p$ & ${ }^{3} D_{3}$ & $5 p^{5} 5 d$ & ${ }^{3} F_{4}^{o}$ & 136.988 & 148.62 & $\begin{array}{l}136.926^{a} \\
136.804^{b} \\
137.416^{e}\end{array}$ & 0.341 & 0.364 & $0.325^{e}$ & $1.11(9)$ & $9.98(8)$ & $\begin{array}{l}1.07(9)^{a} \\
1.07(9)^{e}\end{array}$ \\
\hline $5 p^{5} 6 p$ & ${ }^{3} D_{3}$ & $5 p^{5} 5 d$ & ${ }^{3} F_{3}^{o}$ & 139.356 & 154.08 & $\begin{array}{l}139.481^{a} \\
139.432^{b} \\
139.523^{e}\end{array}$ & -0.512 & -0.497 & $-0.515^{e}$ & $1.51(8)$ & $1.27(8)$ & $\begin{array}{l}1.43(8)^{a} \\
1.49(8)^{e}\end{array}$ \\
\hline $5 p^{5} 6 p$ & ${ }^{3} D_{3}$ & $5 p^{5} 5 d$ & ${ }^{3} P_{2}^{o}$ & 135.368 & 145.12 & $\begin{array}{l}135.288^{a} \\
135.276^{b} \\
135.332^{e}\end{array}$ & -0.638 & -0.656 & $-0.579^{e}$ & $1.20(8)$ & $9.98(7)$ & $\begin{array}{l}2.96(7)^{a} \\
1.37(8)^{e}\end{array}$ \\
\hline $5 p^{5} 7 s$ & ${ }^{3} P_{2}^{o}$ & $5 p^{5} 6 p$ & ${ }^{3} D_{3}$ & 180.650 & 194.44 & $\begin{array}{l}180.736^{a} \\
180.866^{b} \\
179.320^{e}\end{array}$ & 0.157 & -0.798 & $0.166^{e}$ & $5.87(8)$ & $2.40(7)$ & $\begin{array}{l}5.96(8)^{a} \\
6.08(8)^{e}\end{array}$ \\
\hline $5 p^{5} 6 p$ & ${ }^{1} P_{1}$ & $5 p^{5} 5 d$ & ${ }^{3} D_{1}^{o}$ & 175.727 & 208.57 & $\begin{array}{l}176.683^{a} \\
176.765^{b} \\
175.749^{e}\end{array}$ & -0.646 & -0.587 & $-0.591^{e}$ & $1.63(8)$ & $1.32(8)$ & $\begin{array}{l}1.12(8)^{a} \\
1.85(8)^{e}\end{array}$ \\
\hline $5 p^{5} 6 p$ & ${ }^{1} P_{1}$ & $5 p^{5} 5 d$ & ${ }^{1} D_{2}^{o}$ & 143.782 & - & $\begin{array}{l}143.186^{a} \\
143.255^{b} \\
143.785^{e}\end{array}$ & -0.123 & - & $-0.121^{e}$ & $8.11(8)$ & - & $\begin{array}{l}7.41(8)^{a} \\
8.14(8)^{e}\end{array}$ \\
\hline $5 p^{5} 6 p$ & ${ }^{1} P_{1}$ & $5 p^{5} 5 d$ & ${ }^{3} F_{2}^{o}$ & 189.631 & 160.20 & $\begin{array}{l}189.147^{a} \\
189.147^{b} \\
189.855^{e}\end{array}$ & -1.733 & -0.112 & $-1.700^{e}$ & $1.14(7)$ & $6.70(8)$ & $\begin{array}{l}5.76(6)^{a} \\
1.23(7)^{e}\end{array}$ \\
\hline $5 p^{5} 6 p$ & ${ }^{1} P_{1}$ & $5 p^{5} 5 d$ & ${ }^{3} P_{1}^{o}$ & 125.979 & 132.82 & $\begin{array}{l}126.071^{a} \\
126.079^{b} \\
125.894^{e}\end{array}$ & -1.625 & -2.213 & $-1.634^{e}$ & $3.32(7)$ & $7.71(6)$ & $\begin{array}{l}4.35(6)^{a} \\
3.26(7)^{e}\end{array}$ \\
\hline $5 p^{5} 6 p$ & ${ }^{1} P_{1}$ & $5 p^{5} 5 d$ & ${ }^{3} P_{2}^{o}$ & 133.501 & 141.98 & $\begin{array}{l}133.481^{a} \\
133.496^{b} \\
133.372^{e}\end{array}$ & -0.917 & -0.944 & $-0.986^{e}$ & $1.51(8)$ & $1.25(8)$ & $\begin{array}{l}1.88(8)^{a} \\
1.29(8)^{e}\end{array}$ \\
\hline $5 p^{5} 6 p$ & ${ }^{1} P_{1}$ & $5 p^{5} 6 s$ & ${ }^{3} P_{2}^{o}$ & 253.611 & 268.93 & $\begin{array}{l}253.422^{a} \\
253.275^{b} \\
253.853^{e}\end{array}$ & -2.308 & -1.468 & $-2.033^{e}$ & $1.70(6)$ & $1.04(7)$ & $\begin{array}{l}3.38(6)^{a} \\
3.20(6)^{e}\end{array}$ \\
\hline $5 p^{5} 6 p$ & ${ }^{3} P_{2}$ & $5 p^{5} 5 d$ & ${ }^{3} D_{3}^{o}$ & 150.792 & 171.51 & $\begin{array}{l}150.949^{a} \\
150.787^{b} \\
150.822^{e}\end{array}$ & 0.144 & 0.161 & $0.148^{e}$ & $8.18(8)$ & $6.60(8)$ & $\begin{array}{l}7.04(8)^{a} \\
8.25(8)^{e}\end{array}$ \\
\hline $5 p^{5} 6 p$ & ${ }^{3} P_{2}$ & $5 p^{5} 5 d$ & ${ }^{3} D_{2}^{o}$ & 194.795 & 227.98 & $\begin{array}{l}195.259^{a} \\
195.080^{b} \\
194.667^{e}\end{array}$ & -0.832 & -0.414 & $-0.655^{e}$ & $5.18(7)$ & $9.89(7)$ & $\begin{array}{l}1.18(7)^{a} \\
7.79(7)^{e}\end{array}$ \\
\hline
\end{tabular}


TABLE III (cont.)

\begin{tabular}{|c|c|c|c|c|c|c|c|c|c|c|c|c|}
\hline \multicolumn{4}{|c|}{ Transitions } & \multicolumn{3}{|c|}{$\lambda[\mathrm{nm}]$} & \multicolumn{3}{|c|}{$\log (g f)$} & \multicolumn{3}{|c|}{$A_{j i}\left[\mathrm{~s}^{-1}\right]$} \\
\hline \multicolumn{2}{|c|}{ Upper levels } & \multicolumn{2}{|c|}{ Lower levels } & \multirow{2}{*}{ HFR } & \multirow{2}{*}{$\mathrm{MCDF}$} & \multirow{2}{*}{ Other w. } & \multirow{2}{*}{ HFR } & \multirow{2}{*}{ MCDF } & \multirow{2}{*}{ Other w. } & \multirow{2}{*}{ HFR } & \multirow{2}{*}{ MCDF } & \multirow{2}{*}{ Other w } \\
\hline Conf. & Term & Conf. & Term & & & & & & & & & \\
\hline $5 p^{5} 6 p$ & ${ }^{3} P_{2}$ & $5 p^{5} 5 d$ & ${ }^{1} D_{2}^{o}$ & 139.820 & - & $\begin{array}{l}139.450^{a} \\
139.432^{b} \\
139.976^{e}\end{array}$ & -3.188 & - & $-3.792^{e}$ & $4.43(5)$ & - & $\begin{array}{l}2.64(5)^{a} \\
1.10(5)^{e}\end{array}$ \\
\hline $5 p^{5} 6 p$ & ${ }^{3} P_{2}$ & $5 p^{5} 5 d$ & ${ }^{3} P_{1}^{o}$ & 122.925 & 129.78 & $\begin{array}{l}123.166^{a} \\
123.090^{b} \\
122.965^{e}\end{array}$ & -1.079 & -1.062 & $-1.161^{e}$ & $7.37(7)$ & $6.86(7)$ & $\begin{array}{l}1.01(8)^{a} \\
6.09(7)^{e}\end{array}$ \\
\hline $5 p^{5} 6 p$ & ${ }^{3} P_{2}$ & $5 p^{5} 5 d$ & ${ }^{3} P_{2}^{o}$ & 130.076 & 138.51 & $\begin{array}{l}130.228^{a} \\
130.231^{b} \\
130.088^{e}\end{array}$ & -0.458 & -0.478 & $-0.536^{e}$ & $2.75(8)$ & $2.30(8)$ & $\begin{array}{l}3.60(8)^{a} \\
2.29(8)^{e}\end{array}$ \\
\hline $5 p^{5} 6 p$ & ${ }^{3} P_{2}$ & $5 p^{5} 6 s$ & ${ }^{1} P_{1}^{o}$ & 252.814 & 270.12 & $\begin{array}{l}253.732^{a} \\
253.576^{b} \\
253.088^{e}\end{array}$ & 0.040 & -0.024 & $0.030^{e}$ & $2.29(8)$ & $1.72(8)$ & $\begin{array}{l}2.36(8)^{a} \\
2.23(8)^{e}\end{array}$ \\
\hline $5 p^{5} 6 p$ & ${ }^{3} P_{2}$ & $5 p^{5} 6 s$ & ${ }^{3} P_{2}^{o}$ & 241.532 & 256.76 & $\begin{array}{l}241.950^{a} \\
241.758^{b} \\
242.217^{e}\end{array}$ & 0.147 & 0.115 & $0.143^{e}$ & $3.21(8)$ & $2.63(8)$ & $\begin{array}{l}1.68(8)^{a} \\
3.16(8)^{e}\end{array}$ \\
\hline $5 p^{5} 7 s$ & ${ }^{1} P_{1}^{o}$ & $5 p^{5} 6 p$ & ${ }^{3} P_{2}$ & 188.453 & 197.48 & $\begin{array}{l}188.073^{a} \\
188.157^{b} \\
189.459^{e}\end{array}$ & -0.299 & -0.201 & $-0.309^{e}$ & $3.14(8)$ & $3.59(8)$ & $\begin{array}{l}2.68(8)^{a} \\
3.04(8)^{e}\end{array}$ \\
\hline $5 p^{5} 7 s$ & ${ }^{3} P_{2}^{o}$ & $5 p^{5} 6 p$ & ${ }^{3} P_{2}$ & 191.021 & 201.34 & $\begin{array}{l}190.631^{a} \\
190.744^{b} \\
189.439^{e}\end{array}$ & -0.099 & 0.008 & $-0.069^{e}$ & $2.91(8)$ & $3.97(8)$ & $\begin{array}{l}2.08(8)^{a} \\
3.17(8)^{e}\end{array}$ \\
\hline $5 p^{5} 6 p$ & ${ }^{3} P_{0}$ & $5 p^{5} 6 s$ & ${ }^{1} P_{1}^{o}$ & 226.276 & 233.48 & $\begin{array}{l}226.382^{a} \\
226.591^{b} \\
226.024^{e}\end{array}$ & -0.256 & -0.265 & $-0.243^{e}$ & $7.23(8)$ & $6.64(8)$ & $\begin{array}{l}7.67(8)^{a} \\
7.47(8)^{e}\end{array}$ \\
\hline $5 p^{5} 7 s$ & ${ }^{1} P_{1}^{o}$ & $5 p^{5} 6 p$ & ${ }^{3} P_{0}$ & 206.507 & 223.07 & $\begin{array}{l}206.572^{a} \\
206.650^{b} \\
208.114^{e}\end{array}$ & -0.718 & -0.479 & $-0.713^{e}$ & $9.98(7)$ & $1.48(8)$ & $\begin{array}{l}9.08(7)^{a} \\
9.94(7)^{e}\end{array}$ \\
\hline $5 p^{5} 6 p$ & ${ }^{3} D_{1}$ & $5 p^{5} 5 d$ & ${ }^{1} P_{1}^{o}$ & 260.199 & 457.22 & $\begin{array}{l}260.583^{a} \\
260.801^{b} \\
260.889^{e}\end{array}$ & -0.742 & -0.854 & $-0.647^{e}$ & $5.94(7)$ & $1.49(7)$ & $\begin{array}{l}2.24(7)^{a} \\
7.36(7)^{e}\end{array}$ \\
\hline $5 p^{5} 6 p$ & ${ }^{3} D_{1}$ & $5 p^{5} 5 d$ & ${ }^{3} D_{1}^{o}$ & 136.005 & 153.98 & $\begin{array}{l}136.705^{a} \\
136.804^{b} \\
136.213^{e}\end{array}$ & -1.324 & -1.173 & $-1.296^{e}$ & $5.70(7)$ & $6.28(7)$ & $\begin{array}{l}6.11(7)^{a} \\
6.05(7)^{e}\end{array}$ \\
\hline $5 p^{5} 6 p$ & ${ }^{3} D_{1}$ & $5 p^{5} 5 d$ & ${ }^{1} D_{2}^{o}$ & 116.050 & - & $\begin{array}{l}115.753^{a} \\
115.835^{b} \\
116.193^{e}\end{array}$ & -1.506 & - & $-1.629^{e}$ & $5.15(7)$ & - & $\begin{array}{l}4.03(7)^{a} \\
3.87(7)^{e}\end{array}$ \\
\hline $5 p^{5} 6 p$ & ${ }^{3} D_{1}$ & $5 p^{5} 5 d$ & ${ }^{3} F_{2}^{o}$ & 144.187 & 125.91 & $\begin{array}{l}144.112^{a} \\
144.163^{b} \\
144.536^{e}\end{array}$ & -0.015 & -1.204 & $-0.021^{e}$ & $1.03(9)$ & $8.75(7)$ & $\begin{array}{l}9.83(8)^{a} \\
1.02(9)^{e}\end{array}$ \\
\hline $5 p^{5} 6 p$ & ${ }^{3} D_{1}$ & $5 p^{5} 6 s$ & ${ }^{3} P_{0}^{o}$ & 285.249 & 301.17 & $\begin{array}{l}286.286^{a} \\
286.330^{b} \\
285.691^{e}\end{array}$ & -0.306 & -0.309 & $-0.307^{e}$ & $1.35(8)$ & $1.20(8)$ & $\begin{array}{l}1.44(8)^{a} \\
1.34(8)^{e}\end{array}$ \\
\hline $5 p^{5} 7 s$ & ${ }^{1} P_{1}^{o}$ & $5 p^{5} 6 p$ & ${ }^{3} D_{1}$ & 260.314 & 282.47 & $\begin{array}{l}259.808^{a} \\
259.750^{b} \\
262.059^{e}\end{array}$ & -2.414 & -2.105 & $-2.420^{e}$ & $1.27(6)$ & $2.18(6)$ & $\begin{array}{l}8.19(5)^{a} \\
1.23(6)^{e}\end{array}$ \\
\hline $5 p^{5} 6 p$ & ${ }^{3} P_{1}$ & $5 p^{5} 5 d$ & ${ }^{3} D_{1}^{o}$ & 127.198 & 144.49 & $\begin{array}{l}128.654^{a} \\
128.319^{b} \\
127.799^{e}\end{array}$ & -1.286 & -1.211 & $-1.289^{e}$ & $7.12(7)$ & $6.55(7)$ & $\begin{array}{l}5.79(7)^{a} \\
7.00(7)^{e}\end{array}$ \\
\hline $5 p^{5} 6 p$ & ${ }^{3} P_{1}$ & $5 p^{5} 5 d$ & ${ }^{3} D_{2}^{o}$ & 140.696 & 157.82 & $\begin{array}{l}141.535^{a} \\
141.458^{b} \\
141.192^{e}\end{array}$ & -0.021 & -0.073 & $-0.041^{e}$ & $1.07(9)$ & $7.53(8)$ & $\begin{array}{l}1.08(9)^{a} \\
1.02(9)^{e}\end{array}$ \\
\hline
\end{tabular}


TABLE III (cont.)

\begin{tabular}{|c|c|c|c|c|c|c|c|c|c|c|c|c|}
\hline \multicolumn{4}{|c|}{ Transitions } & \multicolumn{3}{|c|}{$\lambda[\mathrm{nm}]$} & \multicolumn{3}{|c|}{$\log (g f)$} & \multicolumn{3}{|c|}{$A_{j i}\left[\mathrm{~s}^{-1}\right]$} \\
\hline \multicolumn{2}{|c|}{ Upper levels } & \multicolumn{2}{|c|}{ Lower levels } & \multirow{2}{*}{ HFR } & \multirow{2}{*}{ MCDF } & \multirow{2}{*}{ Other w. } & \multirow{2}{*}{ HFR } & \multirow{2}{*}{ MCDF } & \multirow{2}{*}{ Other w. } & \multirow{2}{*}{ HFR } & \multirow{2}{*}{ MCDF } & \multirow{2}{*}{ Other w. } \\
\hline Conf. & Term & Conf. & Term & & & & & & & & & \\
\hline $5 p^{5} 6 p$ & ${ }^{3} P_{1}$ & $5 p^{5} 5 d$ & ${ }^{3} P_{2}^{o}$ & 103.501 & 109.06 & $\begin{array}{l}103.920^{a} \\
103.930^{b} \\
103.813^{e}\end{array}$ & -1.372 & -1.264 & $-1.511^{e}$ & $8.81(7)$ & $1.01(8)$ & $\begin{array}{l}1.07(8)^{a} \\
6.35(7)^{e}\end{array}$ \\
\hline $5 p^{5} 6 p$ & ${ }^{3} P_{1}$ & $5 p^{5} 6 s$ & ${ }^{3} P_{2}^{o}$ & 163.555 & 171.09 & $\begin{array}{l}164.553^{a} \\
164.521^{b} \\
164.633^{e}\end{array}$ & -1.043 & -0.638 & $-0.905^{e}$ & $7.54(7)$ & $1.74(8)$ & $\begin{array}{l}3.52(7)^{a} \\
1.02(8)^{e}\end{array}$ \\
\hline $5 p^{5} 6 p$ & ${ }^{3} P_{1}$ & $5 p^{5} 6 s$ & ${ }^{3} P_{0}^{o}$ & 249.078 & 266.89 & $\begin{array}{l}251.959^{a} \\
251.502^{b} \\
251.027^{e}\end{array}$ & -0.038 & -0.062 & $-0.042^{e}$ & $3.28(8)$ & $2.70(8)$ & $\begin{array}{l}3.55(8)^{a} \\
3.21(8)^{e}\end{array}$ \\
\hline $5 p^{5} 6 p$ & ${ }^{3} P_{1}$ & $5 p^{5} 6 s$ & ${ }^{3} P_{1}^{o}$ & 256.866 & 276.11 & $\begin{array}{l}259.217^{a} \\
259.130^{b} \\
259.599^{e}\end{array}$ & -0.296 & -0.321 & $-0.317^{e}$ & $1.70(8)$ & $1.39(8)$ & $\begin{array}{l}1.90(8)^{a} \\
1.59(8)^{e}\end{array}$ \\
\hline $5 p^{5} 7 s$ & ${ }^{1} P_{1}^{o}$ & $5 p^{5} 6 p$ & ${ }^{3} P_{1}$ & 300.080 & 321.15 & $\begin{array}{l}296.464^{a} \\
296.258^{b} \\
300.067^{e}\end{array}$ & -3.464 & -2.807 & $-3.425^{e}$ & $8.48(4)$ & $3.36(5)$ & $\begin{array}{l}1.63(5)^{a} \\
9.27(4)^{e}\end{array}$ \\
\hline $5 p^{5} 6 p$ & ${ }^{1} D_{2}$ & $5 p^{5} 5 d$ & ${ }^{1} F_{3}^{o}$ & 145.358 & 163.53 & $\begin{array}{l}146.099^{a} \\
146.215^{b} \\
146.225^{e}\end{array}$ & 0.224 & 0.242 & $0.223^{e}$ & $1.06(9)$ & $8.71(8)$ & $\begin{array}{l}9.39(8)^{a} \\
1.04(9)^{e}\end{array}$ \\
\hline $5 p^{5} 6 p$ & ${ }^{1} D_{2}$ & $5 p^{5} 5 d$ & ${ }^{3} D_{3}^{o}$ & 116.000 & 128.24 & $\begin{array}{l}116.424^{a} \\
116.429^{b} \\
116.414^{e}\end{array}$ & -3.343 & -3.395 & $-3.158^{e}$ & $4.50(5)$ & $3.26(5)$ & $\begin{array}{l}5.37(5)^{a} \\
6.84(5)^{e}\end{array}$ \\
\hline $5 p^{5} 6 p$ & ${ }^{1} D_{2}$ & $5 p^{5} 5 d$ & ${ }^{3} F_{3}^{o}$ & 105.650 & 113.81 & $\begin{array}{l}106.144^{a} \\
106.209^{b} \\
106.103^{e}\end{array}$ & -1.363 & -1.207 & $-1.483^{e}$ & $5.18(7)$ & $6.39(7)$ & $\begin{array}{l}5.88(7)^{a} \\
3.89(7)^{e}\end{array}$ \\
\hline $5 p^{5} 6 p$ & ${ }^{1} S_{0}$ & $5 p^{5} 5 d$ & ${ }^{1} P_{1}^{o}$ & 224.902 & 341.06 & $\begin{array}{l}224.859^{a} \\
224.495^{b} \\
224.949^{e}\end{array}$ & -0.589 & -0.602 & $-0.478^{e}$ & $3.40(8)$ & $1.43(8)$ & $\begin{array}{l}2.52(8)^{a} \\
4.38(8)^{e}\end{array}$ \\
\hline $5 p^{5} 6 p$ & ${ }^{1} S_{0}$ & $5 p^{5} 5 d$ & ${ }^{3} D_{1}^{o}$ & 125.694 & 138.13 & $\begin{array}{l}126.188^{a} \\
126.112^{b} \\
125.725^{e}\end{array}$ & -1.133 & -1.060 & $-1.214^{e}$ & $3.11(8)$ & $3.04(8)$ & $\begin{array}{l}3.09(8)^{a} \\
2.58(8)^{e}\end{array}$ \\
\hline $5 p^{5} 6 p$ & ${ }^{1} S_{0}$ & $5 p^{5} 5 d$ & ${ }^{3} P_{1}^{o}$ & 98.010 & 100.26 & $\begin{array}{l}98.069^{a} \\
98.003^{b} \\
97.971^{e}\end{array}$ & -2.259 & -2.097 & $-2.507^{e}$ & $3.83(7)$ & $5.30(7)$ & $\begin{array}{l}8.46(7)^{a} \\
2.16(7)^{e}\end{array}$ \\
\hline $5 p^{5} 6 p$ & ${ }^{1} S_{0}$ & $5 p^{5} 6 s$ & ${ }^{3} P_{1}^{o}$ & 250.805 & 253.79 & $\begin{array}{l}250.530^{a} \\
250.281^{b} \\
251.183^{e}\end{array}$ & -0.259 & -0.265 & $-0.232^{e}$ & $5.84(8)$ & $5.61(8)$ & $\begin{array}{l}5.65(8)^{a} \\
6.20(8)^{e}\end{array}$ \\
\hline
\end{tabular}

${ }^{a}$ Ref. [5], ${ }^{b}$ Ref. [6], ${ }^{c}$ Ref. [4] - HFR $+\mathrm{CP}(\mathrm{B}),{ }^{d}$ Ref. [4] - MCDF, ${ }^{e}$ Ref. [7]. 
TABLE IV

New data (wavelengths $\lambda$, logarithmic weighted oscillator strengths $\log (g f)$, and transition probabilities $A_{j i}$ ) for electric dipole (E1) transitions in La IV. Numbers in brackets represent powers of 10.

\begin{tabular}{|c|c|c|c|c|c|c|c|c|c|c|c|}
\hline \multicolumn{3}{|c|}{ Upper level } & \multicolumn{3}{|c|}{ Lower level } & \multicolumn{2}{|c|}{$\lambda[\AA \AA]$} & \multicolumn{2}{|c|}{$\log (g f)$} & \multicolumn{2}{|c|}{$A_{j i}\left[\mathrm{~s}^{-1}\right]$} \\
\hline Conf. & Term & $J$ & Conf. & Term & $J$ & HFR & $\mathrm{MCDF}$ & HFR & MCDF & HFR & MCDF \\
\hline $5 p^{5} 7 s$ & ${ }^{1} P^{o}$ & 1 & $5 p^{6}$ & ${ }^{1} S$ & 0 & 341.56 & 349.32 & -0.983 & -0.684 & $1.984(9)$ & $3.77(9)$ \\
\hline $5 p^{5} 7 s$ & ${ }^{3} P^{o}$ & 1 & $5 p^{6}$ & ${ }^{1} S$ & 0 & 318.57 & 325.28 & -1.194 & -0.911 & $1.402(9)$ & $2.58(9)$ \\
\hline $5 p^{5} 8 s$ & ${ }^{1} P^{o}$ & 1 & $5 p^{6}$ & ${ }^{1} S$ & 0 & 300.03 & 308.30 & -0.960 & -1.423 & $2.706(9)$ & $0.88(9)$ \\
\hline $5 p^{5} 8 s$ & ${ }^{3} P^{o}$ & 1 & $5 p^{6}$ & ${ }^{1} S$ & 0 & 282.25 & 289.10 & -2.290 & -2.033 & $1.431(8)$ & $2.47(8)$ \\
\hline $5 p^{5} 6 d$ & ${ }^{3} D^{o}$ & 1 & $5 p^{6}$ & ${ }^{1} S$ & 0 & 322.71 & 341.41 & -0.420 & -0.072 & $0.811(10)$ & $1.62(10)$ \\
\hline $5 p^{5} 6 d$ & ${ }^{3} P^{o}$ & 1 & $5 p^{6}$ & ${ }^{1} S$ & 0 & 350.94 & 358.01 & -1.975 & -2.016 & $1.912(8)$ & $1.67(8)$ \\
\hline $5 p^{5} 6 p$ & ${ }^{3} S$ & 1 & $5 p^{5} 5 d$ & ${ }^{3} P^{o}$ & 0 & 1333.96 & 1425.90 & -0.614 & -0.600 & $3.04(8)$ & $2.75(8)$ \\
\hline $5 p^{5} 6 p$ & ${ }^{1} P$ & 1 & $5 p^{5} 5 d$ & ${ }^{3} P^{o}$ & 0 & 1224.53 & 1285.90 & -2.599 & -1.966 & $3.73(6)$ & $1.45(7)$ \\
\hline $5 p^{5} 6 p$ & ${ }^{3} D$ & 1 & $5 p^{5} 5 d$ & ${ }^{3} P^{o}$ & 0 & 1017.46 & 1055.20 & -1.867 & -2.277 & $2.92(7)$ & $1.06(7)$ \\
\hline $5 p^{5} 6 p$ & ${ }^{3} S$ & 1 & $5 p^{5} 5 d$ & ${ }^{3} P^{o}$ & 1 & 1375.91 & 1478.10 & -0.300 & -0.248 & $5.88(8)$ & $5.75(8)$ \\
\hline $5 p^{5} 6 p$ & ${ }^{3} D$ & 2 & $5 p^{5} 5 d$ & ${ }^{3} P^{o}$ & 1 & 1334.94 & 1407.70 & -0.907 & -0.956 & $9.27(7)$ & $7.44(7)$ \\
\hline $5 p^{5} 6 p$ & ${ }^{1} P$ & 1 & $5 p^{5} 5 d$ & ${ }^{3} P^{o}$ & 1 & 1259.79 & 1328.20 & -1.625 & -2.213 & $3.32(7)$ & $7.71(6)$ \\
\hline $5 p^{5} 6 p$ & ${ }^{3} P$ & 2 & $5 p^{5} 5 d$ & ${ }^{3} P^{o}$ & 1 & 1229.25 & 1297.80 & -1.079 & -1.062 & $7.37(7)$ & $6.86(7)$ \\
\hline $5 p^{5} 6 p$ & ${ }^{3} P$ & 0 & $5 p^{5} 5 d$ & ${ }^{3} P^{o}$ & 1 & 1162.94 & 1206.80 & -2.041 & -1.843 & $4.49(7)$ & $6.58(7)$ \\
\hline $5 p^{5} 6 p$ & ${ }^{3} D$ & 1 & $5 p^{5} 5 d$ & ${ }^{3} P^{o}$ & 1 & 1041.68 & 1083.50 & -1.610 & -1.791 & $5.03(7)$ & $3.07(7)$ \\
\hline $5 p^{5} 6 p$ & ${ }^{3} P$ & 1 & $5 p^{5} 5 d$ & ${ }^{3} P^{o}$ & 1 & 989.22 & 1035.70 & -3.288 & -3.508 & $1.17(6)$ & $6.43(5)$ \\
\hline $5 p^{5} 6 p$ & ${ }^{3} D$ & 2 & $5 p^{5} 5 d$ & ${ }^{3} P^{o}$ & 1 & 987.76 & 1470.70 & -1.981 & -0.956 & $1.43(7)$ & $7.44(7)$ \\
\hline $5 p^{5} 6 p$ & ${ }^{1} S$ & 0 & $5 p^{5} 5 d$ & ${ }^{3} P^{o}$ & 1 & 980.10 & 1002.60 & -2.259 & -2.098 & $3.83(7)$ & $5.30(7)$ \\
\hline $5 p^{5} 6 p$ & ${ }^{3} S$ & 1 & $5 p^{5} 5 d$ & ${ }^{3} P^{o}$ & 2 & 1466.13 & 1592.50 & -0.314 & -0.224 & $5.02(8)$ & $5.24(8)$ \\
\hline $5 p^{5} 6 p$ & ${ }^{3} D$ & 2 & $5 p^{5} 5 d$ & ${ }^{3} P^{o}$ & 2 & 1419.70 & 1511.10 & -1.442 & -1.848 & $2.39(7)$ & $8.30(6)$ \\
\hline $5 p^{5} 6 p$ & ${ }^{3} D$ & 3 & $5 p^{5} 5 d$ & ${ }^{3} P^{o}$ & 2 & 1353.68 & 1451.20 & -0.638 & -0.656 & $1.20(8)$ & $9.99(7)$ \\
\hline $5 p^{5} 6 p$ & ${ }^{1} P$ & 1 & $5 p^{5} 5 d$ & ${ }^{3} P^{o}$ & 2 & 1335.01 & 1419.80 & -0.917 & -0.944 & $1.51(8)$ & $1.26(8)$ \\
\hline $5 p^{5} 6 p$ & ${ }^{3} P$ & 2 & $5 p^{5} 5 d$ & ${ }^{3} P^{o}$ & 2 & 1300.76 & 1385.10 & -0.458 & -0.479 & $2.75(8)$ & $2.31(8)$ \\
\hline $5 p^{5} 6 p$ & ${ }^{3} D$ & 1 & $5 p^{5} 5 d$ & ${ }^{3} P^{o}$ & 2 & 1092.58 & 1143.80 & -2.628 & -3.204 & $4.38(6)$ & $1.06(6)$ \\
\hline $5 p^{5} 6 p$ & ${ }^{3} P$ & 1 & $5 p^{5} 5 d$ & ${ }^{3} P^{o}$ & 2 & 1035.01 & 1090.60 & -1.372 & -1.264 & $8.81(7)$ & $1.02(8)$ \\
\hline $5 p^{5} 6 p$ & ${ }^{3} D$ & 2 & $5 p^{5} 5 d$ & ${ }^{3} P^{o}$ & 2 & 1033.42 & 1511.70 & -1.341 & -1.848 & $5.69(7)$ & $8.30(6)$ \\
\hline $5 p^{5} 6 p$ & ${ }^{3} D$ & 3 & $5 p^{5} 5 d$ & ${ }^{3} F^{o}$ & 4 & 1369.88 & 1486.20 & 0.341 & 0.364 & $1.11(9)$ & $0.99(9)$ \\
\hline $5 p^{5} 6 p$ & ${ }^{3} D$ & 2 & $5 p^{5} 5 d$ & ${ }^{3} F^{o}$ & 3 & 1463.63 & 1608.50 & 0.119 & 0.151 & $8.19(8)$ & $7.30(8)$ \\
\hline $5 p^{5} 6 p$ & ${ }^{3} D$ & 3 & $5 p^{5} 5 d$ & ${ }^{3} F^{o}$ & 3 & 1393.56 & 1540.80 & -0.512 & -0.497 & $1.51(8)$ & $1.28(8)$ \\
\hline $5 p^{5} 6 p$ & ${ }^{3} P$ & 2 & $5 p^{5} 5 d$ & ${ }^{3} F^{o}$ & 3 & 1337.55 & 1466.50 & -2.663 & -1.857 & $1.62(6)$ & $8.63(6)$ \\
\hline $5 p^{5} 6 p$ & ${ }^{3} S$ & 1 & $5 p^{5} 5 d$ & ${ }^{3} F^{o}$ & 2 & 1591.08 & 1825.30 & -1.390 & -1.543 & $3.58(7)$ & $1.91(7)$ \\
\hline $5 p^{5} 6 p$ & ${ }^{3} D$ & 2 & $5 p^{5} 5 d$ & ${ }^{3} F^{o}$ & 2 & 1536.54 & 1719.10 & -0.461 & -0.398 & $1.95(8)$ & $1.81(8)$ \\
\hline $5 p^{5} 6 p$ & ${ }^{3} D$ & 3 & $5 p^{5} 5 d$ & ${ }^{3} F^{o}$ & 2 & 1459.51 & 1642.10 & -2.244 & -1.916 & $2.55(6)$ & $4.29(6)$ \\
\hline $5 p^{5} 6 p$ & ${ }^{1} P$ & 1 & $5 p^{5} 5 d$ & ${ }^{3} F^{o}$ & 2 & 1437.82 & 1602.00 & -0.123 & -0.112 & $8.11(8)$ & $6.70(8)$ \\
\hline $5 p^{5} 6 p$ & ${ }^{3} D$ & 1 & $5 p^{5} 5 d$ & ${ }^{3} F^{o}$ & 2 & 1160.50 & 1259.10 & -1.506 & -1.205 & $5.15(7)$ & $8.75(7)$ \\
\hline $5 p^{5} 6 p$ & ${ }^{3} P$ & 1 & $5 p^{5} 5 d$ & ${ }^{3} F^{o}$ & 2 & 1095.76 & 1195.00 & -3.021 & -2.466 & $1.76(6)$ & $5.33(6)$ \\
\hline $5 p^{5} 6 p$ & ${ }^{3} D$ & 2 & $5 p^{5} 5 d$ & ${ }^{3} D^{o}$ & 3 & 1670.12 & 1912.50 & -1.126 & -0.899 & $3.58(7)$ & $4.60(7)$ \\
\hline $5 p^{5} 6 p$ & ${ }^{3} D$ & 3 & $5 p^{5} 5 d$ & ${ }^{3} D^{o}$ & 3 & 1579.50 & 1817.60 & -0.697 & -0.629 & $7.67(7)$ & $6.78(7)$ \\
\hline $5 p^{5} 6 p$ & ${ }^{3} P$ & 2 & $5 p^{5} 5 d$ & ${ }^{3} D^{o}$ & 3 & 1507.92 & 1715.10 & 0.144 & 0.163 & $8.18(8)$ & $6.60(8)$ \\
\hline $5 p^{5} 6 p$ & ${ }^{3} S$ & 1 & $5 p^{5} 5 d$ & ${ }^{3} D^{o}$ & 1 & 1991.74 & 2480.80 & -1.797 & -1.986 & $8.94(6)$ & $3.73(6)$ \\
\hline $5 p^{5} 6 p$ & ${ }^{3} D$ & 2 & $5 p^{5} 5 d$ & ${ }^{3} D^{o}$ & 1 & 1907.02 & 2288.70 & -1.919 & -1.981 & $4.42(6)$ & $2.66(6)$ \\
\hline $5 p^{5} 6 p$ & ${ }^{1} P$ & 1 & $5 p^{5} 5 d$ & ${ }^{3} D^{o}$ & 1 & 1757.27 & 2085.70 & -0.646 & -0.587 & $1.63(8)$ & $1.32(8)$ \\
\hline $5 p^{5} 6 p$ & ${ }^{3} P$ & 0 & $5 p^{5} 5 d$ & ${ }^{3} D^{o}$ & 1 & 1574.37 & 1801.20 & -0.554 & -0.578 & $7.52(8)$ & $5.44(8)$ \\
\hline $5 p^{5} 6 p$ & ${ }^{3} D$ & 1 & $5 p^{5} 5 d$ & ${ }^{3} D^{o}$ & 1 & 1360.05 & 1539.80 & -1.324 & -1.174 & $5.70(7)$ & $6.29(7)$ \\
\hline $5 p^{5} 6 p$ & ${ }^{3} P$ & 1 & $5 p^{5} 5 d$ & ${ }^{3} D^{o}$ & 1 & 1271.98 & 1444.90 & -1.286 & -1.211 & $7.12(7)$ & $6.55(7)$ \\
\hline $5 p^{5} 6 p$ & ${ }^{1} S$ & 0 & $5 p^{5} 5 d$ & ${ }^{3} D^{o}$ & 1 & 1256.94 & 1381.30 & -1.133 & -1.060 & $3.11(8)$ & $3.04(8)$ \\
\hline $5 p^{5} 6 p$ & ${ }^{3} D$ & 2 & $5 p^{5} 5 d$ & ${ }^{3} F^{o}$ & 2 & 1340.58 & 1719.10 & -0.811 & -0.398 & $1.15(8)$ & $1.81(8)$ \\
\hline $5 p^{5} 6 p$ & ${ }^{3} S$ & 1 & $5 p^{5} 5 d$ & ${ }^{3} D^{o}$ & 2 & 2343.84 & 2901.50 & -1.998 & -1.355 & $4.07(6)$ & $1.17(7)$ \\
\hline $5 p^{5} 6 p$ & ${ }^{3} D$ & 2 & $5 p^{5} 5 d$ & ${ }^{3} D^{o}$ & 2 & 2227.39 & 2642.20 & -1.305 & -0.934 & $1.33(7)$ & $2.23(7)$ \\
\hline $5 p^{5} 6 p$ & ${ }^{3} D$ & 3 & $5 p^{5} 5 d$ & ${ }^{3} D^{o}$ & 2 & 2069.08 & 2464.50 & -1.333 & -0.732 & $1.03(7)$ & $2.91(7)$ \\
\hline
\end{tabular}


TABLE IV (cont.)

\begin{tabular}{|c|c|c|c|c|c|c|c|c|c|c|c|}
\hline \multicolumn{3}{|c|}{ Upper level } & \multicolumn{3}{|c|}{ Lower level } & \multicolumn{2}{|c|}{$\lambda[\AA]$} & \multicolumn{2}{|c|}{$\log (g f)$} & \multicolumn{2}{|c|}{$A_{j i}\left[\mathrm{~s}^{-1}\right]$} \\
\hline Conf. & Term & $J$ & Conf. & Term & $J$ & HFR & MCDF & HFR & MCDF & HFR & MCDF \\
\hline $5 p^{5} 6 p$ & ${ }^{1} P$ & 1 & $5 p^{5} 5 d$ & ${ }^{3} D^{o}$ & 2 & 2025.76 & 2375.30 & -2.010 & -1.568 & $5.29(6)$ & $1.06(7)$ \\
\hline $5 p^{5} 6 p$ & ${ }^{3} D$ & 1 & $5 p^{5} 5 d$ & ${ }^{3} D^{o}$ & 2 & 1515.51 & 1692.10 & -2.516 & -2.189 & $2.95(6)$ & $5.03(6)$ \\
\hline $5 p^{5} 6 p$ & ${ }^{3} P$ & 2 & $5 p^{5} 5 d$ & ${ }^{3} D^{o}$ & 2 & 1947.95 & 2279.80 & -0.832 & -0.414 & $5.18(7)$ & $9.90(7)$ \\
\hline $5 p^{5} 6 p$ & ${ }^{3} P$ & 1 & $5 p^{5} 5 d$ & ${ }^{3} D^{o}$ & 2 & 1406.96 & 1578.20 & -0.021 & -0.074 & $1.07(9)$ & $7.54(8)$ \\
\hline $5 p^{5} 6 p$ & ${ }^{3} D$ & 2 & $5 p^{5} 5 d$ & ${ }^{3} D^{o}$ & 2 & 1404.01 & 2642.20 & -0.975 & -0.934 & $7.17(7)$ & $2.23(7)$ \\
\hline $5 p^{5} 6 p$ & ${ }^{3} D$ & 2 & $5 p^{5} 5 d$ & ${ }^{1} F^{o}$ & 3 & 2354.78 & 2820.20 & -1.516 & -1.298 & $7.33(6)$ & $8.45(6)$ \\
\hline $5 p^{5} 6 p$ & ${ }^{3} D$ & 3 & $5 p^{5} 5 d$ & ${ }^{1} F^{o}$ & 3 & 2178.56 & 2618.60 & -2.161 & -1.824 & $1.39(6)$ & $2.08(6)$ \\
\hline $5 p^{5} 6 p$ & ${ }^{1} P$ & 1 & $5 p^{5} 5 d$ & ${ }^{1} P^{o}$ & 1 & 4584.66 & 20522.00 & -1.128 & -1.480 & $7.88(6)$ & $1.75(5)$ \\
\hline $5 p^{5} 6 p$ & ${ }^{3} P$ & 0 & $5 p^{5} 5 d$ & ${ }^{1} P^{o}$ & 1 & 3518.29 & 8035.20 & -1.013 & -1.171 & $5.22(7)$ & $6.97(6)$ \\
\hline $5 p^{5} 6 p$ & ${ }^{3} D$ & 1 & $5 p^{5} 5 d$ & ${ }^{1} P^{o}$ & 1 & 2601.99 & 4572.20 & -0.742 & -0.852 & $5.94(7)$ & $1.49(7)$ \\
\hline $5 p^{5} 6 p$ & ${ }^{3} P$ & 1 & $5 p^{5} 5 d$ & ${ }^{1} P^{o}$ & 1 & 2297.63 & 3826.10 & -0.917 & -1.024 & $5.10(7)$ & $1.44(7)$ \\
\hline $5 p^{5} 6 p$ & ${ }^{1} S$ & 0 & $5 p^{5} 5 d$ & ${ }^{1} P^{o}$ & 1 & 2249.02 & 3410.60 & -0.589 & -0.602 & $3.40(8)$ & $1.43(8)$ \\
\hline $5 p^{5} 6 p$ & ${ }^{3} S$ & 1 & $5 p^{5} 6 s$ & ${ }^{3} P^{o}$ & 2 & 3055.18 & 3384.30 & 0.066 & 0.001 & $2.77(8)$ & $1.94(8)$ \\
\hline $5 p^{5} 6 p$ & ${ }^{3} D$ & 2 & $5 p^{5} 6 s$ & ${ }^{3} P^{o}$ & 2 & 2860.25 & 3036.70 & -0.033 & -0.130 & $1.51(8)$ & $1.07(8)$ \\
\hline $5 p^{5} 6 p$ & ${ }^{3} D$ & 3 & $5 p^{5} 6 s$ & ${ }^{3} P^{o}$ & 2 & 2604.36 & 2804.30 & 0.515 & 0.476 & $4.60(8)$ & $3.63(8)$ \\
\hline $5 p^{5} 6 p$ & ${ }^{1} P$ & 1 & $5 p^{5} 6 s$ & ${ }^{3} P^{o}$ & 2 & 2536.11 & 2689.30 & -2.308 & -1.468 & $1.70(6)$ & $1.05(7)$ \\
\hline $5 p^{5} 6 p$ & ${ }^{3} P$ & 2 & $5 p^{5} 6 s$ & ${ }^{3} P^{o}$ & 2 & 2415.32 & 2567.60 & 0.147 & 0.115 & $3.21(8)$ & $2.64(8)$ \\
\hline $5 p^{5} 6 p$ & ${ }^{3} D$ & 1 & $5 p^{5} 6 s$ & ${ }^{3} P^{o}$ & 2 & 1784.10 & 1845.60 & -2.737 & -2.438 & $1.28(6)$ & $2.38(6)$ \\
\hline $5 p^{5} 6 p$ & ${ }^{3} P$ & 1 & $5 p^{5} 6 s$ & ${ }^{3} P^{o}$ & 2 & 1635.55 & 1710.90 & -1.043 & -0.638 & $7.54(7)$ & $1.75(8)$ \\
\hline $5 p^{5} 6 p$ & ${ }^{3} D$ & 2 & $5 p^{5} 6 s$ & ${ }^{1} P^{o}$ & 1 & 3019.84 & 3225.40 & 0.071 & 0.099 & $1.72(8)$ & $1.61(8)$ \\
\hline $5 p^{5} 6 p$ & ${ }^{1} P$ & 1 & $5 p^{5} 6 s$ & ${ }^{1} P^{o}$ & 1 & 2660.79 & 2836.30 & 0.144 & 0.111 & $4.37(8)$ & $3.57(8)$ \\
\hline $5 p^{5} 6 p$ & ${ }^{3} P$ & 2 & $5 p^{5} 6 s$ & ${ }^{1} P^{o}$ & 1 & 2528.14 & 2701.20 & 0.040 & -0.025 & $2.29(8)$ & $1.73(8)$ \\
\hline $5 p^{5} 6 p$ & ${ }^{3} P$ & 0 & $5 p^{5} 6 s$ & ${ }^{1} P^{o}$ & 1 & 2262.76 & 2334.80 & -0.256 & -0.265 & $7.23(8)$ & $6.64(8)$ \\
\hline $5 p^{5} 6 p$ & ${ }^{3} D$ & 1 & $5 p^{5} 6 s$ & ${ }^{1} P^{o}$ & 1 & 1844.91 & 1913.70 & -2.343 & -2.252 & $2.96(6)$ & $3.40(6)$ \\
\hline $5 p^{5} 6 p$ & ${ }^{1} S$ & 0 & $5 p^{5} 6 s$ & ${ }^{1} P^{o}$ & 1 & 1660.17 & 1674.90 & -1.806 & -1.692 & $3.79(7)$ & $4.83(7)$ \\
\hline $5 p^{5} 6 p$ & ${ }^{3} D$ & 1 & $5 p^{5} 6 s$ & ${ }^{3} P^{o}$ & 0 & 2852.49 & 3011.70 & -0.306 & -0.310 & $1.35(8)$ & $1.20(8)$ \\
\hline $5 p^{5} 6 p$ & ${ }^{3} P$ & 1 & $5 p^{5} 6 s$ & ${ }^{3} P^{o}$ & 0 & 2490.78 & 2668.90 & -0.038 & -0.063 & $3.28(8)$ & $2.70(8)$ \\
\hline $5 p^{5} 6 p$ & ${ }^{3} P$ & 0 & $5 p^{5} 6 s$ & ${ }^{3} P^{o}$ & 1 & 4196.28 & 4439.10 & -2.509 & -2.131 & $1.17(6)$ & $2.50(6)$ \\
\hline $5 p^{5} 6 p$ & ${ }^{3} D$ & 1 & $5 p^{5} 6 s$ & ${ }^{3} P^{o}$ & 1 & 2955.09 & 3129.60 & -0.135 & -0.149 & $1.86(8)$ & $1.61(8)$ \\
\hline $5 p^{5} 6 p$ & ${ }^{3} P$ & 1 & $5 p^{5} 6 s$ & ${ }^{3} P^{o}$ & 1 & 2568.66 & 2761.10 & -0.296 & -0.322 & $1.70(8)$ & $1.39(8)$ \\
\hline $5 p^{5} 6 p$ & ${ }^{1} S$ & 0 & $5 p^{5} 6 s$ & ${ }^{3} P^{o}$ & 1 & 2508.05 & 2537.90 & -0.259 & -0.266 & $5.84(8)$ & $5.61(8)$ \\
\hline $5 p^{5} 6 d$ & ${ }^{3} P^{o}$ & 0 & $5 p^{5} 6 p$ & ${ }^{3} S$ & 1 & 1881.07 & 1927.30 & -0.065 & -0.080 & $1.62(9)$ & $1.49(9)$ \\
\hline $5 p^{5} 6 d$ & ${ }^{3} P^{o}$ & 0 & $5 p^{5} 6 p$ & ${ }^{1} P$ & 1 & 2152.30 & 2259.80 & -2.111 & -1.671 & $1.12(7)$ & $2.79(7)$ \\
\hline $5 p^{5} 6 d$ & ${ }^{3} P^{o}$ & 0 & $5 p^{5} 6 p$ & ${ }^{3} P$ & 1 & 4040.28 & 4350.10 & -2.033 & -1.676 & $3.79(6)$ & $7.43(6)$ \\
\hline $5 p^{5} 6 d$ & ${ }^{3} P^{o}$ & 1 & $5 p^{5} 6 p$ & ${ }^{3} S$ & 1 & 1855.42 & 1883.90 & 0.272 & 0.290 & $1.21(9)$ & $1.22(9)$ \\
\hline $5 p^{5} 6 d$ & ${ }^{3} P^{o}$ & 1 & $5 p^{5} 6 p$ & ${ }^{3} D$ & 2 & 1935.53 & 2012.10 & -0.508 & -0.630 & $1.84(8)$ & $1.29(8)$ \\
\hline $5 p^{5} 6 d$ & ${ }^{3} P^{o}$ & 1 & $5 p^{5} 6 p$ & ${ }^{1} P$ & 1 & 2118.78 & 2200.40 & -2.023 & -2.175 & $4.69(6)$ & $3.07(6)$ \\
\hline $5 p^{5} 6 d$ & ${ }^{3} P^{o}$ & 1 & $5 p^{5} 6 p$ & ${ }^{3} P$ & 2 & 2211.17 & 2289.30 & -0.465 & -0.420 & $1.56(8)$ & $1.61(8)$ \\
\hline $5 p^{5} 6 d$ & ${ }^{3} P^{o}$ & 1 & $5 p^{5} 6 p$ & ${ }^{3} P$ & 0 & 2463.91 & 2640.40 & -1.508 & -1.615 & $1.14(7)$ & $7.73(6)$ \\
\hline $5 p^{5} 6 d$ & ${ }^{3} P^{o}$ & 1 & $5 p^{5} 6 p$ & ${ }^{3} P$ & 1 & 3923.77 & 4135.20 & -1.634 & -1.646 & $3.36(6)$ & $2.94(6)$ \\
\hline $5 p^{5} 6 d$ & ${ }^{3} F^{o}$ & 4 & $5 p^{5} 6 p$ & ${ }^{3} D$ & 3 & 2036.73 & 2064.90 & 0.864 & 0.906 & $1.30(9)$ & $1.40(9)$ \\
\hline $5 p^{5} 6 d$ & ${ }^{3} F^{o}$ & 3 & $5 p^{5} 6 p$ & ${ }^{3} D$ & 2 & 1892.38 & 1926.80 & 0.719 & 0.726 & $1.39(9)$ & $1.36(9)$ \\
\hline $5 p^{5} 6 d$ & ${ }^{3} F^{o}$ & 3 & $5 p^{5} 6 p$ & ${ }^{3} D$ & 3 & 2023.94 & 2033.70 & -0.191 & -0.028 & $1.50(8)$ & $2.16(8)$ \\
\hline $5 p^{5} 6 d$ & ${ }^{3} F^{o}$ & 3 & $5 p^{5} 6 p$ & ${ }^{3} P$ & 2 & 2155.02 & 2179.40 & -0.810 & -2.023 & $3.18(7)$ & $1.90(6)$ \\
\hline $5 p^{5} 6 d$ & ${ }^{3} P^{o}$ & 2 & $5 p^{5} 6 p$ & ${ }^{3} S$ & 1 & 1815.15 & 1818.80 & 0.133 & 0.231 & $5.50(8)$ & $6.87(8)$ \\
\hline $5 p^{5} 6 d$ & ${ }^{3} P^{o}$ & 2 & $5 p^{5} 6 p$ & ${ }^{3} D$ & 2 & 1891.75 & 1938.10 & -0.583 & -0.676 & $9.73(7)$ & $7.49(7)$ \\
\hline $5 p^{5} 6 d$ & ${ }^{3} P^{o}$ & 2 & $5 p^{5} 6 p$ & ${ }^{3} D$ & 3 & 2023.23 & 2046.30 & -0.473 & -0.489 & $1.10(8)$ & $1.03(8)$ \\
\hline $5 p^{5} 6 d$ & ${ }^{3} P^{o}$ & 2 & $5 p^{5} 6 p$ & ${ }^{1} P$ & 1 & 2066.43 & 2112.20 & -0.287 & -0.394 & $1.61(8)$ & $1.21(8)$ \\
\hline $5 p^{5} 6 d$ & ${ }^{3} P^{o}$ & 2 & $5 p^{5} 6 p$ & ${ }^{3} P$ & 2 & 2154.21 & 2193.90 & 0.205 & 0.243 & $4.61(8)$ & $4.85(8)$ \\
\hline $5 p^{5} 6 d$ & ${ }^{3} P^{o}$ & 2 & $5 p^{5} 6 p$ & ${ }^{3} D$ & 1 & 3147.39 & 3295.30 & -2.002 & -2.086 & $1.34(6)$ & $1.01(6)$ \\
\hline $5 p^{5} 6 d$ & ${ }^{1} D^{o}$ & 2 & $5 p^{5} 6 p$ & ${ }^{3} S$ & 1 & 1776.04 & 1752.50 & -0.591 & -1.103 & $1.09(8)$ & $3.43(7)$ \\
\hline $5 p^{5} 6 d$ & ${ }^{1} D^{o}$ & 2 & $5 p^{5} 6 p$ & ${ }^{3} D$ & 2 & 1849.31 & 1862.90 & 0.137 & 0.180 & $5.35(8)$ & $5.82(8)$ \\
\hline
\end{tabular}


TABLE IV (cont.)

\begin{tabular}{|c|c|c|c|c|c|c|c|c|c|c|c|}
\hline \multicolumn{3}{|c|}{ Upper level } & \multicolumn{3}{|c|}{ Lower level } & \multicolumn{2}{|c|}{$\lambda[\AA]$} & \multicolumn{2}{|c|}{$\log (g f)$} & \multicolumn{2}{|c|}{$A_{j i}\left[\mathrm{~s}^{-1}\right]$} \\
\hline Conf. & Term & $J$ & Conf. & Term & $J$ & HFR & MCDF & HFR & MCDF & HFR & MCDF \\
\hline $5 p^{5} 6 d$ & ${ }^{1} D^{o}$ & 2 & $5 p^{5} 6 p$ & ${ }^{3} D$ & 3 & 1974.76 & 1962.70 & -1.330 & -1.323 & $1.60(7)$ & $1.65(7)$ \\
\hline $5 p^{5} 6 d$ & ${ }^{1} D^{o}$ & 2 & $5 p^{5} 6 p$ & ${ }^{1} P$ & 1 & 2015.89 & 2023.20 & 0.404 & 0.463 & $8.33(8)$ & $9.46(8)$ \\
\hline $5 p^{5} 6 d$ & ${ }^{3} D^{o}$ & 3 & $5 p^{5} 6 p$ & ${ }^{3} D$ & 3 & 1956.30 & 1921.10 & 0.060 & 0.080 & $2.86(8)$ & $3.10(8)$ \\
\hline $5 p^{5} 6 d$ & ${ }^{3} D^{o}$ & 3 & $5 p^{5} 6 p$ & ${ }^{3} P$ & 2 & 2078.49 & 2050.70 & 0.644 & 0.707 & $9.72(8)$ & $1.15(9)$ \\
\hline $5 p^{5} 6 d$ & ${ }^{1} P^{o}$ & 1 & $5 p^{5} 6 p$ & ${ }^{1} P$ & 1 & 1887.17 & 1228.60 & -0.081 & -0.962 & $5.18(8)$ & $1.61(8)$ \\
\hline $5 p^{5} 6 d$ & ${ }^{1} P^{o}$ & 1 & $5 p^{5} 6 p$ & ${ }^{3} P$ & 2 & 1960.11 & 1255.90 & -1.795 & -2.037 & $9.27(6)$ & $1.29(7)$ \\
\hline $5 p^{5} 6 d$ & ${ }^{1} P^{o}$ & 1 & $5 p^{5} 6 p$ & ${ }^{3} P$ & 0 & 2156.18 & 1354.70 & 0.108 & -1.072 & $6.13(8)$ & $1.03(8)$ \\
\hline $5 p^{5} 6 d$ & ${ }^{3} F^{o}$ & 2 & $5 p^{5} 6 p$ & ${ }^{1} P$ & 1 & 1439.09 & 1451.80 & -2.092 & -3.154 & $5.21(6)$ & $4.44(5)$ \\
\hline $5 p^{5} 6 d$ & ${ }^{3} F^{o}$ & 2 & $5 p^{5} 6 p$ & ${ }^{3} D$ & 1 & 1891.50 & 1927.50 & 0.563 & 0.577 & $1.36(9)$ & $1.35(9)$ \\
\hline $5 p^{5} 6 d$ & ${ }^{3} F^{o}$ & 2 & $5 p^{5} 6 p$ & ${ }^{3} P$ & 1 & 2093.05 & 2100.20 & -1.111 & -0.961 & $2.36(7)$ & $3.31(7)$ \\
\hline $5 p^{5} 6 d$ & ${ }^{3} F^{o}$ & 2 & $5 p^{5} 6 p$ & ${ }^{3} D$ & 2 & 2099.61 & 1367.40 & -0.268 & -2.272 & $1.63(8)$ & $3.82(6)$ \\
\hline $5 p^{5} 6 d$ & ${ }^{3} D^{o}$ & 1 & $5 p^{5} 6 p$ & ${ }^{3} S$ & 1 & 1268.65 & 1500.00 & -2.851 & -3.565 & $1.95(6)$ & $2.69(5)$ \\
\hline $5 p^{5} 6 d$ & ${ }^{3} D^{o}$ & 1 & $5 p^{5} 6 p$ & ${ }^{1} P$ & 1 & 1386.49 & 1694.00 & -2.952 & -0.051 & $1.29(6)$ & $6.90(8)$ \\
\hline $5 p^{5} 6 d$ & ${ }^{3} D^{o}$ & 1 & $5 p^{5} 6 p$ & ${ }^{3} P$ & 2 & 1425.46 & 1746.10 & -2.836 & -2.456 & $1.60(6)$ & $2.55(6)$ \\
\hline $5 p^{5} 6 d$ & ${ }^{3} D^{o}$ & 1 & $5 p^{5} 6 p$ & ${ }^{3} P$ & 0 & 1526.40 & 1943.20 & -1.447 & 0.181 & $3.41(7)$ & $8.92(8)$ \\
\hline $5 p^{5} 6 d$ & ${ }^{3} D^{o}$ & 1 & $5 p^{5} 6 p$ & ${ }^{3} D$ & 1 & 1801.65 & 2379.00 & -0.211 & -1.026 & $4.21(8)$ & $3.70(7)$ \\
\hline $5 p^{5} 6 d$ & ${ }^{3} D^{o}$ & 1 & $5 p^{5} 6 p$ & ${ }^{3} P$ & 1 & 1983.59 & 2647.60 & -0.452 & -1.096 & $2.00(8)$ & $2.54(7)$ \\
\hline $5 p^{5} 6 d$ & ${ }^{3} D^{o}$ & 1 & $5 p^{5} 6 p$ & ${ }^{3} D$ & 2 & 1989.48 & 1580.10 & -1.211 & -1.048 & $3.46(7)$ & $7.97(7)$ \\
\hline $5 p^{5} 6 d$ & ${ }^{3} D^{o}$ & 1 & $5 p^{5} 6 p$ & ${ }^{1} S$ & 0 & 2021.31 & 2891.40 & 0.131 & -1.442 & $7.36(8)$ & $9.61(6)$ \\
\hline $5 p^{5} 7 s$ & ${ }^{3} P^{o}$ & 2 & $5 p^{5} 6 p$ & ${ }^{3} S$ & 1 & 1638.77 & 1693.00 & -0.257 & -0.288 & $2.75(8)$ & $2.40(8)$ \\
\hline $5 p^{5} 7 s$ & ${ }^{3} P^{o}$ & 2 & $5 p^{5} 6 p$ & ${ }^{3} D$ & 2 & 1700.95 & 1795.90 & -0.243 & -0.284 & $2.64(8)$ & $2.15(8)$ \\
\hline $5 p^{5} 7 s$ & ${ }^{3} P^{o}$ & 2 & $5 p^{5} 6 p$ & ${ }^{3} D$ & 3 & 1806.50 & 1888.40 & 0.157 & 0.277 & $5.87(8)$ & $7.08(8)$ \\
\hline $5 p^{5} 7 s$ & ${ }^{3} P^{o}$ & 2 & $5 p^{5} 6 p$ & ${ }^{1} P$ & 1 & 1840.87 & 1944.40 & -1.733 & -1.166 & $7.28(6)$ & $2.41(7)$ \\
\hline $5 p^{5} 7 s$ & ${ }^{3} P^{o}$ & 2 & $5 p^{5} 6 p$ & ${ }^{3} P$ & 2 & 1910.21 & 2013.40 & -0.099 & 0.082 & $2.91(8)$ & $3.97(8)$ \\
\hline $5 p^{5} 7 s$ & ${ }^{3} P^{o}$ & 2 & $5 p^{5} 6 p$ & ${ }^{3} P$ & 1 & 3066.45 & 3314.90 & -1.959 & -1.640 & $1.56(6)$ & $2.78(6)$ \\
\hline $5 p^{5} 7 s$ & ${ }^{1} P^{o}$ & 1 & $5 p^{5} 6 p$ & ${ }^{3} S$ & 1 & 1619.84 & 1665.70 & -2.719 & -2.128 & $1.62(6)$ & $5.97(6)$ \\
\hline $5 p^{5} 7 s$ & ${ }^{1} P^{o}$ & 1 & $5 p^{5} 6 p$ & ${ }^{3} D$ & 2 & 1680.56 & 1765.10 & -0.230 & -0.138 & $4.64(8)$ & $5.19(8)$ \\
\hline $5 p^{5} 7 s$ & ${ }^{1} P^{o}$ & 1 & $5 p^{5} 6 p$ & ${ }^{1} P$ & 1 & 1817.01 & 1908.40 & -0.119 & -0.039 & $5.12(8)$ & $5.58(8)$ \\
\hline $5 p^{5} 7 s$ & ${ }^{1} P^{o}$ & 1 & $5 p^{5} 6 p$ & ${ }^{3} P$ & 2 & 1884.53 & 1974.80 & -0.299 & -0.201 & $3.14(8)$ & $3.59(8)$ \\
\hline $5 p^{5} 7 s$ & ${ }^{1} P^{o}$ & 1 & $5 p^{5} 6 p$ & ${ }^{3} P$ & 0 & 2065.07 & 2230.70 & -0.718 & -0.479 & $9.98(7)$ & $1.48(8)$ \\
\hline $5 p^{5} 7 s$ & ${ }^{1} P^{o}$ & 1 & $5 p^{5} 6 p$ & ${ }^{3} D$ & 1 & 2603.14 & 2824.70 & -2.414 & -2.105 & $1.27(6)$ & $2.19(6)$ \\
\hline $5 p^{5} 7 s$ & ${ }^{1} P^{o}$ & 1 & $5 p^{5} 6 p$ & ${ }^{1} S$ & 0 & 3087.99 & 3577.40 & -1.685 & -1.320 & $4.82(6)$ & $8.31(6)$ \\
\hline $5 p^{5} 7 s$ & ${ }^{3} P^{o}$ & 0 & $5 p^{5} 6 p$ & ${ }^{3} S$ & 1 & 1212.47 & 1239.10 & -3.035 & -2.505 & $4.19(6)$ & $1.36(7)$ \\
\hline $5 p^{5} 7 s$ & ${ }^{3} P^{o}$ & 0 & $5 p^{5} 6 p$ & ${ }^{3} D$ & 1 & 1690.42 & 1783.50 & -0.577 & -0.531 & $6.18(8)$ & $6.17(8)$ \\
\hline $5 p^{5} 7 s$ & ${ }^{3} P^{o}$ & 0 & $5 p^{5} 6 p$ & ${ }^{3} P$ & 1 & 1849.59 & 1930.40 & -0.393 & -0.272 & $7.88(8)$ & $9.58(8)$ \\
\hline $5 p^{5} 7 s$ & ${ }^{3} P^{o}$ & 1 & $5 p^{5} 6 p$ & ${ }^{3} S$ & 1 & 1206.99 & 1231.70 & -2.777 & -2.324 & $2.55(6)$ & $6.95(6)$ \\
\hline $5 p^{5} 7 s$ & ${ }^{3} P^{o}$ & 1 & $5 p^{5} 6 p$ & ${ }^{3} D$ & 2 & 1240.39 & 1285.20 & -3.156 & -3.316 & $1.01(6)$ & $6.50(5)$ \\
\hline $5 p^{5} 7 s$ & ${ }^{3} P^{o}$ & 1 & $5 p^{5} 6 p$ & ${ }^{1} P$ & 1 & 1313.17 & 1359.50 & -2.620 & -2.626 & $3.09(6)$ & $2.84(6)$ \\
\hline $5 p^{5} 7 s$ & ${ }^{3} P^{o}$ & 1 & $5 p^{5} 6 p$ & ${ }^{3} P$ & 0 & 1438.01 & 1515.60 & -2.040 & -2.142 & $9.82(6)$ & $6.98(6)$ \\
\hline $5 p^{5} 7 s$ & ${ }^{3} P^{o}$ & 1 & $5 p^{5} 6 p$ & ${ }^{3} D$ & 1 & 1679.79 & 1768.20 & -0.358 & -0.375 & $3.46(8)$ & $3.00(8)$ \\
\hline $5 p^{5} 7 s$ & ${ }^{3} P^{o}$ & 1 & $5 p^{5} 6 p$ & ${ }^{3} P$ & 1 & 1836.88 & 1912.40 & -0.604 & -0.534 & $1.64(8)$ & $1.78(8)$ \\
\hline $5 p^{5} 7 s$ & ${ }^{3} P^{o}$ & 1 & $5 p^{5} 6 p$ & ${ }^{1} S$ & 0 & 1869.18 & 2036.40 & -0.586 & -0.414 & $1.65(8)$ & $2.07(8)$ \\
\hline $5 p^{5} 8 s$ & ${ }^{3} P^{o}$ & 2 & $5 p^{5} 6 p$ & ${ }^{3} S$ & 1 & 980.78 & 1023.60 & -1.057 & -1.435 & $1.22(8)$ & $4.68(7)$ \\
\hline $5 p^{5} 8 s$ & ${ }^{3} P^{o}$ & 2 & $5 p^{5} 6 p$ & ${ }^{3} D$ & 2 & 1002.72 & 1060.30 & -1.103 & -1.707 & $1.05(8)$ & $2.33(7)$ \\
\hline $5 p^{5} 8 s$ & ${ }^{3} P^{o}$ & 2 & $5 p^{5} 6 p$ & ${ }^{3} D$ & 3 & 1038.49 & 1091.90 & -0.655 & -1.095 & $2.74(8)$ & $8.98(7)$ \\
\hline $5 p^{5} 8 s$ & ${ }^{3} P^{o}$ & 2 & $5 p^{5} 6 p$ & ${ }^{1} P$ & 1 & 1049.76 & 1110.30 & -2.845 & -3.310 & $1.73(6)$ & $5.30(5)$ \\
\hline $5 p^{5} 8 s$ & ${ }^{3} P^{o}$ & 2 & $5 p^{5} 6 p$ & ${ }^{3} P$ & 2 & 1071.95 & 1132.50 & -0.977 & -1.560 & $1.22(8)$ & $2.87(7)$ \\
\hline $5 p^{5} 8 s$ & ${ }^{3} P^{o}$ & 2 & $5 p^{5} 6 p$ & ${ }^{3} P$ & 1 & 1359.64 & 1453.50 & -2.743 & -3.617 & $1.30(6)$ & $1.52(5)$ \\
\hline $5 p^{5} 8 s$ & ${ }^{1} P^{o}$ & 1 & $5 p^{5} 6 p$ & ${ }^{3} D$ & 2 & 999.83 & 1055.50 & -1.003 & -1.488 & $2.21(8)$ & $6.49(7)$ \\
\hline $5 p^{5} 8 s$ & ${ }^{1} P^{o}$ & 1 & $5 p^{5} 6 p$ & ${ }^{1} P$ & 1 & 1046.59 & 1105.10 & -1.237 & -1.543 & $1.18(8)$ & $5.22(7)$ \\
\hline $5 p^{5} 8 s$ & ${ }^{1} P^{o}$ & 1 & $5 p^{5} 6 p$ & ${ }^{3} P$ & 2 & 1068.64 & 1127.10 & -1.120 & -1.718 & $1.48(8)$ & $3.35(7)$ \\
\hline $5 p^{5} 8 s$ & ${ }^{1} P^{o}$ & 1 & $5 p^{5} 6 p$ & ${ }^{3} P$ & 0 & 1124.39 & 1206.00 & -1.240 & -2.386 & $1.01(8)$ & $6.29(6)$ \\
\hline
\end{tabular}


TABLE IV (cont.)

\begin{tabular}{|c|c|c|c|c|c|c|c|c|c|c|c|}
\hline \multicolumn{3}{|c|}{ Upper level } & \multicolumn{3}{|c|}{ Lower level } & \multicolumn{2}{|c|}{$\lambda[\AA]$} & \multicolumn{2}{|c|}{$\log (g f)$} & \multicolumn{2}{|c|}{$A_{j i}\left[\mathrm{~s}^{-1}\right]$} \\
\hline Conf. & Term & $J$ & Conf. & Term & $J$ & HFR & MCDF & HFR & MCDF & HFR & MCDF \\
\hline $5 p^{5} 8 s$ & ${ }^{3} P^{o}$ & 0 & $5 p^{5} 6 p$ & ${ }^{3} S$ & 1 & 812.01 & 837.09 & -3.198 & -2.849 & $6.41(6)$ & $1.35(7)$ \\
\hline $5 p^{5} 8 s$ & ${ }^{3} P^{o}$ & 0 & $5 p^{5} 6 p$ & ${ }^{3} D$ & 1 & 1001.69 & 1054.60 & -1.412 & -1.900 & $2.57(8)$ & $7.56(7)$ \\
\hline $5 p^{5} 8 s$ & ${ }^{3} P^{o}$ & 0 & $5 p^{5} 6 p$ & ${ }^{3} P$ & 1 & 1055.51 & 1104.20 & -1.220 & -1.700 & $3.61(8)$ & $1.09(8)$ \\
\hline $5 p^{5} 8 s$ & ${ }^{3} P^{o}$ & 1 & $5 p^{5} 6 p$ & ${ }^{3} S$ & 1 & 811.43 & 835.69 & -3.067 & -2.735 & $2.89(6)$ & $5.86(6)$ \\
\hline $5 p^{5} 8 s$ & ${ }^{3} P^{o}$ & 1 & $5 p^{5} 6 p$ & ${ }^{3} D$ & 2 & 826.39 & 859.99 & -2.077 & -3.296 & $2.73(7)$ & $1.52(6)$ \\
\hline $5 p^{5} 8 s$ & ${ }^{3} P^{o}$ & 1 & $5 p^{5} 6 p$ & ${ }^{1} P$ & 1 & 858.08 & 892.64 & -2.054 & -3.445 & $2.67(7)$ & $1.00(6)$ \\
\hline $5 p^{5} 8 s$ & ${ }^{3} P^{o}$ & 1 & $5 p^{5} 6 p$ & ${ }^{3} P$ & 2 & 872.85 & 906.92 & -2.496 & -3.761 & $9.32(6)$ & $4.69(5)$ \\
\hline $5 p^{5} 8 s$ & ${ }^{3} P^{o}$ & 1 & $5 p^{5} 6 p$ & ${ }^{3} P$ & 0 & 909.68 & 957.36 & -2.161 & -2.665 & $1.85(7)$ & $5.25(6)$ \\
\hline $5 p^{5} 8 s$ & ${ }^{3} P^{o}$ & 1 & $5 p^{5} 6 p$ & ${ }^{3} D$ & 1 & 1000.81 & 1052.30 & -1.291 & -1.674 & $1.14(8)$ & $4.25(7)$ \\
\hline $5 p^{5} 8 s$ & ${ }^{3} P^{o}$ & 1 & $5 p^{5} 6 p$ & ${ }^{3} P$ & 1 & 1054.54 & 1101.80 & -1.521 & -1.887 & $6.02(7)$ & $2.38(7)$ \\
\hline $5 p^{5} 8 s$ & ${ }^{3} P^{o}$ & 1 & $5 p^{5} 6 p$ & ${ }^{1} S$ & 0 & 1065.10 & 1141.80 & -1.599 & -2.423 & $4.93(7)$ & $6.44(6)$ \\
\hline $5 p^{5} 7 p$ & ${ }^{3} S$ & 1 & $5 p^{5} 5 d$ & ${ }^{3} P^{o}$ & 0 & 664.69 & 678.64 & -1.611 & -1.729 & $1.23(8)$ & $9.01(7)$ \\
\hline $5 p^{5} 7 p$ & ${ }^{3} D$ & 1 & $5 p^{5} 5 d$ & ${ }^{3} P^{o}$ & 0 & 581.13 & 583.52 & -2.100 & -2.974 & $5.23(7)$ & $6.94(6)$ \\
\hline $5 p^{5} 7 p$ & ${ }^{3} P$ & 1 & $5 p^{5} 5 d$ & ${ }^{3} P^{o}$ & 0 & 575.19 & 576.75 & -2.832 & -4.058 & $9.89(6)$ & $5.85(5)$ \\
\hline $5 p^{5} 7 p$ & ${ }^{3} S$ & 1 & $5 p^{5} 5 d$ & ${ }^{3} P^{o}$ & 1 & 674.94 & 690.25 & -1.252 & -1.416 & $2.73(8)$ & $1.79(8)$ \\
\hline $5 p^{5} 7 p$ & ${ }^{3} D$ & 2 & $5 p^{5} 5 d$ & ${ }^{3} P^{o}$ & 1 & 671.40 & 676.96 & -2.239 & -1.967 & $1.71(7)$ & $3.14(7)$ \\
\hline $5 p^{5} 7 p$ & ${ }^{3} P$ & 2 & $5 p^{5} 5 d$ & ${ }^{3} P^{o}$ & 1 & 660.96 & 661.81 & -1.976 & -1.992 & $3.23(7)$ & $3.10(7)$ \\
\hline $5 p^{5} 7 p$ & ${ }^{1} S$ & 0 & $5 p^{5} 5 d$ & ${ }^{3} P^{o}$ & 1 & 652.81 & 561.27 & -2.957 & -2.618 & $1.73(7)$ & $5.10(7)$ \\
\hline $5 p^{5} 7 p$ & ${ }^{3} D$ & 1 & $5 p^{5} 5 d$ & ${ }^{3} P^{o}$ & 1 & 588.96 & 592.08 & -1.907 & -2.430 & $7.94(7)$ & $2.35(7)$ \\
\hline $5 p^{5} 7 p$ & ${ }^{3} P$ & 1 & $5 p^{5} 5 d$ & ${ }^{3} P^{o}$ & 1 & 582.85 & 585.11 & -3.589 & -4.444 & $1.69(6)$ & $2.34(5)$ \\
\hline $5 p^{5} 7 p$ & ${ }^{3} P$ & 0 & $5 p^{5} 5 d$ & ${ }^{3} P^{o}$ & 1 & 581.58 & 631.14 & -2.804 & -2.704 & $3.10(7)$ & $3.31(7)$ \\
\hline $5 p^{5} 7 p$ & ${ }^{3} S$ & 1 & $5 p^{5} 5 d$ & ${ }^{3} P^{o}$ & 2 & 695.95 & 714.21 & -1.413 & -1.528 & $1.78(8)$ & $1.29(8)$ \\
\hline $5 p^{5} 7 p$ & ${ }^{3} D$ & 2 & $5 p^{5} 5 d$ & ${ }^{3} P^{o}$ & 2 & 692.19 & 699.99 & -2.023 & -3.162 & $2.64(7)$ & $1.87(6)$ \\
\hline $5 p^{5} 7 p$ & ${ }^{3} D$ & 3 & $5 p^{5} 5 d$ & ${ }^{3} P^{o}$ & 2 & 686.90 & 697.50 & -1.957 & -1.676 & $2.23(7)$ & $4.13(7)$ \\
\hline $5 p^{5} 7 p$ & ${ }^{1} P$ & 1 & $5 p^{5} 5 d$ & ${ }^{3} P^{o}$ & 2 & 684.01 & 688.28 & -1.924 & -2.242 & $5.66(7)$ & $2.69(7)$ \\
\hline $5 p^{5} 7 p$ & ${ }^{3} P$ & 2 & $5 p^{5} 5 d$ & ${ }^{3} P^{o}$ & 2 & 681.09 & 683.80 & -1.294 & -1.496 & $1.46(8)$ & $9.11(7)$ \\
\hline $5 p^{5} 7 p$ & ${ }^{3} D$ & 1 & $5 p^{5} 5 d$ & ${ }^{3} P^{o}$ & 2 & 604.89 & 609.62 & -3.271 & -3.880 & $3.25(6)$ & $7.88(5)$ \\
\hline $5 p^{5} 7 p$ & ${ }^{3} P$ & 1 & $5 p^{5} 5 d$ & ${ }^{3} P^{o}$ & 2 & 598.45 & 602.24 & -1.945 & -1.972 & $7.04(7)$ & $6.54(7)$ \\
\hline $5 p^{5} 7 p$ & ${ }^{3} D$ & 3 & $5 p^{5} 5 d$ & ${ }^{3} F^{o}$ & 4 & 691.04 & 705.48 & -0.655 & -0.773 & $4.41(8)$ & $3.23(8)$ \\
\hline $5 p^{5} 7 p$ & ${ }^{3} D$ & 2 & $5 p^{5} 5 d$ & ${ }^{3} F^{o}$ & 3 & 702.46 & 720.19 & -0.890 & -1.126 & $3.49(8)$ & $1.92(8)$ \\
\hline $5 p^{5} 7 p$ & ${ }^{3} D$ & 3 & $5 p^{5} 5 d$ & ${ }^{3} F^{o}$ & 3 & 697.02 & 717.56 & -1.399 & -1.732 & $7.83(7)$ & $3.43(7)$ \\
\hline $5 p^{5} 7 p$ & ${ }^{3} P$ & 2 & $5 p^{5} 5 d$ & ${ }^{3} F^{o}$ & 3 & 691.04 & 703.06 & -2.885 & -2.603 & $3.64(6)$ & $6.74(6)$ \\
\hline $5 p^{5} 7 p$ & ${ }^{3} S$ & 1 & $5 p^{5} 5 d$ & ${ }^{3} F^{o}$ & 2 & 722.89 & 757.54 & -2.385 & -3.722 & $1.75(7)$ & $7.34(5)$ \\
\hline $5 p^{5} 7 p$ & ${ }^{3} D$ & 3 & $5 p^{5} 5 d$ & ${ }^{3} F^{o}$ & 2 & 713.14 & 738.77 & -2.824 & -3.387 & $2.81(6)$ & $7.16(5)$ \\
\hline $5 p^{5} 7 p$ & ${ }^{1} P$ & 1 & $5 p^{5} 5 d$ & ${ }^{3} F^{o}$ & 2 & 710.02 & 728.43 & -1.117 & -1.354 & $3.37(8)$ & $1.85(8)$ \\
\hline $5 p^{5} 7 p$ & ${ }^{3} D$ & 1 & $5 p^{5} 5 d$ & ${ }^{3} F^{o}$ & 2 & 625.14 & 640.92 & -2.127 & -2.029 & $4.24(7)$ & $5.06(7)$ \\
\hline $5 p^{5} 7 p$ & ${ }^{3} D$ & 2 & $5 p^{5} 5 d$ & ${ }^{3} D^{o}$ & 3 & 746.78 & 775.37 & -1.958 & -2.688 & $2.63(7)$ & $4.55(6)$ \\
\hline $5 p^{5} 7 p$ & ${ }^{3} D$ & 3 & $5 p^{5} 5 d$ & ${ }^{3} D^{o}$ & 3 & 740.63 & 772.32 & -1.510 & -2.211 & $5.37(7)$ & $9.84(6)$ \\
\hline $5 p^{5} 7 p$ & ${ }^{3} P$ & 2 & $5 p^{5} 5 d$ & ${ }^{3} D^{o}$ & 3 & 733.88 & 755.56 & -0.871 & -1.247 & $3.34(8)$ & $1.32(8)$ \\
\hline $5 p^{5} 7 p$ & ${ }^{3} S$ & 1 & $5 p^{5} 5 d$ & ${ }^{3} D^{o}$ & 1 & 795.61 & 850.85 & -2.599 & -3.683 & $8.83(6)$ & $6.38(5)$ \\
\hline $5 p^{5} 7 p$ & ${ }^{1} P$ & 1 & $5 p^{5} 5 d$ & ${ }^{3} D^{o}$ & 1 & 780.04 & 814.30 & -1.801 & -2.684 & $5.77(7)$ & $6.95(6)$ \\
\hline $5 p^{5} 7 p$ & ${ }^{3} P$ & 2 & $5 p^{5} 5 d$ & ${ }^{3} D^{o}$ & 1 & 776.26 & 808.04 & -2.980 & -3.468 & $2.32(6)$ & $6.95(5)$ \\
\hline $5 p^{5} 7 p$ & ${ }^{1} S$ & 0 & $5 p^{5} 5 d$ & ${ }^{3} D^{o}$ & 1 & 765.04 & 663.04 & -1.712 & -1.736 & $2.21(8)$ & $2.79(8)$ \\
\hline $5 p^{5} 7 p$ & ${ }^{3} D$ & 1 & $5 p^{5} 5 d$ & ${ }^{3} D^{o}$ & 1 & 678.79 & 706.46 & -2.343 & -2.295 & $2.19(7)$ & $2.26(7)$ \\
\hline $5 p^{5} 7 p$ & ${ }^{3} P$ & 1 & $5 p^{5} 5 d$ & ${ }^{3} D^{o}$ & 1 & 670.70 & 696.57 & -2.145 & -2.188 & $3.54(7)$ & $2.97(7)$ \\
\hline $5 p^{5} 7 p$ & ${ }^{3} P$ & 0 & $5 p^{5} 5 d$ & ${ }^{3} D^{o}$ & 1 & 669.01 & 762.78 & -1.747 & -2.356 & $2.67(8)$ & $5.05(7)$ \\
\hline $5 p^{5} 7 p$ & ${ }^{3} D$ & 2 & $5 p^{5} 5 d$ & ${ }^{3} F^{o}$ & 2 & 690.13 & 741.56 & -1.909 & -1.763 & $3.45(7)$ & $4.18(7)$ \\
\hline $5 p^{5} 7 p$ & ${ }^{3} P$ & 1 & $5 p^{5} 5 d$ & ${ }^{3} F^{o}$ & 2 & 690.01 & 632.76 & -2.511 & -3.545 & $1.44(7)$ & $1.58(6)$ \\
\hline $5 p^{5} 7 p$ & ${ }^{3} S$ & 1 & $5 p^{5} 5 d$ & ${ }^{3} D^{o}$ & 2 & 846.40 & 895.38 & -2.616 & -2.461 & $7.52(6)$ & $9.60(6)$ \\
\hline $5 p^{5} 7 p$ & ${ }^{3} D$ & 2 & $5 p^{5} 5 d$ & ${ }^{3} D^{o}$ & 2 & 840.84 & 873.14 & -2.479 & -4.167 & $6.26(6)$ & $1.19(5)$ \\
\hline $5 p^{5} 7 p$ & ${ }^{3} D$ & 3 & $5 p^{5} 5 d$ & ${ }^{3} D^{o}$ & 2 & 833.05 & 869.28 & -1.450 & -3.242 & $4.87(7)$ & $7.23(5)$ \\
\hline $5 p^{5} 7 p$ & ${ }^{1} P$ & 1 & $5 p^{5} 5 d$ & ${ }^{3} D^{o}$ & 2 & 828.80 & 855.00 & -2.741 & -3.745 & $5.87(6)$ & $5.48(5)$ \\
\hline
\end{tabular}


TABLE IV (cont.)

\begin{tabular}{|c|c|c|c|c|c|c|c|c|c|c|c|}
\hline \multicolumn{3}{|c|}{ Upper level } & \multicolumn{3}{|c|}{ Lower level } & \multicolumn{2}{|c|}{$\lambda[\AA]$} & \multicolumn{2}{|c|}{$\log (g f)$} & \multicolumn{2}{|c|}{$A_{j i}\left[\mathrm{~s}^{-1}\right]$} \\
\hline Conf. & Term & $J$ & Conf. & Term & $J$ & HFR & MCDF & HFR & MCDF & HFR & MCDF \\
\hline $5 p^{5} 7 p$ & ${ }^{3} P$ & 2 & $5 p^{5} 5 d$ & ${ }^{3} D^{o}$ & 2 & 824.53 & 848.10 & -2.314 & -2.467 & $9.51(6)$ & $6.33(6)$ \\
\hline $5 p^{5} 7 p$ & ${ }^{3} D$ & 1 & $5 p^{5} 5 d$ & ${ }^{3} D^{o}$ & 2 & 715.42 & 736.89 & -3.371 & -4.091 & $1.85(6)$ & $3.32(5)$ \\
\hline $5 p^{5} 7 p$ & ${ }^{3} P$ & 1 & $5 p^{5} 5 d$ & ${ }^{3} D^{o}$ & 2 & 706.43 & 726.13 & -1.028 & -1.335 & $4.18(8)$ & $1.95(8)$ \\
\hline $5 p^{5} 7 p$ & ${ }^{3} D$ & 2 & $5 p^{5} 5 d$ & ${ }^{1} P^{o}$ & 1 & 1094.38 & 1293.60 & -2.632 & -2.089 & $2.60(6)$ & $6.49(6)$ \\
\hline $5 p^{5} 7 p$ & ${ }^{1} S$ & 0 & $5 p^{5} 5 d$ & ${ }^{1} P^{o}$ & 1 & 1045.83 & 928.09 & -2.767 & -1.298 & $1.04(7)$ & $3.90(8)$ \\
\hline $5 p^{5} 7 p$ & ${ }^{3} D$ & 1 & $5 p^{5} 5 d$ & ${ }^{1} P^{o}$ & 1 & 891.06 & 1015.50 & -2.417 & -1.883 & $1.07(7)$ & $2.82(7)$ \\
\hline $5 p^{5} 7 p$ & ${ }^{3} P$ & 1 & $5 p^{5} 5 d$ & ${ }^{1} P^{o}$ & 1 & 877.16 & 995.14 & -2.526 & -2.221 & $8.60(6)$ & $1.35(7)$ \\
\hline $5 p^{5} 7 p$ & ${ }^{3} P$ & 0 & $5 p^{5} 5 d$ & ${ }^{1} P^{o}$ & 1 & 874.28 & 1136.00 & -1.934 & -1.294 & $1.02(8)$ & $2.63(8)$ \\
\hline $5 p^{5} 7 p$ & ${ }^{3} S$ & 1 & $5 p^{5} 6 s$ & ${ }^{3} P^{o}$ & 2 & 924.10 & 936.61 & -2.534 & -1.509 & $7.62(6)$ & $7.84(7)$ \\
\hline $5 p^{5} 7 p$ & ${ }^{3} D$ & 3 & $5 p^{5} 6 s$ & ${ }^{3} P^{o}$ & 2 & 908.21 & 908.09 & -2.267 & -2.217 & $6.25(6)$ & $7.00(6)$ \\
\hline $5 p^{5} 7 p$ & ${ }^{1} P$ & 1 & $5 p^{5} 6 s$ & ${ }^{3} P^{o}$ & 2 & 903.16 & 892.51 & -3.164 & -4.051 & $1.87(6)$ & $2.48(5)$ \\
\hline $5 p^{5} 7 p$ & ${ }^{3} D$ & 2 & $5 p^{5} 6 s$ & ${ }^{3} P^{o}$ & 2 & 759.89 & 912.31 & -2.810 & -2.255 & $3.58(6)$ & $8.90(6)$ \\
\hline $5 p^{5} 7 p$ & ${ }^{3} P$ & 1 & $5 p^{5} 6 s$ & ${ }^{3} P^{o}$ & 2 & 759.75 & 753.01 & -2.145 & -1.652 & $2.76(7)$ & $8.74(7)$ \\
\hline $5 p^{5} 7 p$ & ${ }^{3} S$ & 1 & $5 p^{5} 6 s$ & ${ }^{1} P^{o}$ & 1 & 940.15 & 953.82 & -2.782 & -2.462 & $4.15(6)$ & $8.44(6)$ \\
\hline $5 p^{5} 7 p$ & ${ }^{3} D$ & 2 & $5 p^{5} 6 s$ & ${ }^{1} P^{o}$ & 1 & 933.30 & 928.63 & -2.695 & -1.637 & $3.09(6)$ & $3.57(7)$ \\
\hline $5 p^{5} 7 p$ & ${ }^{1} P$ & 1 & $5 p^{5} 6 s$ & ${ }^{1} P^{o}$ & 1 & 918.49 & 908.13 & -3.220 & -2.876 & $1.59(6)$ & $3.58(6)$ \\
\hline $5 p^{5} 7 p$ & ${ }^{1} S$ & 0 & $5 p^{5} 6 s$ & ${ }^{1} P^{o}$ & 1 & 897.76 & 723.94 & -2.582 & -2.848 & $2.17(7)$ & $1.81(7)$ \\
\hline $5 p^{5} 7 p$ & ${ }^{3} P$ & 0 & $5 p^{5} 6 s$ & ${ }^{1} P^{o}$ & 1 & 768.34 & 844.52 & -3.600 & -2.230 & $2.84(6)$ & $5.50(7)$ \\
\hline $5 p^{5} 7 p$ & ${ }^{3} S$ & 1 & $5 p^{5} 6 s$ & ${ }^{3} P^{o}$ & 0 & 1146.53 & 1165.60 & -2.510 & -1.955 & $5.22(6)$ & $1.81(7)$ \\
\hline $5 p^{5} 7 p$ & ${ }^{3} S$ & 1 & $5 p^{5} 6 s$ & ${ }^{3} P^{o}$ & 1 & 1162.75 & 1182.90 & -2.501 & -1.793 & $5.19(6)$ & $2.56(7)$ \\
\hline $5 p^{5} 7 p$ & ${ }^{3} P$ & 2 & $5 p^{5} 6 s$ & ${ }^{3} P^{o}$ & 1 & 1121.88 & 1101.70 & -2.856 & -2.224 & $1.48(6)$ & $6.56(6)$ \\
\hline $5 p^{5} 7 p$ & ${ }^{1} S$ & 0 & $5 p^{5} 6 s$ & ${ }^{3} P^{o}$ & 1 & 1098.59 & 848.68 & -2.930 & -3.130 & $6.50(6)$ & $6.86(6)$ \\
\hline $5 p^{5} 7 p$ & ${ }^{3} D$ & 1 & $5 p^{5} 6 s$ & ${ }^{3} P^{o}$ & 1 & 929.08 & 921.16 & -2.949 & -1.989 & $2.90(6)$ & $2.69(7)$ \\
\hline $5 p^{5} 7 p$ & ${ }^{3} P$ & 1 & $5 p^{5} 6 s$ & ${ }^{3} P^{o}$ & 1 & 913.98 & 904.40 & -3.348 & -3.488 & $1.19(6)$ & $8.84(5)$ \\
\hline $5 p^{5} 7 p$ & ${ }^{3} P$ & 0 & $5 p^{5} 6 s$ & ${ }^{3} P^{o}$ & 1 & 910.85 & 1019.30 & -2.742 & -2.946 & $1.46(7)$ & $7.27(6)$ \\
\hline $5 p^{5} 7 p$ & ${ }^{3} S$ & 1 & $5 p^{5} 6 d$ & ${ }^{3} P^{o}$ & 0 & 4480.04 & 3947.40 & -0.391 & -0.500 & $4.50(7)$ & $4.52(7)$ \\
\hline $5 p^{5} 7 p$ & ${ }^{3} D$ & 1 & $5 p^{5} 6 d$ & ${ }^{3} P^{o}$ & 0 & 2275.23 & 2026.20 & -1.420 & -3.241 & $1.63(7)$ & $3.11(5)$ \\
\hline $5 p^{5} 7 p$ & ${ }^{3} P$ & 1 & $5 p^{5} 6 d$ & ${ }^{3} P^{o}$ & 0 & 2186.75 & 1946.90 & -2.394 & -2.426 & $1.88(6)$ & $2.20(6)$ \\
\hline $5 p^{5} 7 p$ & ${ }^{3} S$ & 1 & $5 p^{5} 6 d$ & ${ }^{3} P^{o}$ & 1 & 4632.56 & 4142.70 & -0.069 & -0.128 & $8.85(7)$ & $9.64(7)$ \\
\hline $5 p^{5} 7 p$ & ${ }^{3} D$ & 2 & $5 p^{5} 6 d$ & ${ }^{3} P^{o}$ & 1 & 4470.84 & 3706.10 & -0.716 & -0.895 & $1.28(7)$ & $1.24(7)$ \\
\hline $5 p^{5} 7 p$ & ${ }^{1} P$ & 1 & $5 p^{5} 6 d$ & ${ }^{3} P^{o}$ & 1 & 4150.30 & 3399.80 & -1.781 & -1.980 & $2.14(6)$ & $2.01(6)$ \\
\hline $5 p^{5} 7 p$ & ${ }^{3} P$ & 2 & $5 p^{5} 6 d$ & ${ }^{3} P^{o}$ & 1 & 4045.31 & 3293.30 & -0.750 & -0.892 & $1.45(7)$ & $1.58(7)$ \\
\hline $5 p^{5} 7 p$ & ${ }^{1} S$ & 0 & $5 p^{5} 6 d$ & ${ }^{3} P^{o}$ & 1 & 3758.10 & 1741.30 & -1.977 & -2.307 & $4.98(6)$ & $1.09(7)$ \\
\hline $5 p^{5} 7 p$ & ${ }^{3} D$ & 1 & $5 p^{5} 6 d$ & ${ }^{3} P^{o}$ & 1 & 2313.92 & 2076.50 & -1.351 & -2.335 & $1.85(7)$ & $2.38(6)$ \\
\hline $5 p^{5} 7 p$ & ${ }^{3} P$ & 1 & $5 p^{5} 6 d$ & ${ }^{3} P^{o}$ & 1 & 2222.47 & 1993.20 & -2.228 & -2.509 & $2.66(6)$ & $1.73(6)$ \\
\hline $5 p^{5} 7 p$ & ${ }^{3} P$ & 0 & $5 p^{5} 6 d$ & ${ }^{3} P^{o}$ & 1 & 2204.03 & 2652.00 & -3.013 & -2.123 & $1.33(6)$ & $7.15(6)$ \\
\hline $5 p^{5} 7 p$ & ${ }^{3} D$ & 3 & $5 p^{5} 6 d$ & ${ }^{3} F^{o}$ & 4 & 4422.54 & 3841.40 & 0.572 & 0.471 & $1.82(8)$ & $1.91(8)$ \\
\hline $5 p^{5} 7 p$ & ${ }^{3} D$ & 2 & $5 p^{5} 6 d$ & ${ }^{3} F^{o}$ & 3 & 4719.44 & 4035.40 & 0.384 & 0.289 & $1.45(8)$ & $1.59(8)$ \\
\hline $5 p^{5} 7 p$ & ${ }^{3} D$ & 3 & $5 p^{5} 6 d$ & ${ }^{3} F^{o}$ & 3 & 4484.07 & 3954.10 & -0.501 & -0.500 & $1.50(7)$ & $1.93(7)$ \\
\hline $5 p^{5} 7 p$ & ${ }^{3} S$ & 1 & $5 p^{5} 6 d$ & ${ }^{3} P^{o}$ & 2 & 4904.23 & 4496.40 & -0.116 & -0.087 & $7.08(7)$ & $9.00(7)$ \\
\hline $5 p^{5} 7 p$ & ${ }^{3} D$ & 2 & $5 p^{5} 6 d$ & ${ }^{3} P^{o}$ & 2 & 4723.35 & 3986.60 & -1.379 & -1.515 & $2.50(6)$ & $2.56(6)$ \\
\hline $5 p^{5} 7 p$ & ${ }^{3} D$ & 3 & $5 p^{5} 6 d$ & ${ }^{3} P^{o}$ & 2 & 4487.60 & 3907.30 & -0.441 & -0.584 & $1.72(7)$ & $1.63(7)$ \\
\hline $5 p^{5} 7 p$ & ${ }^{1} P$ & 1 & $5 p^{5} 6 d$ & ${ }^{3} P^{o}$ & 2 & 4367.03 & 3634.40 & -0.619 & -1.150 & $2.80(7)$ & $1.19(7)$ \\
\hline $5 p^{5} 7 p$ & ${ }^{3} P$ & 2 & $5 p^{5} 6 d$ & ${ }^{3} P^{o}$ & 2 & 4250.94 & 3512.90 & -0.136 & -0.311 & $5.39(7)$ & $5.28(7)$ \\
\hline $5 p^{5} 7 p$ & ${ }^{3} D$ & 1 & $5 p^{5} 6 d$ & ${ }^{3} P^{o}$ & 2 & 2379.76 & 2161.70 & -2.261 & -2.803 & $2.15(6)$ & $7.48(5)$ \\
\hline $5 p^{5} 7 p$ & ${ }^{3} P$ & 1 & $5 p^{5} 6 d$ & ${ }^{3} P^{o}$ & 2 & 2283.15 & 2071.60 & -2.226 & -2.402 & $2.54(6)$ & $2.05(6)$ \\
\hline $5 p^{5} 7 p$ & ${ }^{3} S$ & 1 & $5 p^{5} 6 d$ & ${ }^{1} D^{o}$ & 2 & 5214.46 & 4960.70 & -1.145 & -2.846 & $5.86(6)$ & $1.29(5)$ \\
\hline $5 p^{5} 7 p$ & ${ }^{3} D$ & 2 & $5 p^{5} 6 d$ & ${ }^{1} D^{o}$ & 2 & 5010.45 & 4347.50 & -0.247 & -0.271 & $3.01(7)$ & $3.78(7)$ \\
\hline $5 p^{5} 7 p$ & ${ }^{1} P$ & 1 & $5 p^{5} 6 d$ & ${ }^{1} D^{o}$ & 2 & 4611.33 & 3931.90 & 0.107 & 0.000 & $1.34(8)$ & $1.44(8)$ \\
\hline $5 p^{5} 7 p$ & ${ }^{3} D$ & 1 & $5 p^{5} 6 d$ & ${ }^{1} D^{o}$ & 2 & 2450.51 & 2263.50 & -2.393 & -2.436 & $1.50(6)$ & $1.59(6)$ \\
\hline $5 p^{5} 7 p$ & ${ }^{3} D$ & 3 & $5 p^{5} 6 d$ & ${ }^{3} D^{o}$ & 3 & 4856.10 & 4462.50 & -0.311 & -0.436 & $1.97(7)$ & $1.76(7)$ \\
\hline $5 p^{5} 7 p$ & ${ }^{3} P$ & 2 & $5 p^{5} 6 d$ & ${ }^{3} D^{o}$ & 3 & 4580.18 & 3955.30 & 0.359 & 0.216 & $1.45(8)$ & $1.40(8)$ \\
\hline
\end{tabular}


TABLE IV (cont.)

\begin{tabular}{|c|c|c|c|c|c|c|c|c|c|c|c|}
\hline \multicolumn{3}{|c|}{ Upper level } & \multicolumn{3}{|c|}{ Lower level } & \multicolumn{2}{|c|}{$\lambda[\bar{\lambda}[\AA]$} & \multicolumn{2}{|c|}{$\log (g f)$} & \multicolumn{2}{|c|}{$A_{j i}\left[\mathrm{~s}^{-1}\right]$} \\
\hline Conf. & Term & $J$ & Conf. & Term & $J$ & HFR & MCDF & HFR & MCDF & HFR & MCDF \\
\hline $5 p^{5} 7 p$ & ${ }^{3} D$ & 2 & $5 p^{5} 6 d$ & ${ }^{3} D^{o}$ & 3 & 2376.25 & 4566.20 & -2.056 & -1.293 & $2.08(6)$ & $3.26(6)$ \\
\hline $5 p^{5} 7 p$ & ${ }^{1} S$ & 0 & $5 p^{5} 6 d$ & ${ }^{1} P^{o}$ & 1 & 4803.85 & 4654.30 & -0.097 & -1.073 & $2.31(8)$ & $7.79(7)$ \\
\hline $5 p^{5} 7 p$ & ${ }^{3} D$ & 1 & $5 p^{5} 6 d$ & ${ }^{3} F^{o}$ & 2 & 4778.94 & 4044.30 & 0.202 & 0.149 & $1.55(8)$ & $1.92(8)$ \\
\hline $5 p^{5} 7 p$ & ${ }^{3} P$ & 1 & $5 p^{5} 6 d$ & ${ }^{3} F^{o}$ & 2 & 4404.62 & 3740.00 & -1.480 & -1.657 & $3.80(6)$ & $3.50(6)$ \\
\hline $5 p^{5} 7 p$ & ${ }^{3} D$ & 2 & $5 p^{5} 6 d$ & ${ }^{3} P^{o}$ & 2 & 4499.25 & 4762.40 & -0.697 & 0.086 & $1.32(7)$ & $7.17(7)$ \\
\hline $5 p^{5} 7 p$ & ${ }^{3} D$ & 1 & $5 p^{5} 6 d$ & ${ }^{3} D^{o}$ & 1 & 5467.82 & 2892.50 & -0.556 & -1.190 & $2.07(7)$ & $1.72(7)$ \\
\hline $5 p^{5} 7 p$ & ${ }^{3} D$ & 2 & $5 p^{5} 6 d$ & ${ }^{3} D^{o}$ & 1 & 4989.55 & 7465.20 & -1.256 & -1.578 & $2.97(6)$ & $6.33(5)$ \\
\hline $5 p^{5} 7 p$ & ${ }^{3} P$ & 1 & $5 p^{5} 6 d$ & ${ }^{3} D^{o}$ & 1 & 4983.28 & 2733.50 & -0.660 & -1.276 & $1.96(7)$ & $1.57(7)$ \\
\hline $5 p^{5} 7 p$ & ${ }^{3} P$ & 0 & $5 p^{5} 6 d$ & ${ }^{3} D^{o}$ & 1 & 4891.54 & 4145.80 & -0.164 & -0.392 & $1.91(8)$ & $1.57(8)$ \\
\hline $5 p^{5} 7 p$ & ${ }^{3} S$ & 1 & $5 p^{5} 7 s$ & ${ }^{3} P^{o}$ & 2 & 6915.15 & 5508.40 & 0.184 & 0.157 & $7.10(7)$ & $1.05(8)$ \\
\hline $5 p^{5} 7 p$ & ${ }^{3} D$ & 3 & $5 p^{5} 7 s$ & ${ }^{3} P^{o}$ & 2 & 6114.69 & 4649.70 & 0.616 & 0.671 & $1.05(8)$ & $2.06(8)$ \\
\hline $5 p^{5} 7 p$ & ${ }^{1} P$ & 1 & $5 p^{5} 7 s$ & ${ }^{3} P^{o}$ & 2 & 5892.99 & 4268.30 & -1.688 & -0.692 & $1.31(6)$ & $2.48(7)$ \\
\hline $5 p^{5} 7 p$ & ${ }^{3} P$ & 2 & $5 p^{5} 7 s$ & ${ }^{3} P^{o}$ & 2 & 5683.55 & 4101.70 & 0.321 & 0.419 & $8.65(7)$ & $2.08(8)$ \\
\hline $5 p^{5} 7 p$ & ${ }^{3} P$ & 1 & $5 p^{5} 7 s$ & ${ }^{3} P^{o}$ & 2 & 2640.64 & 2263.20 & -2.122 & -1.254 & $2.41(6)$ & $2.42(7)$ \\
\hline $5 p^{5} 7 p$ & ${ }^{3} D$ & 2 & $5 p^{5} 7 s$ & ${ }^{1} P^{o}$ & 1 & 6882.98 & 4993.20 & 0.203 & 0.295 & $4.50(7)$ & $1.05(8)$ \\
\hline $5 p^{5} 7 p$ & ${ }^{1} P$ & 1 & $5 p^{5} 7 s$ & ${ }^{1} P^{o}$ & 1 & 6151.55 & 4452.70 & 0.282 & 0.311 & $1.13(8)$ & $2.30(8)$ \\
\hline $5 p^{5} 7 p$ & ${ }^{3} P$ & 2 & $5 p^{5} 7 s$ & ${ }^{1} P^{o}$ & 1 & 5923.69 & 4271.80 & 0.098 & 0.140 & $4.77(7)$ & $1.01(8)$ \\
\hline $5 p^{5} 7 p$ & ${ }^{1} S$ & 0 & $5 p^{5} 7 s$ & ${ }^{1} P^{o}$ & 1 & 5327.49 & 1981.20 & -0.221 & -1.025 & $1.41(8)$ & $1.60(8)$ \\
\hline $5 p^{5} 7 p$ & ${ }^{3} D$ & 1 & $5 p^{5} 7 s$ & ${ }^{3} P^{o}$ & 0 & 6832.31 & 4869.00 & -0.216 & -0.083 & $2.90(7)$ & $7.74(7)$ \\
\hline $5 p^{5} 7 p$ & ${ }^{3} P$ & 1 & $5 p^{5} 7 s$ & ${ }^{3} P^{o}$ & 0 & 6092.13 & 4434.70 & 0.038 & 0.091 & $6.54(7)$ & $1.39(8)$ \\
\hline $5 p^{5} 7 p$ & ${ }^{3} D$ & 1 & $5 p^{5} 7 s$ & ${ }^{3} P^{o}$ & 1 & 7011.54 & 4987.50 & -0.013 & 0.031 & $4.39(7)$ & $9.60(7)$ \\
\hline $5 p^{5} 7 p$ & ${ }^{3} P$ & 1 & $5 p^{5} 7 s$ & ${ }^{3} P^{o}$ & 1 & 6234.23 & 4532.80 & -0.151 & -0.076 & $4.04(7)$ & $9.09(7)$ \\
\hline $5 p^{5} 8 s$ & ${ }^{3} P^{o}$ & 2 & $5 p^{5} 7 p$ & ${ }^{3} S$ & 1 & 3776.87 & 4883.50 & -0.072 & -1.031 & $7.93(7)$ & $5.21(6)$ \\
\hline $5 p^{5} 8 s$ & ${ }^{3} P^{o}$ & 2 & $5 p^{5} 7 p$ & ${ }^{3} D$ & 2 & 3891.64 & 5671.10 & -0.140 & -1.378 & $6.37(7)$ & $1.74(6)$ \\
\hline $5 p^{5} 8 s$ & ${ }^{3} P^{o}$ & 2 & $5 p^{5} 7 p$ & ${ }^{3} D$ & 3 & 4067.70 & 5839.70 & 0.326 & -0.565 & $1.71(8)$ & $1.07(7)$ \\
\hline $5 p^{5} 8 s$ & ${ }^{3} P^{o}$ & 2 & $5 p^{5} 7 p$ & ${ }^{3} P$ & 2 & 4283.88 & 7017.00 & 0.084 & -1.018 & $8.82(7)$ & $2.60(6)$ \\
\hline $5 p^{5} 8 s$ & ${ }^{1} P^{o}$ & 1 & $5 p^{5} 7 p$ & ${ }^{3} D$ & 2 & 3848.45 & 5537.00 & 0.028 & -1.127 & $1.60(8)$ & $5.41(6)$ \\
\hline $5 p^{5} 8 s$ & ${ }^{1} P^{o}$ & 1 & $5 p^{5} 7 p$ & ${ }^{1} P$ & 1 & 4122.52 & 6398.20 & -0.281 & -1.102 & $6.85(7)$ & $4.30(6)$ \\
\hline $5 p^{5} 8 s$ & ${ }^{1} P^{o}$ & 1 & $5 p^{5} 7 p$ & ${ }^{3} P$ & 2 & 4231.61 & 6813.00 & -0.162 & -1.248 & $8.55(7)$ & $2.71(6)$ \\
\hline $5 p^{5} 8 s$ & ${ }^{1} P^{o}$ & 1 & $5 p^{5} 7 p$ & ${ }^{1} S$ & 0 & 4599.29 & 8072.80 & -0.092 & -2.788 & $8.50(7)$ & $1.67(5)$ \\
\hline $5 p^{5} 8 s$ & ${ }^{3} P^{o}$ & 0 & $5 p^{5} 7 p$ & ${ }^{3} D$ & 1 & 3840.50 & 5488.20 & -0.430 & -1.453 & $1.68(8)$ & $7.81(6)$ \\
\hline $5 p^{5} 8 s$ & ${ }^{3} P^{o}$ & 0 & $5 p^{5} 7 p$ & ${ }^{3} P$ & 1 & 4122.01 & 6169.20 & -0.239 & -1.180 & $2.26(8)$ & $1.16(7)$ \\
\hline $5 p^{5} 8 s$ & ${ }^{3} P^{o}$ & 1 & $5 p^{5} 7 p$ & ${ }^{3} D$ & 2 & 2128.77 & 2525.40 & -1.593 & -2.843 & $1.25(7)$ & $5.00(5)$ \\
\hline $5 p^{5} 8 s$ & ${ }^{3} P^{o}$ & 1 & $5 p^{5} 7 p$ & ${ }^{1} P$ & 1 & 2210.04 & 2690.50 & -1.684 & -2.990 & $9.42(6)$ & $3.14(5)$ \\
\hline $5 p^{5} 8 s$ & ${ }^{3} P^{o}$ & 1 & $5 p^{5} 7 p$ & ${ }^{1} S$ & 0 & 2340.08 & 10928.00 & -1.936 & -1.924 & $4.70(6)$ & $2.22(5)$ \\
\hline $5 p^{5} 8 s$ & ${ }^{3} P^{o}$ & 1 & $5 p^{5} 7 p$ & ${ }^{3} D$ & 1 & 3827.61 & 5428.30 & -0.280 & -1.393 & $7.97(7)$ & $3.05(6)$ \\
\hline $5 p^{5} 8 s$ & ${ }^{3} P^{o}$ & 1 & $5 p^{5} 7 p$ & ${ }^{3} P$ & 1 & 4107.17 & 6093.60 & -0.478 & -1.384 & $4.38(7)$ & $2.48(6)$ \\
\hline
\end{tabular}


TABLE V

The wavelengths $\lambda(\AA)$, logarithmic weighted oscillator strengths $\log (g f)$, and weighted transition probabilities $g A_{j i}$ $\left(\mathrm{s}^{-1}\right)$, for some electric quadruple (E2) and magnetic dipole (M1) transitions in La IV. Numbers in brackets represent powers of 10 .

\begin{tabular}{|c|c|c|c|c|c|c|c|c|c|c|c|c|}
\hline \multicolumn{6}{|c|}{$\overline{\text { Transitions }}$} & \multirow{3}{*}{ Type } & \multicolumn{2}{|c|}{$\lambda[\AA]$} & \multicolumn{2}{|c|}{$\log (g f)$} & \multicolumn{2}{|c|}{$g A_{j i}\left[\mathrm{~s}^{-1}\right]$} \\
\hline \multicolumn{3}{|c|}{ Lower levels } & \multicolumn{3}{|c|}{ Upper levels } & & HFR & $M C D F$ & HFR & MCDF & HFR & $M C D F$ \\
\hline Conf. & Term & $J$ & Conf. & Term & $J$ & & HFR & MCDF & HFK & MCDF & & \\
\hline $5 p^{5} 5 d$ & ${ }^{3} P^{o}$ & 2 & $5 p^{5} 6 d$ & ${ }^{3} P^{o}$ & 0 & $\mathrm{E} 2$ & 823.94 & 871.97 & -5.887 & -5.748 & $1.28(4)$ & $7.83(4)$ \\
\hline $5 p^{5} 6 s$ & ${ }^{3} P^{o}$ & 2 & $5 p^{5} 6 d$ & ${ }^{3} P^{o}$ & 0 & $\mathrm{E} 2$ & 1164.25 & 1228.00 & -5.417 & -5.411 & $1.88(4)$ & $8.59(4)$ \\
\hline $5 p^{5} 5 d$ & ${ }^{3} P^{o}$ & 1 & $5 p^{5} 6 d$ & ${ }^{3} P^{o}$ & 1 & $\mathrm{E} 2$ & 790.04 & 828.25 & -5.927 & -5.760 & $1.26(4)$ & $1.69(4)$ \\
\hline $5 p^{5} 5 d$ & ${ }^{3} P^{o}$ & 2 & $5 p^{5} 6 d$ & ${ }^{3} P^{o}$ & 1 & $\mathrm{E} 2$ & 818.98 & 862.98 & -5.852 & -5.663 & $1.40(4)$ & $3.24(4)$ \\
\hline $5 p^{5} 6 s$ & ${ }^{3} P^{o}$ & 2 & $5 p^{5} 6 d$ & ${ }^{3} P^{o}$ & 1 & $\mathrm{E} 2$ & 1154.37 & 1210.20 & -4.957 & -4.964 & $5.53(4)$ & $8.24(4)$ \\
\hline $5 p^{5} 5 d$ & ${ }^{3} P^{o}$ & 2 & $5 p^{5} 6 d$ & ${ }^{3} F^{o}$ & 4 & $\mathrm{E} 2$ & 813.20 & 852.25 & -5.724 & -5.554 & $1.91(4)$ & $1.43(4)$ \\
\hline $5 p^{5} 5 d$ & ${ }^{3} F^{o}$ & 4 & $5 p^{5} 6 d$ & ${ }^{3} F^{o}$ & 4 & $\mathrm{E} 2$ & 819.02 & 864.18 & -5.187 & -4.996 & $6.46(4)$ & $9.02(4)$ \\
\hline $5 p^{5} 5 d$ & ${ }^{3} F^{o}$ & 3 & $5 p^{5} 6 d$ & ${ }^{3} F^{o}$ & 4 & $\mathrm{E} 2$ & 827.43 & 882.38 & -5.627 & -5.449 & $2.30(4)$ & $2.37(4)$ \\
\hline $5 p^{5} 5 d$ & ${ }^{3} D^{o}$ & 3 & $5 p^{5} 6 d$ & ${ }^{3} F^{o}$ & 4 & $\mathrm{E} 2$ & 889.61 & 966.67 & -5.795 & -5.543 & $1.35(4)$ & $1.59(4)$ \\
\hline $5 p^{5} 6 s$ & ${ }^{3} P^{o}$ & 2 & $5 p^{5} 6 d$ & ${ }^{3} F^{o}$ & 4 & $\mathrm{E} 2$ & 1142.92 & 1189.20 & -4.467 & -4.480 & $1.74(5)$ & $8.68(4)$ \\
\hline $5 p^{5} 5 d$ & ${ }^{3} F^{o}$ & 4 & $5 p^{5} 6 d$ & ${ }^{3} F^{o}$ & 3 & $\mathrm{E} 2$ & 816.94 & 858.68 & -5.867 & -5.550 & $1.36(4)$ & $3.28(4)$ \\
\hline $5 p^{5} 5 d$ & ${ }^{3} F^{o}$ & 3 & $5 p^{5} 6 d$ & ${ }^{3} F^{o}$ & 3 & $\mathrm{E} 2$ & 825.31 & 876.64 & -5.586 & -5.441 & $2.54(4)$ & $3.15(4)$ \\
\hline $5 p^{5} 5 d$ & ${ }^{3} D^{o}$ & 3 & $5 p^{5} 6 d$ & ${ }^{3} F^{o}$ & 3 & $\mathrm{E} 2$ & 887.16 & 959.79 & -5.805 & -5.653 & $1.33(4)$ & $1.61(4)$ \\
\hline $5 p^{5} 6 s$ & ${ }^{3} P^{o}$ & 2 & $5 p^{5} 6 d$ & ${ }^{3} F^{o}$ & 3 & E2 & 1138.88 & 1178.80 & -5.038 & -4.963 & $4.71(4)$ & $3.73(4)$ \\
\hline $5 p^{5} 6 s$ & ${ }^{1} P^{o}$ & 1 & $5 p^{5} 6 d$ & ${ }^{3} F^{o}$ & 3 & $\mathrm{E} 2$ & 1163.36 & 1206.20 & -4.770 & -4.818 & $8.37(4)$ & $2.99(4)$ \\
\hline $5 p^{5} 5 d$ & ${ }^{3} P^{o}$ & 0 & $5 p^{5} 6 d$ & ${ }^{3} P^{o}$ & 2 & E2 & 768.90 & 799.28 & -5.996 & -5.793 & $1.14(4)$ & $3.37(3)$ \\
\hline $5 p^{5} 5 d$ & ${ }^{3} P^{o}$ & 1 & $5 p^{5} 6 d$ & ${ }^{3} P^{o}$ & 2 & $\mathrm{E} 2$ & 782.65 & 815.42 & -5.957 & -5.713 & $1.20(4)$ & $1.17(4)$ \\
\hline $5 p^{5} 5 d$ & ${ }^{3} D^{o}$ & 3 & $5 p^{5} 6 d$ & ${ }^{3} P^{o}$ & 2 & $\mathrm{E} 2$ & 887.02 & 962.59 & -5.628 & -5.461 & $2.00(4)$ & $3.49(4)$ \\
\hline $5 p^{5} 6 s$ & ${ }^{3} P^{o}$ & 2 & $5 p^{5} 6 d$ & ${ }^{3} P^{o}$ & 2 & E2 & 1138.65 & 1183.00 & -4.833 & -4.862 & $7.55(4)$ & $6.55(4)$ \\
\hline $5 p^{5} 6 s$ & ${ }^{1} P^{o}$ & 1 & $5 p^{5} 6 d$ & ${ }^{3} P^{o}$ & 2 & $\mathrm{E} 2$ & 1163.12 & 1210.60 & -5.392 & -5.391 & $2.00(4)$ & $1.11(4)$ \\
\hline $5 p^{5} 5 d$ & ${ }^{3} F^{o}$ & 3 & $5 p^{5} 6 d$ & ${ }^{1} D^{o}$ & 2 & $\mathrm{E} 2$ & 817.01 & 863.18 & -5.654 & -5.436 & $2.22(4)$ & $4.60(4)$ \\
\hline $5 p^{5} 5 d$ & ${ }^{3} D^{o}$ & 1 & $5 p^{5} 6 d$ & ${ }^{1} D^{o}$ & 2 & $\mathrm{E} 2$ & 938.86 & 1027.00 & -5.799 & -5.582 & $1.20(4)$ & $9.94(3)$ \\
\hline $5 p^{5} 6 s$ & ${ }^{3} P^{o}$ & 2 & $5 p^{5} 6 d$ & ${ }^{1} D^{o}$ & 2 & E2 & 1123.14 & 1154.60 & -5.401 & -5.434 & $2.10(4)$ & $1.84(4)$ \\
\hline $5 p^{5} 6 s$ & ${ }^{1} P^{o}$ & 1 & $5 p^{5} 6 d$ & ${ }^{1} D^{o}$ & 2 & $\mathrm{E} 2$ & 1146.94 & 1180.90 & -4.829 & -4.820 & $7.52(4)$ & $4.34(4)$ \\
\hline $5 p^{5} 5 d$ & ${ }^{3} P^{o}$ & 2 & $5 p^{5} 6 d$ & ${ }^{3} D^{o}$ & 3 & $\mathrm{E} 2$ & 800.07 & 826.72 & -5.873 & -5.668 & $1.40(4)$ & $1.50(4)$ \\
\hline $5 p^{5} 5 d$ & ${ }^{3} F^{o}$ & 4 & $5 p^{5} 6 d$ & ${ }^{3} D^{o}$ & 3 & $\mathrm{E} 2$ & 805.70 & 837.95 & -5.648 & -5.475 & $2.31(4)$ & $4.09(4)$ \\
\hline $5 p^{5} 5 d$ & ${ }^{3} D^{o}$ & 3 & $5 p^{5} 6 d$ & ${ }^{3} D^{o}$ & 3 & $\mathrm{E} 2$ & 873.91 & 933.97 & -5.701 & -5.392 & $1.74(4)$ & $3.10(4)$ \\
\hline $5 p^{5} 6 s$ & ${ }^{3} P^{o}$ & 2 & $5 p^{5} 6 d$ & ${ }^{3} D^{o}$ & 3 & $\mathrm{E} 2$ & 1117.14 & 1140.10 & -4.764 & -4.863 & $9.21(4)$ & $5.02(4)$ \\
\hline $5 p^{5} 6 s$ & ${ }^{1} P^{o}$ & 1 & $5 p^{5} 6 d$ & ${ }^{3} D^{o}$ & 3 & $\mathrm{E} 2$ & 1140.69 & 1165.70 & -5.032 & -4.901 & $4.76(4)$ & $2.64(4)$ \\
\hline $5 p^{5} 5 d$ & ${ }^{3} F^{o}$ & 2 & $5 p^{5} 6 d$ & ${ }^{1} P^{o}$ & 1 & $\mathrm{E} 2$ & 816.07 & 695.35 & -5.912 & -7.165 & $1.23(4)$ & $1.57(3)$ \\
\hline $5 p^{5} 6 s$ & ${ }^{1} P^{o}$ & 1 & $5 p^{5} 6 d$ & ${ }^{1} P^{o}$ & 1 & E2 & 1104.09 & 857.29 & -4.979 & -5.668 & $5.74(4)$ & $1.95(4)$ \\
\hline $5 p^{5} 5 d$ & ${ }^{3} F^{o}$ & 2 & $5 p^{5} 6 d$ & ${ }^{3} F^{o}$ & 2 & $\mathrm{E} 2$ & 818.18 & 761.61 & -5.467 & -6.458 & $3.40(4)$ & $4.01(3)$ \\
\hline $5 p^{5} 5 d$ & ${ }^{1} F^{o}$ & 3 & $5 p^{5} 6 d$ & ${ }^{3} F^{o}$ & 2 & E2 & 858.93 & 920.89 & -5.863 & -5.638 & $1.24(4)$ & $2.54(4)$ \\
\hline $5 p^{5} 6 s$ & ${ }^{3} P^{o}$ & 0 & $5 p^{5} 6 d$ & ${ }^{3} F^{o}$ & 2 & E2 & 1137.33 & 1175.30 & -5.058 & -5.034 & $4.51(4)$ & $8.93(3)$ \\
\hline $5 p^{5} 6 s$ & ${ }^{3} P^{o}$ & 1 & $5 p^{5} 6 d$ & ${ }^{3} F^{o}$ & 2 & E2 & 1153.29 & 1192.80 & -5.012 & -5.017 & $4.87(4)$ & $2.70(4)$ \\
\hline $5 p^{5} 5 d$ & ${ }^{3} D^{o}$ & 2 & $5 p^{5} 6 d$ & ${ }^{3} P^{o}$ & 2 & $\mathrm{E} 2$ & 838.19 & 1118.00 & -5.501 & -5.751 & $2.99(4)$ & $9.48(3)$ \\
\hline $5 p^{5} 6 s$ & ${ }^{3} P^{o}$ & 1 & $5 p^{5} 6 d$ & ${ }^{3} D^{o}$ & 1 & $\mathrm{E} 2$ & 1119.26 & 1351.60 & -4.950 & -5.859 & $5.97(4)$ & $5.05(3)$ \\
\hline $5 p^{6}$ & ${ }^{1} S$ & 0 & $5 p^{5} 6 p$ & ${ }^{3} D$ & 2 & $\mathrm{E} 2$ & 385.19 & 435.50 & -4.827 & -5.041 & $6.70(5)$ & $6.40(4)$ \\
\hline $5 p^{6}$ & ${ }^{1} S$ & 0 & $5 p^{5} 7 p$ & ${ }^{3} D$ & 2 & $\mathrm{E} 2$ & 303.11 & 326.48 & -5.115 & -4.904 & $5.57(5)$ & $1.56(5)$ \\
\hline $5 p^{6}$ & ${ }^{1} S$ & 0 & $5 p^{5} 6 p$ & ${ }^{3} P$ & 2 & $\mathrm{E} 2$ & 417.15 & 424.38 & -5.014 & -5.264 & $3.71(5)$ & $4.03(4)$ \\
\hline $5 p^{6}$ & ${ }^{1} S$ & 0 & $5 p^{5} 7 p$ & ${ }^{3} P$ & 2 & $\mathrm{E} 2$ & 322.93 & 322.91 & -5.564 & -5.079 & $1.75(5)$ & $1.07(5)$ \\
\hline $5 p^{5} 6 p$ & ${ }^{3} S$ & 1 & $5 p^{5} 7 p$ & ${ }^{3} D$ & 3 & $\mathrm{E} 2$ & 1292.40 & 1241.10 & -5.182 & -5.055 & $2.63(4)$ & $1.63(4)$ \\
\hline $5 p^{5} 6 p$ & ${ }^{3} D$ & 3 & $5 p^{5} 7 p$ & ${ }^{3} S$ & 1 & $\mathrm{E} 2$ & 1432.32 & 1406.30 & -5.282 & -5.050 & $1.70(4)$ & $7.00(4)$ \\
\hline $5 p^{5} 6 p$ & ${ }^{3} D$ & 2 & $5 p^{5} 7 p$ & ${ }^{3} P$ & 2 & $\mathrm{E} 2$ & 1309.15 & 1249.00 & -5.382 & -5.200 & $1.62(4)$ & $2.69(4)$ \\
\hline $5 p^{5} 6 p$ & ${ }^{3} D$ & 3 & $5 p^{5} 7 p$ & ${ }^{3} D$ & 3 & $\mathrm{E} 2$ & 1394.51 & 1343.00 & -5.384 & -5.176 & $1.42(4)$ & $2.46(4)$ \\
\hline $5 p^{5} 6 p$ & ${ }^{3} D$ & 2 & $5 p^{5} 7 p$ & ${ }^{1} P$ & 1 & $\mathrm{E} 2$ & 1319.96 & 1264.00 & -5.518 & -5.394 & $1.16(4)$ & $2.80(4)$ \\
\hline $5 p^{5} 6 p$ & ${ }^{3} D$ & 3 & $5 p^{5} 7 p$ & ${ }^{3} P$ & 2 & $\mathrm{E} 2$ & 1370.80 & 1293.10 & -5.491 & -5.150 & $1.15(4)$ & $3.95(4)$ \\
\hline $5 p^{5} 6 p$ & ${ }^{3} D$ & 2 & $5 p^{5} 7 p$ & ${ }^{3} D$ & 3 & $\mathrm{E} 2$ & 1330.76 & 1295.50 & -5.534 & -5.379 & $1.10(4)$ & $1.18(4)$ \\
\hline $5 p^{5} 6 p$ & ${ }^{3} D$ & 2 & $5 p^{5} 7 p$ & ${ }^{3} D$ & 2 & $\mathrm{E} 2$ & 1422.35 & 1304.10 & -5.49 & -6.368 & $1.07(4)$ & $1.68(3)$ \\
\hline $5 p^{5} 5 d$ & ${ }^{3} P^{o}$ & 1 & $5 p^{5} 5 d$ & ${ }^{3} D^{o}$ & 1 & M1 & 4450.02 & 3656.90 & -6.512 & -6.567 & $1.04(2)$ & $1.35(2)$ \\
\hline
\end{tabular}


TABLE V (cont.)

\begin{tabular}{|c|c|c|c|c|c|c|c|c|c|c|c|c|}
\hline \multicolumn{6}{|c|}{ Transitions } & \multirow{3}{*}{ Type } & \multicolumn{2}{|c|}{$\lambda[\AA]$} & \multicolumn{2}{|c|}{$\log (g f)$} & \multicolumn{2}{|c|}{$g A_{j i}\left[\mathrm{~s}^{-1}\right]$} \\
\hline \multicolumn{3}{|c|}{ Lower levels } & \multicolumn{3}{|c|}{ Upper levels } & & HFR & MCDF & HFR & MCDF & HFR & MCDF \\
\hline Conf. & Term & $J$ & Conf. & Term & $J$ & & $110 \mathrm{n}$ & NUD & $110 \mathrm{n}$ & WIUD & $110 \mathrm{n}$ & NUD \\
\hline $5 p^{5} 5 d$ & ${ }^{3} P^{o}$ & 2 & $5 p^{5} 5 d$ & ${ }^{3} D^{o}$ & 2 & M1 & 3915.14 & 3529.70 & -6.176 & -6.197 & $2.90(2)$ & $3.40(2)$ \\
\hline $5 p^{5} 5 d$ & ${ }^{3} D^{o}$ & 3 & $5 p^{5} 5 d$ & ${ }^{3} D^{o}$ & 2 & M1 & 6675.44 & 6924.40 & -6.038 & -6.073 & $1.37(2)$ & $1.65(2)$ \\
\hline $5 p^{5} 5 d$ & ${ }^{3} F^{o}$ & 4 & $5 p^{5} 5 d$ & ${ }^{1} F^{o}$ & 3 & M1 & 3690.46 & 3436.60 & -5.719 & -5.757 & $9.36(2)$ & $1.27(3)$ \\
\hline $5 p^{5} 5 d$ & ${ }^{3} D^{o}$ & 3 & $5 p^{5} 5 d$ & ${ }^{1} F^{o}$ & 3 & M1 & 5744.13 & 5941.80 & -5.939 & -5.928 & $2.33(2)$ & $2.23(2)$ \\
\hline $5 p^{5} 5 d$ & ${ }^{3} P^{o}$ & 1 & $5 p^{5} 5 d$ & ${ }^{1} P^{o}$ & 1 & M1 & 1737.13 & 1420.10 & -7.123 & -7.297 & $1.66(2)$ & $1.67(2)$ \\
\hline $5 p^{5} 5 d$ & ${ }^{3} F^{o}$ & 2 & $5 p^{5} 5 d$ & ${ }^{1} P^{o}$ & 1 & M1 & 2094.78 & 1737.60 & -7.000 & -7.188 & $1.52(2)$ & $2.39(2)$ \\
\hline $5 p^{5} 5 d$ & ${ }^{3} D^{o}$ & 2 & $5 p^{5} 5 d$ & ${ }^{1} P^{o}$ & 1 & M1 & 3629.47 & 2686.20 & -6.698 & -6.772 & $1.02(2)$ & $2.60(2)$ \\
\hline $5 p^{5} 6 s$ & ${ }^{1} P^{o}$ & 1 & $5 p^{5} 6 s$ & ${ }^{3} P^{o}$ & 0 & M1 & 5223.02 & 5249.00 & -6.202 & -6.201 & $1.54(2)$ & $4.57(2)$ \\
\hline $5 p^{5} 6 s$ & ${ }^{3} P^{o}$ & 2 & $5 p^{5} 6 s$ & ${ }^{3} P^{o}$ & 1 & M1 & 4502.30 & 4498.60 & -5.878 & -5.905 & $4.36(2)$ & $6.84(2)$ \\
\hline $5 p^{5} 6 d$ & ${ }^{3} F^{o}$ & 3 & $5 p^{5} 6 d$ & ${ }^{3} F^{o}$ & 2 & M1 & 4736.33 & 4710.10 & -5.699 & -5.701 & $5.94(2)$ & $8.39(2)$ \\
\hline $5 p^{5} 6 d$ & ${ }^{1} D^{o}$ & 2 & $5 p^{5} 6 d$ & ${ }^{3} F^{o}$ & 2 & M1 & 5029.50 & 5140.80 & -6.203 & -6.176 & $1.65(2)$ & $1.68(2)$ \\
\hline $5 p^{5} 7 s$ & ${ }^{1} P^{o}$ & 1 & $5 p^{5} 7 s$ & ${ }^{3} P^{o}$ & 0 & M1 & 4821.18 & 4838.90 & -6.223 & -6.219 & $1.72(2)$ & $5.16(2)$ \\
\hline $5 p^{5} 7 s$ & ${ }^{3} P^{o}$ & 2 & $5 p^{5} 7 s$ & ${ }^{3} P^{o}$ & 1 & M1 & 4581.02 & 4519.90 & -5.847 & -5.855 & $4.52(2)$ & $7.60(2)$ \\
\hline $5 p^{5} 7 p$ & ${ }^{3} D$ & 2 & $5 p^{5} 7 p$ & ${ }^{3} D$ & 1 & M1 & 4796.26 & 4722.20 & -5.863 & -5.842 & $3.98(2)$ & $7.16(2)$ \\
\hline $5 p^{5} 4 f$ & ${ }^{3} D$ & 3 & $5 p^{5} 4 f$ & ${ }^{3} F$ & 3 & M1 & 4734.01 & 3954.60 & -6.056 & -6.053 & $2.62(2)$ & $3.77(2)$ \\
\hline $5 p^{5} 6 p$ & ${ }^{3} P$ & 2 & $5 p^{5} 6 p$ & ${ }^{3} P$ & 1 & M1 & 5066.04 & 5128.30 & -6.122 & -6.126 & $1.96(2)$ & $3.16(2)$ \\
\hline $5 p^{5} 6 p$ & ${ }^{1} P$ & 1 & $5 p^{5} 6 p$ & ${ }^{3} P$ & 1 & M1 & 4605.92 & 4702.90 & -6.218 & -6.283 & $1.90(2)$ & $1.58(2)$ \\
\hline $5 p^{5} 6 p$ & ${ }^{3} S$ & 1 & $5 p^{5} 6 p$ & ${ }^{1} S$ & 0 & M1 & 3407.02 & 3116.90 & -6.521 & -6.640 & $1.73(2)$ & $4.70(2)$ \\
\hline $5 p^{5} 4 f$ & ${ }^{3} D$ & 2 & $5 p^{5} 4 f$ & ${ }^{1} D$ & 2 & M1 & 3440.81 & 2857.10 & -6.539 & -6.590 & $1.63(2)$ & $2.10(2)$ \\
\hline
\end{tabular}


New electric quadrupole (E2) transitions for La IV.

TABLE VI

\begin{tabular}{|c|c|c|c|c|c|c|c|c|}
\hline \multicolumn{3}{|c|}{ Lower Level } & \multicolumn{3}{|c|}{ Upper Level } & \multirow{2}{*}{$\begin{array}{l}\lambda[\AA] \\
\mathrm{HFR}\end{array}$} & \multirow{2}{*}{$\frac{\log (g f)}{\text { HFR }}$} & \multirow{2}{*}{$\frac{g A_{j i}\left[\mathrm{~s}^{-1}\right.}{\mathrm{HFR}}$} \\
\hline Conf. & Term & $J$ & Conf. & Term & $J$ & & & \\
\hline $5 p^{6}$ & ${ }^{1} S$ & 0 & $5 p^{5} 9 f$ & ${ }^{1} D$ & 2 & 265.93 & -4.401 & $3.75 \times 10^{6}$ \\
\hline $5 p^{6}$ & ${ }^{1} S$ & 0 & $5 p^{5} 5 f$ & ${ }^{1} D$ & 2 & 337.96 & -4.369 & $2.50 \times 10^{6}$ \\
\hline $5 p^{6}$ & ${ }^{1} S$ & 0 & $5 p^{5} 9 f$ & ${ }^{3} F$ & 2 & 251.74 & -4.655 & $2.33 \times 10^{6}$ \\
\hline $5 p^{6}$ & ${ }^{1} S$ & 0 & $5 p^{5} 7 f$ & ${ }^{1} D$ & 2 & 281.45 & -4.572 & $2.26 \times 10^{6}$ \\
\hline $5 p^{6}$ & ${ }^{1} S$ & 0 & $5 p^{5} 5 f$ & ${ }^{3} F$ & 2 & 315.79 & -4.521 & $2.02 \times 10^{6}$ \\
\hline $5 p^{6}$ & ${ }^{1} S$ & 0 & $5 p^{5} 6 f$ & ${ }^{1} D$ & 2 & 298.77 & -4.681 & $1.56 \times 10^{6}$ \\
\hline $5 p^{6}$ & ${ }^{1} S$ & 0 & $5 p^{5} 5 f$ & ${ }^{3} D$ & 2 & 340.09 & -4.845 & $8.24 \times 10^{5}$ \\
\hline $5 p^{6}$ & ${ }^{1} S$ & 0 & $5 p^{5} 8 f$ & ${ }^{1} D$ & 2 & 271.8 & -5.173 & $6.06 \times 10^{5}$ \\
\hline $5 p^{6}$ & ${ }^{1} S$ & 0 & $5 p^{5} 7 f$ & ${ }^{3} F$ & 2 & 265.57 & -5.23 & $5.58 \times 10^{5}$ \\
\hline $5 p^{6}$ & ${ }^{1} S$ & 0 & $5 p^{5} 6 f$ & ${ }^{3} F$ & 2 & 281 & -5.202 & $5.31 \times 10^{5}$ \\
\hline $5 p^{6}$ & ${ }^{1} S$ & 0 & $5 p^{5} 10 p$ & ${ }^{3} D$ & 2 & 270.13 & -5.287 & $4.73 \times 10^{5}$ \\
\hline $5 p^{6}$ & ${ }^{1} S$ & 0 & $5 p^{5} 8 f$ & ${ }^{3} F$ & 2 & 257.04 & -5.338 & $4.64 \times 10^{5}$ \\
\hline $5 p^{6}$ & ${ }^{1} S$ & 0 & $5 p^{5} 10 p$ & ${ }^{3} D$ & 2 & 255.26 & -5.375 & $4.32 \times 10^{5}$ \\
\hline $5 p^{5} 4 f$ & ${ }^{1} D$ & 2 & $5 p^{5} 10 h$ & ${ }^{3} G$ & 4 & 475.54 & -4.895 & $3.76 \times 10^{5}$ \\
\hline $5 p^{5} 4 f$ & ${ }^{3} F$ & 2 & $5 p^{5} 10 h$ & ${ }^{3} H$ & 4 & 406.38 & -5.044 & $3.65 \times 10^{5}$ \\
\hline $5 p^{5} 4 f$ & ${ }^{1} D$ & 2 & $5 p^{5} 10 h$ & ${ }^{3} H$ & 4 & 431.83 & -4.994 & $3.63 \times 10^{5}$ \\
\hline $5 p^{6}$ & ${ }^{1} S$ & 0 & $5 p^{5} 6 p$ & ${ }^{3} D$ & 2 & 428.67 & -5.017 & $3.49 \times 10^{5}$ \\
\hline $5 p^{5} 4 f$ & ${ }^{3} F$ & 2 & $5 p^{5} 10 h$ & ${ }^{3} G$ & 4 & 444.86 & -5.01 & $3.29 \times 10^{5}$ \\
\hline $5 p^{6}$ & ${ }^{1} S$ & 0 & $5 p^{5} 10 p$ & ${ }^{3} P$ & 2 & 269.8 & -5.461 & $3.17 \times 10^{5}$ \\
\hline $5 p^{6}$ & ${ }^{1} S$ & 0 & $5 p^{5} 7 p$ & ${ }^{3} D$ & 2 & 325.4 & -5.302 & $3.14 \times 10^{5}$ \\
\hline $5 p^{6}$ & ${ }^{1} S$ & 0 & $5 p^{5} 6 f$ & ${ }^{3} D$ & 2 & 299.44 & -5.395 & $2.99 \times 10^{5}$ \\
\hline $5 p^{6}$ & ${ }^{1} S$ & 0 & $5 p^{5} 10 f$ & ${ }^{1} D$ & 2 & 262.01 & -5.523 & $2.92 \times 10^{5}$ \\
\hline $5 p^{6}$ & ${ }^{1} S$ & 0 & $5 p^{5} 9 f$ & ${ }^{3} D$ & 2 & 266.11 & -5.524 & $2.82 \times 10^{5}$ \\
\hline $5 p^{6}$ & ${ }^{1} S$ & 0 & $5 p^{5} 10 f$ & ${ }^{3} F$ & 2 & 248.23 & -5.647 & $2.44 \times 10^{5}$ \\
\hline $5 p^{5} 4 f$ & ${ }^{1} D$ & 2 & $5 p^{5} 10 h$ & ${ }^{3} H$ & 4 & 475.44 & -5.127 & $2.21 \times 10^{5}$ \\
\hline $5 p^{5} 5 f$ & ${ }^{3} G$ & 5 & $5 p^{5} 6 h$ & ${ }^{3} I$ & 7 & 1682.75 & -4.08 & $1.96 \times 10^{5}$ \\
\hline $5 p^{6}$ & ${ }^{1} S$ & 0 & $5 p^{5} 8 p$ & ${ }^{3} D$ & 2 & 275.74 & -5.7 & $1.75 \times 10^{5}$ \\
\hline $5 p^{6}$ & ${ }^{1} S$ & 0 & $5 p^{5} 4 f$ & ${ }^{1} D$ & 2 & 571.42 & -5.08 & $1.70 \times 10^{5}$ \\
\hline $5 p^{6}$ & ${ }^{1} S$ & 0 & $5 p^{5} 8 p$ & ${ }^{3} D$ & 2 & 293.62 & -5.664 & $1.68 \times 10^{5}$ \\
\hline $5 p^{5} 5 f$ & ${ }^{1} G$ & 4 & $5 p^{5} 6 h$ & ${ }^{1} I$ & 6 & 1685.03 & -4.152 & $1.65 \times 10^{5}$ \\
\hline $5 p^{5} 4 f$ & ${ }^{3} F$ & 2 & $5 p^{5} 10 h$ & ${ }^{3} H$ & 4 & 444.77 & -5.331 & $1.57 \times 10^{5}$ \\
\hline $5 p^{5} 5 f$ & ${ }^{3} G$ & 4 & $5 p^{5} 6 h$ & ${ }^{3} I$ & 6 & 1700.59 & -4.169 & $1.56 \times 10^{5}$ \\
\hline $5 p^{6}$ & ${ }^{1} S$ & 0 & $5 p^{5} 9 p$ & ${ }^{3} D$ & 2 & 262.71 & -5.82 & $1.46 \times 10^{5}$ \\
\hline $5 p^{5} 5 f$ & ${ }^{3} G$ & 5 & $5 p^{5} 7 h$ & ${ }^{3} I$ & 7 & 1380.78 & -4.397 & $1.40 \times 10^{5}$ \\
\hline $5 p^{5} 5 f$ & ${ }^{3} F$ & 4 & $5 p^{5} 6 h$ & ${ }^{3} H$ & 6 & 1736.72 & -4.199 & $1.40 \times 10^{5}$ \\
\hline $5 p^{5} 5 f$ & ${ }^{3} G$ & 3 & $5 p^{5} 6 h$ & ${ }^{3} I$ & 5 & 1691.68 & -4.263 & $1.27 \times 10^{5}$ \\
\hline $5 p^{6}$ & ${ }^{1} S$ & 0 & $5 p^{5} 8 f$ & ${ }^{3} D$ & 2 & 272.04 & -5.863 & $1.24 \times 10^{5}$ \\
\hline $5 p^{5} 5 f$ & ${ }^{3} G$ & 4 & $5 p^{5} 7 h$ & ${ }^{3} I$ & 6 & 1393.09 & -4.458 & $1.20 \times 10^{5}$ \\
\hline $5 p^{5} 5 f$ & ${ }^{1} G$ & 4 & $5 p^{5} 7 h$ & ${ }^{1} I$ & 6 & 1382.3 & -4.473 & $1.18 \times 10^{5}$ \\
\hline $5 p^{5} 5 f$ & ${ }^{3} D$ & 3 & $5 p^{5} 6 h$ & ${ }^{3} G$ & 5 & 1701.96 & -4.303 & $1.15 \times 10^{5}$ \\
\hline $5 p^{5} 5 f$ & ${ }^{1} F$ & 3 & $5 p^{5} 6 h$ & ${ }^{1} H$ & 5 & 1730.24 & -4.292 & $1.14 \times 10^{5}$ \\
\hline $5 p^{5} 5 f$ & ${ }^{3} D$ & 3 & $5 p^{5} 6 h$ & ${ }^{3} G$ & 5 & 1705.78 & -4.331 & $1.07 \times 10^{5}$ \\
\hline $5 p^{5} 5 f$ & ${ }^{3} F$ & 4 & $5 p^{5} 7 h$ & ${ }^{3} H$ & 6 & 1418.58 & -4.526 & $9.87 \times 10^{4}$ \\
\hline $5 p^{5} 5 f$ & ${ }^{3} G$ & 3 & $5 p^{5} 7 h$ & ${ }^{3} I$ & 5 & 1387.09 & -4.55 & $9.78 \times 10^{4}$ \\
\hline $5 p^{6}$ & ${ }^{1} S$ & 0 & $5 p^{5} 8 p$ & ${ }^{3} P$ & 2 & 292.59 & -5.907 & $9.64 \times 10^{4}$ \\
\hline $5 p^{5} 5 f$ & ${ }^{3} G$ & 5 & $5 p^{5} 8 h$ & ${ }^{3} I$ & 7 & 1236.52 & -4.66 & $9.55 \times 10^{4}$ \\
\hline $5 p^{5} 5 f$ & ${ }^{3} D$ & 3 & $5 p^{5} 7 h$ & ${ }^{3} G$ & 5 & 1393.77 & -4.558 & $9.49 \times 10^{4}$ \\
\hline $5 p^{5} 6 p$ & ${ }^{3} D$ & 3 & $5 p^{5} 10 h$ & ${ }^{3} G$ & 5 & 588.73 & -5.312 & $9.37 \times 10^{4}$ \\
\hline $5 p^{6}$ & ${ }^{1} S$ & 0 & $5 p^{5} 9 p$ & ${ }^{3} D$ & 2 & 278.6 & -5.977 & $9.06 \times 10^{4}$ \\
\hline $5 p^{6}$ & ${ }^{1} S$ & 0 & $5 p^{5} 9 p$ & ${ }^{3} P$ & 2 & 278.06 & -5.979 & $9.05 \times 10^{4}$ \\
\hline $5 p^{5} 5 f$ & ${ }^{3} F$ & 2 & $5 p^{5} 6 h$ & ${ }^{3} H$ & 4 & 1707.63 & -4.41 & $8.89 \times 10^{4}$ \\
\hline $5 p^{5} 6 p$ & ${ }^{1} S$ & 0 & $5 p^{5} 9 f$ & ${ }^{3} F$ & 2 & 730.85 & -5.169 & $8.45 \times 10^{4}$ \\
\hline
\end{tabular}


TABLE VI (cont.)

\begin{tabular}{|c|c|c|c|c|c|c|c|c|}
\hline \multicolumn{3}{|c|}{ Lower Level } & \multicolumn{3}{|c|}{ Upper Level } & $\lambda[\AA]$ & $\log (g f)$ & $g A_{j i}\left[\mathrm{~s}^{-1}\right]$ \\
\hline Conf. & Term & $J$ & Conf. & Term & $J$ & HFR & HFR & HFR \\
\hline $5 p^{5} 6 p$ & ${ }^{3} P$ & 0 & $5 p^{5} 9 f$ & ${ }^{1} D$ & 2 & 759.41 & -5.155 & $8.09 \times 10^{4}$ \\
\hline $5 p^{6}$ & ${ }^{1} S$ & 0 & $5 p^{5} 4 f$ & ${ }^{3} F$ & 2 & 623.05 & -5.33 & $8.03 \times 10^{4}$ \\
\hline $5 p^{5} 4 f$ & ${ }^{1} D$ & 2 & $5 p^{5} 10 f$ & ${ }^{1} D$ & 2 & 483.9 & -5.55 & $8.03 \times 10^{4}$ \\
\hline $5 p^{5} 5 f$ & ${ }^{1} F$ & 3 & $5 p^{5} 7 h$ & ${ }^{1} H$ & 5 & 1414.24 & -4.619 & $8.02 \times 10^{4}$ \\
\hline $5 p^{5} 5 f$ & ${ }^{3} G$ & 4 & $5 p^{5} 8 h$ & ${ }^{3} I$ & 6 & 1246.68 & -4.729 & $8.01 \times 10^{4}$ \\
\hline $5 p^{5} 5 f$ & ${ }^{1} G$ & 4 & $5 p^{5} 8 h$ & ${ }^{1} I$ & 6 & 1237.73 & -4.74 & $7.92 \times 10^{4}$ \\
\hline $5 p^{5} 5 f$ & ${ }^{3} D$ & 2 & $5 p^{5} 6 h$ & ${ }^{3} G$ & 4 & 1672.12 & -4.487 & $7.78 \times 10^{4}$ \\
\hline $5 p^{5} 5 f$ & ${ }^{3} D$ & 3 & $5 p^{5} 7 h$ & ${ }^{3} G$ & 5 & 1397.43 & -4.649 & $7.67 \times 10^{4}$ \\
\hline $5 p^{5} 5 f$ & ${ }^{1} D$ & 2 & $5 p^{5} 6 h$ & ${ }^{3} H$ & 4 & 1718.72 & -4.471 & $7.64 \times 10^{4}$ \\
\hline $5 p^{5} 4 f$ & ${ }^{3} F$ & 2 & $5 p^{5} 10 f$ & ${ }^{3} F$ & 2 & 412.63 & -5.713 & $7.58 \times 10^{4}$ \\
\hline $5 p^{5} 6 p$ & ${ }^{3} D$ & 3 & $5 p^{5} 5 f$ & ${ }^{3} G$ & 5 & 1731.11 & -4.487 & $7.25 \times 10^{4}$ \\
\hline $5 p^{5} 5 f$ & ${ }^{3} F$ & 2 & $5 p^{5} 7 h$ & ${ }^{3} H$ & 4 & 1397.56 & -4.677 & $7.19 \times 10^{4}$ \\
\hline $5 p^{5} 5 f$ & ${ }^{3} G$ & 5 & $5 p^{5} 9 h$ & ${ }^{3} I$ & 7 & 1153.8 & -4.846 & $7.14 \times 10^{4}$ \\
\hline $5 p^{5} 5 f$ & ${ }^{3} F$ & 4 & $5 p^{5} 10 \mathrm{~h}$ & ${ }^{3} H$ & 6 & 1126.2 & -4.89 & $6.77 \times 10^{4}$ \\
\hline $5 p^{5} 5 f$ & ${ }^{3} G$ & 3 & $5 p^{5} 8 h$ & ${ }^{3} I$ & 5 & 1241.87 & -4.818 & $6.57 \times 10^{4}$ \\
\hline $5 p^{5} 6 p$ & ${ }^{3} D$ & 2 & $5 p^{5} 5 f$ & ${ }^{1} G$ & 4 & 1631.83 & -4.586 & $6.49 \times 10^{4}$ \\
\hline $5 p^{5} 5 f$ & ${ }^{3} D$ & 3 & $5 p^{5} 8 h$ & ${ }^{3} G$ & 5 & 1247.25 & -4.83 & $6.34 \times 10^{4}$ \\
\hline $5 p^{5} 5 f$ & ${ }^{3} D$ & 1 & $5 p^{5} 6 h$ & ${ }^{3} G$ & 3 & 1657.6 & -4.591 & $6.23 \times 10^{4}$ \\
\hline $5 p^{5} 5 f$ & ${ }^{3} F$ & 4 & $5 p^{5} 8 h$ & ${ }^{3} H$ & 6 & 1267.25 & -4.854 & $5.81 \times 10^{4}$ \\
\hline $5 p^{5} 5 f$ & ${ }^{1} G$ & 4 & $5 p^{5} 9 h$ & ${ }^{1} I$ & 6 & 1154.85 & -4.945 & $5.68 \times 10^{4}$ \\
\hline $5 p^{5} 5 f$ & ${ }^{3} D$ & 2 & $5 p^{5} 7 h$ & ${ }^{3} G$ & 4 & 1373.08 & -4.797 & $5.64 \times 10^{4}$ \\
\hline $5 p^{5} 6 p$ & ${ }^{3} D$ & 2 & $5 p^{5} 5 f$ & ${ }^{3} G$ & 4 & 1759.82 & -4.591 & $5.52 \times 10^{4}$ \\
\hline $5 p^{5} 5 f$ & ${ }^{3} G$ & 4 & $5 p^{5} 9 h$ & ${ }^{3} I$ & 6 & 1162.8 & -4.953 & $5.50 \times 10^{4}$ \\
\hline $5 p^{5} 5 f$ & ${ }^{1} D$ & 2 & $5 p^{5} 7 h$ & ${ }^{3} H$ & 4 & 1406.09 & -4.795 & $5.42 \times 10^{4}$ \\
\hline $5 p^{5} 5 f$ & ${ }^{1} F$ & 3 & $5 p^{5} 10 h$ & ${ }^{1} H$ & 5 & 1123.46 & -5.014 & $5.12 \times 10^{4}$ \\
\hline $5 p^{5} 6 p$ & ${ }^{3} D$ & 1 & $5 p^{5} 5 f$ & ${ }^{3} G$ & 3 & 1619.37 & -4.698 & $5.10 \times 10^{4}$ \\
\hline $5 p^{5} 4 f$ & ${ }^{3} D$ & 3 & $5 p^{5} 9 f$ & ${ }^{3} G$ & 5 & 442.94 & -5.829 & $5.04 \times 10^{4}$ \\
\hline $5 p^{5} 5 f$ & ${ }^{3} F$ & 2 & $5 p^{5} 8 h$ & ${ }^{3} H$ & 4 & 1250.28 & -4.953 & $4.75 \times 10^{4}$ \\
\hline $5 p^{5} 4 f$ & ${ }^{3} D$ & 1 & $5 p^{5} 9 f$ & ${ }^{3} G$ & 3 & 393.86 & -5.959 & $4.73 \times 10^{4}$ \\
\hline $5 p^{5} 5 f$ & ${ }^{1} F$ & 3 & $5 p^{5} 8 h$ & ${ }^{1} H$ & 5 & 1263.78 & -4.948 & $4.71 \times 10^{4}$ \\
\hline $5 p^{5} 6 p$ & ${ }^{3} D$ & 2 & $5 p^{5} 10 h$ & ${ }^{3} H$ & 4 & 577.06 & -5.635 & $4.64 \times 10^{4}$ \\
\hline $5 p^{5} 5 f$ & ${ }^{3} G$ & 3 & $5 p^{5} 9 h$ & ${ }^{3} I$ & 5 & 1158.61 & -5.031 & $4.63 \times 10^{4}$ \\
\hline $5 p^{5} 6 p$ & ${ }^{3} D$ & 3 & $5 p^{5} 7 f$ & ${ }^{3} G$ & 5 & 845.78 & -5.306 & $4.61 \times 10^{4}$ \\
\hline $5 p^{5} 5 f$ & ${ }^{3} D$ & 1 & $5 p^{5} 7 h$ & ${ }^{3} G$ & 3 & 1363.28 & -4.898 & $4.54 \times 10^{4}$ \\
\hline $5 p^{5} 5 f$ & ${ }^{3} D$ & 3 & $5 p^{5} 9 h$ & ${ }^{3} G$ & 5 & 1163.29 & -5.049 & $4.40 \times 10^{4}$ \\
\hline $5 p^{5} 6 p$ & ${ }^{3} D$ & 3 & $5 p^{5} 8 f$ & ${ }^{3} G$ & 5 & 763.93 & -5.429 & $4.25 \times 10^{4}$ \\
\hline $5 p^{5} 6 p$ & ${ }^{1} S$ & 0 & $5 p^{5} 9 f$ & ${ }^{1} D$ & 2 & 864.75 & -5.325 & $4.22 \times 10^{4}$ \\
\hline $5 p^{5} 4 f$ & ${ }^{3} D$ & 2 & $5 p^{5} 9 f$ & ${ }^{3} G$ & 4 & 398.02 & -6.003 & $4.18 \times 10^{4}$ \\
\hline $5 p^{5} 5 f$ & ${ }^{3} F$ & 4 & $5 p^{5} 9 h$ & ${ }^{3} H$ & 6 & 1181.11 & -5.063 & $4.14 \times 10^{4}$ \\
\hline $5 p^{5} 5 f$ & ${ }^{3} G$ & 4 & $5 p^{5} 10 h$ & ${ }^{3} I$ & 6 & 1109.39 & -5.119 & $4.12 \times 10^{4}$ \\
\hline $5 p^{5} 5 f$ & ${ }^{3} D$ & 3 & $5 p^{5} 8 h$ & ${ }^{3} G$ & 5 & 1249.93 & -5.025 & $4.03 \times 10^{4}$ \\
\hline $5 p^{5} 6 p$ & ${ }^{3} D$ & 3 & $5 p^{5} 10 h$ & ${ }^{3} G$ & 5 & 672.86 & -5.571 & $3.95 \times 10^{4}$ \\
\hline $5 p^{5} 4 f$ & ${ }^{3} F$ & 2 & $5 p^{5} 10 f$ & ${ }^{1} D$ & 2 & 452.16 & -5.922 & $3.91 \times 10^{4}$ \\
\hline $5 p^{5} 5 f$ & ${ }^{3} F$ & 2 & $5 p^{5} 10 h$ & ${ }^{3} H$ & 4 & 1112.25 & -5.15 & $3.82 \times 10^{4}$ \\
\hline $5 p^{5} 5 f$ & ${ }^{3} D$ & 2 & $5 p^{5} 8 h$ & ${ }^{3} G$ & 4 & 1230.16 & -5.065 & $3.80 \times 10^{4}$ \\
\hline $5 p^{5} 6 p$ & ${ }^{3} P$ & 2 & $5 p^{5} 5 f$ & ${ }^{3} F$ & 4 & 1759.52 & -4.757 & $3.77 \times 10^{4}$ \\
\hline $5 p^{5} 5 f$ & ${ }^{3} D$ & 3 & $5 p^{5} 9 h$ & ${ }^{3} G$ & 5 & 1166.13 & -5.129 & $3.64 \times 10^{4}$ \\
\hline $5 p^{5} 6 p$ & ${ }^{3} S$ & 1 & $5 p^{5} 9 f$ & ${ }^{3} D$ & 3 & 601.97 & -5.708 & $3.60 \times 10^{4}$ \\
\hline $5 p^{5} 6 p$ & ${ }^{3} P$ & 1 & $5 p^{5} 5 f$ & ${ }^{3} D$ & 3 & 1754.06 & -4.788 & $3.53 \times 10^{4}$ \\
\hline $5 p^{5} 6 p$ & ${ }^{3} P$ & 2 & $5 p^{5} 9 f$ & ${ }^{3} F$ & 4 & 733.66 & -5.546 & $3.52 \times 10^{4}$ \\
\hline $5 p^{5} 5 f$ & ${ }^{1} D$ & 2 & $5 p^{5} 10 h$ & ${ }^{3} H$ & 4 & 1117.99 & -5.186 & $3.48 \times 10^{4}$ \\
\hline $5 p^{5} 5 f$ & ${ }^{1} F$ & 3 & $5 p^{5} 9 h$ & ${ }^{1} H$ & 5 & 1178.1 & -5.149 & $3.41 \times 10^{4}$ \\
\hline
\end{tabular}


TABLE VI (cont.)

\begin{tabular}{|c|c|c|c|c|c|c|c|c|}
\hline \multicolumn{3}{|c|}{ Lower Level } & \multicolumn{3}{|c|}{ Upper Level } & $\lambda[\AA]$ & $\log (g f)$ & $g A_{j i}\left[\mathrm{~s}^{-1}\right]$ \\
\hline Conf. & Term & $J$ & Conf. & Term & $J$ & HFR & HFR & HFR \\
\hline $5 p^{5} 6 p$ & ${ }^{3} D$ & 2 & $5 p^{5} 7 f$ & ${ }^{3} G$ & 4 & 855.82 & -5.434 & $3.35 \times 10^{4}$ \\
\hline $5 p^{5} 6 p$ & ${ }^{3} D$ & 2 & $5 p^{5} 7 f$ & ${ }^{3} G$ & 4 & 821.88 & -5.479 & $3.28 \times 10^{4}$ \\
\hline $5 p^{5} 6 p$ & ${ }^{3} D$ & 2 & $5 p^{5} 8 f$ & ${ }^{3} G$ & 4 & 772.92 & -5.538 & $3.24 \times 10^{4}$ \\
\hline $5 p^{5} 6 p$ & ${ }^{3} D$ & 2 & $5 p^{5} 8 f$ & ${ }^{3} G$ & 4 & 744.23 & -5.582 & $3.15 \times 10^{4}$ \\
\hline $5 p^{5} 4 f$ & ${ }^{3} F$ & 4 & $5 p^{5} 10 h$ & ${ }^{3} I$ & 6 & 400.8 & -6.121 & $3.14 \times 10^{4}$ \\
\hline $5 p^{5} 4 f$ & ${ }^{1} D$ & 2 & $5 p^{5} 10 f$ & ${ }^{1} F$ & 3 & 483.92 & -5.96 & $3.13 \times 10^{4}$ \\
\hline $5 p^{5} 5 f$ & ${ }^{3} D$ & 1 & $5 p^{5} 8 h$ & ${ }^{3} G$ & 3 & 1222.28 & -5.157 & $3.11 \times 10^{4}$ \\
\hline $5 p^{5} 4 f$ & ${ }^{3} F$ & 4 & $5 p^{5} 10 h$ & ${ }^{1} I$ & 6 & 438.15 & -6.049 & $3.10 \times 10^{4}$ \\
\hline $5 p^{5} 4 f$ & ${ }^{3} G$ & 5 & $5 p^{5} 5 f$ & ${ }^{3} G$ & 5 & 686.48 & -5.664 & $3.07 \times 10^{4}$ \\
\hline $5 p^{5} 5 f$ & ${ }^{3} F$ & 2 & $5 p^{5} 9 h$ & ${ }^{3} H$ & 4 & 1165.93 & -5.206 & $3.05 \times 10^{4}$ \\
\hline $5 p^{5} 5 f$ & ${ }^{3} D$ & 3 & $5 p^{5} 10 h$ & ${ }^{3} G$ & 5 & 1109.85 & -5.254 & $3.02 \times 10^{4}$ \\
\hline $5 p^{5} 4 f$ & ${ }^{1} D$ & 2 & $5 p^{5} 10 f$ & ${ }^{3} F$ & 2 & 438.9 & -6.063 & $3.00 \times 10^{4}$ \\
\hline $5 p^{5} 6 p$ & ${ }^{3} D$ & 2 & $5 p^{5} 5 f$ & ${ }^{1} F$ & 3 & 1585.87 & -4.96 & $2.91 \times 10^{4}$ \\
\hline $5 p^{5} 5 f$ & ${ }^{1} D$ & 2 & $5 p^{5} 8 h$ & ${ }^{3} H$ & 4 & 1256.86 & -5.165 & $2.89 \times 10^{4}$ \\
\hline $5 p^{5} 6 p$ & ${ }^{3} D$ & 3 & $5 p^{5} 6 f$ & ${ }^{3} G$ & 5 & 1028.71 & -5.343 & $2.86 \times 10^{4}$ \\
\hline $5 p^{5} 5 f$ & ${ }^{3} D$ & 2 & $5 p^{5} 9 h$ & ${ }^{3} G$ & 4 & 1148.16 & -5.25 & $2.85 \times 10^{4}$ \\
\hline $5 p^{5} 5 f$ & ${ }^{1} G$ & 4 & $5 p^{5} 10 h$ & ${ }^{1} I$ & 6 & 1102.07 & -5.29 & $2.81 \times 10^{4}$ \\
\hline $5 p^{5} 4 f$ & ${ }^{3} F$ & 4 & $5 p^{5} 5 f$ & ${ }^{3} G$ & 4 & 690.55 & -5.705 & $2.76 \times 10^{4}$ \\
\hline $5 p^{5} 4 f$ & ${ }^{3} G$ & 5 & $5 p^{5} 9 h$ & ${ }^{3} I$ & 7 & 430.4 & -6.118 & $2.75 \times 10^{4}$ \\
\hline $5 p^{5} 5 f$ & ${ }^{3} G$ & 3 & $5 p^{5} 10 h$ & ${ }^{3} I$ & 5 & 1105.58 & -5.306 & $2.70 \times 10^{4}$ \\
\hline $5 p^{5} 6 p$ & ${ }^{3} P$ & 1 & $5 p^{5} 7 f$ & ${ }^{3} D$ & 3 & 854.44 & -5.532 & $2.68 \times 10^{4}$ \\
\hline $5 p^{5} 5 f$ & ${ }^{3} F$ & 4 & $5 p^{5} 6 h$ & ${ }^{3} G$ & 5 & 1738.61 & -4.922 & $2.64 \times 10^{4}$ \\
\hline $5 p^{5} 6 p$ & ${ }^{3} D$ & 1 & $5 p^{5} 7 f$ & ${ }^{3} G$ & 3 & 819.57 & -5.578 & $2.63 \times 10^{4}$ \\
\hline $5 p^{5} 6 p$ & ${ }^{3} D$ & 2 & $5 p^{5} 10 h$ & ${ }^{3} G$ & 4 & 795.68 & -5.621 & $2.52 \times 10^{4}$ \\
\hline $5 p^{5} 6 p$ & ${ }^{1} P$ & 1 & $5 p^{5} 5 f$ & ${ }^{1} F$ & 3 & 1706.83 & -4.961 & $2.50 \times 10^{4}$ \\
\hline $5 p^{5} 5 f$ & ${ }^{3} D$ & 1 & $5 p^{5} 9 h$ & ${ }^{3} G$ & 3 & 1141.29 & -5.314 & $2.48 \times 10^{4}$ \\
\hline $5 p^{5} 6 p$ & ${ }^{3} D$ & 1 & $5 p^{5} 8 f$ & ${ }^{3} G$ & 3 & 743.19 & -5.692 & $2.45 \times 10^{4}$ \\
\hline $5 p^{5} 5 f$ & ${ }^{3} D$ & 3 & $5 p^{5} 10 h$ & ${ }^{3} G$ & 5 & 1112.51 & -5.348 & $2.42 \times 10^{4}$ \\
\hline $5 p^{5} 6 p$ & ${ }^{3} D$ & 1 & $5 p^{5} 10 h$ & ${ }^{3} G$ & 3 & 763.84 & -5.679 & $2.39 \times 10^{4}$ \\
\hline $5 p^{5} 6 p$ & ${ }^{3} D$ & 2 & $5 p^{5} 6 f$ & ${ }^{1} G$ & 4 & 992.89 & -5.456 & $2.37 \times 10^{4}$ \\
\hline $5 p^{6}$ & ${ }^{1} S$ & 0 & $5 p^{5} 10 f$ & ${ }^{3} D$ & 2 & 262.11 & -6.619 & $2.33 \times 10^{4}$ \\
\hline $5 p^{5} 4 f$ & ${ }^{3} G$ & 5 & $5 p^{5} 8 h$ & ${ }^{3} I$ & 7 & 441.42 & -6.169 & $2.32 \times 10^{4}$ \\
\hline $5 p^{5} 4 f$ & ${ }^{3} G$ & 5 & $5 p^{5} 7 h$ & ${ }^{3} I$ & 7 & 458.52 & -6.139 & $2.31 \times 10^{4}$ \\
\hline $5 p^{5} 6 p$ & ${ }^{3} D$ & 3 & $5 p^{5} 10 f$ & ${ }^{3} F$ & 4 & 689.88 & -5.785 & $2.30 \times 10^{4}$ \\
\hline $5 p^{5} 4 f$ & ${ }^{3} G$ & 5 & $5 p^{5} 6 f$ & ${ }^{3} G$ & 5 & 540.21 & -6 & $2.29 \times 10^{4}$ \\
\hline $5 p^{5} 6 p$ & ${ }^{3} D$ & 3 & $5 p^{5} 5 f$ & ${ }^{3} F$ & 4 & 1671.15 & -5.023 & $2.27 \times 10^{4}$ \\
\hline $5 p^{5} 5 f$ & ${ }^{1} D$ & 2 & $5 p^{5} 9 h$ & ${ }^{3} H$ & 4 & 1172.14 & -5.331 & $2.26 \times 10^{4}$ \\
\hline $5 p^{5} 6 p$ & ${ }^{3} S$ & 1 & $5 p^{5} 5 f$ & ${ }^{3} D$ & 1 & 1600.89 & -5.061 & $2.26 \times 10^{4}$ \\
\hline $5 p^{5} 6 p$ & ${ }^{3} D$ & 2 & $5 p^{5} 9 f$ & ${ }^{3} G$ & 4 & 726.88 & -5.747 & $2.26 \times 10^{4}$ \\
\hline $5 p^{5} 6 p$ & ${ }^{3} D$ & 3 & $5 p^{5} 10 f$ & ${ }^{3} G$ & 5 & 690.63 & -5.8 & $2.22 \times 10^{4}$ \\
\hline $5 p^{5} 4 f$ & ${ }^{3} D$ & 3 & $5 p^{5} 9 f$ & ${ }^{3} F$ & 4 & 442.61 & -6.187 & $2.21 \times 10^{4}$ \\
\hline $5 p^{5} 6 p$ & ${ }^{3} P$ & 1 & $5 p^{5} 8 f$ & ${ }^{3} D$ & 3 & 772.13 & -5.711 & $2.18 \times 10^{4}$ \\
\hline $5 p^{5} 4 f$ & ${ }^{3} G$ & 3 & $5 p^{5} 5 f$ & ${ }^{3} G$ & 3 & 676.17 & -5.831 & $2.15 \times 10^{4}$ \\
\hline $5 p^{5} 5 f$ & ${ }^{3} G$ & 5 & $5 p^{5} 6 h$ & ${ }^{3} H$ & 6 & 1676.38 & -5.044 & $2.14 \times 10^{4}$ \\
\hline $5 p^{5} 5 f$ & ${ }^{3} G$ & 5 & $5 p^{5} 10 h$ & ${ }^{3} I$ & 7 & 1101.11 & -5.413 & $2.13 \times 10^{4}$ \\
\hline $5 p^{5} 6 p$ & ${ }^{3} P$ & 2 & $5 p^{5} 5 f$ & ${ }^{3} D$ & 3 & 1794.47 & -4.993 & $2.10 \times 10^{4}$ \\
\hline $5 p^{5} 6 p$ & ${ }^{3} D$ & 2 & $5 p^{5} 10 f$ & ${ }^{3} G$ & 4 & 698.3 & -5.815 & $2.09 \times 10^{4}$ \\
\hline $5 p^{5} 6 p$ & ${ }^{3} P$ & 0 & $5 p^{5} 9 f$ & ${ }^{3} F$ & 2 & 654.16 & -5.873 & $2.09 \times 10^{4}$ \\
\hline $5 p^{5} 4 f$ & ${ }^{3} G$ & 5 & $5 p^{5} 10 f$ & ${ }^{3} G$ & 5 & 429.74 & -6.239 & $2.08 \times 10^{4}$ \\
\hline $5 p^{5} 4 f$ & ${ }^{3} F$ & 2 & $5 p^{5} 9 h$ & ${ }^{3} H$ & 4 & 413.33 & -6.276 & $2.07 \times 10^{4}$ \\
\hline $5 p^{5} 4 f$ & ${ }^{3} G$ & 4 & $5 p^{5} 10 h$ & ${ }^{3} I$ & 6 & 388.55 & -6.332 & $2.06 \times 10^{4}$ \\
\hline $5 p^{5} 4 f$ & ${ }^{3} F$ & 4 & $5 p^{5} 10 h$ & ${ }^{1} I$ & 6 & 468 & -6.172 & $2.05 \times 10^{4}$ \\
\hline
\end{tabular}


TABLE VI (cont.)

\begin{tabular}{|c|c|c|c|c|c|c|c|c|}
\hline \multicolumn{3}{|c|}{ Lower Level } & \multicolumn{3}{|c|}{ Upper Level } & $\lambda[\AA]$ & $\log (g f)$ & $g A_{j i}\left[\mathrm{~s}^{-1}\right]$ \\
\hline Conf. & Term & $J$ & Conf. & Term & $J$ & HFR & HFR & HFR \\
\hline $5 p^{5} 5 f$ & ${ }^{3} D$ & 3 & $5 p^{5} 6 h$ & ${ }^{3} G$ & 4 & 1712.38 & -5.048 & $2.04 \times 10^{4}$ \\
\hline $5 p^{5} 4 f$ & ${ }^{3} F$ & 4 & $5 p^{5} 7 h$ & ${ }^{3} I$ & 6 & 461.69 & -6.19 & $2.02 \times 10^{4}$ \\
\hline $5 p^{5} 5 f$ & ${ }^{3} D$ & 3 & $5 p^{5} 10 h$ & ${ }^{3} G$ & 5 & 899.88 & -5.612 & $2.01 \times 10^{4}$ \\
\hline $5 p^{5} 4 f$ & ${ }^{3} G$ & 3 & $5 p^{5} 9 h$ & ${ }^{3} I$ & 5 & 426.98 & -6.26 & $2.01 \times 10^{4}$ \\
\hline $5 p^{5} 4 f$ & ${ }^{3} G$ & 4 & $5 p^{5} 5 f$ & ${ }^{1} G$ & 4 & 687.97 & -5.847 & $2.01 \times 10^{4}$ \\
\hline $5 p^{5} 5 f$ & ${ }^{1} F$ & 3 & $5 p^{5} 6 h$ & ${ }^{3} H$ & 4 & 1732.09 & -5.053 & $1.97 \times 10^{4}$ \\
\hline $5 p^{5} 6 p$ & ${ }^{3} P$ & 2 & $5 p^{5} 8 f$ & ${ }^{3} F$ & 4 & 779.82 & -5.748 & $1.96 \times 10^{4}$ \\
\hline $5 p^{5} 6 p$ & ${ }^{3} D$ & 2 & $5 p^{5} 10 h$ & ${ }^{3} H$ & 4 & 657.66 & -5.896 & $1.96 \times 10^{4}$ \\
\hline $5 p^{5} 5 f$ & ${ }^{1} G$ & 4 & $5 p^{5} 6 h$ & ${ }^{1} H$ & 5 & 1678.66 & -5.085 & $1.95 \times 10^{4}$ \\
\hline $5 p^{5} 4 f$ & ${ }^{3} F$ & 4 & $5 p^{5} 8 h$ & ${ }^{3} I$ & 6 & 444.4 & -6.241 & $1.94 \times 10^{4}$ \\
\hline $5 p^{5} 4 f$ & ${ }^{3} F$ & 3 & $5 p^{5} 5 f$ & ${ }^{3} D$ & 3 & 688.45 & -5.862 & $1.93 \times 10^{4}$ \\
\hline $5 p^{5} 6 p$ & ${ }^{3} D$ & 2 & $5 p^{5} 9 f$ & ${ }^{3} G$ & 4 & 701.33 & -5.85 & $1.92 \times 10^{4}$ \\
\hline $5 p^{5} 4 f$ & ${ }^{3} D$ & 2 & $5 p^{5} 9 f$ & ${ }^{3} G$ & 4 & 434.92 & -6.265 & $1.92 \times 10^{4}$ \\
\hline $5 p^{5} 6 p$ & ${ }^{3} P$ & 2 & $5 p^{5} 9 f$ & ${ }^{3} D$ & 3 & 734.6 & -5.815 & $1.89 \times 10^{4}$ \\
\hline $5 p^{5} 4 f$ & ${ }^{3} F$ & 3 & $5 p^{5} 9 h$ & ${ }^{3} G$ & 5 & 432.49 & -6.285 & $1.85 \times 10^{4}$ \\
\hline $5 p^{5} 5 f$ & ${ }^{3} F$ & 4 & $5 p^{5} 7 h$ & ${ }^{3} G$ & 5 & 1419.39 & -5.255 & $1.84 \times 10^{4}$ \\
\hline $5 p^{5} 6 p$ & ${ }^{1} S$ & 0 & $5 p^{5} 10 p$ & ${ }^{3} D$ & 2 & 761.26 & -5.797 & $1.84 \times 10^{4}$ \\
\hline $5 p^{5} 6 p$ & ${ }^{3} S$ & 1 & $5 p^{5} 5 f$ & ${ }^{3} D$ & 2 & 1587.52 & -5.171 & $1.79 \times 10^{4}$ \\
\hline $5 p^{5} 4 f$ & ${ }^{3} G$ & 4 & $5 p^{5} 10 h$ & ${ }^{1} I$ & 6 & 423.56 & -6.319 & $1.78 \times 10^{4}$ \\
\hline $5 p^{5} 5 f$ & ${ }^{3} D$ & 3 & $5 p^{5} 8 h$ & ${ }^{3} G$ & 5 & 1695.47 & -5.116 & $1.78 \times 10^{4}$ \\
\hline $5 p^{5} 4 f$ & ${ }^{3} G$ & 5 & $5 p^{5} 6 h$ & ${ }^{3} I$ & 7 & 487.57 & -6.2 & $1.77 \times 10^{4}$ \\
\hline $5 p^{5} 5 f$ & ${ }^{3} D$ & 2 & $5 p^{5} 6 h$ & ${ }^{3} G$ & 3 & 1672.19 & -5.133 & $1.76 \times 10^{4}$ \\
\hline $5 p^{5} 4 f$ & ${ }^{3} G$ & 4 & $5 p^{5} 7 h$ & ${ }^{1} I$ & 6 & 459.35 & -6.256 & $1.76 \times 10^{4}$ \\
\hline $5 p^{5} 4 f$ & ${ }^{3} F$ & 4 & $5 p^{5} 9 h$ & ${ }^{3} I$ & 6 & 433.25 & -6.309 & $1.75 \times 10^{4}$ \\
\hline $5 p^{5} 4 f$ & ${ }^{3} F$ & 2 & $5 p^{5} 10 f$ & ${ }^{3} G$ & 3 & 412.74 & -6.358 & $1.72 \times 10^{4}$ \\
\hline $5 p^{5} 6 p$ & ${ }^{3} P$ & 0 & $5 p^{5} 9 f$ & ${ }^{3} D$ & 2 & 760.92 & -5.831 & $1.70 \times 10^{4}$ \\
\hline $5 p^{5} 4 f$ & ${ }^{3} G$ & 3 & $5 p^{5} 9 h$ & ${ }^{1} H$ & 5 & 438.73 & -6.309 & $1.70 \times 10^{4}$ \\
\hline $5 p^{5} 4 f$ & ${ }^{3} G$ & 3 & $5 p^{5} 7 h$ & ${ }^{3} I$ & 5 & 454.58 & -6.28 & $1.69 \times 10^{4}$ \\
\hline $5 p^{5} 4 f$ & ${ }^{3} G$ & 3 & $5 p^{5} 8 h$ & ${ }^{3} I$ & 5 & 437.8 & -6.314 & $1.69 \times 10^{4}$ \\
\hline $5 p^{5} 6 p$ & ${ }^{3} D$ & 3 & $5 p^{5} 10 f$ & ${ }^{3} D$ & 3 & 602.09 & -6.038 & $1.69 \times 10^{4}$ \\
\hline $5 p^{5} 6 p$ & ${ }^{3} P$ & 2 & $5 p^{5} 7 f$ & ${ }^{3} F$ & 4 & 865.78 & -5.724 & $1.68 \times 10^{4}$ \\
\hline $5 p^{5} 4 f$ & ${ }^{3} G$ & 4 & $5 p^{5} 8 h$ & ${ }^{1} I$ & 6 & 442.19 & -6.318 & $1.64 \times 10^{4}$ \\
\hline $5 p^{5} 6 p$ & ${ }^{3} D$ & 2 & $5 p^{5} 10 f$ & ${ }^{1} F$ & 3 & 674 & -5.959 & $1.61 \times 10^{4}$ \\
\hline $5 p^{5} 4 f$ & ${ }^{3} F$ & 4 & $5 p^{5} 7 h$ & ${ }^{3} H$ & 6 & 476.24 & -6.263 & $1.61 \times 10^{4}$ \\
\hline $5 p^{5} 4 f$ & ${ }^{3} G$ & 5 & $5 p^{5} 7 f$ & ${ }^{3} G$ & 5 & 485.11 & -6.25 & $1.59 \times 10^{4}$ \\
\hline $5 p^{5} 5 f$ & ${ }^{1} D$ & 2 & $5 p^{5} 6 h$ & ${ }^{3} G$ & 4 & 1725.4 & -5.151 & $1.58 \times 10^{4}$ \\
\hline $5 p^{5} 4 f$ & ${ }^{3} F$ & 3 & $5 p^{5} 7 h$ & ${ }^{3} G$ & 5 & 460.83 & -6.298 & $1.58 \times 10^{4}$ \\
\hline $5 p^{5} 4 f$ & ${ }^{3} F$ & 4 & $5 p^{5} 9 h$ & ${ }^{3} H$ & 6 & 446.13 & -6.327 & $1.58 \times 10^{4}$ \\
\hline $5 p^{5} 4 f$ & ${ }^{3} F$ & 3 & $5 p^{5} 8 h$ & ${ }^{3} G$ & 5 & 443.6 & -6.333 & $1.58 \times 10^{4}$ \\
\hline $5 p^{5} 4 f$ & ${ }^{3} F$ & 2 & $5 p^{5} 10 f$ & ${ }^{3} D$ & 3 & 412.7 & -6.397 & $1.57 \times 10^{4}$ \\
\hline $5 p^{5} 5 f$ & ${ }^{3} G$ & 5 & $5 p^{5} 7 h$ & ${ }^{3} H$ & 6 & 1378.06 & -5.354 & $1.55 \times 10^{4}$ \\
\hline $5 p^{5} 6 p$ & ${ }^{3} D$ & 1 & $5 p^{5} 10 f$ & ${ }^{3} G$ & 3 & 673.79 & -5.976 & $1.55 \times 10^{4}$ \\
\hline $5 p^{5} 6 f$ & ${ }^{3} F$ & 4 & $5 p^{5} 10 h$ & ${ }^{3} H$ & 6 & 1982.67 & -5.045 & $1.53 \times 10^{4}$ \\
\hline $5 p^{5} 6 p$ & ${ }^{3} D$ & 2 & $5 p^{5} 10 f$ & ${ }^{3} G$ & 4 & 674.56 & -5.987 & $1.51 \times 10^{4}$ \\
\hline $5 p^{5} 4 f$ & ${ }^{3} G$ & 3 & $5 p^{5} 10 f$ & ${ }^{3} G$ & 3 & 426.34 & -6.388 & $1.50 \times 10^{4}$ \\
\hline $5 p^{5} 5 f$ & ${ }^{3} D$ & 3 & $5 p^{5} 7 h$ & ${ }^{3} G$ & 4 & 1400.12 & -5.371 & $1.45 \times 10^{4}$ \\
\hline $5 p^{5} 6 p$ & ${ }^{1} S$ & 0 & $5 p^{5} 5 f$ & ${ }^{3} F$ & 2 & 1777.31 & -5.167 & $1.44 \times 10^{4}$ \\
\hline $5 p^{5} 4 f$ & ${ }^{3} G$ & 4 & $5 p^{5} 6 f$ & ${ }^{1} G$ & 4 & 541.16 & -6.2 & $1.44 \times 10^{4}$ \\
\hline $5 p^{5} 5 f$ & ${ }^{1} G$ & 4 & $5 p^{5} 7 h$ & ${ }^{1} H$ & 5 & 1379.59 & -5.397 & $1.40 \times 10^{4}$ \\
\hline $5 p^{5} 4 f$ & ${ }^{3} G$ & 5 & $5 p^{5} 10 h$ & ${ }^{3} G$ & 5 & 387.95 & -6.5 & $1.40 \times 10^{4}$ \\
\hline $5 p^{5} 6 p$ & ${ }^{3} S$ & 1 & $5 p^{5} 8 p$ & ${ }^{3} D$ & 3 & 907.93 & -5.762 & $1.40 \times 10^{4}$ \\
\hline $5 p^{5} 4 f$ & ${ }^{3} G$ & 4 & $5 p^{5} 6 h$ & ${ }^{1} I$ & 6 & 488.52 & -6.305 & $1.38 \times 10^{4}$ \\
\hline
\end{tabular}


TABLE VI (cont.)

\begin{tabular}{|c|c|c|c|c|c|c|c|c|}
\hline \multicolumn{3}{|c|}{ Lower Level } & \multicolumn{3}{|c|}{ Upper Level } & $\lambda[\AA]$ & $\log (g f)$ & $g A_{j i}\left[\mathrm{~s}^{-1}\right]$ \\
\hline Conf. & Term & $J$ & Conf. & Term & $J$ & HFR & HFR & HFR \\
\hline $5 p^{5} 6 p$ & ${ }^{3} P$ & 1 & $5 p^{5} 10 f$ & ${ }^{3} D$ & 3 & 697.63 & -5.998 & $1.38 \times 10^{4}$ \\
\hline $5 p^{5} 6 f$ & ${ }^{3} G$ & 5 & $5 p^{5} 9 h$ & ${ }^{3} I$ & 7 & 2117.42 & -5.034 & $1.38 \times 10^{4}$ \\
\hline $5 p^{5} 5 f$ & ${ }^{1} F$ & 3 & $5 p^{5} 7 h$ & ${ }^{3} H$ & 4 & 1415.03 & -5.387 & $1.37 \times 10^{4}$ \\
\hline $5 p^{5} 5 f$ & ${ }^{3} G$ & 5 & $5 p^{5} 10 h$ & ${ }^{3} G$ & 5 & 892.13 & -5.788 & $1.36 \times 10^{4}$ \\
\hline $5 p^{5} 4 f$ & ${ }^{3} G$ & 4 & $5 p^{5} 9 h$ & ${ }^{1} I$ & 6 & 431.14 & -6.422 & $1.36 \times 10^{4}$ \\
\hline $5 p^{5} 4 f$ & ${ }^{3} F$ & 3 & $5 p^{5} 10 f$ & ${ }^{3} D$ & 3 & 431.8 & -6.421 & $1.36 \times 10^{4}$ \\
\hline $5 p^{5} 4 f$ & ${ }^{3} F$ & 4 & $5 p^{5} 8 h$ & ${ }^{3} H$ & 6 & 457.88 & -6.373 & $1.35 \times 10^{4}$ \\
\hline $5 p^{5} 6 p$ & ${ }^{1} P$ & 1 & $5 p^{5} 9 f$ & ${ }^{1} F$ & 3 & 723.56 & -5.976 & $1.35 \times 10^{4}$ \\
\hline $5 p^{5} 4 f$ & ${ }^{3} G$ & 3 & $5 p^{5} 7 h$ & ${ }^{1} H$ & 5 & 467.82 & -6.357 & $1.34 \times 10^{4}$ \\
\hline $5 p^{5} 4 f$ & ${ }^{3} F$ & 4 & $5 p^{5} 6 h$ & ${ }^{3} I$ & 6 & 491.12 & -6.317 & $1.33 \times 10^{4}$ \\
\hline $5 p^{5} 4 f$ & ${ }^{3} F$ & 3 & $5 p^{5} 9 f$ & ${ }^{3} G$ & 5 & 488.66 & -6.322 & $1.33 \times 10^{4}$ \\
\hline $5 p^{5} 5 f$ & ${ }^{3} F$ & 2 & $5 p^{5} 8 h$ & ${ }^{3} H$ & 4 & 1701.1 & -5.241 & $1.32 \times 10^{4}$ \\
\hline $5 p^{5} 6 f$ & ${ }^{3} G$ & 5 & $5 p^{5} 8 h$ & ${ }^{3} I$ & 7 & 2413.77 & -4.939 & $1.32 \times 10^{4}$ \\
\hline $5 p^{5} 6 p$ & ${ }^{1} P$ & 1 & $5 p^{5} 5 f$ & ${ }^{1} D$ & 2 & 1720.02 & -5.234 & $1.32 \times 10^{4}$ \\
\hline $5 p^{5} 6 p$ & ${ }^{3} D$ & 1 & $5 p^{5} 5 f$ & ${ }^{3} F$ & 2 & 1605.23 & -5.294 & $1.32 \times 10^{4}$ \\
\hline $5 p^{5} 4 f$ & ${ }^{3} D$ & 3 & $5 p^{5} 9 h$ & ${ }^{3} G$ & 5 & 432.7 & -6.436 & $1.31 \times 10^{4}$ \\
\hline $5 p^{5} 4 f$ & ${ }^{3} F$ & 4 & $5 p^{5} 7 f$ & ${ }^{3} G$ & 4 & 488.2 & -6.333 & $1.30 \times 10^{4}$ \\
\hline $5 p^{5} 6 p$ & ${ }^{3} P$ & 2 & $5 p^{5} 6 f$ & ${ }^{3} F$ & 4 & 1050.52 & -5.669 & $1.30 \times 10^{4}$ \\
\hline $5 p^{5} 6 p$ & ${ }^{3} P$ & 0 & $5 p^{5} 10 p$ & ${ }^{3} D$ & 2 & 794.72 & -5.918 & $1.27 \times 10^{4}$ \\
\hline $5 p^{5} 5 f$ & ${ }^{3} D$ & 2 & $5 p^{5} 7 h$ & ${ }^{3} G$ & 3 & 1373.13 & -5.445 & $1.27 \times 10^{4}$ \\
\hline $5 p^{5} 5 f$ & ${ }^{3} D$ & 3 & $5 p^{5} 6 h$ & ${ }^{3} G$ & 5 & 1253.46 & -5.528 & $1.26 \times 10^{4}$ \\
\hline $5 p^{5} 6 p$ & ${ }^{3} S$ & 1 & $5 p^{5} 7 f$ & ${ }^{3} D$ & 1 & 809.06 & -5.911 & $1.25 \times 10^{4}$ \\
\hline $5 p^{5} 4 f$ & ${ }^{3} F$ & 4 & $5 p^{5} 6 h$ & ${ }^{3} H$ & 6 & 507.45 & -6.317 & $1.25 \times 10^{4}$ \\
\hline $5 p^{5} 4 f$ & ${ }^{3} F$ & 4 & $5 p^{5} 5 f$ & ${ }^{3} F$ & 4 & 716.92 & -6.019 & $1.24 \times 10^{4}$ \\
\hline $5 p^{5} 4 f$ & ${ }^{3} F$ & 4 & $5 p^{5} 6 f$ & ${ }^{3} G$ & 4 & 542.88 & -6.263 & $1.24 \times 10^{4}$ \\
\hline $5 p^{5} 6 p$ & ${ }^{3} D$ & 2 & $5 p^{5} 8 f$ & ${ }^{1} F$ & 3 & 742.76 & -5.994 & $1.23 \times 10^{4}$ \\
\hline $5 p^{5} 4 f$ & ${ }^{3} D$ & 3 & $5 p^{5} 5 f$ & ${ }^{3} F$ & 4 & 682.77 & -6.074 & $1.21 \times 10^{4}$ \\
\hline $5 p^{5} 6 p$ & ${ }^{1} P$ & 1 & $5 p^{5} 8 f$ & ${ }^{1} F$ & 3 & 768.26 & -5.971 & $1.21 \times 10^{4}$ \\
\hline $5 p^{5} 4 f$ & ${ }^{1} D$ & 2 & $5 p^{5} 10 f$ & ${ }^{3} D$ & 2 & 484.21 & -6.372 & $1.21 \times 10^{4}$ \\
\hline $5 p^{5} 5 f$ & ${ }^{3} G$ & 4 & $5 p^{5} 8 h$ & ${ }^{3} H$ & 6 & 1693.98 & -5.286 & $1.20 \times 10^{4}$ \\
\hline $5 p^{5} 6 p$ & ${ }^{3} P$ & 2 & $5 p^{5} 10 f$ & ${ }^{3} F$ & 4 & 704.48 & -6.057 & $1.18 \times 10^{4}$ \\
\hline $5 p^{5} 6 f$ & ${ }^{1} F$ & 3 & $5 p^{5} 10 h$ & ${ }^{1} H$ & 5 & 1978.54 & -5.16 & $1.18 \times 10^{4}$ \\
\hline $5 p^{5} 4 f$ & ${ }^{3} G$ & 5 & $5 p^{5} 8 f$ & ${ }^{3} G$ & 5 & 457.02 & -6.435 & $1.17 \times 10^{4}$ \\
\hline $5 p^{5} 4 f$ & ${ }^{3} F$ & 3 & $5 p^{5} 7 f$ & ${ }^{3} D$ & 3 & 487.14 & -6.38 & $1.17 \times 10^{4}$ \\
\hline $5 p^{5} 6 p$ & ${ }^{3} P$ & 2 & $5 p^{5} 8 f$ & ${ }^{3} D$ & 3 & 780.85 & -5.974 & $1.16 \times 10^{4}$ \\
\hline $5 p^{5} 7 p$ & ${ }^{3} D$ & 3 & $5 p^{5} 10 h$ & ${ }^{3} G$ & 5 & 1018.87 & -5.749 & $1.15 \times 10^{4}$ \\
\hline $5 p^{5} 4 f$ & ${ }^{3} D$ & 2 & $5 p^{5} 9 f$ & ${ }^{1} F$ & 3 & 434.75 & -6.489 & $1.14 \times 10^{4}$ \\
\hline $5 p^{5} 6 p$ & ${ }^{3} D$ & 3 & $5 p^{5} 8 f$ & ${ }^{3} F$ & 4 & 761.97 & -6.007 & $1.13 \times 10^{4}$ \\
\hline $5 p^{5} 6 f$ & ${ }^{1} G$ & 4 & $5 p^{5} 9 h$ & ${ }^{1} I$ & 6 & 2120.6 & -5.12 & $1.13 \times 10^{4}$ \\
\hline $5 p^{5} 6 p$ & ${ }^{3} S$ & 1 & $5 p^{5} 8 f$ & ${ }^{3} D$ & 1 & 732.99 & -6.044 & $1.12 \times 10^{4}$ \\
\hline $5 p^{5} 6 f$ & ${ }^{1} G$ & 4 & $5 p^{5} 8 h$ & ${ }^{1} I$ & 6 & 2417.9 & -5.009 & $1.12 \times 10^{4}$ \\
\hline $5 p^{5} 4 f$ & ${ }^{3} D$ & 3 & $5 p^{5} 7 h$ & ${ }^{3} G$ & 5 & 461.01 & -6.449 & $1.12 \times 10^{4}$ \\
\hline $5 p^{5} 6 p$ & ${ }^{3} S$ & 1 & $5 p^{5} 5 f$ & ${ }^{3} D$ & 3 & 1552.85 & -5.395 & $1.11 \times 10^{4}$ \\
\hline $5 p^{5} 5 f$ & ${ }^{3} F$ & 4 & $5 p^{5} 8 h$ & ${ }^{3} G$ & 5 & 1267.47 & -5.572 & $1.11 \times 10^{4}$ \\
\hline $5 p^{5} 4 f$ & ${ }^{1} D$ & 2 & $5 p^{5} 5 f$ & ${ }^{3} F$ & 2 & 705.9 & -6.085 & $1.10 \times 10^{4}$ \\
\hline $5 p^{5} 6 p$ & ${ }^{1} P$ & 1 & $5 p^{5} 9 f$ & ${ }^{1} D$ & 2 & 723.1 & -6.067 & $1.09 \times 10^{4}$ \\
\hline $5 p^{5} 4 f$ & ${ }^{3} F$ & 2 & $5 p^{5} 10 f$ & ${ }^{1} F$ & 3 & 452.18 & -6.478 & $1.09 \times 10^{4}$ \\
\hline $5 p^{5} 4 f$ & ${ }^{3} G$ & 3 & $5 p^{5} 8 h$ & ${ }^{1} H$ & 5 & 450.09 & -6.485 & $1.08 \times 10^{4}$ \\
\hline $5 p^{5} 5 f$ & ${ }^{1} D$ & 2 & $5 p^{5} 7 h$ & ${ }^{3} G$ & 4 & 1408.81 & -5.496 & $1.07 \times 10^{4}$ \\
\hline $5 p^{5} 6 f$ & ${ }^{3} G$ & 5 & $5 p^{5} 6 h$ & ${ }^{3} I$ & 7 & 5004.16 & -4.4 & $1.06 \times 10^{4}$ \\
\hline $5 p^{5} 4 f$ & ${ }^{3} F$ & 4 & $5 p^{5} 10 h$ & ${ }^{3} H$ & 6 & 467.89 & -6.458 & $1.06 \times 10^{4}$ \\
\hline $5 p^{5} 6 p$ & ${ }^{3} D$ & 3 & $5 p^{5} 8 p$ & ${ }^{3} S$ & 1 & 965.7 & -5.833 & $1.05 \times 10^{4}$ \\
\hline
\end{tabular}


TABLE VI (cont.)

\begin{tabular}{|c|c|c|c|c|c|c|c|c|}
\hline \multicolumn{3}{|c|}{ Lower Level } & \multicolumn{3}{|c|}{ Upper Level } & $\lambda[\AA]$ & $\log (g f)$ & $g A_{j i}\left[\mathrm{~s}^{-1}\right]$ \\
\hline Conf. & Term & $J$ & Conf. & Term & $J$ & HFR & HFR & HFR \\
\hline $5 p^{5} 6 p$ & ${ }^{3} S$ & 1 & $5 p^{5} 10 f$ & ${ }^{3} D$ & 2 & 664.56 & -6.159 & $1.05 \times 10^{4}$ \\
\hline $5 p^{5} 4 f$ & ${ }^{3} G$ & 5 & $5 p^{5} 9 f$ & ${ }^{3} G$ & 5 & 440.44 & -6.519 & $1.04 \times 10^{4}$ \\
\hline $5 p^{5} 5 f$ & ${ }^{1} D$ & 2 & $5 p^{5} 10 h$ & ${ }^{3} G$ & 4 & 1118.56 & -5.71 & $1.04 \times 10^{4}$ \\
\hline $5 p^{5} 4 f$ & ${ }^{3} F$ & 2 & $5 p^{5} 10 f$ & ${ }^{3} D$ & 3 & 452.29 & -6.497 & $1.04 \times 10^{4}$ \\
\hline $5 p^{5} 6 p$ & ${ }^{3} P$ & 2 & $5 p^{5} 10 f$ & ${ }^{3} D$ & 3 & 704.85 & -6.112 & $1.04 \times 10^{4}$ \\
\hline $5 p^{5} 4 f$ & ${ }^{3} D$ & 1 & $5 p^{5} 10 p$ & ${ }^{3} P$ & 1 & 402.43 & -6.598 & $1.04 \times 10^{4}$ \\
\hline $5 p^{5} 7 p$ & ${ }^{3} D$ & 3 & $5 p^{5} 6 f$ & ${ }^{3} G$ & 5 & 3921.59 & -4.623 & $1.03 \times 10^{4}$ \\
\hline $5 p^{5} 5 f$ & ${ }^{1} G$ & 4 & $5 p^{5} 10 h$ & ${ }^{3} H$ & 4 & 892.76 & -5.908 & $1.03 \times 10^{4}$ \\
\hline $5 p^{5} 6 p$ & ${ }^{3} S$ & 1 & $5 p^{5} 7 f$ & ${ }^{3} D$ & 2 & 808.77 & -5.996 & $1.03 \times 10^{4}$ \\
\hline $5 p^{5} 6 p$ & ${ }^{3} P$ & 1 & $5 p^{5} 9 f$ & ${ }^{3} D$ & 3 & 726.16 & -6.09 & $1.03 \times 10^{4}$ \\
\hline $5 p^{5} 4 f$ & ${ }^{3} G$ & 3 & $5 p^{5} 6 h$ & ${ }^{3} I$ & 5 & 483.08 & -6.445 & $1.03 \times 10^{4}$ \\
\hline $5 p^{5} 4 f$ & ${ }^{3} G$ & 3 & $5 p^{5} 6 h$ & ${ }^{1} H$ & 5 & 497.9 & -6.419 & $1.03 \times 10^{4}$ \\
\hline $5 p^{5} 6 p$ & ${ }^{3} S$ & 1 & $5 p^{5} 8 f$ & ${ }^{3} D$ & 2 & 732.41 & -6.086 & $1.02 \times 10^{4}$ \\
\hline $5 p^{5} 4 f$ & ${ }^{3} G$ & 4 & $5 p^{5} 7 f$ & ${ }^{3} G$ & 4 & 486.04 & -6.447 & $1.01 \times 10^{4}$ \\
\hline $5 p^{5} 4 f$ & ${ }^{3} G$ & 3 & $5 p^{5} 7 f$ & ${ }^{3} G$ & 3 & 480.41 & -6.459 & $1.01 \times 10^{4}$ \\
\hline $5 p^{5} 4 f$ & ${ }^{3} G$ & 3 & $5 p^{5} 5 f$ & ${ }^{1} F$ & 3 & 699.06 & -6.133 & $1.00 \times 10^{4}$ \\
\hline $5 p^{5} 4 f$ & ${ }^{3} F$ & 3 & $5 p^{5} 6 f$ & ${ }^{3} D$ & 3 & 541.49 & -6.356 & $1.00 \times 10^{4}$ \\
\hline $5 p^{5} 6 p$ & ${ }^{3} D$ & 2 & $5 p^{5} 6 f$ & ${ }^{3} G$ & 4 & 1039.35 & -5.79 & $1.00 \times 10^{4}$ \\
\hline $5 p^{5} 6 s$ & ${ }^{3} P^{o}$ & 1 & $5 p^{5} 6 d$ & ${ }^{3} D^{o}$ & 1 & 1119.26 & -4.95 & $5.97 \times 10^{4}$ \\
\hline $5 p^{5} 6 s$ & ${ }^{3} P^{o}$ & 2 & $5 p^{5} 7 d$ & ${ }^{3} P^{o}$ & 1 & 757.69 & -5.897 & $1.47 \times 10^{4}$ \\
\hline $5 p^{5} 5 d$ & ${ }^{3} P^{o}$ & 2 & $5 p^{5} 7 d$ & ${ }^{3} F^{o}$ & 4 & 595.64 & -5.81 & $2.91 \times 10^{4}$ \\
\hline $5 p^{5} 5 d$ & ${ }^{3} F^{o}$ & 4 & $5 p^{5} 7 d$ & ${ }^{3} F^{o}$ & 4 & 598.75 & -5.602 & $4.65 \times 10^{4}$ \\
\hline $5 p^{5} 6 s$ & ${ }^{3} P^{o}$ & 2 & $5 p^{5} 7 d$ & ${ }^{3} F^{o}$ & 4 & 755.22 & -5.432 & $4.32 \times 10^{4}$ \\
\hline $5 p^{5} 7 s$ & ${ }^{3} P^{o}$ & 2 & $5 p^{5} 7 d$ & ${ }^{3} F^{o}$ & 4 & 2586.7 & -4.672 & $2.12 \times 10^{4}$ \\
\hline $5 p^{5} 5 d$ & ${ }^{3} F^{o}$ & 3 & $5 p^{5} 7 d$ & ${ }^{3} F^{o}$ & 3 & 603.14 & -5.997 & $1.85 \times 10^{4}$ \\
\hline $5 p^{5} 5 d$ & ${ }^{3} D^{o}$ & 3 & $5 p^{5} 7 d$ & ${ }^{3} F^{o}$ & 3 & 635.52 & -6.161 & $1.14 \times 10^{4}$ \\
\hline $5 p^{5} 5 d$ & ${ }^{3} D^{o}$ & 1 & $5 p^{5} 7 d$ & ${ }^{3} F^{o}$ & 3 & 667.05 & -5.879 & $1.98 \times 10^{4}$ \\
\hline $5 p^{5} 6 s$ & ${ }^{3} P^{o}$ & 2 & $5 p^{5} 7 d$ & ${ }^{3} F^{o}$ & 3 & 755.07 & -6.018 & $1.12 \times 10^{4}$ \\
\hline $5 p^{5} 6 s$ & ${ }^{1} P^{o}$ & 1 & $5 p^{5} 7 d$ & ${ }^{3} F^{o}$ & 3 & 765.76 & -5.73 & $2.12 \times 10^{4}$ \\
\hline $5 p^{5} 5 d$ & ${ }^{1} P^{o}$ & 1 & $5 p^{5} 7 d$ & ${ }^{3} F^{o}$ & 3 & 870.94 & -5.851 & $1.24 \times 10^{4}$ \\
\hline $5 p^{5} 5 d$ & ${ }^{3} P^{o}$ & 0 & $5 p^{5} 7 d$ & ${ }^{3} P^{o}$ & 2 & 571.98 & -5.264 & $1.11 \times 10^{5}$ \\
\hline $5 p^{5} 5 d$ & ${ }^{3} P^{o}$ & 1 & $5 p^{5} 7 d$ & ${ }^{3} P^{o}$ & 2 & 579.56 & -5.571 & $5.34 \times 10^{4}$ \\
\hline $5 p^{5} 5 d$ & ${ }^{3} P^{o}$ & 2 & $5 p^{5} 7 d$ & ${ }^{3} P^{o}$ & 2 & 594.98 & -6.018 & $1.81 \times 10^{4}$ \\
\hline $5 p^{5} 5 d$ & ${ }^{3} D^{o}$ & 3 & $5 p^{5} 7 d$ & ${ }^{3} P^{o}$ & 2 & 634.88 & -6.172 & $1.11 \times 10^{4}$ \\
\hline $5 p^{5} 6 s$ & ${ }^{3} P^{o}$ & 2 & $5 p^{5} 7 d$ & ${ }^{3} P^{o}$ & 2 & 754.17 & -5.895 & $1.50 \times 10^{4}$ \\
\hline $5 p^{5} 6 d$ & ${ }^{3} P^{o}$ & 1 & $5 p^{5} 7 d$ & ${ }^{3} P^{o}$ & 2 & 2175.36 & -5.066 & $1.21 \times 10^{4}$ \\
\hline $5 p^{5} 5 d$ & ${ }^{3} P^{o}$ & 0 & $5 p^{5} 5 g$ & ${ }^{3} F^{o}$ & 2 & 571.88 & -5.457 & $7.12 \times 10^{4}$ \\
\hline $5 p^{5} 5 d$ & ${ }^{3} P^{o}$ & 1 & $5 p^{5} 5 g$ & ${ }^{3} F^{o}$ & 2 & 579.45 & -5.094 & $1.60 \times 10^{5}$ \\
\hline $5 p^{5} 5 d$ & ${ }^{3} P^{o}$ & 2 & $5 p^{5} 5 g$ & ${ }^{3} F^{o}$ & 2 & 594.87 & -5.829 & $2.80 \times 10^{4}$ \\
\hline $5 p^{5} 5 d$ & ${ }^{3} F^{o}$ & 2 & $5 p^{5} 5 g$ & ${ }^{3} F^{o}$ & 2 & 614.45 & -6.087 & $1.44 \times 10^{4}$ \\
\hline $5 p^{5} 6 d$ & ${ }^{3} P^{o}$ & 0 & $5 p^{5} 5 g$ & ${ }^{3} F^{o}$ & 2 & 2139.66 & -5.069 & $1.24 \times 10^{4}$ \\
\hline $5 p^{5} 5 d$ & ${ }^{3} P^{o}$ & 1 & $5 p^{5} 5 g$ & ${ }^{3} F^{o}$ & 3 & 579.21 & -4.991 & $2.03 \times 10^{5}$ \\
\hline $5 p^{5} 5 d$ & ${ }^{3} P^{o}$ & 2 & $5 p^{5} 5 g$ & ${ }^{3} F^{o}$ & 3 & 594.61 & -5.094 & $1.52 \times 10^{5}$ \\
\hline $5 p^{5} 5 d$ & ${ }^{3} F^{o}$ & 3 & $5 p^{5} 5 g$ & ${ }^{3} F^{o}$ & 3 & 602.18 & -6.196 & $1.17 \times 10^{4}$ \\
\hline $5 p^{5} 5 d$ & ${ }^{3} D^{o}$ & 3 & $5 p^{5} 5 g$ & ${ }^{3} F^{o}$ & 3 & 634.46 & -5.844 & $2.37 \times 10^{4}$ \\
\hline $5 p^{5} 5 d$ & ${ }^{3} D^{o}$ & 1 & $5 p^{5} 5 g$ & ${ }^{3} F^{o}$ & 3 & 665.88 & -5.395 & $6.05 \times 10^{4}$ \\
\hline $5 p^{5} 5 d$ & ${ }^{3} D^{o}$ & 2 & $5 p^{5} 5 g$ & ${ }^{3} F^{o}$ & 3 & 701.09 & -6.067 & $1.16 \times 10^{4}$ \\
\hline $5 p^{5} 5 d$ & ${ }^{1} P^{o}$ & 1 & $5 p^{5} 5 g$ & ${ }^{3} F^{o}$ & 3 & 868.94 & -5.084 & $7.29 \times 10^{4}$ \\
\hline $5 p^{5} 6 d$ & ${ }^{3} P^{o}$ & 1 & $5 p^{5} 5 g$ & ${ }^{3} F^{o}$ & 3 & 2170.43 & -5.092 & $1.15 \times 10^{4}$ \\
\hline $5 p^{5} 6 d$ & ${ }^{3} P^{o}$ & 2 & $5 p^{5} 5 g$ & ${ }^{3} F^{o}$ & 3 & 2228.26 & -4.922 & $1.61 \times 10^{4}$ \\
\hline $5 p^{5} 5 d$ & ${ }^{3} F^{o}$ & 4 & $5 p^{5} 5 g$ & ${ }^{3} H^{o}$ & 6 & 597.13 & -4.222 & $1.12 \times 10^{6}$ \\
\hline $5 p^{5} 6 d$ & ${ }^{3} F^{o}$ & 4 & $5 p^{5} 5 g$ & ${ }^{3} H^{o}$ & 6 & 2204.1 & -4.19 & $8.87 \times 10^{4}$ \\
\hline
\end{tabular}


TABLE VI (cont.)

\begin{tabular}{|c|c|c|c|c|c|c|c|c|}
\hline \multicolumn{3}{|c|}{ Lower Level } & \multicolumn{3}{|c|}{ Upper Level } & $\lambda[\AA]$ & $\overline{\log (g f)}$ & $g A_{j i}\left[\mathrm{~s}^{-1}\right]$ \\
\hline Conf. & Term & $J$ & Conf. & Term & $J$ & HFR & HFR & HFR \\
\hline $5 p^{5} 5 d$ & ${ }^{3} F^{o}$ & 4 & $5 p^{5} 5 g$ & ${ }^{1} H^{o}$ & 5 & 597.01 & -5.589 & $4.82 \times 10^{4}$ \\
\hline $5 p^{5} 5 d$ & ${ }^{3} F^{o}$ & 3 & $5 p^{5} 5 g$ & ${ }^{1} H^{o}$ & 5 & 601.46 & -4.405 & $7.26 \times 10^{5}$ \\
\hline $5 p^{5} 5 d$ & ${ }^{3} D^{o}$ & 3 & $5 p^{5} 5 g$ & ${ }^{1} H^{o}$ & 5 & 633.65 & -5.022 & $1.58 \times 10^{5}$ \\
\hline $5 p^{5} 5 d$ & ${ }^{1} F^{o}$ & 3 & $5 p^{5} 5 g$ & ${ }^{1} H^{o}$ & 5 & 712.22 & -5.732 & $2.44 \times 10^{4}$ \\
\hline $5 p^{5} 6 d$ & ${ }^{3} F^{o}$ & 3 & $5 p^{5} 5 g$ & ${ }^{1} H^{o}$ & 5 & 2217.52 & -4.305 & $6.72 \times 10^{4}$ \\
\hline $5 p^{5} 6 s$ & ${ }^{1} P^{o}$ & 1 & $5 p^{5} 7 d$ & ${ }^{1} D^{o}$ & 2 & 761.98 & -5.702 & $2.28 \times 10^{4}$ \\
\hline $5 p^{5} 7 s$ & ${ }^{1} P^{o}$ & 1 & $5 p^{5} 7 d$ & ${ }^{1} D^{o}$ & 2 & 2589.46 & -4.977 & $1.05 \times 10^{4}$ \\
\hline $5 p^{5} 5 d$ & ${ }^{3} P^{o}$ & 1 & $5 p^{5} 7 d$ & ${ }^{3} D^{o}$ & 3 & 577.08 & -5.783 & $3.30 \times 10^{4}$ \\
\hline $5 p^{5} 5 d$ & ${ }^{3} P^{o}$ & 2 & $5 p^{5} 7 d$ & ${ }^{3} D^{\circ}$ & 3 & 592.36 & -5.354 & $8.41 \times 10^{4}$ \\
\hline $5 p^{5} 5 d$ & ${ }^{3} F^{o}$ & 3 & $5 p^{5} 7 d$ & ${ }^{3} D^{o}$ & 3 & 599.88 & -5.977 & $1.95 \times 10^{4}$ \\
\hline $5 p^{5} 5 d$ & ${ }^{3} F^{o}$ & 2 & $5 p^{5} 7 d$ & ${ }^{3} D^{o}$ & 3 & 611.77 & -5.566 & $4.84 \times 10^{4}$ \\
\hline $5 p^{5} 5 d$ & ${ }^{3} D^{\circ}$ & 1 & $5 p^{5} 7 d$ & ${ }^{3} D^{\circ}$ & 3 & 663.06 & -5.576 & $4.03 \times 10^{4}$ \\
\hline $5 p^{5} 6 s$ & ${ }^{3} P^{o}$ & 2 & $5 p^{5} 7 d$ & ${ }^{3} D^{o}$ & 3 & 749.96 & -5.796 & $1.90 \times 10^{4}$ \\
\hline $5 p^{5} 5 d$ & ${ }^{1} P^{o}$ & 1 & $5 p^{5} 7 d$ & ${ }^{3} D^{o}$ & 3 & 864.15 & -5.73 & $1.66 \times 10^{4}$ \\
\hline $5 p^{5} 6 d$ & ${ }^{3} P^{o}$ & 1 & $5 p^{5} 7 d$ & ${ }^{3} D^{o}$ & 3 & 2140.76 & -5.15 & $1.03 \times 10^{4}$ \\
\hline $5 p^{5} 5 d$ & ${ }^{3} P^{o}$ & 2 & $5 p^{5} 5 g$ & ${ }^{3} F^{o}$ & 4 & 592.25 & -4.674 & $4.03 \times 10^{5}$ \\
\hline $5 p^{5} 5 d$ & ${ }^{3} F^{o}$ & 4 & $5 p^{5} 5 g$ & ${ }^{3} F^{o}$ & 4 & 595.33 & -6.069 & $1.61 \times 10^{4}$ \\
\hline $5 p^{5} 5 d$ & ${ }^{3} F^{o}$ & 3 & $5 p^{5} 5 g$ & ${ }^{3} F^{o}$ & 4 & 599.76 & -5.723 & $3.51 \times 10^{4}$ \\
\hline $5 p^{5} 5 d$ & ${ }^{3} F^{o}$ & 2 & $5 p^{5} 5 g$ & ${ }^{3} F^{o}$ & 4 & 611.66 & -5.602 & $4.46 \times 10^{4}$ \\
\hline $5 p^{5} 5 d$ & ${ }^{3} D^{o}$ & 3 & $5 p^{5} 5 g$ & ${ }^{3} F^{o}$ & 4 & 631.77 & -4.946 & $1.89 \times 10^{5}$ \\
\hline $5 p^{5} 5 d$ & ${ }^{3} D^{o}$ & 2 & $5 p^{5} 5 g$ & ${ }^{3} F^{o}$ & 4 & 697.81 & -5.706 & $2.69 \times 10^{4}$ \\
\hline $5 p^{5} 6 d$ & ${ }^{3} P^{o}$ & 2 & $5 p^{5} 5 g$ & ${ }^{3} F^{o}$ & 4 & 2195.46 & -4.544 & $3.95 \times 10^{4}$ \\
\hline $5 p^{5} 6 d$ & ${ }^{3} D^{o}$ & 3 & $5 p^{5} 5 g$ & ${ }^{3} F^{o}$ & 4 & 2280.11 & -4.943 & $1.46 \times 10^{4}$ \\
\hline $5 p^{5} 5 d$ & ${ }^{3} P^{o}$ & 1 & $5 p^{5} 5 g$ & ${ }^{3} G^{o}$ & 3 & 576.51 & -5.607 & $4.96 \times 10^{4}$ \\
\hline $5 p^{5} 5 d$ & ${ }^{3} F^{o}$ & 2 & $5 p^{5} 5 g$ & ${ }^{3} G^{o}$ & 3 & 611.14 & -5.265 & $9.69 \times 10^{4}$ \\
\hline $5 p^{5} 5 d$ & ${ }^{3} D^{o}$ & 3 & $5 p^{5} 5 g$ & ${ }^{3} G^{o}$ & 3 & 631.22 & -6.078 & $1.40 \times 10^{4}$ \\
\hline $5 p^{5} 5 d$ & ${ }^{3} D^{o}$ & 1 & $5 p^{5} 5 g$ & ${ }^{3} G^{o}$ & 3 & 662.32 & -5.151 & $1.08 \times 10^{5}$ \\
\hline $5 p^{5} 5 d$ & ${ }^{1} P^{o}$ & 1 & $5 p^{5} 5 g$ & ${ }^{3} G^{o}$ & 3 & 862.88 & -5.293 & $4.56 \times 10^{4}$ \\
\hline $5 p^{5} 6 d$ & ${ }^{1} P^{o}$ & 1 & $5 p^{5} 5 g$ & ${ }^{3} G^{o}$ & 3 & 2433.71 & -4.946 & $1.28 \times 10^{4}$ \\
\hline $5 p^{5} 5 d$ & ${ }^{3} P^{o}$ & 2 & $5 p^{5} 5 g$ & ${ }^{1} G^{o}$ & 4 & 591.37 & -6.192 & $1.23 \times 10^{4}$ \\
\hline $5 p^{5} 5 d$ & ${ }^{3} F^{o}$ & 4 & $5 p^{5} 5 g$ & ${ }^{1} G^{o}$ & 4 & 594.44 & -6.166 & $1.29 \times 10^{4}$ \\
\hline $5 p^{5} 5 d$ & ${ }^{3} F^{o}$ & 3 & $5 p^{5} 5 g$ & ${ }^{1} G^{o}$ & 4 & 598.86 & -4.995 & $1.88 \times 10^{5}$ \\
\hline $5 p^{5} 5 d$ & ${ }^{3} F^{o}$ & 2 & $5 p^{5} 5 g$ & ${ }^{1} G^{o}$ & 4 & 610.72 & -4.543 & $5.12 \times 10^{5}$ \\
\hline $5 p^{5} 5 d$ & ${ }^{3} F^{o}$ & 2 & $5 p^{5} 5 g$ & ${ }^{1} G^{o}$ & 4 & 680.61 & -5.874 & $1.92 \times 10^{4}$ \\
\hline $5 p^{5} 6 d$ & ${ }^{3} F^{o}$ & 3 & $5 p^{5} 5 g$ & ${ }^{1} G^{o}$ & 4 & 2182.56 & -4.96 & $1.53 \times 10^{4}$ \\
\hline $5 p^{5} 6 d$ & ${ }^{1} D^{o}$ & 2 & $5 p^{5} 5 g$ & ${ }^{1} G^{o}$ & 4 & 2242.8 & -4.491 & $4.28 \times 10^{4}$ \\
\hline $5 p^{5} 5 d$ & ${ }^{3} F^{o}$ & 4 & $5 p^{5} 5 g$ & ${ }^{3} G^{\circ}$ & 5 & 594.39 & -5.028 & $1.77 \times 10^{5}$ \\
\hline $5 p^{5} 5 d$ & ${ }^{3} F^{o}$ & 3 & $5 p^{5} 5 g$ & ${ }^{3} G^{\circ}$ & 5 & 598.81 & -5.352 & $8.27 \times 10^{4}$ \\
\hline $5 p^{5} 5 d$ & ${ }^{3} D^{o}$ & 3 & $5 p^{5} 5 g$ & ${ }^{3} G^{o}$ & 5 & 630.71 & -4.451 & $5.93 \times 10^{5}$ \\
\hline $5 p^{5} 6 d$ & ${ }^{3} F^{o}$ & 4 & $5 p^{5} 5 g$ & ${ }^{3} G^{o}$ & 5 & 2167.22 & -4.952 & $1.59 \times 10^{4}$ \\
\hline $5 p^{5} 6 d$ & ${ }^{3} D^{o}$ & 3 & $5 p^{5} 5 g$ & ${ }^{3} G^{\circ}$ & 5 & 2266.37 & -4.374 & $5.49 \times 10^{4}$ \\
\hline $5 p^{5} 6 s$ & ${ }^{1} P^{o}$ & 1 & $5 p^{5} 7 d$ & ${ }^{1} P^{o}$ & 1 & 748.98 & -5.756 & $2.09 \times 10^{4}$ \\
\hline $5 p^{5} 5 d$ & ${ }^{3} P^{o}$ & 1 & $5 p^{5} 7 d$ & ${ }^{3} F^{o}$ & 2 & 517.11 & -6.256 & $1.38 \times 10^{4}$ \\
\hline $5 p^{5} 5 d$ & ${ }^{3} F^{o}$ & 2 & $5 p^{5} 7 d$ & ${ }^{3} F^{o}$ & 2 & 599.74 & -6.041 & $1.69 \times 10^{4}$ \\
\hline $5 p^{5} 6 s$ & ${ }^{3} P^{o}$ & 1 & $5 p^{5} 7 d$ & ${ }^{3} F^{o}$ & 2 & 762.05 & -5.956 & $1.27 \times 10^{4}$ \\
\hline $5 p^{5} 5 d$ & ${ }^{3} D^{o}$ & 2 & $5 p^{5} 7 d$ & ${ }^{3} P^{o}$ & 2 & 611.22 & -6.011 & $1.74 \times 10^{4}$ \\
\hline $5 p^{5} 6 s$ & ${ }^{3} P^{o}$ & 0 & $5 p^{5} 7 d$ & ${ }^{3} P^{o}$ & 2 & 753.7 & -5.913 & $1.43 \times 10^{4}$ \\
\hline $5 p^{5} 5 d$ & ${ }^{3} P^{o}$ & 1 & $5 p^{5} 7 d$ & ${ }^{3} F^{o}$ & 3 & 516.01 & -6.197 & $1.59 \times 10^{4}$ \\
\hline $5 p^{5} 5 d$ & ${ }^{3} P^{o}$ & 2 & $5 p^{5} 7 d$ & ${ }^{3} F^{o}$ & 3 & 528.2 & -6.16 & $1.65 \times 10^{4}$ \\
\hline $5 p^{5} 5 d$ & ${ }^{1} F^{o}$ & 3 & $5 p^{5} 7 d$ & ${ }^{3} F^{o}$ & 3 & 619.76 & -5.797 & $2.77 \times 10^{4}$ \\
\hline $5 p^{5} 5 d$ & ${ }^{1} P^{o}$ & 1 & $5 p^{5} 7 d$ & ${ }^{3} F^{o}$ & 3 & 734.06 & -6.074 & $1.04 \times 10^{4}$ \\
\hline $5 p^{5} 6 s$ & ${ }^{3} P^{o}$ & 1 & $5 p^{5} 7 d$ & ${ }^{3} F^{o}$ & 3 & 759.66 & -5.555 & $3.22 \times 10^{4}$ \\
\hline
\end{tabular}


TABLE VI (cont.)

\begin{tabular}{|c|c|c|c|c|c|c|c|c|}
\hline \multicolumn{3}{|c|}{ Lower Level } & \multicolumn{3}{|c|}{ Upper Level } & $\lambda[\AA]$ & $\overline{\log (g f)}$ & $g A_{j i}\left[\mathrm{~s}^{-1}\right]$ \\
\hline Conf. & Term & $J$ & Conf. & Term & $J$ & HFR & HFR & HFR \\
\hline $5 p^{5} 7 s$ & ${ }^{3} P^{o}$ & 1 & $5 p^{5} 7 d$ & ${ }^{3} F^{o}$ & 3 & 2613.29 & -4.857 & $1.36 \times 10^{4}$ \\
\hline $5 p^{5} 5 d$ & ${ }^{3} P^{o}$ & 2 & $5 p^{5} 5 g$ & ${ }^{3} F^{o}$ & 4 & 527.05 & -5.044 & $2.17 \times 10^{5}$ \\
\hline $5 p^{5} 5 d$ & ${ }^{3} D^{o}$ & 3 & $5 p^{5} 5 g$ & ${ }^{3} F^{o}$ & 4 & 558.12 & -6.088 & $1.75 \times 10^{4}$ \\
\hline $5 p^{5} 5 d$ & ${ }^{3} D^{o}$ & 2 & $5 p^{5} 5 g$ & ${ }^{3} F^{o}$ & 4 & 609.04 & -4.609 & $4.42 \times 10^{5}$ \\
\hline $5 p^{5} 5 d$ & ${ }^{1} F^{o}$ & 3 & $5 p^{5} 5 g$ & ${ }^{3} F^{o}$ & 4 & 618.19 & -5.452 & $6.17 \times 10^{4}$ \\
\hline $5 p^{5} 6 d$ & ${ }^{3} P^{o}$ & 2 & $5 p^{5} 5 g$ & ${ }^{3} F^{o}$ & 4 & 2227.79 & -4.457 & $4.69 \times 10^{4}$ \\
\hline $5 p^{5} 5 d$ & ${ }^{3} F^{o}$ & 3 & $5 p^{5} 5 g$ & ${ }^{3} H^{o}$ & 4 & 532.83 & -6.047 & $2.11 \times 10^{4}$ \\
\hline $5 p^{5} 5 d$ & ${ }^{3} F^{o}$ & 2 & $5 p^{5} 5 g$ & ${ }^{3} H^{o}$ & 4 & 542.2 & -5.225 & $1.35 \times 10^{5}$ \\
\hline $5 p^{5} 5 d$ & ${ }^{3} F^{o}$ & 2 & $5 p^{5} 5 g$ & ${ }^{3} H^{o}$ & 4 & 596.59 & -4.486 & $6.11 \times 10^{5}$ \\
\hline $5 p^{5} 5 d$ & ${ }^{1} F^{o}$ & 3 & $5 p^{5} 5 g$ & ${ }^{3} H^{o}$ & 4 & 617.97 & -5.617 & $4.22 \times 10^{4}$ \\
\hline $5 p^{5} 6 d$ & ${ }^{3} F^{o}$ & 2 & $5 p^{5} 5 g$ & ${ }^{3} H^{o}$ & 4 & 2202.78 & -4.408 & $5.38 \times 10^{4}$ \\
\hline $5 p^{5} 5 d$ & ${ }^{3} P^{o}$ & 1 & $5 p^{5} 5 g$ & ${ }^{3} G^{o}$ & 3 & 514.75 & -5.487 & $8.20 \times 10^{4}$ \\
\hline $5 p^{5} 5 d$ & ${ }^{3} P^{o}$ & 2 & $5 p^{5} 5 g$ & ${ }^{3} G^{o}$ & 3 & 526.88 & -5.759 & $4.18 \times 10^{4}$ \\
\hline $5 p^{5} 5 d$ & ${ }^{3} D^{o}$ & 1 & $5 p^{5} 5 g$ & ${ }^{3} G^{o}$ & 3 & 582.09 & -5.285 & $1.02 \times 10^{5}$ \\
\hline $5 p^{5} 5 d$ & ${ }^{3} F^{o}$ & 2 & $5 p^{5} 5 g$ & ${ }^{3} G^{o}$ & 3 & 596.58 & -5.484 & $6.15 \times 10^{4}$ \\
\hline $5 p^{5} 5 d$ & ${ }^{3} D^{o}$ & 2 & $5 p^{5} 5 g$ & ${ }^{3} G^{o}$ & 3 & 608.82 & -5.741 & $3.27 \times 10^{4}$ \\
\hline $5 p^{5} 5 d$ & ${ }^{1} P^{o}$ & 1 & $5 p^{5} 5 g$ & ${ }^{3} G^{o}$ & 3 & 731.52 & -4.767 & $2.13 \times 10^{5}$ \\
\hline $5 p^{5} 6 d$ & ${ }^{3} D^{o}$ & 1 & $5 p^{5} 5 g$ & ${ }^{3} G^{o}$ & 3 & 2338.4 & -4.709 & $2.38 \times 10^{4}$ \\
\hline $5 p^{5} 5 d$ & ${ }^{3} F^{o}$ & 4 & $5 p^{5} 5 g$ & ${ }^{3} H^{o}$ & 5 & 529.24 & -6.345 & $1.08 \times 10^{4}$ \\
\hline $5 p^{5} 5 d$ & ${ }^{3} F^{o}$ & 3 & $5 p^{5} 5 g$ & ${ }^{3} H^{o}$ & 5 & 532.74 & -5.39 & $9.58 \times 10^{4}$ \\
\hline $5 p^{5} 5 d$ & ${ }^{3} D^{o}$ & 3 & $5 p^{5} 5 g$ & ${ }^{3} H^{o}$ & 5 & 557.84 & -6.172 & $1.44 \times 10^{4}$ \\
\hline $5 p^{5} 5 d$ & ${ }^{1} F^{o}$ & 3 & $5 p^{5} 5 g$ & ${ }^{3} H^{o}$ & 5 & 617.84 & -4.347 & $7.85 \times 10^{5}$ \\
\hline $5 p^{5} 6 d$ & ${ }^{3} F^{o}$ & 3 & $5 p^{5} 5 g$ & ${ }^{3} H^{o}$ & 5 & 2237.35 & -4.293 & $6.78 \times 10^{4}$ \\
\hline $5 p^{5} 5 d$ & ${ }^{3} P^{o}$ & 0 & $5 p^{5} 6 g$ & ${ }^{3} F^{o}$ & 2 & 507.52 & -5.296 & $1.31 \times 10^{5}$ \\
\hline $5 p^{5} 5 d$ & ${ }^{3} P^{o}$ & 1 & $5 p^{5} 6 g$ & ${ }^{3} F^{o}$ & 2 & 513.47 & -5.205 & $1.58 \times 10^{5}$ \\
\hline $5 p^{5} 5 d$ & ${ }^{3} P^{o}$ & 2 & $5 p^{5} 6 g$ & ${ }^{3} F^{o}$ & 2 & 525.54 & -5.823 & $3.63 \times 10^{4}$ \\
\hline $5 p^{5} 5 d$ & ${ }^{3} D^{o}$ & 1 & $5 p^{5} 6 g$ & ${ }^{3} F^{o}$ & 2 & 580.45 & -6.225 & $1.18 \times 10^{4}$ \\
\hline $5 p^{5} 5 d$ & ${ }^{3} P^{o}$ & 1 & $5 p^{5} 6 g$ & ${ }^{3} F^{o}$ & 3 & 513.28 & -5.507 & $7.88 \times 10^{4}$ \\
\hline $5 p^{5} 5 d$ & ${ }^{3} P^{o}$ & 2 & $5 p^{5} 6 g$ & ${ }^{3} F^{o}$ & 3 & 525.34 & -5.466 & $8.26 \times 10^{4}$ \\
\hline $5 p^{5} 5 d$ & ${ }^{3} D^{o}$ & 3 & $5 p^{5} 6 g$ & ${ }^{3} F^{o}$ & 3 & 556.2 & -5.971 & $2.30 \times 10^{4}$ \\
\hline $5 p^{5} 5 d$ & ${ }^{3} D^{o}$ & 1 & $5 p^{5} 6 g$ & ${ }^{3} F^{o}$ & 3 & 580.2 & -5.565 & $5.40 \times 10^{4}$ \\
\hline $5 p^{5} 5 d$ & ${ }^{3} D^{o}$ & 2 & $5 p^{5} 6 g$ & ${ }^{3} F^{o}$ & 3 & 606.75 & -6.202 & $1.14 \times 10^{4}$ \\
\hline $5 p^{5} 5 d$ & ${ }^{1} P^{o}$ & 1 & $5 p^{5} 6 g$ & ${ }^{3} F^{o}$ & 3 & 728.54 & -5.502 & $3.96 \times 10^{4}$ \\
\hline $5 p^{5} 5 d$ & ${ }^{3} F^{o}$ & 4 & $5 p^{5} 6 g$ & ${ }^{3} H^{o}$ & 6 & 527.75 & -4.421 & $9.08 \times 10^{5}$ \\
\hline $5 p^{5} 7 d$ & ${ }^{3} F^{o}$ & 4 & $5 p^{5} 6 g$ & ${ }^{3} H^{o}$ & 6 & 4450.61 & -4.334 & $1.56 \times 10^{4}$ \\
\hline $5 p^{5} 5 d$ & ${ }^{3} F^{o}$ & 4 & $5 p^{5} 6 g$ & ${ }^{1} H^{o}$ & 5 & 527.67 & -5.836 & $3.49 \times 10^{4}$ \\
\hline $5 p^{5} 5 d$ & ${ }^{3} F^{o}$ & 3 & $5 p^{5} 6 g$ & ${ }^{1} H^{o}$ & 5 & 531.15 & -4.638 & $5.44 \times 10^{5}$ \\
\hline $5 p^{5} 5 d$ & ${ }^{3} D^{o}$ & 3 & $5 p^{5} 6 g$ & ${ }^{1} H^{o}$ & 5 & 556.1 & -5.211 & $1.33 \times 10^{5}$ \\
\hline $5 p^{5} 5 d$ & ${ }^{1} F^{o}$ & 3 & $5 p^{5} 6 g$ & ${ }^{1} H^{o}$ & 5 & 615.7 & -5.736 & $3.23 \times 10^{4}$ \\
\hline $5 p^{5} 7 d$ & ${ }^{3} F^{o}$ & 3 & $5 p^{5} 6 g$ & ${ }^{1} H^{o}$ & 5 & 4449.72 & -4.463 & $1.16 \times 10^{4}$ \\
\hline $5 p^{5} 5 d$ & ${ }^{3} F^{o}$ & 4 & $5 p^{5} 8 d$ & ${ }^{3} F^{o}$ & 4 & 527.35 & -5.991 & $2.45 \times 10^{4}$ \\
\hline $5 p^{5} 5 d$ & ${ }^{3} D^{o}$ & 2 & $5 p^{5} 8 d$ & ${ }^{3} F^{o}$ & 4 & 606.22 & -5.991 & $1.85 \times 10^{4}$ \\
\hline $5 p^{5} 6 s$ & ${ }^{3} P^{o}$ & 2 & $5 p^{5} 8 d$ & ${ }^{3} F^{o}$ & 4 & 645.06 & -5.86 & $2.21 \times 10^{4}$ \\
\hline $5 p^{5} 5 d$ & ${ }^{3} P^{o}$ & 1 & $5 p^{5} 8 d$ & ${ }^{3} F^{o}$ & 3 & 512.53 & -5.739 & $4.63 \times 10^{4}$ \\
\hline $5 p^{5} 5 d$ & ${ }^{3} P^{o}$ & 2 & $5 p^{5} 8 d$ & ${ }^{3} F^{o}$ & 3 & 524.56 & -5.951 & $2.72 \times 10^{4}$ \\
\hline $5 p^{5} 5 d$ & ${ }^{3} F^{o}$ & 3 & $5 p^{5} 8 d$ & ${ }^{3} F^{o}$ & 3 & 530.44 & -6.009 & $2.32 \times 10^{4}$ \\
\hline $5 p^{5} 5 d$ & ${ }^{3} D^{o}$ & 1 & $5 p^{5} 8 d$ & ${ }^{3} F^{o}$ & 3 & 579.25 & -5.83 & $2.94 \times 10^{4}$ \\
\hline $5 p^{5} 5 d$ & ${ }^{3} F^{o}$ & 2 & $5 p^{5} 8 d$ & ${ }^{3} F^{o}$ & 3 & 593.59 & -6.247 & $1.07 \times 10^{4}$ \\
\hline $5 p^{5} 5 d$ & ${ }^{3} D^{o}$ & 2 & $5 p^{5} 8 d$ & ${ }^{3} F^{o}$ & 3 & 605.71 & -5.935 & $2.11 \times 10^{4}$ \\
\hline $5 p^{5} 5 d$ & ${ }^{1} P^{o}$ & 1 & $5 p^{5} 8 d$ & ${ }^{3} F^{o}$ & 3 & 727.04 & -5.635 & $2.92 \times 10^{4}$ \\
\hline $5 p^{5} 6 s$ & ${ }^{3} P^{o}$ & 2 & $5 p^{5} 8 d$ & ${ }^{3} D^{o}$ & 2 & 643.91 & -6.156 & $1.12 \times 10^{4}$ \\
\hline $5 p^{5} 5 d$ & ${ }^{3} P^{o}$ & 2 & $5 p^{5} 6 g$ & ${ }^{3} F^{o}$ & 4 & 524.13 & -5.093 & $1.96 \times 10^{5}$ \\
\hline
\end{tabular}


TABLE VI (cont.)

\begin{tabular}{|c|c|c|c|c|c|c|c|c|}
\hline \multicolumn{3}{|c|}{ Lower Level } & \multicolumn{3}{|c|}{ Upper Level } & $\lambda[\AA]$ & $\log (g f)$ & $g A_{j i}\left[\mathrm{~s}^{-1}\right]$ \\
\hline Conf. & Term & $J$ & Conf. & Term & $J$ & HFR & HFR & HFR \\
\hline $5 p^{5} 5 d$ & ${ }^{3} F^{o}$ & 4 & $5 p^{5} 6 g$ & ${ }^{3} F^{o}$ & 4 & 526.55 & -6.316 & $1.16 \times 10^{4}$ \\
\hline $5 p^{5} 5 d$ & ${ }^{3} F^{o}$ & 3 & $5 p^{5} 6 g$ & ${ }^{3} F^{o}$ & 4 & 530.01 & -5.886 & $3.09 \times 10^{4}$ \\
\hline $5 p^{5} 5 d$ & ${ }^{3} F^{o}$ & 2 & $5 p^{5} 6 g$ & ${ }^{3} F^{o}$ & 4 & 539.27 & -5.903 & $2.87 \times 10^{4}$ \\
\hline $5 p^{5} 5 d$ & ${ }^{3} D^{o}$ & 3 & $5 p^{5} 6 g$ & ${ }^{3} F^{o}$ & 4 & 554.85 & -5.218 & $1.31 \times 10^{5}$ \\
\hline $5 p^{5} 5 d$ & ${ }^{3} D^{o}$ & 2 & $5 p^{5} 6 g$ & ${ }^{3} F^{o}$ & 4 & 605.15 & -5.025 & $1.72 \times 10^{5}$ \\
\hline $5 p^{5} 5 d$ & ${ }^{3} P^{o}$ & 1 & $5 p^{5} 6 g$ & ${ }^{3} G^{o}$ & 3 & 512.03 & -6.035 & $2.35 \times 10^{4}$ \\
\hline $5 p^{5} 5 d$ & ${ }^{3} P^{o}$ & 2 & $5 p^{5} 6 g$ & ${ }^{3} G^{o}$ & 3 & 524.03 & -5.936 & $2.82 \times 10^{4}$ \\
\hline $5 p^{5} 5 d$ & ${ }^{3} F^{o}$ & 3 & $5 p^{5} 6 g$ & ${ }^{3} G^{o}$ & 3 & 529.9 & -6.258 & $1.31 \times 10^{4}$ \\
\hline $5 p^{5} 5 d$ & ${ }^{3} F^{o}$ & 2 & $5 p^{5} 6 g$ & ${ }^{3} G^{o}$ & 3 & 539.16 & -5.294 & $1.17 \times 10^{5}$ \\
\hline $5 p^{5} 5 d$ & ${ }^{3} D^{o}$ & 1 & $5 p^{5} 6 g$ & ${ }^{3} G^{o}$ & 3 & 578.6 & -5.054 & $1.76 \times 10^{5}$ \\
\hline $5 p^{5} 5 d$ & ${ }^{3} F^{o}$ & 4 & $5 p^{5} 6 g$ & ${ }^{1} G^{o}$ & 4 & 526.34 & -6.379 & $1.01 \times 10^{4}$ \\
\hline $5 p^{5} 5 d$ & ${ }^{3} F^{o}$ & 3 & $5 p^{5} 6 g$ & ${ }^{1} G^{o}$ & 4 & 529.8 & -5.249 & $1.34 \times 10^{5}$ \\
\hline $5 p^{5} 5 d$ & ${ }^{3} F^{o}$ & 2 & $5 p^{5} 6 g$ & ${ }^{1} G^{o}$ & 4 & 539.06 & -4.853 & $3.22 \times 10^{5}$ \\
\hline $5 p^{5} 5 d$ & ${ }^{3} F^{o}$ & 2 & $5 p^{5} 6 g$ & ${ }^{1} G^{o}$ & 4 & 592.79 & -5.232 & $1.11 \times 10^{5}$ \\
\hline $5 p^{5} 5 d$ & ${ }^{1} F^{o}$ & 3 & $5 p^{5} 6 g$ & ${ }^{1} G^{o}$ & 4 & 613.89 & -6.088 & $1.44 \times 10^{4}$ \\
\hline $5 p^{5} 5 d$ & ${ }^{3} F^{o}$ & 4 & $5 p^{5} 6 g$ & ${ }^{3} G^{o}$ & 5 & 526.33 & -5.238 & $1.39 \times 10^{5}$ \\
\hline $5 p^{5} 5 d$ & ${ }^{3} F^{o}$ & 3 & $5 p^{5} 6 g$ & ${ }^{3} G^{o}$ & 5 & 529.79 & -5.724 & $4.48 \times 10^{4}$ \\
\hline $5 p^{5} 5 d$ & ${ }^{3} D^{o}$ & 3 & $5 p^{5} 6 g$ & ${ }^{3} G^{o}$ & 5 & 554.61 & -4.682 & $4.51 \times 10^{5}$ \\
\hline $5 p^{5} 5 d$ & ${ }^{1} F^{o}$ & 3 & $5 p^{5} 6 g$ & ${ }^{3} G^{o}$ & 5 & 613.88 & -5.463 & $6.10 \times 10^{4}$ \\
\hline $5 p^{5} 6 s$ & ${ }^{1} P^{o}$ & 1 & $5 p^{5} 8 d$ & ${ }^{1} D^{o}$ & 2 & 651.08 & -6.166 & $1.07 \times 10^{4}$ \\
\hline $5 p^{5} 5 d$ & ${ }^{3} P^{o}$ & 0 & $5 p^{5} 7 g$ & ${ }^{3} F^{o}$ & 2 & 475.54 & -5.442 & $1.07 \times 10^{5}$ \\
\hline $5 p^{5} 5 d$ & ${ }^{3} P^{o}$ & 1 & $5 p^{5} 7 g$ & ${ }^{3} F^{o}$ & 2 & 480.77 & -5.366 & $1.24 \times 10^{5}$ \\
\hline $5 p^{5} 5 d$ & ${ }^{3} P^{o}$ & 2 & $5 p^{5} 7 g$ & ${ }^{3} F^{o}$ & 2 & 491.33 & -5.991 & $2.82 \times 10^{4}$ \\
\hline $5 p^{5} 5 d$ & ${ }^{3} P^{o}$ & 1 & $5 p^{5} 7 g$ & ${ }^{3} F^{o}$ & 3 & 480.67 & -5.369 & $1.23 \times 10^{5}$ \\
\hline $5 p^{5} 5 d$ & ${ }^{3} P^{o}$ & 2 & $5 p^{5} 7 g$ & ${ }^{3} F^{o}$ & 3 & 491.22 & -5.431 & $1.02 \times 10^{5}$ \\
\hline $5 p^{5} 5 d$ & ${ }^{3} D^{o}$ & 3 & $5 p^{5} 7 g$ & ${ }^{3} F^{o}$ & 3 & 518.1 & -6.186 & $1.62 \times 10^{4}$ \\
\hline $5 p^{5} 5 d$ & ${ }^{3} D^{o}$ & 1 & $5 p^{5} 7 g$ & ${ }^{3} F^{o}$ & 3 & 538.87 & -5.906 & $2.85 \times 10^{4}$ \\
\hline $5 p^{5} 5 d$ & ${ }^{1} P^{o}$ & 1 & $5 p^{5} 7 g$ & ${ }^{3} F^{o}$ & 3 & 664.55 & -5.969 & $1.62 \times 10^{4}$ \\
\hline $5 p^{5} 5 d$ & ${ }^{3} F^{o}$ & 4 & $5 p^{5} 7 g$ & ${ }^{3} H^{o}$ & 6 & 493.23 & -4.608 & $6.76 \times 10^{5}$ \\
\hline $5 p^{5} 6 d$ & ${ }^{3} F^{o}$ & 4 & $5 p^{5} 7 g$ & ${ }^{3} H^{o}$ & 6 & 1239.98 & -5.572 & $1.16 \times 10^{4}$ \\
\hline $5 p^{5} 5 d$ & ${ }^{3} F^{o}$ & 4 & $5 p^{5} 7 g$ & ${ }^{1} H^{o}$ & 5 & 493.17 & -5.937 & $3.17 \times 10^{4}$ \\
\hline $5 p^{5} 5 d$ & ${ }^{3} F^{o}$ & 3 & $5 p^{5} 7 g$ & ${ }^{1} H^{o}$ & 5 & 496.21 & -4.811 & $4.19 \times 10^{5}$ \\
\hline $5 p^{5} 5 d$ & ${ }^{3} D^{o}$ & 3 & $5 p^{5} 7 g$ & ${ }^{1} H^{o}$ & 5 & 517.92 & -5.527 & $7.39 \times 10^{4}$ \\
\hline $5 p^{5} 5 d$ & ${ }^{3} P^{o}$ & 2 & $5 p^{5} 7 g$ & ${ }^{3} F^{o}$ & 4 & 490.76 & -5.189 & $1.79 \times 10^{5}$ \\
\hline $5 p^{5} 5 d$ & ${ }^{3} F^{o}$ & 4 & $5 p^{5} 7 g$ & ${ }^{3} F^{o}$ & 4 & 492.87 & -6.024 & $2.60 \times 10^{4}$ \\
\hline $5 p^{5} 5 d$ & ${ }^{3} F^{o}$ & 2 & $5 p^{5} 7 g$ & ${ }^{3} F^{o}$ & 4 & 504.01 & -6.229 & $1.55 \times 10^{4}$ \\
\hline $5 p^{5} 5 d$ & ${ }^{3} D^{o}$ & 3 & $5 p^{5} 7 g$ & ${ }^{3} F^{o}$ & 4 & 517.59 & -5.627 & $5.88 \times 10^{4}$ \\
\hline $5 p^{5} 5 d$ & ${ }^{3} D^{o}$ & 2 & $5 p^{5} 7 g$ & ${ }^{3} F^{o}$ & 4 & 561.09 & -6.208 & $1.31 \times 10^{4}$ \\
\hline $5 p^{5} 5 d$ & ${ }^{3} P^{o}$ & 1 & $5 p^{5} 7 g$ & ${ }^{3} G^{o}$ & 3 & 480.22 & -5.995 & $2.93 \times 10^{4}$ \\
\hline $5 p^{5} 5 d$ & ${ }^{3} P^{o}$ & 2 & $5 p^{5} 7 g$ & ${ }^{3} G^{o}$ & 3 & 490.76 & -6.272 & $1.48 \times 10^{4}$ \\
\hline $5 p^{5} 5 d$ & ${ }^{3} F^{o}$ & 3 & $5 p^{5} 7 g$ & ${ }^{3} G^{o}$ & 3 & 495.9 & -6.034 & $2.51 \times 10^{4}$ \\
\hline $5 p^{5} 5 d$ & ${ }^{3} F^{o}$ & 2 & $5 p^{5} 7 g$ & ${ }^{3} G^{o}$ & 3 & 504.01 & -5.987 & $2.71 \times 10^{4}$ \\
\hline $5 p^{5} 5 d$ & ${ }^{3} D^{o}$ & 1 & $5 p^{5} 7 g$ & ${ }^{3} G^{o}$ & 3 & 538.31 & -5.606 & $5.70 \times 10^{4}$ \\
\hline $5 p^{5} 5 d$ & ${ }^{1} P^{o}$ & 1 & $5 p^{5} 7 g$ & ${ }^{3} G^{o}$ & 3 & 663.69 & -6.005 & $1.50 \times 10^{4}$ \\
\hline $5 p^{5} 5 d$ & ${ }^{3} P^{o}$ & 2 & $5 p^{5} 9 d$ & ${ }^{3} F^{o}$ & 4 & 490.49 & -5.512 & $8.53 \times 10^{4}$ \\
\hline $5 p^{5} 5 d$ & ${ }^{3} F^{o}$ & 3 & $5 p^{5} 9 d$ & ${ }^{3} F^{o}$ & 4 & 495.63 & -6.272 & $1.45 \times 10^{4}$ \\
\hline $5 p^{5} 5 d$ & ${ }^{3} F^{o}$ & 2 & $5 p^{5} 9 d$ & ${ }^{3} F^{o}$ & 4 & 503.72 & -6.384 & $1.09 \times 10^{4}$ \\
\hline $5 p^{5} 5 d$ & ${ }^{3} D^{o}$ & 3 & $5 p^{5} 9 d$ & ${ }^{3} F^{o}$ & 4 & 517.29 & -5.722 & $4.73 \times 10^{4}$ \\
\hline $5 p^{5} 5 d$ & ${ }^{3} F^{o}$ & 3 & $5 p^{5} 7 g$ & ${ }^{1} G^{o}$ & 4 & 495.6 & -5.392 & $1.10 \times 10^{5}$ \\
\hline $5 p^{5} 5 d$ & ${ }^{3} F^{o}$ & 2 & $5 p^{5} 7 g$ & ${ }^{1} G^{o}$ & 4 & 503.69 & -4.937 & $3.04 \times 10^{5}$ \\
\hline $5 p^{5} 5 d$ & ${ }^{3} F^{o}$ & 4 & $5 p^{5} 7 g$ & ${ }^{3} G^{o}$ & 5 & 492.55 & -5.419 & $1.05 \times 10^{5}$ \\
\hline $5 p^{5} 5 d$ & ${ }^{3} F^{o}$ & 3 & $5 p^{5} 7 g$ & ${ }^{3} G^{o}$ & 5 & 495.58 & -5.769 & $4.63 \times 10^{4}$ \\
\hline
\end{tabular}


TABLE VI (cont.)

\begin{tabular}{|c|c|c|c|c|c|c|c|c|}
\hline \multicolumn{3}{|c|}{ Lower Level } & \multicolumn{3}{|c|}{ Upper Level } & $\lambda[\AA]$ & $\log (g f)$ & $g A_{j i}\left[\mathrm{~s}^{-1}\right]$ \\
\hline Conf. & Term & $J$ & Conf. & Term & $J$ & HFR & HFR & HFR \\
\hline $5 p^{5} 5 d$ & ${ }^{3} D^{o}$ & 3 & $5 p^{5} 7 g$ & ${ }^{3} G^{o}$ & 5 & 517.23 & -4.857 & $3.47 \times 10^{5}$ \\
\hline $5 p^{5} 5 d$ & ${ }^{3} P^{o}$ & 1 & $5 p^{5} 9 d$ & ${ }^{3} F^{o}$ & 3 & 479.86 & -6.21 & $1.79 \times 10^{4}$ \\
\hline $5 p^{5} 5 d$ & ${ }^{3} P^{o}$ & 2 & $5 p^{5} 9 d$ & ${ }^{3} F^{o}$ & 3 & 490.38 & -6.067 & $2.38 \times 10^{4}$ \\
\hline $5 p^{5} 5 d$ & ${ }^{3} F^{o}$ & 2 & $5 p^{5} 9 d$ & ${ }^{3} F^{o}$ & 3 & 503.61 & -5.711 & $5.12 \times 10^{4}$ \\
\hline $5 p^{5} 5 d$ & ${ }^{3} D^{o}$ & 1 & $5 p^{5} 9 d$ & ${ }^{3} F^{o}$ & 3 & 537.86 & -6.023 & $2.19 \times 10^{4}$ \\
\hline $5 p^{5} 5 d$ & ${ }^{3} P^{o}$ & 2 & $5 p^{5} 9 d$ & ${ }^{3} D^{o}$ & 3 & 489.78 & -6.351 & $1.24 \times 10^{4}$ \\
\hline $5 p^{5} 7 d$ & ${ }^{3} P^{o}$ & 2 & $5 p^{5} 7 i$ & ${ }^{3} H^{o}$ & 4 & 2769.47 & -4.855 & $1.21 \times 10^{4}$ \\
\hline $5 p^{5} 5 g$ & ${ }^{3} F^{o}$ & 2 & $5 p^{5} 7 i$ & ${ }^{3} H^{o}$ & 4 & 2771.93 & -4.695 & $1.75 \times 10^{4}$ \\
\hline $5 p^{5} 5 g$ & ${ }^{3} F^{o}$ & 3 & $5 p^{5} 7 i$ & ${ }^{3} H^{o}$ & 5 & 2777.44 & -4.377 & $3.63 \times 10^{4}$ \\
\hline $5 p^{5} 5 g$ & ${ }^{1} H^{o}$ & 5 & $5 p^{5} 7 i$ & ${ }^{1} K^{o}$ & 7 & 2791.56 & -4.145 & $6.12 \times 10^{4}$ \\
\hline $5 p^{5} 5 g$ & ${ }^{3} H^{o}$ & 6 & $5 p^{5} 7 i$ & ${ }^{3} K^{o}$ & 8 & 2788.73 & -4.082 & $7.10 \times 10^{4}$ \\
\hline $5 p^{5} 7 d$ & ${ }^{3} D^{o}$ & 3 & $5 p^{5} 7 i$ & ${ }^{3} I^{o}$ & 5 & 2821.03 & -4.755 & $1.47 \times 10^{4}$ \\
\hline $5 p^{5} 5 g$ & ${ }^{3} G^{o}$ & 3 & $5 p^{5} 7 i$ & ${ }^{3} I^{o}$ & 5 & 2834.59 & -4.598 & $2.10 \times 10^{4}$ \\
\hline $5 p^{5} 5 g$ & ${ }^{3} F^{o}$ & 4 & $5 p^{5} 7 i$ & ${ }^{3} H^{o}$ & 6 & 2823.52 & -4.267 & $4.52 \times 10^{4}$ \\
\hline $5 p^{5} 5 g$ & ${ }^{1} G^{o}$ & 4 & $5 p^{5} 7 i$ & ${ }^{1} I^{o}$ & 6 & 2842.19 & -4.248 & $4.66 \times 10^{4}$ \\
\hline $5 p^{5} 5 g$ & ${ }^{3} G^{o}$ & 5 & $5 p^{5} 7 i$ & ${ }^{3} I^{o}$ & 7 & 2843.29 & -4.172 & $5.56 \times 10^{4}$ \\
\hline $5 p^{5} 5 d$ & ${ }^{1} P^{o}$ & 1 & $5 p^{5} 10 s$ & ${ }^{1} P^{o}$ & 1 & 659.98 & -5.892 & $1.96 \times 10^{4}$ \\
\hline $5 p^{5} 5 d$ & ${ }^{3} F^{o}$ & 2 & $5 p^{5} 8 d$ & ${ }^{3} F^{o}$ & 2 & 527.78 & -6.34 & $1.09 \times 10^{4}$ \\
\hline $5 p^{5} 5 d$ & ${ }^{3} P^{o}$ & 2 & $5 p^{5} 6 g$ & ${ }^{3} F^{o}$ & 4 & 472.34 & -5.322 & $1.43 \times 10^{5}$ \\
\hline $5 p^{5} 5 d$ & ${ }^{3} D^{o}$ & 2 & $5 p^{5} 6 g$ & ${ }^{3} F^{o}$ & 4 & 537.14 & -4.735 & $4.26 \times 10^{5}$ \\
\hline $5 p^{5} 5 d$ & ${ }^{1} F^{o}$ & 3 & $5 p^{5} 6 g$ & ${ }^{3} F^{o}$ & 4 & 544.24 & -5.641 & $5.15 \times 10^{4}$ \\
\hline $5 p^{5} 5 d$ & ${ }^{3} F^{o}$ & 2 & $5 p^{5} 6 g$ & ${ }^{3} H^{o}$ & 4 & 484.56 & -5.555 & $7.92 \times 10^{4}$ \\
\hline $5 p^{5} 5 d$ & ${ }^{3} F^{o}$ & 2 & $5 p^{5} 6 g$ & ${ }^{3} H^{o}$ & 4 & 527.54 & -4.659 & $5.26 \times 10^{5}$ \\
\hline $5 p^{5} 5 d$ & ${ }^{1} F^{o}$ & 3 & $5 p^{5} 6 g$ & ${ }^{3} H^{o}$ & 4 & 544.19 & -5.764 & $3.88 \times 10^{4}$ \\
\hline $5 p^{5} 5 d$ & ${ }^{3} F^{o}$ & 3 & $5 p^{5} 6 g$ & ${ }^{3} H^{o}$ & 5 & 477.02 & -5.654 & $6.50 \times 10^{4}$ \\
\hline $5 p^{5} 5 d$ & ${ }^{1} F^{o}$ & 3 & $5 p^{5} 6 g$ & ${ }^{3} H^{o}$ & 5 & 544.13 & -4.539 & $6.51 \times 10^{5}$ \\
\hline $5 p^{5} 7 d$ & ${ }^{3} F^{o}$ & 3 & $5 p^{5} 6 g$ & ${ }^{3} H^{o}$ & 5 & 4459.01 & -4.485 & $1.10 \times 10^{4}$ \\
\hline $5 p^{5} 5 d$ & ${ }^{3} P^{o}$ & 1 & $5 p^{5} 6 g$ & ${ }^{3} G^{o}$ & 3 & 462.44 & -5.808 & $4.85 \times 10^{4}$ \\
\hline $5 p^{5} 5 d$ & ${ }^{3} P^{o}$ & 2 & $5 p^{5} 6 g$ & ${ }^{3} G^{o}$ & 3 & 472.21 & -6.278 & $1.58 \times 10^{4}$ \\
\hline $5 p^{5} 5 d$ & ${ }^{3} D^{o}$ & 1 & $5 p^{5} 6 g$ & ${ }^{3} G^{o}$ & 3 & 516.07 & -5.313 & $1.22 \times 10^{5}$ \\
\hline $5 p^{5} 5 d$ & ${ }^{3} F^{o}$ & 2 & $5 p^{5} 6 g$ & ${ }^{3} G^{o}$ & 3 & 527.43 & -5.731 & $4.45 \times 10^{4}$ \\
\hline $5 p^{5} 5 d$ & ${ }^{3} D^{o}$ & 2 & $5 p^{5} 6 g$ & ${ }^{3} G^{o}$ & 3 & 536.98 & -5.884 & $3.02 \times 10^{4}$ \\
\hline $5 p^{5} 5 d$ & ${ }^{1} F^{o}$ & 3 & $5 p^{5} 6 g$ & ${ }^{3} G^{o}$ & 3 & 544.07 & -6.293 & $1.15 \times 10^{4}$ \\
\hline $5 p^{5} 5 d$ & ${ }^{1} P^{o}$ & 1 & $5 p^{5} 6 g$ & ${ }^{3} G^{o}$ & 3 & 630.22 & -5.131 & $1.24 \times 10^{5}$ \\
\hline $5 p^{5} 5 d$ & ${ }^{3} D^{o}$ & 2 & $5 p^{5} 8 d$ & ${ }^{3} P^{o}$ & 2 & 536.94 & -6.16 & $1.60 \times 10^{4}$ \\
\hline $5 p^{5} 5 d$ & ${ }^{3} P^{o}$ & 1 & $5 p^{5} 8 d$ & ${ }^{3} F^{o}$ & 3 & 462.25 & -6.209 & $1.93 \times 10^{4}$ \\
\hline $5 p^{5} 5 d$ & ${ }^{3} P^{o}$ & 2 & $5 p^{5} 8 d$ & ${ }^{3} F^{o}$ & 3 & 472 & -5.911 & $3.67 \times 10^{4}$ \\
\hline $5 p^{5} 5 d$ & ${ }^{1} F^{o}$ & 3 & $5 p^{5} 8 d$ & ${ }^{3} F^{o}$ & 3 & 543.8 & -6.257 & $1.25 \times 10^{4}$ \\
\hline $5 p^{5} 6 s$ & ${ }^{3} P^{o}$ & 1 & $5 p^{5} 8 d$ & ${ }^{3} F^{o}$ & 3 & 648.61 & -6.192 & $1.02 \times 10^{4}$ \\
\hline $5 p^{5} 5 d$ & ${ }^{3} P^{o}$ & 0 & $5 p^{5} 8 g$ & ${ }^{3} F^{o}$ & 2 & 456.73 & -5.455 & $1.12 \times 10^{5}$ \\
\hline $5 p^{5} 5 d$ & ${ }^{3} P^{o}$ & 1 & $5 p^{5} 8 g$ & ${ }^{3} F^{o}$ & 2 & 461.55 & -5.414 & $1.21 \times 10^{5}$ \\
\hline $5 p^{5} 5 d$ & ${ }^{3} P^{o}$ & 2 & $5 p^{5} 8 g$ & ${ }^{3} F^{o}$ & 2 & 471.28 & -6.096 & $2.41 \times 10^{4}$ \\
\hline $5 p^{5} 5 d$ & ${ }^{3} F^{o}$ & 4 & $5 p^{5} 8 g$ & ${ }^{3} H^{o}$ & 6 & 473.16 & -4.667 & $6.41 \times 10^{5}$ \\
\hline $5 p^{5} 6 d$ & ${ }^{3} F^{o}$ & 4 & $5 p^{5} 8 g$ & ${ }^{3} H^{o}$ & 6 & 1120.48 & -5.448 & $1.89 \times 10^{4}$ \\
\hline $5 p^{5} 5 d$ & ${ }^{3} F^{o}$ & 4 & $5 p^{5} 8 g$ & ${ }^{1} H^{o}$ & 5 & 473.12 & -6.057 & $2.62 \times 10^{4}$ \\
\hline $5 p^{5} 5 d$ & ${ }^{3} F^{o}$ & 3 & $5 p^{5} 8 g$ & ${ }^{1} H^{o}$ & 5 & 475.92 & -4.975 & $3.12 \times 10^{5}$ \\
\hline $5 p^{5} 5 d$ & ${ }^{3} D^{o}$ & 3 & $5 p^{5} 8 g$ & ${ }^{1} H^{o}$ & 5 & 495.85 & -5.79 & $4.40 \times 10^{4}$ \\
\hline $5 p^{5} 6 d$ & ${ }^{3} F^{o}$ & 3 & $5 p^{5} 8 g$ & ${ }^{1} H^{o}$ & 5 & 1124.17 & -5.617 & $1.27 \times 10^{4}$ \\
\hline $5 p^{5} 5 d$ & ${ }^{3} P^{o}$ & 1 & $5 p^{5} 8 g$ & ${ }^{3} F^{o}$ & 3 & 461.39 & -5.643 & $7.12 \times 10^{4}$ \\
\hline $5 p^{5} 5 d$ & ${ }^{3} P^{o}$ & 2 & $5 p^{5} 8 g$ & ${ }^{3} F^{o}$ & 3 & 471.12 & -5.776 & $5.03 \times 10^{4}$ \\
\hline $5 p^{5} 5 d$ & ${ }^{3} D^{o}$ & 3 & $5 p^{5} 8 g$ & ${ }^{3} F^{o}$ & 3 & 495.78 & -6.261 & $1.49 \times 10^{4}$ \\
\hline $5 p^{5} 5 d$ & ${ }^{3} D^{o}$ & 2 & $5 p^{5} 8 g$ & ${ }^{3} F^{o}$ & 3 & 535.56 & -6.319 & $1.12 \times 10^{4}$ \\
\hline
\end{tabular}


TABLE VI (cont.)

\begin{tabular}{|c|c|c|c|c|c|c|c|c|}
\hline \multicolumn{3}{|c|}{ Lower Level } & \multicolumn{3}{|c|}{ Upper Level } & $\lambda[\AA]$ & $\log (g f)$ & $g A_{j i}\left[\mathrm{~s}^{-1}\right]$ \\
\hline Conf. & Term & $J$ & Conf. & Term & $J$ & HFR & HFR & HFR \\
\hline $5 p^{5} 6 s$ & ${ }^{3} P^{o}$ & 1 & $5 p^{5} 8 g$ & ${ }^{3} F^{o}$ & 3 & 646.93 & -6.093 & $1.29 \times 10^{4}$ \\
\hline $5 p^{5} 5 d$ & ${ }^{3} P^{o}$ & 2 & $5 p^{5} 8 g$ & ${ }^{3} F^{o}$ & 4 & 470.83 & -5.172 & $2.02 \times 10^{5}$ \\
\hline $5 p^{5} 5 d$ & ${ }^{3} F^{o}$ & 3 & $5 p^{5} 8 g$ & ${ }^{3} F^{o}$ & 4 & 475.57 & -6.368 & $1.27 \times 10^{4}$ \\
\hline $5 p^{5} 5 d$ & ${ }^{3} F^{o}$ & 2 & $5 p^{5} 8 g$ & ${ }^{3} F^{o}$ & 4 & 483.02 & -6.271 & $1.53 \times 10^{4}$ \\
\hline $5 p^{5} 5 d$ & ${ }^{3} D^{o}$ & 3 & $5 p^{5} 8 g$ & ${ }^{3} F^{o}$ & 4 & 495.47 & -5.875 & $3.62 \times 10^{4}$ \\
\hline $5 p^{5} 5 d$ & ${ }^{3} D^{o}$ & 2 & $5 p^{5} 8 g$ & ${ }^{3} F^{o}$ & 4 & 535.2 & -5.618 & $5.61 \times 10^{4}$ \\
\hline $5 p^{5} 5 d$ & ${ }^{3} P^{o}$ & 1 & $5 p^{5} 8 g$ & ${ }^{3} G^{o}$ & 3 & 461.12 & -5.739 & $5.72 \times 10^{4}$ \\
\hline $5 p^{5} 5 d$ & ${ }^{3} P^{o}$ & 2 & $5 p^{5} 8 g$ & ${ }^{3} G^{o}$ & 3 & 470.83 & -5.883 & $3.94 \times 10^{4}$ \\
\hline $5 p^{5} 5 d$ & ${ }^{3} F^{o}$ & 3 & $5 p^{5} 8 g$ & ${ }^{3} G^{o}$ & 3 & 475.56 & -6.153 & $2.07 \times 10^{4}$ \\
\hline $5 p^{5} 5 d$ & ${ }^{3} F^{o}$ & 2 & $5 p^{5} 8 g$ & ${ }^{3} G^{o}$ & 3 & 483.01 & -5.913 & $3.50 \times 10^{4}$ \\
\hline $5 p^{5} 5 d$ & ${ }^{3} D^{o}$ & 1 & $5 p^{5} 8 g$ & ${ }^{3} G^{o}$ & 3 & 514.43 & -5.594 & $6.42 \times 10^{4}$ \\
\hline $5 p^{5} 5 d$ & ${ }^{1} P^{o}$ & 1 & $5 p^{5} 8 g$ & ${ }^{3} G^{o}$ & 3 & 627.76 & -5.838 & $2.46 \times 10^{4}$ \\
\hline $5 p^{5} 5 d$ & ${ }^{3} F^{o}$ & 3 & $5 p^{5} 8 g$ & ${ }^{1} G^{o}$ & 4 & 475.47 & -5.57 & $7.94 \times 10^{4}$ \\
\hline $5 p^{5} 5 d$ & ${ }^{3} F^{o}$ & 2 & $5 p^{5} 8 g$ & ${ }^{1} G^{o}$ & 4 & 482.91 & -5.179 & $1.90 \times 10^{5}$ \\
\hline $5 p^{5} 5 d$ & ${ }^{3} F^{o}$ & 2 & $5 p^{5} 8 g$ & ${ }^{1} G^{o}$ & 4 & 525.59 & -5.753 & $4.26 \times 10^{4}$ \\
\hline $5 p^{5} 5 d$ & ${ }^{3} F^{o}$ & 4 & $5 p^{5} 8 g$ & ${ }^{3} G^{o}$ & 5 & 472.68 & -5.502 & $9.41 \times 10^{4}$ \\
\hline $5 p^{5} 5 d$ & ${ }^{3} F^{o}$ & 3 & $5 p^{5} 8 g$ & ${ }^{3} G^{o}$ & 5 & 475.46 & -5.909 & $3.64 \times 10^{4}$ \\
\hline $5 p^{5} 5 d$ & ${ }^{3} D^{o}$ & 3 & $5 p^{5} 8 g$ & ${ }^{3} G^{o}$ & 5 & 495.36 & -5.105 & $2.13 \times 10^{5}$ \\
\hline $5 p^{5} 5 d$ & ${ }^{1} F^{o}$ & 3 & $5 p^{5} 8 g$ & ${ }^{3} G^{o}$ & 5 & 542.11 & -5.858 & $3.15 \times 10^{4}$ \\
\hline $5 p^{5} 5 d$ & ${ }^{3} F^{o}$ & 4 & $5 p^{5} 10 d$ & ${ }^{3} F^{o}$ & 4 & 472.67 & -6.136 & $2.18 \times 10^{4}$ \\
\hline $5 p^{5} 5 d$ & ${ }^{3} D^{o}$ & 3 & $5 p^{5} 10 d$ & ${ }^{3} F^{o}$ & 4 & 495.35 & -5.98 & $2.84 \times 10^{4}$ \\
\hline $5 p^{5} 5 d$ & ${ }^{3} D^{o}$ & 2 & $5 p^{5} 10 d$ & ${ }^{3} F^{o}$ & 4 & 535.05 & -6.069 & $1.99 \times 10^{4}$ \\
\hline $5 p^{5} 6 s$ & ${ }^{3} P^{o}$ & 2 & $5 p^{5} 10 d$ & ${ }^{3} F^{o}$ & 4 & 565.09 & -5.885 & $2.72 \times 10^{4}$ \\
\hline $5 p^{5} 5 d$ & ${ }^{3} F^{o}$ & 2 & $5 p^{5} 10 d$ & ${ }^{3} F^{o}$ & 3 & 482.76 & -6.031 & $2.67 \times 10^{4}$ \\
\hline $5 p^{5} 5 d$ & ${ }^{1} F^{o}$ & 3 & $5 p^{5} 10 d$ & ${ }^{3} F^{o}$ & 3 & 541.92 & -6.202 & $1.43 \times 10^{4}$ \\
\hline $5 p^{5} 5 d$ & ${ }^{1} P^{o}$ & 1 & $5 p^{5} 10 d$ & ${ }^{3} F^{o}$ & 3 & 627.33 & -6.062 & $1.47 \times 10^{4}$ \\
\hline $5 p^{5} 5 d$ & ${ }^{3} P^{o}$ & 2 & $5 p^{5} 10 d$ & ${ }^{3} D^{o}$ & 2 & 470.49 & -6.053 & $2.67 \times 10^{4}$ \\
\hline $5 p^{5} 5 d$ & ${ }^{3} D^{o}$ & 1 & $5 p^{5} 10 d$ & ${ }^{1} D^{o}$ & 2 & 513.88 & -5.642 & $5.76 \times 10^{4}$ \\
\hline $5 p^{5} 5 d$ & ${ }^{1} P^{o}$ & 1 & $5 p^{5} 10 d$ & ${ }^{1} D^{o}$ & 2 & 626.95 & -4.771 & $2.87 \times 10^{5}$ \\
\hline $5 p^{5} 6 s$ & ${ }^{3} P^{o}$ & 1 & $5 p^{5} 10 d$ & ${ }^{1} D^{o}$ & 2 & 645.54 & -6.165 & $1.10 \times 10^{4}$ \\
\hline $5 p^{5} 6 d$ & ${ }^{1} P^{o}$ & 1 & $5 p^{5} 10 d$ & ${ }^{1} D^{o}$ & 2 & 1180.62 & -5.642 & $1.09 \times 10^{4}$ \\
\hline $5 p^{5} 5 g$ & ${ }^{3} F^{o}$ & 2 & $5 p^{5} 8 i$ & ${ }^{3} H^{o}$ & 4 & 2247.55 & -4.974 & $1.40 \times 10^{4}$ \\
\hline $5 p^{5} 5 g$ & ${ }^{3} F^{o}$ & 3 & $5 p^{5} 8 i$ & ${ }^{3} H^{o}$ & 5 & 2251.17 & -4.659 & $2.89 \times 10^{4}$ \\
\hline $5 p^{5} 5 g$ & ${ }^{1} H^{o}$ & 5 & $5 p^{5} 8 i$ & ${ }^{1} K^{o}$ & 7 & 2260.76 & -4.428 & $4.87 \times 10^{4}$ \\
\hline $5 p^{5} 5 g$ & ${ }^{3} H^{o}$ & 6 & $5 p^{5} 8 i$ & ${ }^{3} K^{o}$ & 8 & 2258.9 & -4.364 & $5.66 \times 10^{4}$ \\
\hline $5 p^{5} 7 d$ & ${ }^{3} D^{o}$ & 3 & $5 p^{5} 8 i$ & ${ }^{3} I^{o}$ & 5 & 2281.15 & -5.041 & $1.17 \times 10^{4}$ \\
\hline $5 p^{5} 5 g$ & ${ }^{3} G^{o}$ & 3 & $5 p^{5} 8 i$ & ${ }^{3} I^{o}$ & 5 & 2290.01 & -4.888 & $1.65 \times 10^{4}$ \\
\hline $5 p^{5} 5 g$ & ${ }^{3} F^{o}$ & 4 & $5 p^{5} 8 i$ & ${ }^{3} H^{o}$ & 6 & 2282.77 & -4.554 & $3.57 \times 10^{4}$ \\
\hline $5 p^{5} 5 d$ & ${ }^{3} P^{o}$ & 1 & $5 p^{5} 10 d$ & ${ }^{3} D^{o}$ & 3 & 460.56 & -6.132 & $2.32 \times 10^{4}$ \\
\hline $5 p^{5} 5 d$ & ${ }^{3} P^{o}$ & 2 & $5 p^{5} 10 d$ & ${ }^{3} D^{o}$ & 3 & 470.25 & -5.844 & $4.32 \times 10^{4}$ \\
\hline $5 p^{5} 5 d$ & ${ }^{3} D^{\circ}$ & 3 & $5 p^{5} 10 d$ & ${ }^{3} D^{o}$ & 3 & 494.82 & -6.338 & $1.25 \times 10^{4}$ \\
\hline $5 p^{5} 5 d$ & ${ }^{3} D^{o}$ & 2 & $5 p^{5} 10 d$ & ${ }^{3} D^{o}$ & 3 & 534.44 & -5.977 & $2.46 \times 10^{4}$ \\
\hline $5 p^{5} 5 g$ & ${ }^{1} G^{o}$ & 4 & $5 p^{5} 8 i$ & ${ }^{1} I^{o}$ & 6 & 2295.3 & -4.538 & $3.67 \times 10^{4}$ \\
\hline $5 p^{5} 5 g$ & ${ }^{3} G^{o}$ & 5 & $5 p^{5} 8 i$ & ${ }^{3} I^{o}$ & 7 & 2296.02 & -4.462 & $4.37 \times 10^{4}$ \\
\hline $5 p^{5} 5 d$ & ${ }^{3} P^{o}$ & 0 & $5 p^{5} 9 g$ & ${ }^{3} F^{o}$ & 2 & 444.8 & -6.371 & $1.43 \times 10^{4}$ \\
\hline $5 p^{5} 5 d$ & ${ }^{3} F^{o}$ & 2 & $5 p^{5} 9 g$ & ${ }^{3} F^{o}$ & 2 & 470.14 & -6.128 & $2.25 \times 10^{4}$ \\
\hline $5 p^{5} 5 d$ & ${ }^{3} D^{o}$ & 1 & $5 p^{5} 9 g$ & ${ }^{3} F^{o}$ & 2 & 499.85 & -6.105 & $2.10 \times 10^{4}$ \\
\hline $5 p^{5} 6 s$ & ${ }^{1} P^{o}$ & 1 & $5 p^{5} 9 g$ & ${ }^{3} F^{o}$ & 2 & 553.29 & -6.164 & $1.49 \times 10^{4}$ \\
\hline $5 p^{5} 6 s$ & ${ }^{3} P^{o}$ & 0 & $5 p^{5} 9 g$ & ${ }^{3} F^{o}$ & 2 & 618.84 & -5.839 & $2.52 \times 10^{4}$ \\
\hline $5 p^{5} 6 s$ & ${ }^{3} P^{o}$ & 1 & $5 p^{5} 9 g$ & ${ }^{3} F^{o}$ & 2 & 623.54 & -6.052 & $1.52 \times 10^{4}$ \\
\hline $5 p^{5} 5 d$ & ${ }^{3} F^{o}$ & 3 & $5 p^{5} 9 g$ & ${ }^{3} F^{o}$ & 3 & 463.02 & -6.375 & $1.31 \times 10^{4}$ \\
\hline $5 p^{5} 5 d$ & ${ }^{3} D^{o}$ & 3 & $5 p^{5} 9 g$ & ${ }^{3} F^{o}$ & 3 & 481.87 & -6.309 & $1.41 \times 10^{4}$ \\
\hline
\end{tabular}


TABLE VI (cont.)

\begin{tabular}{|c|c|c|c|c|c|c|c|c|}
\hline \multicolumn{3}{|c|}{ Lower Level } & \multicolumn{3}{|c|}{ Upper Level } & $\lambda[\AA]$ & $\log (g f)$ & $g A_{j i}\left[\mathrm{~s}^{-1}\right]$ \\
\hline Conf. & Term & $J$ & Conf. & Term & $J$ & HFR & HFR & HFR \\
\hline $5 p^{5} 5 d$ & ${ }^{3} D^{o}$ & 1 & $5 p^{5} 9 g$ & ${ }^{3} F^{o}$ & 3 & 499.78 & -5.359 & $1.17 \times 10^{5}$ \\
\hline $5 p^{5} 5 d$ & ${ }^{3} D^{o}$ & 2 & $5 p^{5} 9 g$ & ${ }^{3} F^{o}$ & 3 & 519.36 & -5.878 & $3.27 \times 10^{4}$ \\
\hline $5 p^{5} 6 s$ & ${ }^{3} P^{o}$ & 2 & $5 p^{5} 9 g$ & ${ }^{3} F^{o}$ & 3 & 547.61 & -5.86 & $3.07 \times 10^{4}$ \\
\hline $5 p^{5} 6 s$ & ${ }^{1} P^{o}$ & 1 & $5 p^{5} 9 g$ & ${ }^{3} F^{o}$ & 3 & 553.2 & -6.289 & $1.12 \times 10^{4}$ \\
\hline $5 p^{5} 5 d$ & ${ }^{1} P^{o}$ & 1 & $5 p^{5} 9 g$ & ${ }^{3} F^{o}$ & 3 & 606.08 & -4.275 & $9.65 \times 10^{5}$ \\
\hline $5 p^{5} 6 s$ & ${ }^{3} P^{o}$ & 1 & $5 p^{5} 9 g$ & ${ }^{3} F^{o}$ & 3 & 623.44 & -5.483 & $5.64 \times 10^{4}$ \\
\hline $5 p^{5} 6 d$ & ${ }^{1} P^{o}$ & 1 & $5 p^{5} 9 g$ & ${ }^{3} F^{o}$ & 3 & 1108.74 & -5.096 & $4.35 \times 10^{4}$ \\
\hline $5 p^{5} 6 d$ & ${ }^{3} D^{o}$ & 1 & $5 p^{5} 9 g$ & ${ }^{3} F^{o}$ & 3 & 1407.32 & -5.373 & $1.43 \times 10^{4}$ \\
\hline $5 p^{5} 5 d$ & ${ }^{3} F^{o}$ & 3 & $5 p^{5} 9 g$ & ${ }^{1} H^{o}$ & 5 & 462.97 & -5.494 & $9.99 \times 10^{4}$ \\
\hline $5 p^{5} 5 d$ & ${ }^{3} D^{o}$ & 3 & $5 p^{5} 9 g$ & ${ }^{1} H^{o}$ & 5 & 481.81 & -5.338 & $1.32 \times 10^{5}$ \\
\hline $5 p^{5} 5 d$ & ${ }^{1} F^{o}$ & 3 & $5 p^{5} 9 g$ & ${ }^{1} H^{o}$ & 5 & 525.92 & -5.236 & $1.40 \times 10^{5}$ \\
\hline $5 p^{5} 5 d$ & ${ }^{3} F^{o}$ & 4 & $5 p^{5} 9 g$ & ${ }^{3} F^{o}$ & 4 & 460.17 & -6.363 & $1.37 \times 10^{4}$ \\
\hline $5 p^{5} 5 d$ & ${ }^{3} D^{o}$ & 3 & $5 p^{5} 9 g$ & ${ }^{3} F^{o}$ & 4 & 481.64 & -5.486 & $9.40 \times 10^{4}$ \\
\hline $5 p^{5} 5 d$ & ${ }^{3} D^{o}$ & 2 & $5 p^{5} 9 g$ & ${ }^{3} F^{o}$ & 4 & 519.09 & -5.557 & $6.87 \times 10^{4}$ \\
\hline $5 p^{5} 6 s$ & ${ }^{3} P^{o}$ & 2 & $5 p^{5} 9 g$ & ${ }^{3} F^{o}$ & 4 & 547.31 & -5.503 & $6.99 \times 10^{4}$ \\
\hline $5 p^{5} 5 d$ & ${ }^{3} F^{o}$ & 2 & $5 p^{5} 9 g$ & ${ }^{3} G^{o}$ & 3 & 469.81 & -6.198 & $1.92 \times 10^{4}$ \\
\hline $5 p^{5} 5 d$ & ${ }^{3} D^{o}$ & 1 & $5 p^{5} 9 g$ & ${ }^{3} G^{o}$ & 3 & 499.47 & -4.817 & $4.07 \times 10^{5}$ \\
\hline $5 p^{5} 5 d$ & ${ }^{3} F^{o}$ & 2 & $5 p^{5} 9 g$ & ${ }^{3} G^{o}$ & 3 & 510.11 & -5.938 & $2.95 \times 10^{4}$ \\
\hline $5 p^{5} 5 d$ & ${ }^{1} P^{o}$ & 1 & $5 p^{5} 9 g$ & ${ }^{3} G^{o}$ & 3 & 605.64 & -4.196 & $1.16 \times 10^{6}$ \\
\hline $5 p^{5} 6 d$ & ${ }^{1} P^{o}$ & 1 & $5 p^{5} 9 g$ & ${ }^{3} G^{o}$ & 3 & 1107.24 & -4.836 & $7.94 \times 10^{4}$ \\
\hline $5 p^{5} 5 d$ & ${ }^{3} F^{o}$ & 3 & $5 p^{5} 9 g$ & ${ }^{1} G^{o}$ & 4 & 462.71 & -6.387 & $1.28 \times 10^{4}$ \\
\hline $5 p^{5} 5 d$ & ${ }^{3} F^{o}$ & 2 & $5 p^{5} 9 g$ & ${ }^{1} G^{o}$ & 4 & 469.76 & -5.4 & $1.20 \times 10^{5}$ \\
\hline $5 p^{5} 5 d$ & ${ }^{3} F^{o}$ & 2 & $5 p^{5} 9 g$ & ${ }^{1} G^{o}$ & 4 & 510.05 & -5.702 & $5.10 \times 10^{4}$ \\
\hline $5 p^{5} 5 d$ & ${ }^{1} F^{o}$ & 3 & $5 p^{5} 9 g$ & ${ }^{1} G^{o}$ & 4 & 525.6 & -5.855 & $3.37 \times 10^{4}$ \\
\hline $5 p^{5} 6 d$ & ${ }^{1} D^{o}$ & 2 & $5 p^{5} 9 g$ & ${ }^{1} G^{o}$ & 4 & 1067.03 & -5.703 & $1.16 \times 10^{4}$ \\
\hline $5 p^{5} 5 d$ & ${ }^{3} D^{o}$ & 3 & $5 p^{5} 9 g$ & ${ }^{3} G^{o}$ & 5 & 481.53 & -4.921 & $3.45 \times 10^{5}$ \\
\hline $5 p^{5} 6 d$ & ${ }^{3} D^{o}$ & 3 & $5 p^{5} 9 g$ & ${ }^{3} G^{o}$ & 5 & 1072.45 & -5.437 & $2.12 \times 10^{4}$ \\
\hline $5 p^{5} 5 g$ & ${ }^{3} F^{o}$ & 2 & $5 p^{5} 9 i$ & ${ }^{3} H^{o}$ & 4 & 1989.36 & -5.213 & $1.03 \times 10^{4}$ \\
\hline $5 p^{5} 5 g$ & ${ }^{3} F^{o}$ & 3 & $5 p^{5} 9 i$ & ${ }^{3} H^{o}$ & 5 & 1992.19 & -4.9 & $2.12 \times 10^{4}$ \\
\hline $5 p^{5} 5 g$ & ${ }^{1} H^{o}$ & 5 & $5 p^{5} 9 i$ & ${ }^{1} K^{o}$ & 7 & 1999.84 & -4.672 & $3.55 \times 10^{4}$ \\
\hline $5 p^{5} 5 g$ & ${ }^{3} H^{o}$ & 6 & $5 p^{5} 9 i$ & ${ }^{3} K^{o}$ & 8 & 1998.39 & -4.606 & $4.14 \times 10^{4}$ \\
\hline $5 p^{5} 5 g$ & ${ }^{3} G^{o}$ & 3 & $5 p^{5} 9 i$ & ${ }^{3} I^{o}$ & 5 & 2023.22 & -5.14 & $1.18 \times 10^{4}$ \\
\hline $5 p^{5} 5 g$ & ${ }^{3} F^{o}$ & 4 & $5 p^{5} 9 i$ & ${ }^{3} H^{o}$ & 6 & 2017.57 & -4.802 & $2.59 \times 10^{4}$ \\
\hline $5 p^{5} 5 g$ & ${ }^{1} G^{o}$ & 4 & $5 p^{5} 9 i$ & ${ }^{1} I^{o}$ & 6 & 2027.51 & -4.787 & $2.65 \times 10^{4}$ \\
\hline $5 p^{5} 5 g$ & ${ }^{3} G^{o}$ & 5 & $5 p^{5} 9 i$ & ${ }^{3} I^{o}$ & 7 & 2028.07 & -4.712 & $3.15 \times 10^{4}$ \\
\hline $5 p^{5} 5 d$ & ${ }^{3} P^{o}$ & 0 & $5 p^{5} 10 g$ & ${ }^{3} F^{o}$ & 2 & 436.62 & -6.169 & $2.37 \times 10^{4}$ \\
\hline $5 p^{5} 5 d$ & ${ }^{3} P^{o}$ & 1 & $5 p^{5} 10 g$ & ${ }^{3} F^{o}$ & 2 & 441.02 & -6.054 & $3.03 \times 10^{4}$ \\
\hline $5 p^{5} 5 d$ & ${ }^{3} P^{o}$ & 2 & $5 p^{5} 10 g$ & ${ }^{3} F^{o}$ & 2 & 449.89 & -6.236 & $1.92 \times 10^{4}$ \\
\hline $5 p^{5} 5 d$ & ${ }^{3} F^{o}$ & 3 & $5 p^{5} 10 g$ & ${ }^{3} F^{o}$ & 2 & 454.21 & -5.399 & $1.29 \times 10^{5}$ \\
\hline $5 p^{5} 5 d$ & ${ }^{3} F^{o}$ & 2 & $5 p^{5} 10 g$ & ${ }^{3} F^{o}$ & 2 & 461 & -5.234 & $1.83 \times 10^{5}$ \\
\hline $5 p^{5} 5 d$ & ${ }^{3} F^{o}$ & 2 & $5 p^{5} 10 g$ & ${ }^{3} F^{o}$ & 2 & 499.74 & -5.297 & $1.35 \times 10^{5}$ \\
\hline $5 p^{5} 5 d$ & ${ }^{1} F^{o}$ & 3 & $5 p^{5} 10 g$ & ${ }^{3} F^{o}$ & 2 & 514.66 & -5.993 & $2.56 \times 10^{4}$ \\
\hline $5 p^{5} 5 d$ & ${ }^{3} P^{o}$ & 1 & $5 p^{5} 10 g$ & ${ }^{3} F^{o}$ & 3 & 440.98 & -6.024 & $3.25 \times 10^{4}$ \\
\hline $5 p^{5} 5 d$ & ${ }^{3} P^{o}$ & 2 & $5 p^{5} 10 g$ & ${ }^{3} F^{o}$ & 3 & 449.86 & -6.18 & $2.18 \times 10^{4}$ \\
\hline $5 p^{5} 5 d$ & ${ }^{3} F^{o}$ & 4 & $5 p^{5} 10 g$ & ${ }^{3} F^{o}$ & 3 & 451.63 & -5.437 & $1.20 \times 10^{5}$ \\
\hline $5 p^{5} 5 d$ & ${ }^{3} F^{o}$ & 3 & $5 p^{5} 10 g$ & ${ }^{3} F^{o}$ & 3 & 454.18 & -5.214 & $1.98 \times 10^{5}$ \\
\hline $5 p^{5} 5 d$ & ${ }^{3} F^{o}$ & 2 & $5 p^{5} 10 g$ & ${ }^{3} F^{o}$ & 3 & 460.96 & -5.68 & $6.55 \times 10^{4}$ \\
\hline $5 p^{5} 5 d$ & ${ }^{3} D^{o}$ & 3 & $5 p^{5} 10 g$ & ${ }^{3} F^{o}$ & 3 & 472.3 & -6.325 & $1.42 \times 10^{4}$ \\
\hline $5 p^{5} 5 d$ & ${ }^{3} D^{o}$ & 1 & $5 p^{5} 10 g$ & ${ }^{3} F^{o}$ & 3 & 489.49 & -6.297 & $1.41 \times 10^{4}$ \\
\hline $5 p^{5} 5 d$ & ${ }^{3} F^{o}$ & 2 & $5 p^{5} 10 g$ & ${ }^{3} F^{o}$ & 3 & 499.7 & -5.68 & $5.58 \times 10^{4}$ \\
\hline $5 p^{5} 5 d$ & ${ }^{1} F^{o}$ & 3 & $5 p^{5} 10 g$ & ${ }^{3} F^{o}$ & 3 & 514.61 & -5.631 & $5.90 \times 10^{4}$ \\
\hline $5 p^{5} 5 d$ & ${ }^{1} P^{o}$ & 1 & $5 p^{5} 10 g$ & ${ }^{3} F^{o}$ & 3 & 591.02 & -5.842 & $2.75 \times 10^{4}$ \\
\hline
\end{tabular}


TABLE VI (cont.)

\begin{tabular}{|c|c|c|c|c|c|c|c|c|}
\hline \multicolumn{3}{|c|}{ Lower Level } & \multicolumn{3}{|c|}{ Upper Level } & $\lambda[\AA]$ & $\log (g f)$ & $g A_{j i}\left[\mathrm{~s}^{-1}\right]$ \\
\hline Conf. & Term & $J$ & Conf. & Term & $\bar{J}$ & HFR & HFR & $\mathrm{HFR}$ \\
\hline $5 p^{5} 5 d$ & ${ }^{3} F^{o}$ & 4 & $5 p^{5} 10 g$ & ${ }^{3} H^{o}$ & 6 & 451.61 & -5.116 & $2.50 \times 10^{5}$ \\
\hline $5 p^{5} 6 d$ & ${ }^{3} F^{o}$ & 4 & $5 p^{5} 10 g$ & ${ }^{3} H^{o}$ & 6 & 1006.71 & -5.813 & $1.01 \times 10^{4}$ \\
\hline $5 p^{5} 5 d$ & ${ }^{3} F^{o}$ & 3 & $5 p^{5} 10 g$ & ${ }^{1} H^{o}$ & 5 & 454.13 & -5.258 & $1.79 \times 10^{5}$ \\
\hline $5 p^{5} 5 d$ & ${ }^{3} D^{o}$ & 3 & $5 p^{5} 10 g$ & ${ }^{1} H^{o}$ & 5 & 472.25 & -5.937 & $3.46 \times 10^{4}$ \\
\hline $5 p^{5} 5 d$ & ${ }^{3} P^{o}$ & 2 & $5 p^{5} 10 g$ & ${ }^{3} F^{o}$ & 4 & 449.72 & -5.589 & $8.49 \times 10^{4}$ \\
\hline $5 p^{5} 5 d$ & ${ }^{3} F^{o}$ & 4 & $5 p^{5} 10 g$ & ${ }^{3} F^{o}$ & 4 & 451.49 & -5.052 & $2.90 \times 10^{5}$ \\
\hline $5 p^{5} 5 d$ & ${ }^{3} F^{o}$ & 3 & $5 p^{5} 10 g$ & ${ }^{3} F^{o}$ & 4 & 454.03 & -5.561 & $8.88 \times 10^{4}$ \\
\hline $5 p^{5} 5 d$ & ${ }^{3} D^{o}$ & 3 & $5 p^{5} 10 g$ & ${ }^{3} F^{o}$ & 4 & 472.14 & -5.913 & $3.65 \times 10^{4}$ \\
\hline $5 p^{5} 5 d$ & ${ }^{3} F^{o}$ & 2 & $5 p^{5} 10 g$ & ${ }^{3} F^{o}$ & 4 & 499.53 & -6.293 & $1.36 \times 10^{4}$ \\
\hline $5 p^{5} 5 d$ & ${ }^{1} F^{o}$ & 3 & $5 p^{5} 10 g$ & ${ }^{3} F^{o}$ & 4 & 514.43 & -5.693 & $5.11 \times 10^{4}$ \\
\hline $5 p^{5} 5 d$ & ${ }^{3} P^{o}$ & 1 & $5 p^{5} 10 g$ & ${ }^{3} G^{o}$ & 3 & 440.81 & -6.521 & $1.03 \times 10^{4}$ \\
\hline $5 p^{5} 5 d$ & ${ }^{3} F^{o}$ & 3 & $5 p^{5} 10 g$ & ${ }^{3} G^{o}$ & 3 & 453.99 & -6.203 & $2.03 \times 10^{4}$ \\
\hline $5 p^{5} 5 d$ & ${ }^{3} D^{o}$ & 1 & $5 p^{5} 10 g$ & ${ }^{3} G^{o}$ & 3 & 489.28 & -5.673 & $5.91 \times 10^{4}$ \\
\hline $5 p^{5} 5 d$ & ${ }^{3} F^{o}$ & 2 & $5 p^{5} 10 g$ & ${ }^{3} G^{o}$ & 3 & 499.48 & -5.588 & $6.90 \times 10^{4}$ \\
\hline $5 p^{5} 5 d$ & ${ }^{1} P^{o}$ & 1 & $5 p^{5} 10 g$ & ${ }^{3} G^{o}$ & 3 & 590.71 & -5.589 & $4.93 \times 10^{4}$ \\
\hline $5 p^{5} 5 d$ & ${ }^{3} F^{o}$ & 2 & $5 p^{5} 10 g$ & ${ }^{1} G^{o}$ & 4 & 460.74 & -5.27 & $1.69 \times 10^{5}$ \\
\hline $5 p^{5} 5 d$ & ${ }^{3} F^{o}$ & 4 & $5 p^{5} 10 g$ & ${ }^{3} G^{o}$ & 5 & 451.41 & -6.06 & $2.85 \times 10^{4}$ \\
\hline $5 p^{5} 5 d$ & ${ }^{3} F^{o}$ & 3 & $5 p^{5} 10 g$ & ${ }^{3} G^{o}$ & 5 & 453.95 & -5.801 & $5.12 \times 10^{4}$ \\
\hline $5 p^{5} 5 d$ & ${ }^{3} D^{o}$ & 3 & $5 p^{5} 10 g$ & ${ }^{3} G^{o}$ & 5 & 472.06 & -5.255 & $1.67 \times 10^{5}$ \\
\hline $5 p^{5} 5 d$ & ${ }^{3} F^{o}$ & 4 & $5 p^{5} 10 i$ & ${ }^{3} H^{o}$ & 5 & 451.21 & -6.402 & $1.30 \times 10^{4}$ \\
\hline $5 p^{5} 5 d$ & ${ }^{3} F^{o}$ & 3 & $5 p^{5} 10 i$ & ${ }^{3} H^{o}$ & 5 & 453.75 & -5.623 & $7.71 \times 10^{4}$ \\
\hline $5 p^{5} 5 d$ & ${ }^{3} F^{o}$ & 3 & $5 p^{5} 10 i$ & ${ }^{3} H^{o}$ & 4 & 453.75 & -6.253 & $1.81 \times 10^{4}$ \\
\hline $5 p^{5} 5 d$ & ${ }^{3} F^{o}$ & 2 & $5 p^{5} 10 i$ & ${ }^{3} H^{o}$ & 4 & 460.52 & -5.625 & $7.45 \times 10^{4}$ \\
\hline $5 p^{5} 5 d$ & ${ }^{3} F^{o}$ & 2 & $5 p^{5} 10 i$ & ${ }^{3} H^{o}$ & 4 & 499.18 & -5.588 & $6.91 \times 10^{4}$ \\
\hline $5 p^{5} 5 d$ & ${ }^{1} F^{o}$ & 3 & $5 p^{5} 10 i$ & ${ }^{3} H^{o}$ & 5 & 514.06 & -5.931 & $2.96 \times 10^{4}$ \\
\hline $5 p^{5} 5 g$ & ${ }^{3} F^{o}$ & 3 & $5 p^{5} 10 i$ & ${ }^{3} H^{o}$ & 5 & 1840.8 & -5.078 & $1.64 \times 10^{4}$ \\
\hline $5 p^{5} 5 g$ & ${ }^{3} H^{o}$ & 6 & $5 p^{5} 10 i$ & ${ }^{3} K^{o}$ & 8 & 1846.17 & -4.785 & $3.21 \times 10^{4}$ \\
\hline $5 p^{5} 5 g$ & ${ }^{1} H^{o}$ & 5 & $5 p^{5} 10 i$ & ${ }^{1} K^{o}$ & 7 & 1847.38 & -4.858 & $2.71 \times 10^{4}$ \\
\hline $5 p^{5} 5 d$ & ${ }^{3} F^{o}$ & 4 & $5 p^{5} 10 i$ & ${ }^{3} H^{o}$ & 6 & 451.15 & -5.454 & $1.15 \times 10^{5}$ \\
\hline $5 p^{5} 5 d$ & ${ }^{3} F^{o}$ & 3 & $5 p^{5} 10 i$ & ${ }^{3} I^{o}$ & 5 & 453.69 & -5.971 & $3.46 \times 10^{4}$ \\
\hline $5 p^{5} 5 g$ & ${ }^{3} F^{o}$ & 4 & $5 p^{5} 10 i$ & ${ }^{3} H^{o}$ & 6 & 1862.81 & -5.019 & $1.84 \times 10^{4}$ \\
\hline $5 p^{5} 5 g$ & ${ }^{1} G^{o}$ & 4 & $5 p^{5} 10 i$ & ${ }^{1} I^{o}$ & 6 & 1871.35 & -5.019 & $1.83 \times 10^{4}$ \\
\hline $5 p^{5} 5 g$ & ${ }^{3} G^{o}$ & 5 & $5 p^{5} 10 i$ & ${ }^{3} I^{o}$ & 7 & 1871.84 & -4.945 & $2.16 \times 10^{4}$ \\
\hline $5 p^{5} 5 d$ & ${ }^{3} F^{o}$ & 2 & $5 p^{5} 7 g$ & ${ }^{3} H^{o}$ & 4 & 455.33 & -6.166 & $2.19 \times 10^{4}$ \\
\hline $5 p^{5} 5 d$ & ${ }^{3} F^{o}$ & 2 & $5 p^{5} 7 g$ & ${ }^{3} H^{o}$ & 4 & 493.09 & -4.814 & $4.21 \times 10^{5}$ \\
\hline $5 p^{5} 5 d$ & ${ }^{1} F^{o}$ & 3 & $5 p^{5} 7 g$ & ${ }^{3} H^{o}$ & 4 & 507.6 & -5.885 & $3.37 \times 10^{4}$ \\
\hline $5 p^{5} 5 d$ & ${ }^{3} P^{o}$ & 2 & $5 p^{5} 7 g$ & ${ }^{3} F^{o}$ & 4 & 444.47 & -5.914 & $4.12 \times 10^{4}$ \\
\hline $5 p^{5} 5 d$ & ${ }^{3} D^{o}$ & 2 & $5 p^{5} 7 g$ & ${ }^{3} F^{o}$ & 4 & 501.39 & -4.854 & $3.72 \times 10^{5}$ \\
\hline $5 p^{5} 5 d$ & ${ }^{1} F^{o}$ & 3 & $5 p^{5} 7 g$ & ${ }^{3} F^{o}$ & 4 & 507.57 & -5.895 & $3.30 \times 10^{4}$ \\
\hline $5 p^{5} 6 s$ & ${ }^{3} P^{o}$ & 2 & $5 p^{5} 7 g$ & ${ }^{3} F^{o}$ & 4 & 527.67 & -6.364 & $1.04 \times 10^{4}$ \\
\hline $5 p^{5} 5 d$ & ${ }^{3} F^{o}$ & 3 & $5 p^{5} 7 g$ & ${ }^{3} H^{o}$ & 5 & 448.68 & -6.1 & $2.63 \times 10^{4}$ \\
\hline $5 p^{5} 5 d$ & ${ }^{1} F^{o}$ & 3 & $5 p^{5} 7 g$ & ${ }^{3} H^{o}$ & 5 & 507.56 & -4.726 & $4.87 \times 10^{5}$ \\
\hline $5 p^{5} 5 d$ & ${ }^{3} P^{o}$ & 1 & $5 p^{5} 7 g$ & ${ }^{3} G^{o}$ & 3 & 435.74 & -6.357 & $1.54 \times 10^{4}$ \\
\hline $5 p^{5} 5 d$ & ${ }^{3} D^{o}$ & 1 & $5 p^{5} 7 g$ & ${ }^{3} G^{o}$ & 3 & 483.03 & -5.306 & $1.41 \times 10^{5}$ \\
\hline $5 p^{5} 5 d$ & ${ }^{3} F^{o}$ & 2 & $5 p^{5} 7 g$ & ${ }^{3} G^{o}$ & 3 & 492.97 & -5.982 & $2.86 \times 10^{4}$ \\
\hline $5 p^{5} 5 d$ & ${ }^{3} D^{o}$ & 2 & $5 p^{5} 7 g$ & ${ }^{3} G^{o}$ & 3 & 501.3 & -5.921 & $3.18 \times 10^{4}$ \\
\hline $5 p^{5} 5 d$ & ${ }^{1} P^{o}$ & 1 & $5 p^{5} 7 g$ & ${ }^{3} G^{o}$ & 3 & 581.63 & -5.487 & $6.43 \times 10^{4}$ \\
\hline $5 p^{5} 5 d$ & ${ }^{1} F^{o}$ & 3 & $5 p^{5} 9 d$ & ${ }^{3} F^{o}$ & 3 & 506.92 & -6.413 & $1.00 \times 10^{4}$ \\
\hline $5 p^{5} 5 g$ & ${ }^{3} H^{o}$ & 4 & $5 p^{5} 7 i$ & ${ }^{3} K^{o}$ & 6 & 2801.47 & -4.243 & $4.86 \times 10^{4}$ \\
\hline $5 p^{5} 5 g$ & ${ }^{3} G^{o}$ & 3 & $5 p^{5} 7 i$ & ${ }^{3} I^{o}$ & 5 & 2801.69 & -4.391 & $3.46 \times 10^{4}$ \\
\hline $5 p^{5} 5 g$ & ${ }^{3} H^{o}$ & 5 & $5 p^{5} 7 i$ & ${ }^{3} K^{o}$ & 7 & 2803.94 & -4.164 & $5.81 \times 10^{4}$ \\
\hline $5 p^{5} 5 g$ & ${ }^{3} F^{o}$ & 4 & $5 p^{5} 7 i$ & ${ }^{3} H^{o}$ & 6 & 2796.88 & -4.292 & $4.36 \times 10^{4}$ \\
\hline
\end{tabular}


TABLE VI (cont.)

\begin{tabular}{|c|c|c|c|c|c|c|c|c|}
\hline \multicolumn{3}{|c|}{ Lower Level } & \multicolumn{3}{|c|}{ Upper Level } & \multirow{2}{*}{$\begin{array}{c}\lambda[\AA] \\
\mathrm{HFR}\end{array}$} & \multirow{2}{*}{$\frac{\log (g f)}{\mathrm{HFR}}$} & \multirow{2}{*}{$\frac{g A_{j i}\left[\mathrm{~s}^{-1}\right]}{\mathrm{HFR}}$} \\
\hline Conf. & Term & $J$ & Conf. & Term & $J$ & & & \\
\hline $5 p^{5} 5 d$ & ${ }^{1} P^{o}$ & 1 & $5 p^{5} 9 d$ & ${ }^{3} D^{o}$ & 1 & 579.77 & -6.119 & $1.51 \times 10^{4}$ \\
\hline $5 p^{5} 5 d$ & ${ }^{1} P^{o}$ & 1 & $5 p^{5} 10 s$ & ${ }^{3} P^{o}$ & 1 & 579.02 & -6.214 & $1.22 \times 10^{4}$ \\
\hline $5 p^{5} 5 d$ & ${ }^{3} F^{o}$ & 2 & $5 p^{5} 8 g$ & ${ }^{3} H^{o}$ & 4 & 438.26 & -5.914 & $4.23 \times 10^{4}$ \\
\hline $5 p^{5} 5 d$ & ${ }^{3} F^{o}$ & 2 & $5 p^{5} 8 g$ & ${ }^{3} H^{o}$ & 4 & 473.12 & -4.922 & $3.56 \times 10^{5}$ \\
\hline $5 p^{5} 5 d$ & ${ }^{1} F^{o}$ & 3 & $5 p^{5} 8 g$ & ${ }^{3} H^{o}$ & 4 & 486.47 & -6.075 & $2.37 \times 10^{4}$ \\
\hline $5 p^{5} 6 d$ & ${ }^{3} F^{o}$ & 2 & $5 p^{5} 8 g$ & ${ }^{3} H^{o}$ & 4 & 1121.84 & -5.688 & $1.09 \times 10^{4}$ \\
\hline $5 p^{5} 5 d$ & ${ }^{3} P^{o}$ & 2 & $5 p^{5} 8 g$ & ${ }^{3} F^{o}$ & 4 & 428.19 & -5.624 & $8.65 \times 10^{4}$ \\
\hline $5 p^{5} 5 d$ & ${ }^{3} D^{o}$ & 2 & $5 p^{5} 8 g$ & ${ }^{3} F^{o}$ & 4 & 480.77 & -5.002 & $2.87 \times 10^{5}$ \\
\hline $5 p^{5} 5 d$ & ${ }^{1} F^{o}$ & 3 & $5 p^{5} 8 g$ & ${ }^{3} F^{o}$ & 4 & 486.45 & -6.035 & $2.60 \times 10^{4}$ \\
\hline $5 p^{5} 6 s$ & ${ }^{3} P^{o}$ & 2 & $5 p^{5} 8 g$ & ${ }^{3} F^{o}$ & 4 & 504.88 & -5.999 & $2.62 \times 10^{4}$ \\
\hline $5 p^{5} 6 d$ & ${ }^{3} P^{o}$ & 2 & $5 p^{5} 8 g$ & ${ }^{3} F^{o}$ & 4 & 1127.47 & -5.697 & $1.06 \times 10^{4}$ \\
\hline $5 p^{5} 5 d$ & ${ }^{3} F^{o}$ & 3 & $5 p^{5} 8 g$ & ${ }^{3} H^{o}$ & 5 & 432.09 & -5.797 & $5.70 \times 10^{4}$ \\
\hline $5 p^{5} 5 d$ & ${ }^{1} F^{o}$ & 3 & $5 p^{5} 8 g$ & ${ }^{3} H^{o}$ & 5 & 486.44 & -4.916 & $3.42 \times 10^{5}$ \\
\hline $5 p^{5} 6 d$ & ${ }^{3} F^{o}$ & 3 & $5 p^{5} 8 g$ & ${ }^{3} H^{o}$ & 5 & 1131.01 & -5.606 & $1.29 \times 10^{4}$ \\
\hline $5 p^{5} 5 d$ & ${ }^{3} P^{o}$ & 1 & $5 p^{5} 8 g$ & ${ }^{3} G^{o}$ & 3 & 420.09 & -6.223 & $2.26 \times 10^{4}$ \\
\hline $5 p^{5} 5 d$ & ${ }^{3} P^{o}$ & 2 & $5 p^{5} 8 g$ & ${ }^{3} G^{o}$ & 3 & 428.13 & -6.56 & $1.00 \times 10^{4}$ \\
\hline $5 p^{5} 5 d$ & ${ }^{3} D^{o}$ & 1 & $5 p^{5} 8 g$ & ${ }^{3} G^{o}$ & 3 & 463.88 & -5.319 & $1.49 \times 10^{5}$ \\
\hline $5 p^{5} 5 d$ & ${ }^{3} F^{o}$ & 2 & $5 p^{5} 8 g$ & ${ }^{3} G^{o}$ & 3 & 473.03 & -5.996 & $3.01 \times 10^{4}$ \\
\hline $5 p^{5} 5 d$ & ${ }^{3} D^{o}$ & 2 & $5 p^{5} 8 g$ & ${ }^{3} G^{o}$ & 3 & 480.7 & -6.058 & $2.53 \times 10^{4}$ \\
\hline $5 p^{5} 5 d$ & ${ }^{3} P^{o}$ & 0 & $5 p^{5} 10 d$ & ${ }^{3} F^{o}$ & 2 & 415.96 & -6.253 & $2.15 \times 10^{4}$ \\
\hline $5 p^{5} 6 s$ & ${ }^{1} P^{o}$ & 1 & $5 p^{5} 10 d$ & ${ }^{3} F^{o}$ & 2 & 509.35 & -6.405 & $1.01 \times 10^{4}$ \\
\hline $5 p^{5} 5 d$ & ${ }^{1} P^{o}$ & 1 & $5 p^{5} 10 d$ & ${ }^{3} F^{o}$ & 2 & 553.84 & -5.164 & $1.49 \times 10^{5}$ \\
\hline $5 p^{5} 5 d$ & ${ }^{3} P^{o}$ & 0 & $5 p^{5} 10 d$ & ${ }^{3} P^{o}$ & 2 & 415.83 & -6.103 & $3.04 \times 10^{4}$ \\
\hline $5 p^{5} 5 d$ & ${ }^{3} P^{o}$ & 2 & $5 p^{5} 10 d$ & ${ }^{3} P^{o}$ & 2 & 427.86 & -6.132 & $2.69 \times 10^{4}$ \\
\hline $5 p^{5} 6 s$ & ${ }^{3} P^{o}$ & 2 & $5 p^{5} 10 d$ & ${ }^{3} P^{o}$ & 2 & 504.42 & -6.262 & $1.44 \times 10^{4}$ \\
\hline $5 p^{5} 5 d$ & ${ }^{1} P^{o}$ & 1 & $5 p^{5} 10 d$ & ${ }^{3} P^{o}$ & 2 & 553.62 & -5.309 & $1.07 \times 10^{5}$ \\
\hline $5 p^{5} 5 d$ & ${ }^{3} P^{o}$ & 1 & $5 p^{5} 10 d$ & ${ }^{3} F^{o}$ & 3 & 419.75 & -5.75 & $6.73 \times 10^{4}$ \\
\hline $5 p^{5} 5 d$ & ${ }^{3} F^{o}$ & 3 & $5 p^{5} 10 d$ & ${ }^{3} F^{o}$ & 3 & 431.68 & -6.129 & $2.66 \times 10^{4}$ \\
\hline $5 p^{5} 5 g$ & ${ }^{3} H^{o}$ & 4 & $5 p^{5} 8 i$ & ${ }^{3} K^{o}$ & 6 & 2267.83 & -4.525 & $3.87 \times 10^{4}$ \\
\hline $5 p^{5} 5 g$ & ${ }^{3} G^{o}$ & 3 & $5 p^{5} 8 i$ & ${ }^{3} I^{o}$ & 5 & 2267.99 & -4.672 & $2.76 \times 10^{4}$ \\
\hline $5 p^{5} 5 g$ & ${ }^{3} H^{o}$ & 5 & $5 p^{5} 8 i$ & ${ }^{3} K^{o}$ & 7 & 2269.45 & -4.447 & $4.63 \times 10^{4}$ \\
\hline $5 p^{5} 5 g$ & ${ }^{3} F^{o}$ & 4 & $5 p^{5} 8 i$ & ${ }^{3} H^{o}$ & 6 & 2264.83 & -4.571 & $3.49 \times 10^{4}$ \\
\hline $5 p^{5} 5 d$ & ${ }^{3} P^{o}$ & 1 & $5 p^{5} 10 d$ & ${ }^{3} D^{o}$ & 1 & 419.34 & -5.925 & $4.51 \times 10^{4}$ \\
\hline $5 p^{5} 5 d$ & ${ }^{1} P^{o}$ & 1 & $5 p^{5} 10 d$ & ${ }^{3} D^{o}$ & 1 & 552.79 & -5.448 & $7.78 \times 10^{4}$ \\
\hline $5 p^{5} 5 d$ & ${ }^{3} F^{o}$ & 2 & $5 p^{5} 9 g$ & ${ }^{3} H^{o}$ & 4 & 427.28 & -5.985 & $3.78 \times 10^{4}$ \\
\hline $5 p^{5} 5 d$ & ${ }^{3} F^{o}$ & 2 & $5 p^{5} 9 g$ & ${ }^{3} H^{o}$ & 4 & 460.35 & -5.862 & $4.33 \times 10^{4}$ \\
\hline $5 p^{5} 5 d$ & ${ }^{1} F^{o}$ & 3 & $5 p^{5} 9 g$ & ${ }^{3} H^{o}$ & 4 & 472.98 & -6.157 & $2.08 \times 10^{4}$ \\
\hline $5 p^{5} 5 d$ & ${ }^{3} P^{o}$ & 2 & $5 p^{5} 9 g$ & ${ }^{3} F^{o}$ & 4 & 417.71 & -5.456 & $1.34 \times 10^{5}$ \\
\hline $5 p^{5} 5 d$ & ${ }^{3} F^{o}$ & 4 & $5 p^{5} 9 g$ & ${ }^{3} F^{o}$ & 4 & 419.24 & -6.01 & $3.71 \times 10^{4}$ \\
\hline $5 p^{5} 5 d$ & ${ }^{3} D^{o}$ & 2 & $5 p^{5} 9 g$ & ${ }^{3} F^{o}$ & 4 & 467.59 & -5.518 & $9.25 \times 10^{4}$ \\
\hline $5 p^{5} 5 d$ & ${ }^{1} F^{o}$ & 3 & $5 p^{5} 9 g$ & ${ }^{3} F^{o}$ & 4 & 472.97 & -6.101 & $2.36 \times 10^{4}$ \\
\hline $5 p^{5} 6 s$ & ${ }^{3} P^{o}$ & 2 & $5 p^{5} 9 g$ & ${ }^{3} F^{o}$ & 4 & 490.37 & -5.453 & $9.77 \times 10^{4}$ \\
\hline $5 p^{5} 6 d$ & ${ }^{3} P^{o}$ & 2 & $5 p^{5} 9 g$ & ${ }^{3} F^{o}$ & 4 & 861.3 & -5.803 & $1.42 \times 10^{4}$ \\
\hline $5 p^{5} 5 d$ & ${ }^{3} F^{o}$ & 3 & $5 p^{5} 9 g$ & ${ }^{3} H^{o}$ & 5 & 421.42 & -5.522 & $1.13 \times 10^{5}$ \\
\hline $5 p^{5} 5 d$ & ${ }^{1} F^{o}$ & 3 & $5 p^{5} 9 g$ & ${ }^{3} H^{o}$ & 5 & 472.96 & -5.038 & $2.73 \times 10^{5}$ \\
\hline $5 p^{5} 6 d$ & ${ }^{3} F^{o}$ & 3 & $5 p^{5} 9 g$ & ${ }^{3} H^{o}$ & 5 & 1060.68 & -5.709 & $1.16 \times 10^{4}$ \\
\hline $5 p^{5} 5 d$ & ${ }^{3} P^{o}$ & 1 & $5 p^{5} 9 g$ & ${ }^{3} G^{o}$ & 3 & 410.01 & -5.815 & $6.07 \times 10^{4}$ \\
\hline $5 p^{5} 5 d$ & ${ }^{3} P^{o}$ & 2 & $5 p^{5} 9 g$ & ${ }^{3} G^{o}$ & 3 & 417.67 & -6.473 & $1.29 \times 10^{4}$ \\
\hline $5 p^{5} 5 d$ & ${ }^{3} F^{o}$ & 3 & $5 p^{5} 9 g$ & ${ }^{3} G^{o}$ & 3 & 421.39 & -6.363 & $1.63 \times 10^{4}$ \\
\hline $5 p^{5} 5 d$ & ${ }^{3} D^{o}$ & 1 & $5 p^{5} 9 g$ & ${ }^{3} G^{o}$ & 3 & 451.62 & -6.246 & $1.86 \times 10^{4}$ \\
\hline $5 p^{5} 6 s$ & ${ }^{3} P^{o}$ & 2 & $5 p^{5} 9 g$ & ${ }^{3} G^{o}$ & 3 & 490.31 & -6.408 & $1.09 \times 10^{4}$ \\
\hline $5 p^{5} 6 s$ & ${ }^{1} P^{o}$ & 1 & $5 p^{5} 9 g$ & ${ }^{3} G^{o}$ & 3 & 494.8 & -6.15 & $1.93 \times 10^{4}$ \\
\hline
\end{tabular}


TABLE VI (cont.)

\begin{tabular}{|c|c|c|c|c|c|c|c|c|}
\hline \multicolumn{3}{|c|}{ Lower Level } & \multicolumn{3}{|c|}{ Upper Level } & $\lambda[\AA]$ & $\log (g f)$ & $g A_{j i}\left[\mathrm{~s}^{-1}\right]$ \\
\hline Conf. & Term & $J$ & Conf. & Term & $J$ & HFR & HFR & HFR \\
\hline $5 p^{5} 5 d$ & ${ }^{1} P^{o}$ & 1 & $5 p^{5} 9 g$ & ${ }^{3} G^{o}$ & 3 & 536.68 & -4.118 & $1.77 \times 10^{6}$ \\
\hline $5 p^{5} 6 d$ & ${ }^{3} P^{o}$ & 1 & $5 p^{5} 9 g$ & ${ }^{3} G^{o}$ & 3 & 852.34 & -5.948 & $1.04 \times 10^{4}$ \\
\hline $5 p^{5} 6 d$ & ${ }^{1} P^{o}$ & 1 & $5 p^{5} 9 g$ & ${ }^{3} G^{o}$ & 3 & 896.61 & -5.177 & $5.52 \times 10^{4}$ \\
\hline $5 p^{5} 6 d$ & ${ }^{3} D^{o}$ & 1 & $5 p^{5} 9 g$ & ${ }^{3} G^{o}$ & 3 & 1082.3 & -5.009 & $5.57 \times 10^{4}$ \\
\hline $5 p^{5} 5 g$ & ${ }^{3} H^{o}$ & 4 & $5 p^{5} 9 i$ & ${ }^{3} K^{o}$ & 6 & 2005.65 & -4.769 & $2.82 \times 10^{4}$ \\
\hline $5 p^{5} 5 g$ & ${ }^{3} G^{o}$ & 3 & $5 p^{5} 9 i$ & ${ }^{3} I^{o}$ & 5 & 2005.77 & -4.915 & $2.02 \times 10^{4}$ \\
\hline $5 p^{5} 5 g$ & ${ }^{3} H^{o}$ & 5 & $5 p^{5} 9 i$ & ${ }^{3} K^{o}$ & 7 & 2006.91 & -4.691 & $3.37 \times 10^{4}$ \\
\hline $5 p^{5} 5 g$ & ${ }^{3} F^{o}$ & 4 & $5 p^{5} 9 i$ & ${ }^{3} H^{o}$ & 6 & 2003.3 & -4.812 & $2.56 \times 10^{4}$ \\
\hline $5 p^{5} 5 d$ & ${ }^{3} F^{o}$ & 3 & $5 p^{5} 10 g$ & ${ }^{3} H^{o}$ & 4 & 414.12 & -6.211 & $2.39 \times 10^{4}$ \\
\hline $5 p^{5} 5 d$ & ${ }^{3} F^{o}$ & 2 & $5 p^{5} 10 g$ & ${ }^{3} H^{o}$ & 4 & 451.64 & -5.391 & $1.33 \times 10^{5}$ \\
\hline $5 p^{5} 5 d$ & ${ }^{1} F^{o}$ & 3 & $5 p^{5} 10 g$ & ${ }^{3} H^{o}$ & 4 & 463.78 & -6.086 & $2.55 \times 10^{4}$ \\
\hline $5 p^{5} 5 d$ & ${ }^{3} F^{o}$ & 4 & $5 p^{5} 10 g$ & ${ }^{3} F^{o}$ & 4 & 412 & -4.725 & $7.40 \times 10^{5}$ \\
\hline $5 p^{5} 5 d$ & ${ }^{3} F^{o}$ & 3 & $5 p^{5} 10 g$ & ${ }^{3} F^{o}$ & 4 & 414.12 & -6.131 & $2.88 \times 10^{4}$ \\
\hline $5 p^{5} 5 d$ & ${ }^{3} D^{o}$ & 2 & $5 p^{5} 10 g$ & ${ }^{3} F^{o}$ & 4 & 458.61 & -5.397 & $1.27 \times 10^{5}$ \\
\hline $5 p^{5} 5 d$ & ${ }^{1} F^{o}$ & 3 & $5 p^{5} 10 g$ & ${ }^{3} F^{o}$ & 4 & 463.77 & -6.029 & $2.90 \times 10^{4}$ \\
\hline $5 p^{5} 5 d$ & ${ }^{3} F^{o}$ & 4 & $5 p^{5} 10 g$ & ${ }^{3} H^{o}$ & 5 & 411.99 & -5.759 & $6.84 \times 10^{4}$ \\
\hline $5 p^{5} 5 d$ & ${ }^{1} F^{o}$ & 3 & $5 p^{5} 10 g$ & ${ }^{3} H^{o}$ & 5 & 463.77 & -5.232 & $1.82 \times 10^{5}$ \\
\hline $5 p^{5} 6 d$ & ${ }^{3} F^{o}$ & 3 & $5 p^{5} 10 g$ & ${ }^{3} H^{o}$ & 5 & 1015.55 & -5.795 & $1.04 \times 10^{4}$ \\
\hline $5 p^{5} 5 d$ & ${ }^{3} F^{o}$ & 4 & $5 p^{5} 10 g$ & ${ }^{3} G^{o}$ & 3 & 411.97 & -5.767 & $6.72 \times 10^{4}$ \\
\hline $5 p^{5} 5 d$ & ${ }^{3} F^{o}$ & 3 & $5 p^{5} 10 g$ & ${ }^{3} G^{o}$ & 3 & 414.08 & -5.284 & $2.02 \times 10^{5}$ \\
\hline $5 p^{5} 5 d$ & ${ }^{3} D^{o}$ & 1 & $5 p^{5} 10 g$ & ${ }^{3} G^{o}$ & 3 & 443.24 & -6.065 & $2.92 \times 10^{4}$ \\
\hline $5 p^{5} 5 d$ & ${ }^{3} F^{o}$ & 2 & $5 p^{5} 10 g$ & ${ }^{3} G^{o}$ & 3 & 451.59 & -6.437 & $1.20 \times 10^{4}$ \\
\hline $5 p^{5} 5 d$ & ${ }^{3} D^{o}$ & 2 & $5 p^{5} 10 g$ & ${ }^{3} G^{o}$ & 3 & 458.57 & -6.421 & $1.20 \times 10^{4}$ \\
\hline $5 p^{5} 5 d$ & ${ }^{1} F^{o}$ & 3 & $5 p^{5} 10 g$ & ${ }^{3} G^{o}$ & 3 & 463.74 & -6.371 & $1.32 \times 10^{4}$ \\
\hline $5 p^{5} 5 d$ & ${ }^{1} P^{o}$ & 1 & $5 p^{5} 10 g$ & ${ }^{3} G^{o}$ & 3 & 524.89 & -5.378 & $1.01 \times 10^{5}$ \\
\hline $5 p^{5} 5 g$ & ${ }^{3} H^{o}$ & 4 & $5 p^{5} 10 i$ & ${ }^{3} K^{o}$ & 6 & 1852.49 & -4.957 & $2.14 \times 10^{4}$ \\
\hline $5 p^{5} 5 g$ & ${ }^{3} H^{o}$ & 5 & $5 p^{5} 10 i$ & ${ }^{3} K^{o}$ & 7 & 1853.59 & -4.888 & $2.51 \times 10^{4}$ \\
\hline $5 p^{5} 5 d$ & ${ }^{3} F^{o}$ & 4 & $5 p^{5} 10 i$ & ${ }^{3} H^{o}$ & 6 & 411.69 & -5.222 & $2.36 \times 10^{5}$ \\
\hline $5 p^{5} 5 d$ & ${ }^{3} F^{o}$ & 3 & $5 p^{5} 10 i$ & ${ }^{3} I^{o}$ & 5 & 413.81 & -5.741 & $7.08 \times 10^{4}$ \\
\hline $5 p^{5} 5 g$ & ${ }^{3} F^{o}$ & 4 & $5 p^{5} 10 i$ & ${ }^{3} H^{o}$ & 6 & 1850.51 & -4.99 & $2.00 \times 10^{4}$ \\
\hline $5 p^{5} 5 g$ & ${ }^{3} G^{o}$ & 3 & $5 p^{5} 10 i$ & ${ }^{3} I^{o}$ & 5 & 1852.6 & -5.105 & $1.53 \times 10^{4}$ \\
\hline
\end{tabular}


New magnetic dipole (M1) transitions for La IV.

TABLE VII

\begin{tabular}{|c|c|c|c|c|c|c|c|c|}
\hline \multicolumn{3}{|c|}{ Lower level } & \multicolumn{3}{|c|}{ Upper level } & $\lambda[\AA]$ & $\log (g f)$ & $g A_{j i}\left[\mathrm{~s}^{-1}\right]$ \\
\hline Conf. & Term & $J$ & Conf. & Term & $\mathrm{J}$ & HFR & HFR & HFR \\
\hline $5 p^{5} 7 h$ & ${ }^{3} I$ & 7 & $5 p^{5} 7 h$ & ${ }^{3} I$ & 6 & 4692.29 & -5.366 & $1.30 \times 10^{3}$ \\
\hline $5 p^{5} 8 h$ & ${ }^{3} I$ & 7 & $5 p^{5} 8 h$ & ${ }^{3} I$ & 6 & 4696.58 & -5.366 & $1.30 \times 10^{3}$ \\
\hline $5 p^{5} 9 h$ & ${ }^{3} I$ & 7 & $5 p^{5} 9 h$ & ${ }^{3} I$ & 6 & 4699.12 & -5.365 & $1.30 \times 10^{3}$ \\
\hline $5 p^{5} 10 h$ & ${ }^{3} I$ & 7 & $5 p^{5} 10 h$ & ${ }^{3} I$ & 6 & 4700.72 & -5.365 & $1.30 \times 10^{3}$ \\
\hline $5 p^{5} 6 h$ & ${ }^{3} I$ & 7 & $5 p^{5} 6 h$ & ${ }^{3} I$ & 6 & 4688.68 & -5.397 & $1.22 \times 10^{3}$ \\
\hline $5 p^{5} 7 h$ & ${ }^{1} I$ & 6 & $5 p^{5} 7 h$ & ${ }^{3} I$ & 5 & 4692.62 & -5.434 & $1.12 \times 10^{3}$ \\
\hline $5 p^{5} 9 h$ & ${ }^{1} I$ & 6 & $5 p^{5} 9 h$ & ${ }^{3} I$ & 5 & 4699.36 & -5.433 & $1.12 \times 10^{3}$ \\
\hline $5 p^{5} 8 h$ & ${ }^{1} I$ & 6 & $5 p^{5} 8 h$ & ${ }^{3} I$ & 5 & 4696.89 & -5.433 & $1.11 \times 10^{3}$ \\
\hline $5 p^{5} 10 h$ & ${ }^{1} I$ & 6 & $5 p^{5} 10 h$ & ${ }^{3} I$ & 5 & 4700.92 & -5.433 & $1.11 \times 10^{3}$ \\
\hline $5 p^{5} 6 h$ & ${ }^{1} I$ & 6 & $5 p^{5} 6 h$ & ${ }^{3} I$ & 5 & 4688.94 & -5.464 & $1.04 \times 10^{3}$ \\
\hline $5 p^{5} 5 f$ & ${ }^{3} G$ & 5 & $5 p^{5} 5 f$ & ${ }^{3} G$ & 4 & 4555.49 & -5.524 & $9.63 \times 10^{2}$ \\
\hline $5 p^{5} 8 f$ & ${ }^{3} G$ & 5 & $5 p^{5} 8 f$ & ${ }^{3} G$ & 4 & 4679.33 & -5.504 & $9.55 \times 10^{2}$ \\
\hline $5 p^{5} 9 f$ & ${ }^{3} G$ & 5 & $5 p^{5} 9 f$ & ${ }^{3} G$ & 4 & 4688.53 & -5.502 & $9.55 \times 10^{2}$ \\
\hline $5 p^{5} 10 f$ & ${ }^{3} G$ & 5 & $5 p^{5} 10 f$ & ${ }^{3} G$ & 4 & 4693.72 & -5.501 & $9.54 \times 10^{2}$ \\
\hline $5 p^{5} 7 f$ & ${ }^{3} G$ & 5 & $5 p^{5} 7 f$ & ${ }^{3} G$ & 4 & 4649.58 & -5.577 & $8.17 \times 10^{2}$ \\
\hline $5 p^{5} 10 f$ & ${ }^{3} G$ & 4 & $5 p^{5} 10 f$ & ${ }^{3} G$ & 3 & 4703.81 & -5.591 & $7.74 \times 10^{2}$ \\
\hline $5 p^{5} 8 f$ & ${ }^{3} G$ & 4 & $5 p^{5} 8 f$ & ${ }^{3} G$ & 3 & 4700.07 & -5.592 & $7.73 \times 10^{2}$ \\
\hline $5 p^{5} 8 h$ & ${ }^{3} G$ & 4 & $5 p^{5} 8 h$ & ${ }^{3} G$ & 5 & 4691.28 & -5.598 & $7.64 \times 10^{2}$ \\
\hline $5 p^{5} 5 f$ & ${ }^{1} G$ & 4 & $5 p^{5} 5 f$ & ${ }^{3} G$ & 3 & 4638.21 & -5.609 & $7.64 \times 10^{2}$ \\
\hline $5 p^{5} 7 h$ & ${ }^{3} G$ & 4 & $5 p^{5} 7 h$ & ${ }^{3} G$ & 5 & 4684.1 & -5.6 & $7.63 \times 10^{2}$ \\
\hline $5 p^{5} 9 h$ & ${ }^{3} G$ & 4 & $5 p^{5} 9 h$ & ${ }^{3} G$ & 5 & 4695.54 & -5.6 & $7.60 \times 10^{2}$ \\
\hline $5 p^{5} 10 h$ & ${ }^{3} G$ & 4 & $5 p^{5} 10 h$ & ${ }^{3} G$ & 5 & 4698.21 & -5.6 & $7.59 \times 10^{2}$ \\
\hline $5 p^{5} 9 f$ & ${ }^{3} G$ & 4 & $5 p^{5} 9 f$ & ${ }^{3} G$ & 3 & 4700.36 & -5.6 & $7.59 \times 10^{2}$ \\
\hline $5 p^{5} 6 p$ & ${ }^{3} D$ & 3 & $5 p^{5} 6 p$ & ${ }^{3} D$ & 2 & 4367.97 & -5.71 & $6.82 \times 10^{2}$ \\
\hline $5 p^{5} 9 h$ & ${ }^{3} G$ & 5 & $5 p^{5} 9 h$ & ${ }^{3} G$ & 5 & 4709.82 & -5.648 & $6.76 \times 10^{2}$ \\
\hline $5 p^{5} 7 h$ & ${ }^{3} G$ & 5 & $5 p^{5} 7 h$ & ${ }^{3} G$ & 5 & 4714.49 & -5.648 & $6.76 \times 10^{2}$ \\
\hline $5 p^{5} 10 h$ & ${ }^{3} H$ & 6 & $5 p^{5} 10 h$ & ${ }^{3} I$ & 6 & 4711.65 & -5.649 & $6.75 \times 10^{2}$ \\
\hline $5 p^{5} 10 h$ & ${ }^{3} G$ & 5 & $5 p^{5} 10 h$ & ${ }^{3} G$ & 5 & 4708.36 & -5.649 & $6.74 \times 10^{2}$ \\
\hline $5 p^{5} 9 h$ & ${ }^{3} H$ & 6 & $5 p^{5} 9 h$ & ${ }^{3} I$ & 6 & 4714.2 & -5.649 & $6.74 \times 10^{2}$ \\
\hline $5 p^{5} 7 h$ & ${ }^{3} H$ & 6 & $5 p^{5} 7 h$ & ${ }^{3} I$ & 6 & 4723.97 & -5.648 & $6.72 \times 10^{2}$ \\
\hline $5 p^{5} 4 f$ & ${ }^{3} G$ & 5 & $5 p^{5} 4 f$ & ${ }^{3} F$ & 4 & 4383.75 & -5.714 & $6.71 \times 10^{2}$ \\
\hline $5 p^{5} 6 h$ & ${ }^{3} G$ & 4 & $5 p^{5} 6 h$ & ${ }^{3} G$ & 5 & 4677.07 & -5.66 & $6.68 \times 10^{2}$ \\
\hline $5 p^{5} 8 h$ & ${ }^{3} H$ & 6 & $5 p^{5} 8 h$ & ${ }^{3} I$ & 6 & 4721.36 & -5.679 & $6.27 \times 10^{2}$ \\
\hline $5 p^{5} 6 h$ & ${ }^{3} H$ & 6 & $5 p^{5} 6 h$ & ${ }^{3} I$ & 6 & 4738.87 & -5.677 & $6.24 \times 10^{2}$ \\
\hline $5 p^{5} 7 h$ & ${ }^{3} G$ & 3 & $5 p^{5} 7 h$ & ${ }^{3} H$ & 4 & 4683.57 & -5.698 & $6.09 \times 10^{2}$ \\
\hline $5 p^{5} 8 h$ & ${ }^{3} G$ & 3 & $5 p^{5} 8 h$ & ${ }^{3} H$ & 4 & 4690.83 & -5.697 & $6.09 \times 10^{2}$ \\
\hline $5 p^{5} 9 h$ & ${ }^{3} G$ & 3 & $5 p^{5} 9 h$ & ${ }^{3} H$ & 4 & 4695.11 & -5.697 & $6.08 \times 10^{2}$ \\
\hline $5 p^{5} 10 h$ & ${ }^{3} G$ & 3 & $5 p^{5} 10 h$ & ${ }^{3} H$ & 4 & 4697.84 & -5.697 & $6.08 \times 10^{2}$ \\
\hline $5 p^{5} 10 p$ & ${ }^{3} D$ & 3 & $5 p^{5} 10 p$ & ${ }^{3} D$ & 2 & 4688.88 & -5.7 & $6.05 \times 10^{2}$ \\
\hline $5 p^{5} 8 p$ & ${ }^{3} D$ & 3 & $5 p^{5} 8 p$ & ${ }^{3} D$ & 2 & 4658.78 & -5.709 & $6.01 \times 10^{2}$ \\
\hline $5 p^{5} 6 h$ & ${ }^{3} G$ & 5 & $5 p^{5} 6 h$ & ${ }^{3} G$ & 5 & 4727.08 & -5.702 & $5.93 \times 10^{2}$ \\
\hline $5 p^{5} 8 h$ & ${ }^{3} G$ & 5 & $5 p^{5} 8 h$ & ${ }^{3} G$ & 5 & 4717.94 & -5.706 & $5.90 \times 10^{2}$ \\
\hline $5 p^{5} 9 p$ & ${ }^{3} D$ & 3 & $5 p^{5} 9 p$ & ${ }^{3} D$ & 2 & 4688.5 & -5.722 & $5.76 \times 10^{2}$ \\
\hline $5 p^{5} 7 p$ & ${ }^{3} D$ & 3 & $5 p^{5} 7 p$ & ${ }^{3} D$ & 2 & 4653.24 & -5.737 & $5.65 \times 10^{2}$ \\
\hline $5 p^{5} 9 h$ & ${ }^{1} H$ & 5 & $5 p^{5} 9 h$ & ${ }^{3} I$ & 5 & 4714.26 & -5.727 & $5.63 \times 10^{2}$ \\
\hline $5 p^{5} 7 h$ & ${ }^{1} H$ & 5 & $5 p^{5} 7 h$ & ${ }^{3} I$ & 5 & 4724.05 & -5.725 & $5.63 \times 10^{2}$ \\
\hline $5 p^{5} 10 h$ & ${ }^{1} H$ & 5 & $5 p^{5} 10 h$ & ${ }^{3} I$ & 5 & 4711.7 & -5.728 & $5.62 \times 10^{2}$ \\
\hline $5 p^{5} 7 h$ & ${ }^{3} H$ & 4 & $5 p^{5} 7 h$ & ${ }^{3} H$ & 4 & 4714.43 & -5.736 & $5.52 \times 10^{2}$ \\
\hline $5 p^{5} 9 h$ & ${ }^{3} H$ & 4 & $5 p^{5} 9 h$ & ${ }^{3} H$ & 4 & 4709.76 & -5.737 & $5.51 \times 10^{2}$ \\
\hline $5 p^{5} 10 h$ & ${ }^{3} H$ & 4 & $5 p^{5} 10 h$ & ${ }^{3} H$ & 4 & 4708.32 & -5.737 & $5.51 \times 10^{2}$ \\
\hline $5 p^{5} 6 h$ & ${ }^{3} G$ & 3 & $5 p^{5} 6 h$ & ${ }^{3} H$ & 4 & 4676.55 & -5.757 & $5.33 \times 10^{2}$ \\
\hline $5 p^{5} 7 f$ & ${ }^{3} G$ & 4 & $5 p^{5} 7 f$ & ${ }^{3} G$ & 3 & 4665.77 & -5.761 & $5.32 \times 10^{2}$ \\
\hline
\end{tabular}


TABLE VII (cont.)

\begin{tabular}{|c|c|c|c|c|c|c|c|c|}
\hline \multicolumn{3}{|c|}{ Lower level } & \multicolumn{3}{|c|}{ Upper level } & $\lambda[\AA]$ & $\log (g f)$ & $g A_{j i}\left[\mathrm{~s}^{-1}\right]$ \\
\hline Conf. & Term & $J$ & Conf. & Term & $\mathrm{J}$ & HFR & HFR & HFR \\
\hline $5 p^{5} 6 h$ & ${ }^{1} H$ & 5 & $5 p^{5} 6 h$ & ${ }^{3} I$ & 5 & 4738.96 & -5.75 & $5.28 \times 10^{2}$ \\
\hline $5 p^{5} 8 h$ & ${ }^{1} H$ & 5 & $5 p^{5} 8 h$ & ${ }^{3} I$ & 5 & 4721.45 & -5.756 & $5.25 \times 10^{2}$ \\
\hline $5 p^{5} 6 f$ & ${ }^{3} G$ & 5 & $5 p^{5} 6 f$ & ${ }^{3} G$ & 4 & 4566.59 & -5.802 & $5.05 \times 10^{2}$ \\
\hline $5 p^{5} 8 h$ & ${ }^{3} H$ & 4 & $5 p^{5} 8 h$ & ${ }^{3} H$ & 4 & 4717.73 & -5.792 & $4.84 \times 10^{2}$ \\
\hline $5 p^{5} 6 h$ & ${ }^{3} H$ & 4 & $5 p^{5} 6 h$ & ${ }^{3} H$ & 4 & 4726.89 & -5.791 & $4.83 \times 10^{2}$ \\
\hline $5 p^{5} 10 f$ & ${ }^{3} F$ & 4 & $5 p^{5} 10 f$ & ${ }^{3} G$ & 4 & 4729.02 & -5.825 & $4.47 \times 10^{2}$ \\
\hline $5 p^{5} 8 f$ & ${ }^{3} F$ & 4 & $5 p^{5} 8 f$ & ${ }^{3} G$ & 4 & 4754.3 & -5.823 & $4.43 \times 10^{2}$ \\
\hline $5 p^{5} 9 h$ & ${ }^{3} H$ & 6 & $5 p^{5} 9 h$ & ${ }^{3} G$ & 5 & 4714.04 & -5.835 & $4.39 \times 10^{2}$ \\
\hline $5 p^{5} 7 h$ & ${ }^{3} H$ & 6 & $5 p^{5} 7 h$ & ${ }^{3} G$ & 5 & 4723.45 & -5.833 & $4.39 \times 10^{2}$ \\
\hline $5 p^{5} 10 h$ & ${ }^{3} H$ & 6 & $5 p^{5} 10 h$ & ${ }^{3} G$ & 5 & 4711.54 & -5.836 & $4.39 \times 10^{2}$ \\
\hline $5 p^{5} 10 p$ & ${ }^{3} D$ & 2 & $5 p^{5} 10 p$ & ${ }^{3} D$ & 1 & 4714.09 & -5.848 & $4.26 \times 10^{2}$ \\
\hline $5 p^{5} 6 p$ & ${ }^{3} D$ & 2 & $5 p^{5} 6 p$ & ${ }^{3} D$ & 1 & 4741.86 & -5.847 & $4.22 \times 10^{2}$ \\
\hline $5 p^{5} 6 f$ & ${ }^{1} G$ & 4 & $5 p^{5} 6 f$ & ${ }^{3} G$ & 3 & 4756.99 & -5.845 & $4.21 \times 10^{2}$ \\
\hline $5 p^{5} 8 p$ & ${ }^{3} D$ & 2 & $5 p^{5} 8 p$ & ${ }^{3} D$ & 1 & 4725.04 & -5.855 & $4.17 \times 10^{2}$ \\
\hline $5 p^{5} 9 p$ & ${ }^{3} D$ & 2 & $5 p^{5} 9 p$ & ${ }^{3} D$ & 1 & 4722.14 & -5.857 & $4.16 \times 10^{2}$ \\
\hline $5 p^{5} 8 h$ & ${ }^{3} H$ & 6 & $5 p^{5} 8 h$ & ${ }^{3} G$ & 5 & 4721.11 & -5.864 & $4.10 \times 10^{2}$ \\
\hline $5 p^{5} 8 f$ & ${ }^{3} D$ & 3 & $5 p^{5} 8 f$ & ${ }^{3} D$ & 3 & 4720.2 & -5.864 & $4.09 \times 10^{2}$ \\
\hline $5 p^{5} 5 f$ & ${ }^{3} F$ & 4 & $5 p^{5} 5 f$ & ${ }^{3} G$ & 4 & 5030.48 & -5.809 & $4.09 \times 10^{2}$ \\
\hline $5 p^{5} 10 f$ & ${ }^{3} D$ & 3 & $5 p^{5} 10 f$ & ${ }^{3} D$ & 3 & 4715.15 & -5.867 & $4.07 \times 10^{2}$ \\
\hline $5 p^{5} 5 f$ & ${ }^{3} D$ & 3 & $5 p^{5} 5 f$ & ${ }^{3} D$ & 3 & 4756.61 & -5.866 & $4.01 \times 10^{2}$ \\
\hline $5 p^{5} 9 f$ & ${ }^{3} F$ & 4 & $5 p^{5} 9 f$ & ${ }^{3} G$ & 4 & 4725.57 & -5.878 & $3.96 \times 10^{2}$ \\
\hline $5 p^{5} 4 f$ & ${ }^{3} G$ & 4 & $5 p^{5} 4 f$ & ${ }^{3} G$ & 3 & 5256.94 & -5.788 & $3.93 \times 10^{2}$ \\
\hline $5 p^{5} 6 h$ & ${ }^{3} H$ & 6 & $5 p^{5} 6 h$ & ${ }^{3} G$ & 5 & 4741.11 & -5.885 & $3.87 \times 10^{2}$ \\
\hline $5 p^{5} 9 h$ & ${ }^{1} H$ & 5 & $5 p^{5} 9 h$ & ${ }^{3} H$ & 4 & 4713.8 & -5.912 & $3.67 \times 10^{2}$ \\
\hline $5 p^{5} 10 h$ & ${ }^{1} H$ & 5 & $5 p^{5} 10 h$ & ${ }^{3} H$ & 4 & 4711.34 & -5.913 & $3.67 \times 10^{2}$ \\
\hline $5 p^{5} 7 h$ & ${ }^{1} H$ & 5 & $5 p^{5} 7 h$ & ${ }^{3} H$ & 4 & 4723.16 & -5.911 & $3.67 \times 10^{2}$ \\
\hline $5 p^{5} 6 f$ & ${ }^{3} G$ & 5 & $5 p^{5} 7 f$ & ${ }^{3} F$ & 4 & 4695.05 & -5.924 & $3.61 \times 10^{2}$ \\
\hline $5 p^{5} 8 h$ & ${ }^{1} H$ & 5 & $5 p^{5} 8 h$ & ${ }^{3} H$ & 4 & 4720.85 & -5.942 & $3.42 \times 10^{2}$ \\
\hline $5 p^{5} 6 f$ & ${ }^{1} G$ & 4 & $5 p^{5} 7 f$ & ${ }^{1} F$ & 3 & 4596.23 & -5.966 & $3.42 \times 10^{2}$ \\
\hline $5 p^{5} 9 f$ & ${ }^{3} D$ & 3 & $5 p^{5} 9 f$ & ${ }^{3} D$ & 3 & 4690.41 & -5.949 & $3.41 \times 10^{2}$ \\
\hline $5 p^{5} 10 f$ & ${ }^{3} F$ & 4 & $5 p^{5} 10 f$ & ${ }^{3} D$ & 3 & 4731.68 & -5.965 & $3.23 \times 10^{2}$ \\
\hline $5 p^{5} 8 f$ & ${ }^{3} F$ & 4 & $5 p^{5} 8 f$ & ${ }^{3} D$ & 3 & 4757.86 & -5.963 & $3.21 \times 10^{2}$ \\
\hline $5 p^{5} 6 h$ & ${ }^{1} H$ & 5 & $5 p^{5} 6 h$ & ${ }^{3} H$ & 4 & 4740.75 & -5.967 & $3.20 \times 10^{2}$ \\
\hline $5 p^{5} 5 f$ & ${ }^{3} D$ & 2 & $5 p^{5} 5 f$ & ${ }^{3} D$ & 3 & 4458.37 & -6.03 & $3.13 \times 10^{2}$ \\
\hline $5 p^{5} 8 f$ & ${ }^{1} F$ & 3 & $5 p^{5} 8 f$ & ${ }^{3} G$ & 3 & 4759.43 & -5.983 & $3.06 \times 10^{2}$ \\
\hline $5 p^{5} 10 f$ & ${ }^{1} F$ & 3 & $5 p^{5} 10 f$ & ${ }^{3} G$ & 3 & 4731.62 & -5.991 & $3.04 \times 10^{2}$ \\
\hline $5 p^{5} 5 f$ & ${ }^{3} F$ & 4 & $5 p^{5} 5 f$ & ${ }^{3} D$ & 3 & 5021.03 & -5.951 & $2.96 \times 10^{2}$ \\
\hline $5 p^{5} 5 f$ & ${ }^{1} F$ & 3 & $5 p^{5} 5 f$ & ${ }^{3} G$ & 3 & 5054.54 & -5.956 & $2.89 \times 10^{2}$ \\
\hline $5 p^{5} 9 f$ & ${ }^{3} F$ & 4 & $5 p^{5} 9 f$ & ${ }^{3} D$ & 3 & 4728.83 & -6.029 & $2.79 \times 10^{2}$ \\
\hline $5 p^{5} 5 f$ & ${ }^{3} D$ & 1 & $5 p^{5} 5 f$ & ${ }^{3} F$ & 2 & 4319.52 & -6.117 & $2.73 \times 10^{2}$ \\
\hline $5 p^{5} 9 f$ & ${ }^{3} D$ & 1 & $5 p^{5} 9 f$ & ${ }^{3} F$ & 2 & 4655.08 & -6.072 & $2.61 \times 10^{2}$ \\
\hline $5 p^{5} 8 f$ & ${ }^{3} D$ & 1 & $5 p^{5} 8 f$ & ${ }^{3} F$ & 2 & 4635.45 & -6.076 & $2.60 \times 10^{2}$ \\
\hline $5 p^{5} 8 f$ & ${ }^{3} D$ & 2 & $5 p^{5} 8 f$ & ${ }^{3} D$ & 3 & 4675.65 & -6.072 & $2.59 \times 10^{2}$ \\
\hline $5 p^{5} 6 f$ & ${ }^{3} D$ & 3 & $5 p^{5} 6 f$ & ${ }^{3} D$ & 3 & 4660.79 & -6.075 & $2.59 \times 10^{2}$ \\
\hline $5 p^{5} 9 h$ & ${ }^{3} G$ & 5 & $5 p^{5} 9 h$ & ${ }^{3} I$ & 6 & 4709.99 & -6.071 & $2.55 \times 10^{2}$ \\
\hline $5 p^{5} 10 h$ & ${ }^{3} G$ & 5 & $5 p^{5} 10 h$ & ${ }^{3} I$ & 6 & 4708.46 & -6.071 & $2.55 \times 10^{2}$ \\
\hline $5 p^{5} 7 h$ & ${ }^{3} G$ & 5 & $5 p^{5} 7 h$ & ${ }^{3} I$ & 6 & 4715 & -6.07 & $2.55 \times 10^{2}$ \\
\hline $5 p^{5} 10 f$ & ${ }^{3} D$ & 1 & $5 p^{5} 10 f$ & ${ }^{3} F$ & 2 & 4678.08 & -6.078 & $2.55 \times 10^{2}$ \\
\hline $5 p^{5} 9 f$ & ${ }^{1} F$ & 3 & $5 p^{5} 9 f$ & ${ }^{3} G$ & 3 & 4720.18 & -6.088 & $2.45 \times 10^{2}$ \\
\hline $5 p^{5} 7 f$ & ${ }^{3} D$ & 1 & $5 p^{5} 7 f$ & ${ }^{3} F$ & 2 & 4567.69 & -6.124 & $2.40 \times 10^{2}$ \\
\hline $5 p^{5} 6 h$ & ${ }^{3} G$ & 5 & $5 p^{5} 6 h$ & ${ }^{3} I$ & 6 & 4724.85 & -6.1 & $2.37 \times 10^{2}$ \\
\hline $5 p^{5} 8 f$ & ${ }^{1} F$ & 3 & $5 p^{5} 8 f$ & ${ }^{3} F$ & 2 & 4730.84 & -6.103 & $2.35 \times 10^{2}$ \\
\hline
\end{tabular}


TABLE VII (cont.)

\begin{tabular}{|c|c|c|c|c|c|c|c|c|}
\hline \multicolumn{3}{|c|}{ Lower level } & \multicolumn{3}{|c|}{ Upper level } & $\lambda[\AA]$ & $\log (g f)$ & $g A_{j i}\left[\mathrm{~s}^{-1}\right]$ \\
\hline Conf. & Term & $J$ & Conf. & Term & $\mathrm{J}$ & HFR & HFR & HFR \\
\hline $5 p^{5} 10 f$ & ${ }^{1} F$ & 3 & $5 p^{5} 10 f$ & ${ }^{3} F$ & 2 & 4717.75 & -6.105 & $2.35 \times 10^{2}$ \\
\hline $5 p^{5} 6 f$ & ${ }^{3} F$ & 4 & $5 p^{5} 6 f$ & ${ }^{3} G$ & 4 & 4782 & -6.104 & $2.29 \times 10^{2}$ \\
\hline $5 p^{5} 5 f$ & ${ }^{1} F$ & 3 & $5 p^{5} 5 f$ & ${ }^{3} F$ & 2 & 4919.21 & -6.092 & $2.23 \times 10^{2}$ \\
\hline $5 p^{5} 8 h$ & ${ }^{3} G$ & 5 & $5 p^{5} 8 h$ & ${ }^{3} I$ & 6 & 4718.2 & -6.129 & $2.23 \times 10^{2}$ \\
\hline $5 p^{5} 5 f$ & ${ }^{1} D$ & 2 & $5 p^{5} 5 f$ & ${ }^{3} F$ & 2 & 4812.83 & -6.115 & $2.21 \times 10^{2}$ \\
\hline $5 p^{5} 9 f$ & ${ }^{3} D$ & 2 & $5 p^{5} 9 f$ & ${ }^{3} D$ & 3 & 4674.94 & -6.148 & $2.17 \times 10^{2}$ \\
\hline $5 p^{5} 10 f$ & ${ }^{3} D$ & 2 & $5 p^{5} 10 f$ & ${ }^{3} D$ & 3 & 4699.66 & -6.146 & $2.16 \times 10^{2}$ \\
\hline $5 p^{5} 7 f$ & ${ }^{3} D$ & 3 & $5 p^{5} 7 f$ & ${ }^{3} D$ & 3 & 4604.39 & -6.164 & $2.16 \times 10^{2}$ \\
\hline $5 p^{5} 7 f$ & ${ }^{3} F$ & 4 & $5 p^{5} 7 f$ & ${ }^{3} G$ & 4 & 4709.76 & -6.145 & $2.16 \times 10^{2}$ \\
\hline $5 p^{5} 4 f$ & ${ }^{3} D$ & 1 & $5 p^{5} 4 f$ & ${ }^{1} D$ & 2 & 3148.38 & -6.496 & $2.15 \times 10^{2}$ \\
\hline $5 p^{5} 10 p$ & ${ }^{3} P$ & 2 & $5 p^{5} 10 p$ & ${ }^{3} D$ & 2 & 4735.15 & -6.146 & $2.13 \times 10^{2}$ \\
\hline $5 p^{5} 10 p$ & ${ }^{3} P$ & 2 & $5 p^{5} 10 p$ & ${ }^{3} P$ & 1 & 4742.86 & -6.145 & $2.13 \times 10^{2}$ \\
\hline $5 p^{5} 9 f$ & ${ }^{1} D$ & 2 & $5 p^{5} 9 f$ & ${ }^{3} D$ & 3 & 4732.93 & -6.148 & $2.12 \times 10^{2}$ \\
\hline $5 p^{5} 9 p$ & ${ }^{3} P$ & 2 & $5 p^{5} 9 p$ & ${ }^{3} P$ & 1 & 4762.36 & -6.143 & $2.11 \times 10^{2}$ \\
\hline $5 p^{5} 7 h$ & ${ }^{3} H$ & 4 & $5 p^{5} 7 h$ & ${ }^{3} I$ & 5 & 4715.31 & -6.156 & $2.09 \times 10^{2}$ \\
\hline $5 p^{5} 9 h$ & ${ }^{3} H$ & 4 & $5 p^{5} 9 h$ & ${ }^{3} I$ & 5 & 4710.22 & -6.158 & $2.09 \times 10^{2}$ \\
\hline $5 p^{5} 10 h$ & ${ }^{3} H$ & 4 & $5 p^{5} 10 h$ & ${ }^{3} I$ & 5 & 4708.68 & -6.16 & $2.08 \times 10^{2}$ \\
\hline $5 p^{5} 8 p$ & ${ }^{3} P$ & 2 & $5 p^{5} 8 p$ & ${ }^{3} D$ & 2 & 4789.18 & -6.147 & $2.07 \times 10^{2}$ \\
\hline $5 p^{5} 8 p$ & ${ }^{3} P$ & 2 & $5 p^{5} 8 p$ & ${ }^{3} P$ & 1 & 4795.79 & -6.148 & $2.06 \times 10^{2}$ \\
\hline $5 p^{5} 6 p$ & ${ }^{3} P$ & 2 & $5 p^{5} 6 p$ & ${ }^{3} D$ & 2 & 5028 & -6.114 & $2.03 \times 10^{2}$ \\
\hline $5 p^{5} 9 p$ & ${ }^{3} P$ & 2 & $5 p^{5} 9 p$ & ${ }^{3} D$ & 2 & 4757.56 & -6.163 & $2.03 \times 10^{2}$ \\
\hline $5 p^{5} 7 p$ & ${ }^{3} P$ & 2 & $5 p^{5} 7 p$ & ${ }^{3} P$ & 1 & 4932.17 & -6.136 & $2.00 \times 10^{2}$ \\
\hline $5 p^{5} 10 f$ & ${ }^{1} D$ & 2 & $5 p^{5} 10 f$ & ${ }^{3} D$ & 3 & 4729.3 & -6.175 & $1.99 \times 10^{2}$ \\
\hline $5 p^{5} 6 h$ & ${ }^{3} H$ & 4 & $5 p^{5} 6 h$ & ${ }^{3} I$ & 5 & 4725.11 & -6.177 & $1.99 \times 10^{2}$ \\
\hline $5 p^{5} 4 f$ & ${ }^{3} F$ & 4 & $5 p^{5} 4 f$ & ${ }^{3} F$ & 4 & 6871.67 & -5.853 & $1.98 \times 10^{2}$ \\
\hline $5 p^{5} 6 f$ & ${ }^{3} F$ & 4 & $5 p^{5} 6 f$ & ${ }^{3} D$ & 3 & 4765.98 & -6.18 & $1.94 \times 10^{2}$ \\
\hline $5 p^{5} 8 f$ & ${ }^{1} D$ & 2 & $5 p^{5} 8 f$ & ${ }^{3} F$ & 2 & 4730.75 & -6.191 & $1.92 \times 10^{2}$ \\
\hline $5 p^{5} 7 p$ & ${ }^{3} P$ & 2 & $5 p^{5} 7 p$ & ${ }^{3} D$ & 2 & 4938.31 & -6.159 & $1.90 \times 10^{2}$ \\
\hline $5 p^{5} 9 f$ & ${ }^{1} F$ & 3 & $5 p^{5} 9 f$ & ${ }^{3} F$ & 2 & 4700.95 & -6.202 & $1.89 \times 10^{2}$ \\
\hline $5 p^{5} 7 f$ & ${ }^{1} D$ & 2 & $5 p^{5} 7 f$ & ${ }^{3} D$ & 3 & 4718.42 & -6.207 & $1.86 \times 10^{2}$ \\
\hline $5 p^{5} 6 f$ & ${ }^{3} D$ & 1 & $5 p^{5} 6 f$ & ${ }^{3} F$ & 2 & 4498.7 & -6.253 & $1.84 \times 10^{2}$ \\
\hline $5 p^{5} 8 h$ & ${ }^{3} H$ & 4 & $5 p^{5} 8 h$ & ${ }^{3} I$ & 5 & 4718.33 & -6.212 & $1.84 \times 10^{2}$ \\
\hline $5 p^{5} 10 p$ & ${ }^{1} P$ & 1 & $5 p^{5} 10 p$ & ${ }^{3} P$ & 1 & 4717.59 & -6.232 & $1.76 \times 10^{2}$ \\
\hline $5 p^{5} 10 f$ & ${ }^{1} D$ & 2 & $5 p^{5} 10 f$ & ${ }^{3} F$ & 2 & 4719.81 & -6.243 & $1.71 \times 10^{2}$ \\
\hline $5 p^{5} 6 f$ & ${ }^{1} F$ & 3 & $5 p^{5} 6 f$ & ${ }^{3} F$ & 2 & 4746.18 & -6.242 & $1.70 \times 10^{2}$ \\
\hline $5 p^{5} 8 f$ & ${ }^{1} D$ & 2 & $5 p^{5} 8 f$ & ${ }^{3} D$ & 3 & 4748.32 & -6.244 & $1.69 \times 10^{2}$ \\
\hline $5 p^{5} 6 f$ & ${ }^{1} F$ & 3 & $5 p^{5} 6 f$ & ${ }^{3} G$ & 3 & 4947.51 & -6.211 & $1.68 \times 10^{2}$ \\
\hline $5 p^{5} 8 p$ & ${ }^{1} P$ & 1 & $5 p^{5} 8 p$ & ${ }^{3} P$ & 1 & 4726.88 & -6.258 & $1.65 \times 10^{2}$ \\
\hline $5 p^{5} 6 f$ & ${ }^{3} G$ & 4 & $5 p^{5} 7 f$ & ${ }^{3} G$ & 4 & 4846.52 & -6.24 & $1.63 \times 10^{2}$ \\
\hline $5 p^{5} 10 f$ & ${ }^{3} D$ & 2 & $5 p^{5} 10 f$ & ${ }^{3} F$ & 2 & 4690.29 & -6.27 & $1.63 \times 10^{2}$ \\
\hline $5 p^{5} 9 p$ & ${ }^{1} P$ & 1 & $5 p^{5} 9 p$ & ${ }^{3} P$ & 1 & 4716.78 & -6.271 & $1.61 \times 10^{2}$ \\
\hline $5 p^{5} 7 f$ & ${ }^{3} D$ & 2 & $5 p^{5} 7 f$ & ${ }^{3} D$ & 3 & 4587.07 & -6.3 & $1.59 \times 10^{2}$ \\
\hline $5 p^{5} 6 f$ & ${ }^{3} F$ & 4 & $5 p^{5} 7 f$ & ${ }^{3} F$ & 4 & 4923.06 & -6.241 & $1.58 \times 10^{2}$ \\
\hline $5 p^{5} 6 f$ & ${ }^{3} D$ & 2 & $5 p^{5} 6 f$ & ${ }^{3} D$ & 3 & 4557.64 & -6.309 & $1.58 \times 10^{2}$ \\
\hline $5 p^{5} 9 f$ & ${ }^{3} D$ & 2 & $5 p^{5} 9 f$ & ${ }^{3} F$ & 2 & 4662.44 & -6.294 & $1.56 \times 10^{2}$ \\
\hline $5 p^{5} 6 f$ & ${ }^{3} D$ & 3 & $5 p^{5} 7 f$ & ${ }^{3} D$ & 3 & 4916.68 & -6.249 & $1.56 \times 10^{2}$ \\
\hline $5 p^{5} 7 p$ & ${ }^{1} P$ & 1 & $5 p^{5} 7 p$ & ${ }^{3} P$ & 1 & 4784.61 & -6.279 & $1.53 \times 10^{2}$ \\
\hline $5 p^{5} 9 f$ & ${ }^{1} D$ & 2 & $5 p^{5} 9 f$ & ${ }^{3} F$ & 2 & 4720.12 & -6.291 & $1.53 \times 10^{2}$ \\
\hline $5 p^{5} 7 f$ & ${ }^{3} G$ & 4 & $5 p^{5} 9 f$ & ${ }^{1} F$ & 3 & 4761.27 & -6.286 & $1.52 \times 10^{2}$ \\
\hline $5 p^{5} 6 f$ & ${ }^{1} D$ & 2 & $5 p^{5} 6 f$ & ${ }^{3} F$ & 2 & 4723.95 & -6.315 & $1.45 \times 10^{2}$ \\
\hline $5 p^{5} 7 p$ & ${ }^{3} S$ & 1 & $5 p^{5} 7 p$ & ${ }^{3} P$ & 0 & 4204.32 & -6.417 & $1.45 \times 10^{2}$ \\
\hline $5 p^{5} 6 f$ & ${ }^{1} F$ & 3 & $5 p^{5} 7 f$ & ${ }^{1} F$ & 3 & 4773.86 & -6.308 & $1.44 \times 10^{2}$ \\
\hline
\end{tabular}


TABLE VII (cont.)

\begin{tabular}{|c|c|c|c|c|c|c|c|c|}
\hline \multicolumn{3}{|c|}{ Lower level } & \multicolumn{3}{|c|}{ Upper level } & $\lambda[\AA]$ & $\log (g f)$ & $g A_{j i}\left[\mathrm{~s}^{-1}\right]$ \\
\hline Conf. & Term & $J$ & Conf. & Term & $\mathrm{J}$ & HFR & HFR & HFR \\
\hline $5 p^{5} 10 p$ & ${ }^{3} S$ & 1 & $5 p^{5} 10 p$ & ${ }^{3} P$ & 0 & 4607.93 & -6.34 & $1.43 \times 10^{2}$ \\
\hline $5 p^{5} 8 f$ & ${ }^{3} D$ & 2 & $5 p^{5} 8 f$ & ${ }^{3} F$ & 2 & 4658.62 & -6.334 & $1.43 \times 10^{2}$ \\
\hline $5 p^{5} 4 f$ & ${ }^{3} G$ & 3 & $5 p^{5} 4 f$ & ${ }^{3} G$ & 3 & 6692.95 & -6.023 & $1.41 \times 10^{2}$ \\
\hline $5 p^{5} 7 f$ & ${ }^{3} D$ & 2 & $5 p^{5} 7 f$ & ${ }^{3} F$ & 2 & 4576.94 & -6.357 & $1.40 \times 10^{2}$ \\
\hline $5 p^{5} 8 p$ & ${ }^{3} S$ & 1 & $5 p^{5} 8 p$ & ${ }^{3} P$ & 0 & 4434.11 & -6.393 & $1.37 \times 10^{2}$ \\
\hline $5 p^{5} 9 p$ & ${ }^{3} S$ & 1 & $5 p^{5} 9 p$ & ${ }^{3} P$ & 0 & 4536.18 & -6.379 & $1.35 \times 10^{2}$ \\
\hline $5 p^{5} 7 f$ & ${ }^{1} F$ & 3 & $5 p^{5} 7 f$ & ${ }^{3} G$ & 3 & 4846.86 & -6.323 & $1.35 \times 10^{2}$ \\
\hline $5 p^{5} 6 f$ & ${ }^{3} D$ & 3 & $5 p^{5} 7 f$ & ${ }^{3} D$ & 3 & 4853.96 & -6.327 & $1.33 \times 10^{2}$ \\
\hline $5 p^{5} 10 f$ & ${ }^{3} D$ & 3 & $5 p^{5} 10 f$ & ${ }^{3} G$ & 4 & 4712.51 & -6.356 & $1.32 \times 10^{2}$ \\
\hline $5 p^{5} 8 f$ & ${ }^{3} D$ & 3 & $5 p^{5} 8 f$ & ${ }^{3} G$ & 4 & 4716.7 & -6.356 & $1.32 \times 10^{2}$ \\
\hline $5 p^{5} 5 f$ & ${ }^{3} D$ & 3 & $5 p^{5} 5 f$ & ${ }^{3} G$ & 4 & 4765.1 & -6.353 & $1.30 \times 10^{2}$ \\
\hline $5 p^{5} 4 f$ & ${ }^{3} G$ & 3 & $5 p^{5} 4 f$ & ${ }^{1} D$ & 2 & 4605.27 & -6.388 & $1.29 \times 10^{2}$ \\
\hline $5 p^{5} 7 f$ & ${ }^{3} F$ & 4 & $5 p^{5} 7 f$ & ${ }^{3} D$ & 3 & 4701.19 & -6.372 & $1.28 \times 10^{2}$ \\
\hline $5 p^{5} 4 f$ & ${ }^{3} F$ & 4 & $5 p^{5} 4 f$ & ${ }^{3} F$ & 3 & 7068.46 & -6.037 & $1.23 \times 10^{2}$ \\
\hline $5 p^{5} 7 f$ & ${ }^{1} F$ & 3 & $5 p^{5} 7 f$ & ${ }^{3} F$ & 2 & 4809.89 & -6.377 & $1.21 \times 10^{2}$ \\
\hline $5 p^{5} 6 f$ & ${ }^{3} F$ & 4 & $5 p^{5} 7 f$ & ${ }^{3} D$ & 3 & 5033.88 & -6.342 & $1.20 \times 10^{2}$ \\
\hline $5 p^{5} 6 f$ & ${ }^{3} G$ & 4 & $5 p^{5} 7 f$ & ${ }^{3} D$ & 3 & 4837.45 & -6.378 & $1.19 \times 10^{2}$ \\
\hline $5 p^{5} 7 f$ & ${ }^{3} G$ & 5 & $5 p^{5} 9 f$ & ${ }^{3} F$ & 4 & 4745.21 & -6.407 & $1.16 \times 10^{2}$ \\
\hline $5 p^{5} 6 f$ & ${ }^{3} G$ & 3 & $5 p^{5} 7 f$ & ${ }^{3} G$ & 3 & 4680.08 & -6.42 & $1.16 \times 10^{2}$ \\
\hline $5 p^{5} 5 f$ & ${ }^{1} D$ & 2 & $5 p^{5} 5 f$ & ${ }^{3} D$ & 3 & 4858.39 & -6.401 & $1.12 \times 10^{2}$ \\
\hline $5 p^{5} 9 f$ & ${ }^{3} D$ & 3 & $5 p^{5} 9 f$ & ${ }^{3} G$ & 4 & 4687.2 & -6.438 & $1.11 \times 10^{2}$ \\
\hline $5 p^{5} 4 f$ & ${ }^{3} D$ & 2 & $5 p^{5} 4 f$ & ${ }^{3} F$ & 3 & 3956.15 & -6.588 & $1.10 \times 10^{2}$ \\
\hline $5 p^{5} 5 f$ & ${ }^{3} D$ & 2 & $5 p^{5} 5 f$ & ${ }^{3} F$ & 2 & 4419.98 & -6.501 & $1.08 \times 10^{2}$ \\
\hline $5 p^{5} 6 f$ & ${ }^{3} G$ & 3 & $5 p^{5} 7 f$ & ${ }^{3} F$ & 2 & 4645.6 & -6.483 & $1.02 \times 10^{2}$ \\
\hline $5 p^{5} 6 f$ & ${ }^{3} D$ & 2 & $5 p^{5} 7 f$ & ${ }^{3} D$ & 3 & 4802.03 & -6.461 & $1.00 \times 10^{2}$ \\
\hline $5 p^{5} 5 d$ & ${ }^{3} F^{o}$ & 3 & $5 p^{5} 5 d$ & ${ }^{3} F^{o}$ & 2 & 4985.55 & -5.784 & $4.41 \times 10^{2}$ \\
\hline $5 p^{5} 5 d$ & ${ }^{3} F^{o}$ & 2 & $5 p^{5} 5 d$ & ${ }^{3} F^{o}$ & 2 & 5946.85 & -6.153 & $1.33 \times 10^{2}$ \\
\hline $5 p^{5} 6 d$ & ${ }^{3} P^{o}$ & 2 & $5 p^{5} 6 d$ & ${ }^{3} P^{o}$ & 2 & 4640.78 & -6.062 & $2.69 \times 10^{2}$ \\
\hline $5 p^{5} 6 d$ & ${ }^{3} D^{o}$ & 3 & $5 p^{5} 6 d$ & ${ }^{3} P^{o}$ & 2 & 5035.97 & -6.025 & $2.49 \times 10^{2}$ \\
\hline $5 p^{5} 6 d$ & ${ }^{1} P^{o}$ & 1 & $5 p^{5} 6 d$ & ${ }^{3} P^{o}$ & 2 & 5899.23 & -6.116 & $1.47 \times 10^{2}$ \\
\hline $5 p^{5} 6 d$ & ${ }^{3} F^{o}$ & 4 & $5 p^{5} 6 d$ & ${ }^{3} F^{o}$ & 3 & 4513.22 & -5.609 & $8.05 \times 10^{2}$ \\
\hline $5 p^{5} 6 d$ & ${ }^{3} D^{o}$ & 3 & $5 p^{5} 6 d$ & ${ }^{3} F^{o}$ & 3 & 4965.66 & -5.933 & $3.15 \times 10^{2}$ \\
\hline $5 p^{5} 6 d$ & ${ }^{3} P^{o}$ & 1 & $5 p^{5} 6 d$ & ${ }^{3} D^{o}$ & 1 & 4011.57 & -6.34 & $1.89 \times 10^{2}$ \\
\hline $5 p^{5} 6 d$ & ${ }^{1} D^{o}$ & 2 & $5 p^{5} 6 d$ & ${ }^{3} D^{o}$ & 1 & 4440.69 & -6.308 & $1.66 \times 10^{2}$ \\
\hline $5 p^{5} 7 d$ & ${ }^{3} F^{o}$ & 3 & $5 p^{5} 7 d$ & ${ }^{3} F^{o}$ & 2 & 4762.4 & -5.759 & $5.12 \times 10^{2}$ \\
\hline $5 p^{5} 7 d$ & ${ }^{1} D^{o}$ & 2 & $5 p^{5} 7 d$ & ${ }^{3} F^{o}$ & 2 & 4913.68 & -6.362 & $1.20 \times 10^{2}$ \\
\hline $5 p^{5} 7 d$ & ${ }^{3} D^{o}$ & 3 & $5 p^{5} 7 d$ & ${ }^{3} P^{o}$ & 2 & 4918.15 & -6.332 & $1.29 \times 10^{2}$ \\
\hline $5 p^{5} 7 d$ & ${ }^{1} P^{o}$ & 1 & $5 p^{5} 7 d$ & ${ }^{3} P^{o}$ & 2 & 5461.62 & -6.177 & $1.49 \times 10^{2}$ \\
\hline $5 p^{5} 7 d$ & ${ }^{3} F^{o}$ & 4 & $5 p^{5} 7 d$ & ${ }^{3} F^{o}$ & 3 & 4665.07 & -5.675 & $6.48 \times 10^{2}$ \\
\hline $5 p^{5} 7 d$ & ${ }^{3} D^{o}$ & 3 & $5 p^{5} 7 d$ & ${ }^{3} F^{o}$ & 3 & 4876.13 & -6.293 & $1.43 \times 10^{2}$ \\
\hline $5 p^{5} 5 g$ & ${ }^{3} G^{o}$ & 3 & $5 p^{5} 7 d$ & ${ }^{3} F^{o}$ & 3 & 4916.8 & -6.368 & $1.18 \times 10^{2}$ \\
\hline $5 p^{5} 5 g$ & ${ }^{3} F^{o}$ & 3 & $5 p^{5} 5 g$ & ${ }^{3} F^{o}$ & 4 & 4638.75 & -5.845 & $4.43 \times 10^{2}$ \\
\hline $5 p^{5} 5 g$ & ${ }^{3} F^{o}$ & 4 & $5 p^{5} 5 g$ & ${ }^{3} F^{o}$ & 4 & 4787.65 & -5.803 & $4.58 \times 10^{2}$ \\
\hline $5 p^{5} 5 g$ & ${ }^{3} G^{o}$ & 5 & $5 p^{5} 5 g$ & ${ }^{3} F^{o}$ & 4 & 4849.37 & -5.951 & $3.18 \times 10^{2}$ \\
\hline $5 p^{5} 5 g$ & ${ }^{1} H^{o}$ & 5 & $5 p^{5} 5 g$ & ${ }^{3} H^{o}$ & 4 & 4669.59 & -5.538 & $8.86 \times 10^{2}$ \\
\hline $5 p^{5} 5 g$ & ${ }^{1} G^{o}$ & 4 & $5 p^{5} 5 g$ & ${ }^{3} H^{o}$ & 4 & 4832.59 & -5.842 & $4.11 \times 10^{2}$ \\
\hline $5 p^{5} 7 d$ & ${ }^{3} P^{o}$ & 2 & $5 p^{5} 5 g$ & ${ }^{3} G^{o}$ & 3 & 4603.48 & -6.324 & $1.49 \times 10^{2}$ \\
\hline $5 p^{5} 5 g$ & ${ }^{3} F^{o}$ & 2 & $5 p^{5} 5 g$ & ${ }^{3} G^{o}$ & 3 & 4610.3 & -6.182 & $2.06 \times 10^{2}$ \\
\hline $5 p^{5} 7 d$ & ${ }^{3} D^{o}$ & 3 & $5 p^{5} 5 g$ & ${ }^{3} G^{o}$ & 3 & 4766.51 & -6.322 & $1.40 \times 10^{2}$ \\
\hline $5 p^{5} 5 g$ & ${ }^{3} G^{o}$ & 3 & $5 p^{5} 5 g$ & ${ }^{3} G^{o}$ & 3 & 4805.37 & -6.194 & $1.85 \times 10^{2}$ \\
\hline $5 p^{5} 5 g$ & ${ }^{1} G^{o}$ & 4 & $5 p^{5} 5 g$ & ${ }^{3} G^{o}$ & 3 & 4831.81 & -6.063 & $2.47 \times 10^{2}$ \\
\hline $5 p^{5} 5 g$ & ${ }^{3} H^{o}$ & 6 & $5 p^{5} 5 g$ & ${ }^{3} H^{o}$ & 5 & 4654.89 & -5.461 & $1.07 \times 10^{3}$ \\
\hline
\end{tabular}


TABLE VII (cont.)

\begin{tabular}{|c|c|c|c|c|c|c|c|c|}
\hline \multicolumn{3}{|c|}{ Lower level } & \multicolumn{3}{|c|}{ Upper level } & $\lambda[\AA]$ & $\log (g f)$ & $g A_{j i}\left[\mathrm{~s}^{-1}\right]$ \\
\hline Conf. & Term & $J$ & Conf. & Term & $\mathrm{J}$ & HFR & HFR & HFR \\
\hline $5 p^{5} 5 g$ & ${ }^{3} F^{o}$ & 4 & $5 p^{5} 5 g$ & ${ }^{3} H^{o}$ & 5 & 4767.23 & -6.215 & $1.79 \times 10^{2}$ \\
\hline $5 p^{5} 5 g$ & ${ }^{3} G^{o}$ & 5 & $5 p^{5} 5 g$ & ${ }^{3} H^{o}$ & 5 & 4828.43 & -5.75 & $5.09 \times 10^{2}$ \\
\hline $5 p^{5} 8 s$ & ${ }^{1} P^{o}$ & 1 & $5 p^{5} 8 s$ & ${ }^{3} P^{o}$ & 0 & 4783.92 & -6.26 & $1.60 \times 10^{2}$ \\
\hline $5 p^{5} 8 s$ & ${ }^{3} P^{o}$ & 2 & $5 p^{5} 8 s$ & ${ }^{3} P^{o}$ & 1 & 4699.39 & -5.922 & $3.62 \times 10^{2}$ \\
\hline $5 p^{5} 6 g$ & ${ }^{3} F^{o}$ & 3 & $5 p^{5} 8 d$ & ${ }^{3} F^{o}$ & 2 & 4697.23 & -6.337 & $1.39 \times 10^{2}$ \\
\hline $5 p^{5} 8 d$ & ${ }^{3} F^{o}$ & 3 & $5 p^{5} 8 d$ & ${ }^{3} F^{o}$ & 2 & 4760.66 & -5.981 & $3.07 \times 10^{2}$ \\
\hline $5 p^{5} 6 g$ & ${ }^{3} F^{o}$ & 3 & $5 p^{5} 6 g$ & ${ }^{3} F^{o}$ & 4 & 4681.74 & -5.915 & $3.70 \times 10^{2}$ \\
\hline $5 p^{5} 6 g$ & ${ }^{3} F^{o}$ & 4 & $5 p^{5} 6 g$ & ${ }^{3} F^{o}$ & 4 & 4779.45 & -5.836 & $4.26 \times 10^{2}$ \\
\hline $5 p^{5} 6 g$ & ${ }^{3} G^{o}$ & 5 & $5 p^{5} 6 g$ & ${ }^{3} F^{o}$ & 4 & 4797.15 & -5.953 & $3.23 \times 10^{2}$ \\
\hline $5 p^{5} 6 g$ & ${ }^{1} H^{o}$ & 5 & $5 p^{5} 6 g$ & ${ }^{3} H^{o}$ & 4 & 4685.13 & -5.531 & $8.95 \times 10^{2}$ \\
\hline $5 p^{5} 6 g$ & ${ }^{3} G^{o}$ & 3 & $5 p^{5} 6 g$ & ${ }^{3} H^{o}$ & 4 & 4784.55 & -6.418 & $1.11 \times 10^{2}$ \\
\hline $5 p^{5} 6 g$ & ${ }^{1} G^{o}$ & 4 & $5 p^{5} 6 g$ & ${ }^{3} H^{o}$ & 4 & 4792.83 & -5.866 & $3.95 \times 10^{2}$ \\
\hline $5 p^{5} 6 g$ & ${ }^{3} H^{o}$ & 6 & $5 p^{5} 6 g$ & ${ }^{3} H^{o}$ & 5 & 4674.31 & -5.449 & $1.09 \times 10^{3}$ \\
\hline $5 p^{5} 6 g$ & ${ }^{3} F^{o}$ & 4 & $5 p^{5} 6 g$ & ${ }^{3} H^{o}$ & 5 & 4771.19 & -6.266 & $1.59 \times 10^{2}$ \\
\hline $5 p^{5} 6 g$ & ${ }^{3} G^{o}$ & 5 & $5 p^{5} 6 g$ & ${ }^{3} H^{o}$ & 5 & 4788.83 & -5.773 & $4.91 \times 10^{2}$ \\
\hline $5 p^{5} 6 g$ & ${ }^{3} F^{o}$ & 2 & $5 p^{5} 6 g$ & ${ }^{3} G^{o}$ & 3 & 4653.36 & -5.927 & $3.65 \times 10^{2}$ \\
\hline $5 p^{5} 6 g$ & ${ }^{3} G^{o}$ & 3 & $5 p^{5} 6 g$ & ${ }^{3} G^{o}$ & 3 & 4775.55 & -6.069 & $2.49 \times 10^{2}$ \\
\hline $5 p^{5} 6 g$ & ${ }^{1} G^{o}$ & 4 & $5 p^{5} 6 g$ & ${ }^{3} G^{o}$ & 3 & 4783.8 & -6.068 & $2.49 \times 10^{2}$ \\
\hline $5 p^{5} 8 d$ & ${ }^{3} D^{o}$ & 2 & $5 p^{5} 8 d$ & ${ }^{3} P^{o}$ & 2 & 4760.46 & -6.368 & $1.26 \times 10^{2}$ \\
\hline $5 p^{5} 8 d$ & ${ }^{1} D^{o}$ & 2 & $5 p^{5} 8 d$ & ${ }^{3} P^{o}$ & 2 & 4791.87 & -6.332 & $1.35 \times 10^{2}$ \\
\hline $5 p^{5} 8 d$ & ${ }^{3} D^{o}$ & 3 & $5 p^{5} 8 d$ & ${ }^{3} P^{o}$ & 2 & 4824.84 & -6.106 & $2.24 \times 10^{2}$ \\
\hline $5 p^{5} 8 d$ & ${ }^{1} P^{o}$ & 1 & $5 p^{5} 8 d$ & ${ }^{3} P^{o}$ & 2 & 5211.01 & -6.247 & $1.39 \times 10^{2}$ \\
\hline $5 p^{5} 8 d$ & ${ }^{3} F^{o}$ & 4 & $5 p^{5} 8 d$ & ${ }^{3} F^{o}$ & 3 & 4681.09 & -5.728 & $5.69 \times 10^{2}$ \\
\hline $5 p^{5} 8 d$ & ${ }^{3} D^{o}$ & 3 & $5 p^{5} 8 d$ & ${ }^{3} F^{o}$ & 3 & 4806.51 & -6.094 & $2.33 \times 10^{2}$ \\
\hline $5 p^{5} 9 s$ & ${ }^{1} P^{o}$ & 1 & $5 p^{5} 9 s$ & ${ }^{3} P^{o}$ & 0 & 4765.96 & -6.317 & $1.42 \times 10^{2}$ \\
\hline $5 p^{5} 9 s$ & ${ }^{3} P^{o}$ & 2 & $5 p^{5} 9 s$ & ${ }^{3} P^{o}$ & 1 & 4692.7 & -5.846 & $4.32 \times 10^{2}$ \\
\hline $5 p^{5} 7 g$ & ${ }^{1} H^{o}$ & 5 & $5 p^{5} 7 g$ & ${ }^{3} H^{o}$ & 4 & 4687.57 & -5.506 & $9.46 \times 10^{2}$ \\
\hline $5 p^{5} 7 g$ & ${ }^{1} G^{o}$ & 4 & $5 p^{5} 7 g$ & ${ }^{3} H^{o}$ & 4 & 4742.83 & -5.823 & $4.46 \times 10^{2}$ \\
\hline $5 p^{5} 7 g$ & ${ }^{3} F^{o}$ & 3 & $5 p^{5} 7 g$ & ${ }^{3} F^{o}$ & 4 & 4669.45 & -5.753 & $5.41 \times 10^{2}$ \\
\hline $5 p^{5} 7 g$ & ${ }^{3} F^{o}$ & 4 & $5 p^{5} 7 g$ & ${ }^{3} F^{o}$ & 4 & 4711.88 & -5.864 & $4.11 \times 10^{2}$ \\
\hline $5 p^{5} 9 d$ & ${ }^{3} F^{o}$ & 4 & $5 p^{5} 7 g$ & ${ }^{3} F^{o}$ & 4 & 4736.87 & -6.31 & $1.46 \times 10^{2}$ \\
\hline $5 p^{5} 7 g$ & ${ }^{3} G^{o}$ & 5 & $5 p^{5} 7 g$ & ${ }^{3} F^{o}$ & 4 & 4741.53 & -5.89 & $3.83 \times 10^{2}$ \\
\hline $5 p^{5} 7 g$ & ${ }^{3} H^{o}$ & 6 & $5 p^{5} 7 g$ & ${ }^{3} H^{o}$ & 5 & 4678.59 & -5.43 & $1.13 \times 10^{3}$ \\
\hline $5 p^{5} 7 g$ & ${ }^{3} F^{o}$ & 4 & $5 p^{5} 7 g$ & ${ }^{3} H^{o}$ & 5 & 4711.33 & -6.316 & $1.45 \times 10^{2}$ \\
\hline $5 p^{5} 7 g$ & ${ }^{3} G^{o}$ & 5 & $5 p^{5} 7 g$ & ${ }^{3} H^{o}$ & 5 & 4740.97 & -5.726 & $5.58 \times 10^{2}$ \\
\hline $5 p^{5} 7 g$ & ${ }^{3} F^{o}$ & 2 & $5 p^{5} 7 g$ & ${ }^{3} G^{o}$ & 3 & 4651.81 & -5.86 & $4.25 \times 10^{2}$ \\
\hline $5 p^{5} 7 g$ & ${ }^{3} G^{o}$ & 3 & $5 p^{5} 7 g$ & ${ }^{3} G^{o}$ & 3 & 4704.2 & -6.121 & $2.28 \times 10^{2}$ \\
\hline $5 p^{5} 7 g$ & ${ }^{1} G^{o}$ & 4 & $5 p^{5} 7 g$ & ${ }^{3} G^{o}$ & 3 & 4731.78 & -5.996 & $3.01 \times 10^{2}$ \\
\hline $5 p^{5} 9 d$ & ${ }^{3} F^{o}$ & 3 & $5 p^{5} 7 g$ & ${ }^{3} G^{o}$ & 3 & 4739.01 & -6.315 & $1.44 \times 10^{2}$ \\
\hline $5 p^{5} 7 g$ & ${ }^{3} G^{o}$ & 3 & $5 p^{5} 9 d$ & ${ }^{3} F^{o}$ & 2 & 4693.37 & -6.091 & $2.46 \times 10^{2}$ \\
\hline $5 p^{5} 9 d$ & ${ }^{3} F^{o}$ & 3 & $5 p^{5} 9 d$ & ${ }^{3} F^{o}$ & 2 & 4728.03 & -5.923 & $3.57 \times 10^{2}$ \\
\hline $5 p^{5} 9 d$ & ${ }^{1} D^{o}$ & 2 & $5 p^{5} 9 d$ & ${ }^{3} F^{o}$ & 2 & 4758.94 & -6.315 & $1.43 \times 10^{2}$ \\
\hline $5 p^{5} 9 d$ & ${ }^{3} P^{o}$ & 2 & $5 p^{5} 9 d$ & ${ }^{3} P^{o}$ & 2 & 4701.34 & -6.132 & $2.23 \times 10^{2}$ \\
\hline $5 p^{5} 9 d$ & ${ }^{1} D^{o}$ & 2 & $5 p^{5} 9 d$ & ${ }^{3} P^{o}$ & 2 & 4735.97 & -6.424 & $1.12 \times 10^{2}$ \\
\hline $5 p^{5} 9 d$ & ${ }^{3} D^{o}$ & 3 & $5 p^{5} 9 d$ & ${ }^{3} P^{o}$ & 2 & 4761.25 & -6.057 & $2.58 \times 10^{2}$ \\
\hline $5 p^{5} 9 d$ & ${ }^{1} P^{o}$ & 1 & $5 p^{5} 9 d$ & ${ }^{3} P^{o}$ & 2 & 4900.82 & -6.166 & $1.90 \times 10^{2}$ \\
\hline $5 p^{5} 7 g$ & ${ }^{3} F^{o}$ & 4 & $5 p^{5} 9 d$ & ${ }^{3} F^{o}$ & 3 & 4656.63 & -6.21 & $1.90 \times 10^{2}$ \\
\hline $5 p^{5} 9 d$ & ${ }^{3} F^{o}$ & 4 & $5 p^{5} 9 d$ & ${ }^{3} F^{o}$ & 3 & 4681.03 & -5.721 & $5.79 \times 10^{2}$ \\
\hline $5 p^{5} 9 d$ & ${ }^{3} D^{o}$ & 3 & $5 p^{5} 9 d$ & ${ }^{3} F^{o}$ & 3 & 4746.49 & -5.983 & $3.08 \times 10^{2}$ \\
\hline $5 p^{5} 7 i$ & ${ }^{1} K^{o}$ & 7 & $5 p^{5} 7 i$ & ${ }^{3} K^{o}$ & 6 & 4697.4 & -5.37 & $1.29 \times 10^{3}$ \\
\hline $5 p^{5} 7 i$ & ${ }^{3} I^{o}$ & 5 & $5 p^{5} 7 i$ & ${ }^{3} K^{o}$ & 6 & 4711.69 & -6.057 & $2.64 \times 10^{2}$ \\
\hline $5 p^{5} 7 i$ & ${ }^{1} I^{o}$ & 6 & $5 p^{5} 7 i$ & ${ }^{3} K^{o}$ & 6 & 4716.04 & -5.646 & $6.77 \times 10^{2}$ \\
\hline
\end{tabular}


TABLE VII (cont.)

\begin{tabular}{|c|c|c|c|c|c|c|c|c|}
\hline \multicolumn{3}{|c|}{ Lower level } & \multicolumn{3}{|c|}{ Upper level } & $\lambda[\AA]$ & $\log (g f)$ & $g A_{j i}\left[\mathrm{~s}^{-1}\right]$ \\
\hline Conf. & Term & $J$ & Conf. & Term & $\mathrm{J}$ & HFR & HFR & HFR \\
\hline $5 p^{5} 7 i$ & ${ }^{3} H^{o}$ & 4 & $5 p^{5} 7 i$ & ${ }^{3} I^{O}$ & 5 & 4693.21 & -5.588 & $7.82 \times 10^{2}$ \\
\hline $5 p^{5} 7 i$ & ${ }^{3} K^{o}$ & 8 & $5 p^{5} 7 i$ & ${ }^{3} K^{o}$ & 7 & 4697.4 & -5.311 & $1.48 \times 10^{3}$ \\
\hline $5 p^{5} 7 i$ & ${ }^{3} I^{o}$ & 5 & $5 p^{5} 7 i$ & ${ }^{3} I^{o}$ & 5 & 4711.56 & -5.65 & $6.73 \times 10^{2}$ \\
\hline $5 p^{5} 7 i$ & ${ }^{3} H^{o}$ & 6 & $5 p^{5} 7 i$ & ${ }^{3} K^{o}$ & 7 & 4711.68 & -5.982 & $3.14 \times 10^{2}$ \\
\hline $5 p^{5} 7 i$ & ${ }^{1} I^{o}$ & 6 & $5 p^{5} 7 i$ & ${ }^{3} I^{o}$ & 5 & 4715.9 & -5.847 & $4.26 \times 10^{2}$ \\
\hline $5 p^{5} 7 i$ & ${ }^{3} I^{o}$ & 7 & $5 p^{5} 7 i$ & ${ }^{3} K^{o}$ & 7 & 4716.03 & -5.579 & $7.91 \times 10^{2}$ \\
\hline $5 p^{5} 7 i$ & ${ }^{3} H^{o}$ & 5 & $5 p^{5} 7 i$ & ${ }^{3} H^{o}$ & 6 & 4693.24 & -5.508 & $9.39 \times 10^{2}$ \\
\hline $5 p^{5} 7 i$ & ${ }^{3} H^{o}$ & 6 & $5 p^{5} 7 i$ & ${ }^{3} H^{o}$ & 6 & 4711.56 & -5.58 & $7.91 \times 10^{2}$ \\
\hline $5 p^{5} 7 i$ & ${ }^{3} I^{o}$ & 7 & $5 p^{5} 7 i$ & ${ }^{3} H^{o}$ & 6 & 4715.91 & -5.784 & $4.93 \times 10^{2}$ \\
\hline $5 p^{5} 9 d$ & ${ }^{3} P^{o}$ & 1 & $5 p^{5} 9 d$ & ${ }^{3} D^{o}$ & 1 & 4571.83 & -6.248 & $1.80 \times 10^{2}$ \\
\hline $5 p^{5} 9 d$ & ${ }^{1} D^{o}$ & 2 & $5 p^{5} 9 d$ & ${ }^{3} D^{o}$ & 1 & 4647.9 & -6.343 & $1.40 \times 10^{2}$ \\
\hline $5 p^{5} 10 s$ & ${ }^{1} P^{o}$ & 1 & $5 p^{5} 10 s$ & ${ }^{3} P^{o}$ & 0 & 4731.72 & -6.255 & $1.66 \times 10^{2}$ \\
\hline $5 p^{5} 10 s$ & ${ }^{3} P^{o}$ & 2 & $5 p^{5} 10 s$ & ${ }^{3} P^{o}$ & 1 & 4697.64 & -5.844 & $4.33 \times 10^{2}$ \\
\hline $5 p^{5} 8 g$ & ${ }^{1} H^{o}$ & 5 & $5 p^{5} 8 g$ & ${ }^{3} H^{o}$ & 4 & 4695.28 & -5.508 & $9.38 \times 10^{2}$ \\
\hline $5 p^{5} 8 g$ & ${ }^{3} G^{o}$ & 3 & $5 p^{5} 8 g$ & ${ }^{3} H^{o}$ & 4 & 4729.87 & -6.415 & $1.15 \times 10^{2}$ \\
\hline $5 p^{5} 8 g$ & ${ }^{1} G^{o}$ & 4 & $5 p^{5} 8 g$ & ${ }^{3} H^{o}$ & 4 & 4739.23 & -5.842 & $4.27 \times 10^{2}$ \\
\hline $5 p^{5} 8 d$ & ${ }^{3} F^{o}$ & 3 & $5 p^{5} 8 g$ & ${ }^{3} F^{o}$ & 4 & 4612.92 & -6.483 & $1.03 \times 10^{2}$ \\
\hline $5 p^{5} 8 g$ & ${ }^{3} F^{o}$ & 3 & $5 p^{5} 8 g$ & ${ }^{3} F^{o}$ & 4 & 4699.6 & -5.848 & $4.28 \times 10^{2}$ \\
\hline $5 p^{5} 8 g$ & ${ }^{3} F^{o}$ & 4 & $5 p^{5} 8 g$ & ${ }^{3} F^{o}$ & 4 & 4727.77 & -5.866 & $4.07 \times 10^{2}$ \\
\hline $5 p^{5} 8 g$ & ${ }^{3} G^{o}$ & 5 & $5 p^{5} 8 g$ & ${ }^{3} F^{o}$ & 4 & 4738.03 & -5.907 & $3.68 \times 10^{2}$ \\
\hline $5 p^{5} 10 d$ & ${ }^{3} F^{o}$ & 4 & $5 p^{5} 8 g$ & ${ }^{3} F^{o}$ & 4 & 4739 & -6.466 & $1.02 \times 10^{2}$ \\
\hline $5 p^{5} 8 g$ & ${ }^{3} H^{o}$ & 6 & $5 p^{5} 8 g$ & ${ }^{3} H^{o}$ & 5 & 4689.02 & -5.429 & $1.13 \times 10^{3}$ \\
\hline $5 p^{5} 8 g$ & ${ }^{3} F^{o}$ & 4 & $5 p^{5} 8 g$ & ${ }^{3} H^{o}$ & 5 & 4726.86 & -6.318 & $1.43 \times 10^{2}$ \\
\hline $5 p^{5} 8 g$ & ${ }^{3} G^{o}$ & 5 & $5 p^{5} 8 g$ & ${ }^{3} H^{o}$ & 5 & 4737.11 & -5.747 & $5.33 \times 10^{2}$ \\
\hline $5 p^{5} 8 g$ & ${ }^{3} F^{o}$ & 2 & $5 p^{5} 8 g$ & ${ }^{3} G^{o}$ & 3 & 4676.41 & -5.875 & $4.07 \times 10^{2}$ \\
\hline $5 p^{5} 8 g$ & ${ }^{3} G^{o}$ & 3 & $5 p^{5} 8 g$ & ${ }^{3} G^{o}$ & 3 & 4721.04 & -5.98 & $3.14 \times 10^{2}$ \\
\hline $5 p^{5} 8 g$ & ${ }^{1} G^{o}$ & 4 & $5 p^{5} 8 g$ & ${ }^{3} G^{o}$ & 3 & 4730.37 & -6.013 & $2.90 \times 10^{2}$ \\
\hline $5 p^{5} 10 d$ & ${ }^{3} F^{o}$ & 3 & $5 p^{5} 10 d$ & ${ }^{3} F^{o}$ & 2 & 4728.01 & -5.855 & $4.16 \times 10^{2}$ \\
\hline $5 p^{5} 10 d$ & ${ }^{3} D^{o}$ & 2 & $5 p^{5} 10 d$ & ${ }^{3} F^{o}$ & 2 & 4738.14 & -6.398 & $1.19 \times 10^{2}$ \\
\hline $5 p^{5} 10 d$ & ${ }^{3} D^{o}$ & 2 & $5 p^{5} 10 d$ & ${ }^{3} P^{o}$ & 2 & 4722.03 & -6.418 & $1.14 \times 10^{2}$ \\
\hline $5 p^{5} 10 d$ & ${ }^{1} D^{o}$ & 2 & $5 p^{5} 10 d$ & ${ }^{3} P^{o}$ & 2 & 4733.41 & -6.198 & $1.89 \times 10^{2}$ \\
\hline $5 p^{5} 10 d$ & ${ }^{3} D^{o}$ & 3 & $5 p^{5} 10 d$ & ${ }^{3} P^{o}$ & 2 & 4746.44 & -6.054 & $2.62 \times 10^{2}$ \\
\hline $5 p^{5} 7 d$ & ${ }^{1} P^{o}$ & 1 & $5 p^{5} 10 d$ & ${ }^{3} P^{o}$ & 2 & 4924.89 & -6.248 & $1.56 \times 10^{2}$ \\
\hline $5 p^{5} 8 g$ & ${ }^{3} F^{o}$ & 4 & $5 p^{5} 10 d$ & ${ }^{3} F^{o}$ & 3 & 4677.71 & -6.367 & $1.31 \times 10^{2}$ \\
\hline $5 p^{5} 10 d$ & ${ }^{3} F^{o}$ & 4 & $5 p^{5} 10 d$ & ${ }^{3} F^{o}$ & 3 & 4688.71 & -5.677 & $6.38 \times 10^{2}$ \\
\hline $5 p^{5} 10 d$ & ${ }^{3} D^{o}$ & 3 & $5 p^{5} 10 d$ & ${ }^{3} F^{o}$ & 3 & 4736.52 & -5.968 & $3.20 \times 10^{2}$ \\
\hline $5 p^{5} 8 i$ & ${ }^{1} K^{o}$ & 7 & $5 p^{5} 8 i$ & ${ }^{3} K^{o}$ & 6 & 4699.89 & -5.37 & $1.29 \times 10^{3}$ \\
\hline $5 p^{5} 8 i$ & ${ }^{3} I^{o}$ & 5 & $5 p^{5} 8 i$ & ${ }^{3} K^{o}$ & 6 & 4709.5 & -6.058 & $2.63 \times 10^{2}$ \\
\hline $5 p^{5} 8 i$ & ${ }^{1} I^{o}$ & 6 & $5 p^{5} 8 i$ & ${ }^{3} K^{o}$ & 6 & 4712.41 & -5.648 & $6.76 \times 10^{2}$ \\
\hline $5 p^{5} 8 i$ & ${ }^{3} H^{o}$ & 4 & $5 p^{5} 8 i$ & ${ }^{3} I^{o}$ & 5 & 4697.11 & -5.588 & $7.81 \times 10^{2}$ \\
\hline $5 p^{5} 8 i$ & ${ }^{3} I^{o}$ & 5 & $5 p^{5} 8 i$ & ${ }^{3} I^{o}$ & 5 & 4709.4 & -5.65 & $6.73 \times 10^{2}$ \\
\hline $5 p^{5} 8 i$ & ${ }^{1} I^{o}$ & 6 & $5 p^{5} 8 i$ & ${ }^{3} I^{o}$ & 5 & 4712.32 & -5.848 & $4.26 \times 10^{2}$ \\
\hline $5 p^{5} 8 i$ & ${ }^{3} K^{o}$ & 8 & $5 p^{5} 8 i$ & ${ }^{3} K^{o}$ & 7 & 4699.88 & -5.311 & $1.48 \times 10^{3}$ \\
\hline $5 p^{5} 8 i$ & ${ }^{3} H^{o}$ & 6 & $5 p^{5} 8 i$ & ${ }^{3} K^{o}$ & 7 & 4709.48 & -5.982 & $3.13 \times 10^{2}$ \\
\hline $5 p^{5} 8 i$ & ${ }^{3} I^{o}$ & 7 & $5 p^{5} 8 i$ & ${ }^{3} K^{o}$ & 7 & 4712.41 & -5.579 & $7.92 \times 10^{2}$ \\
\hline $5 p^{5} 8 i$ & ${ }^{3} H^{o}$ & 5 & $5 p^{5} 8 i$ & ${ }^{3} H^{o}$ & 6 & 4697.13 & -5.508 & $9.38 \times 10^{2}$ \\
\hline $5 p^{5} 8 i$ & ${ }^{3} H^{o}$ & 6 & $5 p^{5} 8 i$ & ${ }^{3} H^{o}$ & 6 & 4709.4 & -5.581 & $7.90 \times 10^{2}$ \\
\hline $5 p^{5} 8 i$ & ${ }^{3} I^{o}$ & 7 & $5 p^{5} 8 i$ & ${ }^{3} H^{o}$ & 6 & 4712.33 & -5.785 & $4.93 \times 10^{2}$ \\
\hline $5 p^{5} 10 d$ & ${ }^{3} P^{o}$ & 1 & $5 p^{5} 10 d$ & ${ }^{3} D^{o}$ & 1 & 4648.33 & -6.306 & $1.53 \times 10^{2}$ \\
\hline $5 p^{5} 10 d$ & ${ }^{3} D^{o}$ & 2 & $5 p^{5} 10 d$ & ${ }^{3} D^{o}$ & 1 & 4662.08 & -6.386 & $1.26 \times 10^{2}$ \\
\hline $5 p^{5} 9 g$ & ${ }^{1} H^{o}$ & 5 & $5 p^{5} 9 g$ & ${ }^{3} H^{o}$ & 4 & 4698.16 & -5.504 & $9.46 \times 10^{2}$ \\
\hline $5 p^{5} 9 g$ & ${ }^{3} G^{o}$ & 3 & $5 p^{5} 9 g$ & ${ }^{3} H^{o}$ & 4 & 4719.69 & -6.307 & $1.48 \times 10^{2}$ \\
\hline
\end{tabular}


TABLE VII (cont.)

\begin{tabular}{|c|c|c|c|c|c|c|c|c|}
\hline \multicolumn{3}{|c|}{ Lower level } & \multicolumn{3}{|c|}{ Upper level } & \multirow{2}{*}{$\begin{array}{l}\lambda[\AA] \\
\mathrm{HFR} \\
\end{array}$} & \multirow{2}{*}{$\begin{array}{c}\log (g f) \\
\text { HFR }\end{array}$} & \multirow{2}{*}{$\frac{g A_{j i}\left[\mathrm{~s}^{-1}\right]}{\mathrm{HFR}}$} \\
\hline Conf. & Term & $J$ & Conf. & Term & $\mathrm{J}$ & & & \\
\hline $5 p^{5} 9 g$ & ${ }^{1} G^{o}$ & 4 & $5 p^{5} 9 g$ & ${ }^{3} H^{o}$ & 4 & 4724.16 & -5.824 & $4.48 \times 10^{2}$ \\
\hline $5 p^{5} 9 g$ & ${ }^{3} F^{o}$ & 3 & $5 p^{5} 9 g$ & ${ }^{3} F^{o}$ & 4 & 4691.71 & -5.765 & $5.21 \times 10^{2}$ \\
\hline $5 p^{5} 9 g$ & ${ }^{3} F^{o}$ & 4 & $5 p^{5} 9 g$ & ${ }^{3} F^{o}$ & 4 & 4713.39 & -5.73 & $5.59 \times 10^{2}$ \\
\hline $5 p^{5} 9 g$ & ${ }^{3} G^{o}$ & 5 & $5 p^{5} 9 g$ & ${ }^{3} F^{o}$ & 4 & 4724.1 & -5.891 & $3.85 \times 10^{2}$ \\
\hline $5 p^{5} 9 g$ & ${ }^{3} H^{o}$ & 6 & $5 p^{5} 9 g$ & ${ }^{3} H^{o}$ & 5 & 4693.21 & -5.428 & $1.13 \times 10^{3}$ \\
\hline $5 p^{5} 9 g$ & ${ }^{3} F^{o}$ & 4 & $5 p^{5} 9 g$ & ${ }^{3} H^{o}$ & 5 & 4712.35 & -6.183 & $1.97 \times 10^{2}$ \\
\hline $5 p^{5} 9 g$ & ${ }^{3} G^{o}$ & 5 & $5 p^{5} 9 g$ & ${ }^{3} H^{o}$ & 5 & 4723.06 & -5.726 & $5.62 \times 10^{2}$ \\
\hline $5 p^{5} 9 g$ & ${ }^{3} F^{o}$ & 2 & $5 p^{5} 9 g$ & ${ }^{3} G^{o}$ & 3 & 4680.49 & -5.845 & $4.35 \times 10^{2}$ \\
\hline $5 p^{5} 9 g$ & ${ }^{3} G^{o}$ & 3 & $5 p^{5} 9 g$ & ${ }^{3} G^{o}$ & 3 & 4713.33 & -5.903 & $3.76 \times 10^{2}$ \\
\hline $5 p^{5} 9 g$ & ${ }^{1} G^{o}$ & 4 & $5 p^{5} 9 g$ & ${ }^{3} G^{o}$ & 3 & 4717.79 & -5.989 & $3.08 \times 10^{2}$ \\
\hline $5 p^{5} 9 i$ & ${ }^{1} K^{o}$ & 7 & $5 p^{5} 9 i$ & ${ }^{3} K^{o}$ & 6 & 4701.4 & -5.37 & $1.29 \times 10^{3}$ \\
\hline $5 p^{5} 9 i$ & ${ }^{3} I^{o}$ & 5 & $5 p^{5} 9 i$ & ${ }^{3} K^{o}$ & 6 & 4708.15 & -6.06 & $2.62 \times 10^{2}$ \\
\hline $5 p^{5} 9 i$ & ${ }^{1} I^{o}$ & 6 & $5 p^{5} 9 i$ & ${ }^{3} K^{o}$ & 6 & 4710.22 & -5.649 & $6.75 \times 10^{2}$ \\
\hline $5 p^{5} 9 i$ & ${ }^{3} H^{o}$ & 4 & $5 p^{5} 9 i$ & ${ }^{3} I^{o}$ & 5 & 4699.44 & -5.587 & $7.81 \times 10^{2}$ \\
\hline $5 p^{5} 9 i$ & ${ }^{3} I^{o}$ & 5 & $5 p^{5} 9 i$ & ${ }^{3} I^{o}$ & 5 & 4708.09 & -5.65 & $6.73 \times 10^{2}$ \\
\hline $5 p^{5} 9 i$ & ${ }^{1} I^{o}$ & 6 & $5 p^{5} 9 i$ & ${ }^{3} I^{o}$ & 5 & 4710.16 & -5.849 & $4.26 \times 10^{2}$ \\
\hline $5 p^{5} 9 i$ & ${ }^{3} K^{o}$ & 8 & $5 p^{5} 9 i$ & ${ }^{3} K^{o}$ & 7 & 4701.39 & -5.311 & $1.48 \times 10^{3}$ \\
\hline $5 p^{5} 9 i$ & ${ }^{3} H^{o}$ & 6 & $5 p^{5} 9 i$ & ${ }^{3} K^{o}$ & 7 & 4708.14 & -5.983 & $3.13 \times 10^{2}$ \\
\hline $5 p^{5} 9 i$ & ${ }^{3} I^{o}$ & 7 & $5 p^{5} 9 i$ & ${ }^{3} K^{o}$ & 7 & 4710.21 & -5.579 & $7.92 \times 10^{2}$ \\
\hline $5 p^{5} 9 i$ & ${ }^{3} H^{o}$ & 5 & $5 p^{5} 9 i$ & ${ }^{3} H^{o}$ & 6 & 4699.45 & -5.508 & $9.37 \times 10^{2}$ \\
\hline $5 p^{5} 9 i$ & ${ }^{3} H^{o}$ & 6 & $5 p^{5} 9 i$ & ${ }^{3} H^{o}$ & 6 & 4708.08 & -5.582 & $7.88 \times 10^{2}$ \\
\hline $5 p^{5} 9 i$ & ${ }^{3} I^{o}$ & 7 & $5 p^{5} 9 i$ & ${ }^{3} H^{o}$ & 6 & 4710.16 & -5.786 & $4.92 \times 10^{2}$ \\
\hline $5 p^{5} 10 g$ & ${ }^{1} H^{o}$ & 5 & $5 p^{5} 10 g$ & ${ }^{3} H^{o}$ & 4 & 4700.21 & -5.503 & $9.47 \times 10^{2}$ \\
\hline $5 p^{5} 10 g$ & ${ }^{3} G^{o}$ & 3 & $5 p^{5} 10 g$ & ${ }^{3} H^{o}$ & 4 & 4715.29 & -6.313 & $1.46 \times 10^{2}$ \\
\hline $5 p^{5} 10 g$ & ${ }^{1} G^{o}$ & 4 & $5 p^{5} 10 g$ & ${ }^{3} H^{o}$ & 4 & 4718.54 & -5.825 & $4.48 \times 10^{2}$ \\
\hline $5 p^{5} 10 g$ & ${ }^{3} F^{o}$ & 3 & $5 p^{5} 10 g$ & ${ }^{3} F^{o}$ & 4 & 4695 & -5.769 & $5.15 \times 10^{2}$ \\
\hline $5 p^{5} 10 g$ & ${ }^{3} F^{o}$ & 4 & $5 p^{5} 10 g$ & ${ }^{3} F^{o}$ & 4 & 4710.12 & -5.731 & $5.58 \times 10^{2}$ \\
\hline $5 p^{5} 10 g$ & ${ }^{3} G^{o}$ & 5 & $5 p^{5} 10 g$ & ${ }^{3} F^{o}$ & 4 & 4718.69 & -5.892 & $3.84 \times 10^{2}$ \\
\hline $5 p^{5} 10 g$ & ${ }^{3} H^{o}$ & 6 & $5 p^{5} 10 g$ & ${ }^{3} H^{o}$ & 5 & 4696.45 & -5.428 & $1.13 \times 10^{3}$ \\
\hline $5 p^{5} 10 g$ & ${ }^{3} F^{o}$ & 4 & $5 p^{5} 10 g$ & ${ }^{3} H^{o}$ & 5 & 4709.24 & -6.184 & $1.97 \times 10^{2}$ \\
\hline $5 p^{5} 10 g$ & ${ }^{3} G^{o}$ & 5 & $5 p^{5} 10 g$ & ${ }^{3} H^{o}$ & 5 & 4717.8 & -5.726 & $5.63 \times 10^{2}$ \\
\hline $5 p^{5} 10 g$ & ${ }^{3} F^{o}$ & 2 & $5 p^{5} 10 g$ & ${ }^{3} G^{o}$ & 3 & 4687.01 & -5.844 & $4.35 \times 10^{2}$ \\
\hline $5 p^{5} 10 g$ & ${ }^{3} G^{o}$ & 3 & $5 p^{5} 10 g$ & ${ }^{3} G^{o}$ & 3 & 4710.52 & -5.908 & $3.72 \times 10^{2}$ \\
\hline $5 p^{5} 10 g$ & ${ }^{1} G^{o}$ & 4 & $5 p^{5} 10 g$ & ${ }^{3} G^{o}$ & 3 & 4713.76 & -5.989 & $3.08 \times 10^{2}$ \\
\hline $5 p^{5} 10 i$ & ${ }^{1} K^{o}$ & 7 & $5 p^{5} 10 i$ & ${ }^{3} K^{o}$ & 6 & 4702.36 & -5.369 & $1.29 \times 10^{3}$ \\
\hline $5 p^{5} 10 i$ & ${ }^{3} I^{o}$ & 5 & $5 p^{5} 10 i$ & ${ }^{3} K^{o}$ & 6 & 4707.28 & -6.049 & $2.69 \times 10^{2}$ \\
\hline $5 p^{5} 10 i$ & ${ }^{1} I^{o}$ & 6 & $5 p^{5} 10 i$ & ${ }^{3} K^{o}$ & 6 & 4708.8 & -5.642 & $6.86 \times 10^{2}$ \\
\hline $5 p^{5} 10 i$ & ${ }^{3} K^{o}$ & 8 & $5 p^{5} 10 i$ & ${ }^{3} K^{o}$ & 7 & 4702.36 & -5.311 & $1.48 \times 10^{3}$ \\
\hline $5 p^{5} 10 i$ & ${ }^{3} H^{o}$ & 6 & $5 p^{5} 10 i$ & ${ }^{3} K^{o}$ & 7 & 4707.28 & -5.981 & $3.14 \times 10^{2}$ \\
\hline $5 p^{5} 10 i$ & ${ }^{3} I^{o}$ & 7 & $5 p^{5} 10 i$ & ${ }^{3} K^{o}$ & 7 & 4708.8 & -5.579 & $7.92 \times 10^{2}$ \\
\hline $5 p^{5} 10 i$ & ${ }^{3} H^{o}$ & 5 & $5 p^{5} 10 i$ & ${ }^{3} H^{o}$ & 6 & 4700.96 & -5.507 & $9.40 \times 10^{2}$ \\
\hline $5 p^{5} 10 i$ & ${ }^{3} H^{o}$ & 4 & $5 p^{5} 10 i$ & ${ }^{3} I^{o}$ & 5 & 4700.95 & -5.587 & $7.81 \times 10^{2}$ \\
\hline $5 p^{5} 10 i$ & ${ }^{3} H^{o}$ & 6 & $5 p^{5} 10 i$ & ${ }^{3} H^{o}$ & 6 & 4707.23 & -5.576 & $7.99 \times 10^{2}$ \\
\hline $5 p^{5} 10 i$ & ${ }^{3} I^{o}$ & 5 & $5 p^{5} 10 i$ & ${ }^{3} I^{o}$ & 5 & 4707.23 & -5.65 & $6.74 \times 10^{2}$ \\
\hline $5 p^{5} 10 i$ & ${ }^{3} I^{o}$ & 7 & $5 p^{5} 10 i$ & ${ }^{3} H^{o}$ & 6 & 4708.76 & -5.779 & $5.00 \times 10^{2}$ \\
\hline $5 p^{5} 10 i$ & ${ }^{1} I^{o}$ & 6 & $5 p^{5} 10 i$ & ${ }^{3} I^{o}$ & 5 & 4708.75 & -5.847 & $4.28 \times 10^{2}$ \\
\hline
\end{tabular}




\section{References}

[1] G.L. Epstein, J. Reader, J. Opt. Soc. Am. 69, 511 (1979).

[2] E. Eliav, U. Kaldor, Chem. Phys. 392, 78 (2012).

[3] B. Karaçoban Usta, E. Sirin Yıldırım, AIP Conf. Proc. 2042, 020017 (2018).

[4] E. Biémont, M. Clar, S.Y. Enzonga, V. Fivet, P. Quinet, E. Träbert, H.P. Garnir, Can. J. Phys. 87, 1275 (2009).
[5] A.V. Loginov, Opt. Spectrosc. 122, 345 (2017).

[6] A. Kramida, Yu. Ralchenko, J. Reader, and NIST ASD Team, NIST Atomic Spectra Database (version 5.7.1), National Institute of Standards and Technology, Gaithersburg (MD) 2019.

[7] B. Karaçoban Usta, E. Şirin Yıldırım, AIP Conf. Proc. 2042, 020018 (2018). 